\title{
AN INVESTIGATION OF DAMAGE ARRESTMENT DEVICES ON CARBON FIBER SANDWICH SPECIMENS UNDER DYNAMIC LOADING
}

\author{
A Thesis \\ Presented to the Faculty of \\ California Polytechnic State University, San Luis Obispo
}

In Partial Fulfillment of the Requirements

for the Degree of Master of Science

in Aerospace Engineering

by

Gabriel Sabino Sanchez

June 2012 
(C) 2008

Gabriel Sabino Sanchez ALL RIGHTS RESERVED 


\section{COMMITTEE MEMBERSHIP}

TITLE: An Investigation of Damage Arrestment Devices on Carbon Fiber Sandwich Specimen under Dynamic Loading

AUTHOR: Gabriel Sabino Sanchez

DATE SUBMITTED: June 2012

Dr. Faysal Kolkailah

Committee Chair

Dr. Eltahry Elghandour

Committee Member

Dr. Eric Kasper

Committee Member

Dr. Susan Opava

Committee Member
Signature

Signature

Signature

Signature 


\begin{abstract}
An Investigation of Damage Arrestment Devices on Carbon Fiber Sandwich Specimen under Dynamic Loading
\end{abstract}

By:

\title{
Gabriel Sanchez
}

Debonding is one of the most severe defects associated with sandwich composite structures. The damage inflicted by debonding may lead to the significant degradation of the load carrying capacity of sandwich structures and affects their mechanical behaviors. In terms of dynamic loading, delamination could cause the natural frequency to decrease and potentially cause resonance to occur in the structure sooner than expected. This research studies the use of damage arrestment devices which are embedded between a carbon fiber facesheet and the foam core to find whether there is an increase in the structural integrity of the sandwich composites. Experimental and numerical finite element analyses are implemented for two different composite sandwich geometries: plates and beams. For each geometry set, delamination was intentionally placed between the foam and facesheet in different locations. The different placements of the delamination reside horizontally on the top, bottom, and middle of the composite beam and plate. However, for the plate case there are two vertical delamination cases that were placed in the middle and on the side of the composite sandwich plate. The delaminated composite specimens consisted of one-inch delamination regions running horizontally across the beam and plate, while the control specimens were without delamination between the carbon fiber facesheets and the foam core. When incorporating the damage arrestment devices known as shear keys inside the structure, two shear keys were placed along the edge of the delamination region. For these samples, the control shear key case did not have a delamination region while the second case incorporated both the shear keys and delamination. Each structure consisted of the same loading criteria and was restricted to the same vibration fixture during the experiment.

The location of the accelerometer placement was also considered for this research. The mass of the accelerometer would alter the frequency response of the specimen depending on the location of the accelerometer. Therefore, three locations were considered in order to study the effects of the accelerometer's location in terms of frequency response. These locations included the accelerometer placed directly in the center of the composite sandwich plate, three inches above the center of the plate, and finally 2.5 inches to the right of the center and two inches above. This procedure was done for all the composite sandwich plate cases. In order to compare numerical and experimental data, only the results 
from the accelerometer placed directly in the center of the composite plate were acquired to further study the frequency response of the composite sandwich. For the composite beam case, only the results of when the accelerometer was placed one inch from the top on the composite beam were examined when comparing numerical with experimental results.

The final case study that was conducted during this research was determining the effects of applying a vertical shear key inside the composite sandwich beam. Currently only shear keys placed horizontally across the composite sandwich beam were studied. For this case study a vertical shear key was placed in the center of the beam. Different delamination regions were then applied to the beam structure to determine the effects of having an increasing delamination region in terms of dynamic response. These delamination regions consisted of 1 x 1 inch, 2 x 1 inch, and 3 x 1inch delamination region. The frequency responses as well as the time responses were studied for all the different composite cases throughout the experiment.

The mechanical properties of LTM45/CF1803 pre-impregnated carbon fiber and Last-A-foam FR 6710 polyvinylchloride foam were experimentally analyzed using ASTM D3039 and ASTM D1621 standards respectively to determine the material's mechanical properties. By using the finite element program COSMOS with the pre-software GeoStar, in order to compare numerical and theoretical results. The experimental values and the numerical values were compared graphically and numerically through data assimilation. 


\section{ACKNOWLEDGEMENTS}

I want to first thank my thesis advisors, Dr. Faysal Kolkailah, and Dr. Eltahry Elghandour for providing me with opportunity to increase my knowledge on composite materials and stress analysis. I am grateful for their support, advice, and assistance at all stages of this project. In addition, I would like to thank Dr. Eric Kasper and Dr. Susan Opava for accepting my request and for being involved with my thesis committee. I would also like to thank all my committee for taking off time from their busy schedules to edit my thesis and assist me throughout this thesis experience. I would also like to acknowledge the Department of the Navy, Office of Naval Research, under Award \# N00014-08-1-1209, who sponsored this work.

I would like to give thanks to all my friends who supported and believed in me from the beginning of my college career. With their motivation and encouragement they have helped me through the toughest situations in my college and celebrated my achievements as well. I will always be indebted to them for their friendship and care, especially Ramon Sanchez and Justin Pryor.

I would also like to give thanks to my family. Without their love, guidance, and support, I would not be anywhere near where I am today. My family has always motivated me to pursue my goal and to strive to be the best I can. This thesis will not only be a testament to my hard work, but also for all my family members. Each family member has made sacrifices to provide me with a better opportunity to succeed. Also, thanks to my beloved Alma for her unconditional love, support, and encouragement throughout my college years.

I would also like to thank the Cal Poly Aerospace Engineering Department for accepting me to the graduate program. In addition, I would also like to thank Cody Thompson the lab technician, Denis Boucher, and Kodi Rider for their help in the lab and assistance on my thesis. Finally, I most importantly thank God for giving me the opportunity to pursue and fulfill my professional career. Without God in my life, I would have never gotten this far in life and would have not been the person that I am today. 


\section{TABLE OF CONTENTS}

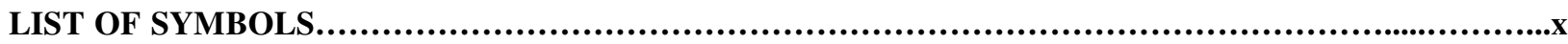

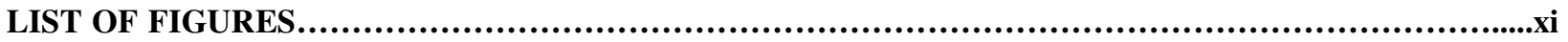

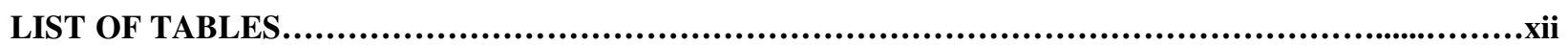

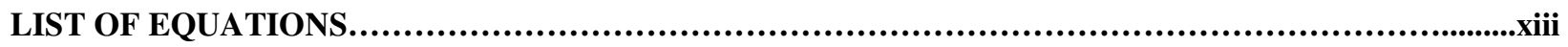

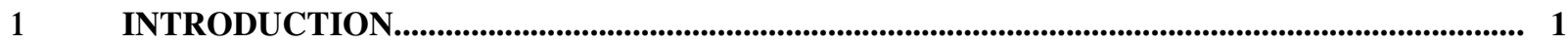

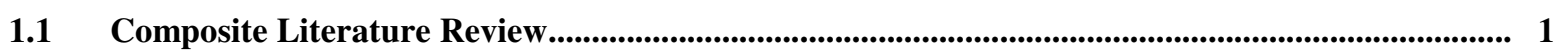

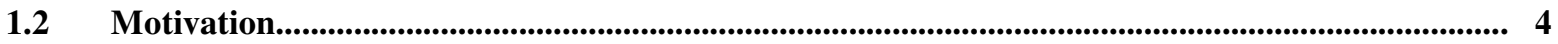

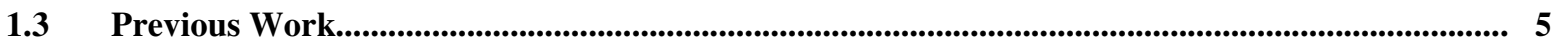

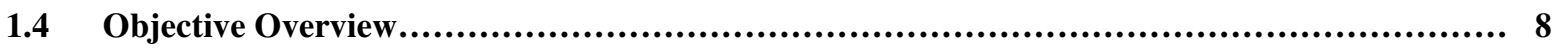

2 Manufacturing Procedures and Experimental Setup........................................................ 10

2.1 Mechanical Characteristics of Carbon Fiber Facesheets............................................................................ 10

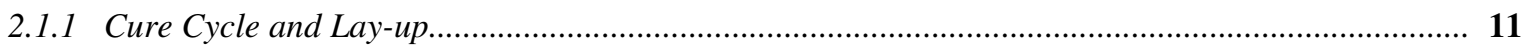

2.1.2 Carbon Fiber Facesheet Fabrication Methods.......................................................... 12

2.1.3 Elastic Modulus and Shear Modulus of the Carbon..................................... 15

2.14 Poisson's Ratio for the Carbon Fiber Facesheet................................................. 17

2.1.5 Density and Burn Test of the Carbon Fiber Facesheet..................................................... 19

2.2 Mechanical Characteristics of the Polyvinylchloride Foam................................................................. 20

2.2.1 LAST-A-Foam Cutting Methods.......................................................................... 21

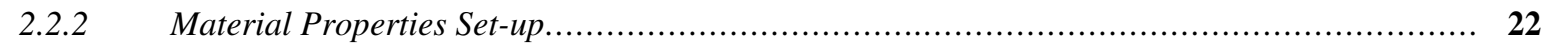

2.3 Mechanical Characteristics of Fiber Glass Shear Key........................................................................ 23

2.3.1 Fiber Glass Shear Keys Fabrication Method........................................................................... 23

2.3.2 Elastic Modulus and Shear Modulus of the Fiber Glass Shear Keys........................................... 24

2.3.3 Poisson's Ratio for the Fiberglass Shear Keys....................................................................... 25 
2.3.4 Density and Burn Test of the Fiber Glass Shear Keys

Composite Sandwich Plate Fabrication................................................................................. 27

2.4.1 Cure Cycle and Lay-up of the Composite Sandwich Plate......................................................... 27

2.4.2 Fabrication of the Control and Delamination Specimens.........................................................29

2.4.3 Fabrication of the Composite Sandwich Plate with Shear Keys............................................... 30

Composite Sandwich Beam Fabrication......................................................................................... 31

2.5.1 Construction of Composite Sandwich Beams....................................................................... 31

2.5.2 Fabrication of the Varying Delaminated Composite Sandwich Beams....................................... 32

3 DISCUSSION OF EXPERIMENTAL MATERIAL PROPERTIES........................................... 34

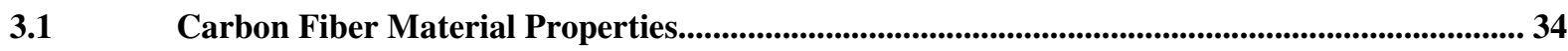

Polyvinylchloride Foam Material Properties.................................................................... 38

Fiberglass Material Properties.................................................................................................40

4 DYNAMIC RESPONSE OF THE COMPOSITE SANDWICH SPECIMENS....................... 44

Experimental Setup and Procedures......................................................................................44

Dynamic Response of the Composite Sandwich Plates.............................................................. 46

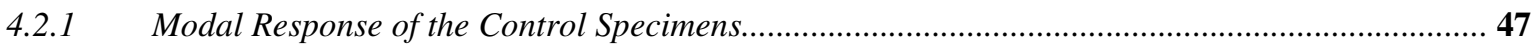

4.2.2 Modal Response of the Delaminated Specimens.....................................................................50

4.2.3 Modal Response of the Shear key-only Specimens.............................................................52

4.2.4 Modal Response of the Delamination Specimens with Shear Keys...........................................55

4.2.5 Comparison Between the Frequency Responses for Each Plate Configuration.............................. 58

Dynamic Response of the Composite Sandwich Beams............................................................ 63

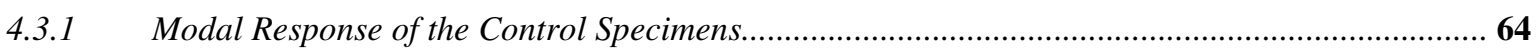

4.3.2 Modal Response of the Delaminated Specimens...................................................................65

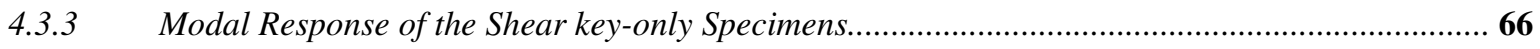

4.3.4 Modal Response of the Delamination Specimens with Shear Keys..............................................67

4.3.5 Comparison Between the Frequency Responses for Each Plate Configuration..............................68

Altering the Delamination Region for Composite Sandwich Beams........................................... 70 
4.5 Time Response for Various Composite Specimens.......................................................................... 73

4.6 Resonance Frequency to Weight Ratio................................................................................................ 76

$5 \quad$ NUMERICAL FINITE ELEMENT ANALYSIS......................................................................................... 78

5.1 Material Properties Selection for the Finite Element Analysis....................................................... 78

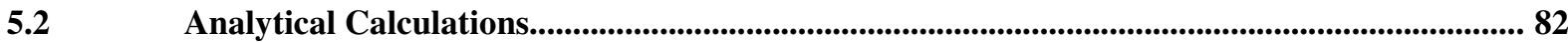

$5.3 \quad$ Sandwich Plate Finite Element Models.......................................................................................... 84

5.3.1 Constructing the Control Finite Element Model.......................................................................... 85

5.3.2 Constructing the Top Delaminated Finite Element Model Case ................................................... 88

5.3.3 Constructing the Bottom Shear Key Finite Element Model Case ................................................... 90

5.3.4 Constructing the Middle Delaminated Finite Element Model with Shear Keys Case...................... 93

S.4 Sandwich Beam Finite Element Models............................................................................................. 95

5.4.1 Constructing the Control Finite Element Model.......................................................................... 95

5.4.2 Constructing the Delaminated Finite Element Model Cases.......................................................... 98

5.4.3 Constructing the Shear Key Finite Element Model Cases.............................................................. 99

5.4.4 Constructing the Delaminated Finite Element Model with Shear Key Cases.................................101

5.4.5 Constructing the Vertical Shear Key Finite Element Model Cases.................................................102

6 COMPARISON BETWEEN NUMERICAL AND EXPERIMENTAL ANALYSIS...............................104

6.1 Graphical Representation of the Numerical and Experimental Results.......................................104

6.2 Error Percentage between the Numerical and Experimental Results.............................................108

7

CONCLUSTION_..........................................................................................................................................................110

7.1 Lessons Learned Throughout the Research...........................................................................11

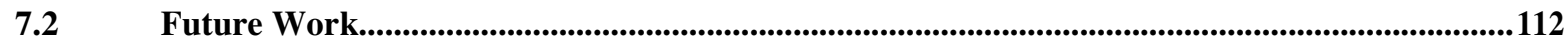

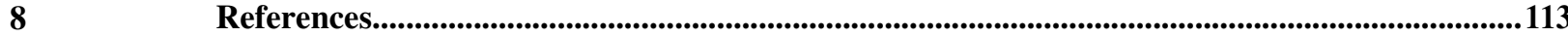

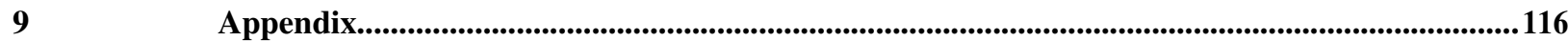




\section{LIST OF SYMBOLS}

\section{Symbol}

A

$\mathrm{A}_{\mathrm{ij}}$

$\mathrm{B}_{\mathrm{ij}}$

$\mathrm{D}_{\mathrm{ij}}$

E

F

G

$\mathrm{Hg}$

$\mathrm{Hz}$

I

M

$\mathrm{N}$

Mat

$\mathrm{Q}_{\mathrm{ij}}$

V

A

error

$\mathrm{H}$

j

S

$\mathrm{t}$

$\mathrm{u}(\mathrm{t})$

$\ddot{u}$

Z $\underline{\text { Description }}$

$\underline{\text { Unit }}$

Amplitude

g's

Extensional Stiffness

Bending Extensional Coupling Stiffness

Bending Stiffness

Young's Modulus of Elasticity

Fahrenheit

Shear Modulus

Mercury

Hertz

Rotary Inertias

Moment

Force

Material Property

Lamina Stiffness

Volume Fraction

Composite length

Average Error Percentage $\%$

Composite height

Amplitude Increment

Characteristic Root

Time

Amplitude of motion

Peak Acceleration

Vertical Lamina Coordinate psi

in.

in.

$1 / \mathrm{s}$

$\mathrm{s}$

g's

psi

psi

psi

psi

${ }^{\circ} \mathrm{F}$

psi

$1 / \mathrm{s}$

lbs. in ${ }^{2}$

lbs.in

lbs.

si

$\%$

g's

in. 


\section{Greek}

$\Omega$

$\varepsilon$

$\zeta$

$\rho$

$\sigma$

$\lambda$

v

$\omega_{\mathrm{n}}$

\section{$\underline{\text { Subscripts }}$}

$\exp$

$\mathrm{f}$

$\mathrm{m}$

ma

$\mathrm{t}$

$\mathrm{X}$
Ohm

Tensile Strain

Damping Ratio

Density

Normal Stress

Eigenvalue

Poisson's Ratio

Natural Frequency

$1 / \mathrm{s}$

psi
$\frac{J * S}{C}$

in/in

$\mathrm{lbs} / \mathrm{in}^{3}$

Experimental Properties

Fiber

Matrix

Manufacture's Properties

Total

Increment number 


\section{LIST OF FIGURES}

Figure 1: Various methods of creating a composite material. .............................................................. 2

Figure 2: Carbon fiber sandwich composite used for the experiment....................................................... 4

Figure 3: Jakobsen's peel-stopper design for stopping delamination in the face-core interface. ................. 6

Figure 4: Illustration of how actuators are mounted on a composite structure to alter natural frequency.... 7

Figure 5: LTM45/CF1803 layup provided by the Advance Composite Group....................................... 11

Figure 6: Curing cycle provided by the Advance Composite Group for the LTM45/CF1803 carbon fi....12

Figure 7: Material used to manufacture a carbon fiber sample.......................................................... 13

Figure 8: Prepreg carbon fiber role taken out of the Cal Poly Aerospace freezer. ................................... 14

Figure 9: American Autoclave located near Cal Poly Aerospace Laboratory. ......................................... 14

Figure 10: Pressure valves and vacuum pump located next to the American Autoclave. ......................... 15

Figure 11: 3M Scotch-Weld Epoxy Adhesive DP-460 used to attach aluminum tabs to specimens..........16

Figure 12: Tension test applied on a carbon fiber coupon using the Cal Poly Aerospace Instron...............17

Figure 13: Chemical components used to connect strain gages to fiberglass and carbon fiber test ...........18

Figure 14: Illustration of the connection needed in order to collect data from a Strain Gage. ..................19

Figure 15: Cal Poly Aerospace oven which is used in conducting burn test on composite samples..........20

Figure 16: Tile saw used to cut smaller Last-A-Foam pieces to conduct material property tests...............21

Figure 17: Compression test applied to the Last-A-Foam specimen to acquire the compressive................22

Figure 18: Aeropoxy hardener and resin used for the fiberglass shear keys........................................... 23

Figure 19: Distributed force applied onto the aluminum plate while the fiberglass shear keys cure. .........24

Figure 20: Illustration of strain gage attachment implement of the fiberglass coupon samples.................26

Figure 21: Vacuum pump connections to the complete sandwich composite lay-up..............................28

Figure 22: Tile saw used to cut all composite specimens and foam pieces. ..............................................29

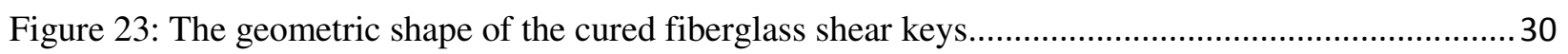

Figure 24: Different cases studies of the carbon fiber sandwich composite beams.................................. 32

Figure 25: Normal stress and normal strain curve of the carbon fiber facesheet..................................... 35

Figure 26: Fracture locations of the carbon fiber specimens after tensile testing..................................... 35

Figure 27: Transverse and longitudinal strain curve relationship of the carbon fiber specimens...............36

Figure 28: Stress and strain curve for both parallel and perpendicular to the rise of the Last-A-Foam. ....39

Figure 29: Normal Stress and Normal strain curves for both fiber directions of the fiberglass samples.... 40

Figure 30: Transverse and longitudinal strain curve relationship for both fiber directions of the .............42

Figure 31: Set-up for the composite sandwich specimens to undergo vibration test.................................45

Figure 32: Placement of the accelerometer for both the plate and beam experiments..............................46

Figure 33: Location of the shear key and/or delamination for the composite sandwich plates. .................47

Figure 34: Frequency response for the control specimen 1 for various accelerometer locations. .............. 48

Figure 35: Frequency response for all three control plate specimens for when the accelerometer is......... 49

Figure 36: Amplitude and frequency response for all control specimens............................................... 49

Figure 37: Frequency response for the middle delaminated specimen 1 for various accelerometer ..........50

Figure 38: Frequency response for all three middle delaminated plate specimens for when the ...............51 
Figure 39: Amplitude and frequency response for all middle delaminated specimens.............................51

Figure 40: Amplitude and frequency response for all bottom delaminated specimens. ...........................52

Figure 41: Frequency response for specimen 1 with only shear keys in the middle of the plate for .......... 53

Figure 42: Frequency response for all three middle shear keys only plate specimens for when the ..........54

Figure 43: Amplitude and frequency response for all middle shear keys only specimens. .......................54

Figure 44: Amplitude and frequency response for all top shear keys only specimens............................55

Figure 45: Frequency response for specimen 1 with the middle delamination plate with shear keys .........56

Figure 46: Frequency response for all three middle delamination with shear keys plate specimens for .... 56

Figure 47: Amplitude and frequency response for all middle delamination specimens with shear keys. ..57

Figure 48: Amplitude and frequency response for all side delamination specimens with shear keys in ....58

Figure 49: Average amplitude and frequency response for all cases that involve a middle delamination .60

Figure 50: Average amplitude and frequency response for all cases that involve a bottom delamination.61

Figure 51: Average amplitude and frequency response for all cases that involve a top delamination .......62

Figure 52: Average amplitude and frequency response for all cases that involve a side delamination......63

Figure 53: Average amplitude and frequency response for all cases that involve a middle......................63

Figure 54: Frequency responses for all composite sandwich control specimens...................................65

Figure 55: Frequency responses for all middle delaminated composite specimens. ................................66

Figure 56: Frequency responses for all middle shear keys only composite specimens...........................67

Figure 57: Frequency responses for all middle delaminated composite specimens with shear keys.........67

Figure 58: Comparison between the Average amplitude and frequency response for all cases that .........68

Figure 59: Comparison between the Average amplitude and frequency response for all cases ................69

Figure 60: Comparison between the Average amplitude and frequency response for all cases that .......... 70

Figure 61: The various delamination regions for the vertical shear key composite sandwich beam.......... 71

Figure 62: Frequency response for all first specimens for each case study......................................... 72

Figure 63: Average amplitude and frequency response for all composite sandwich beam cases with....... 73

Figure 64: Time response for the carbon fiber plate specimens for the bottom (right) and middle (left)... 75

Figure 65: Time response for the middle carbon fiber sandwich beam case......................................... 75

Figure 66: Time response for all the vertical shear cases with different delamination regions and ........... 76

Figure 67: Comparison between the carbon fiber numerical finite element values to the vibration table.. 79

Figure 68: Evaluating the experimental and manufacturing finite element models to the vibrated foam .. 80

Figure 69: Finite element model of the control plate using GeoStar and COSMOS. ...............................87

Figure 70: Finite element model of the top delaminated plate created on the pre-software GeoStar.........90

Figure 71: Representation of the finite element geometry for shear keys embedded into the foam...........91

Figure 72: Finite element models of the bottom delaminated case using GeoStar and COSMOS to.........92

Figure 73: Finite element models of the middle delaminated with shear keys case using GeoStar and.....94

Figure 74: Finite element models of the control beam with (right) and without (left) the accelerometer .. 97

Figure 75: Finite element results for the middle delaminated case study............................................99

Figure 76: Finite element results for the bottom shear key-only case study......................................... 100

Figure 77: Finite element models of the middle delaminated with shear keys case using GeoStar. ........101

Figure 78: Finite element models of the 2 inch delaminated without shear keys in the top row and ....... 103

Figure 79: Graphical representation between the experimental and finite element results for the ........... 105

Figure 80: Middle case study comparison between the numerical and experimental results. .................. 106 
Figure 81: Graphical representation of the finite element and experimental results from the middle...... 106 Figure 82: Graphical representation of the finite element and experimental results from the top beam .. 107 Figure 83: Depiction of the numerical and experimental results for the vertical shear key case for ........ 108 


\section{LIST OF TABLES}

Table 1: Burn test results of the $1 \times 1$ inch carbon fiber samples to acquire the weight fraction of ............37

Table 2: Mechanical characteristics for the two different carbon fiber lay-ups........................................ 38

Table 3: Comparison between the manufacture and experimental mechanical characteristics for............. 39

Table 4: Burn test results of the $1 \times 1$ inch fiberglass samples to acquire the weight fraction of the ......... 42

Table 5: Woven Roving Fiberglass with Aeropoxy resin composite material properties.......................... 43

Table 6: Comparison between the different accelerometer locations for each composite sandwich...........59

Table 7: Comparison between the weight and the resonance frequency for the middle case at the first....76

Table 8: Relationship between the numerical and experimental values for the carbon fiber plate.............79

Table 9: Manufacturer's, experimental, and average material properties for Last-A-Foam FR $6710 \ldots . . . . .81$

Table 10: Material properties implemented onto the finite element models........................................... 81

Table 11: Error percentage between the experimental and numerical results for the control sandwich..... 88

Table 12: Finite element results for the first four modes that were detected by the accelerometer for .......90

Table 13: Finite element results for the first four modes that were detected by the accelerometer for ...... 93

Table 14: The Finite element results of the first four modes that were detected by the accelerometer...... 95

Table 15: Error percentage between the finite element results with and without the accelerometer..........98

Table 16: Finite element results for the first four modes that were detected by the accelerometer for ...... 99

Table 17: Finite element results for the first two bending modes for each delaminated only case study. 100

Table 18: The Finite element results for the first two bending modes for each delamination with shear 102

Table 19: The Finite element results for the first two bending modes of the vertical shear key case .......103

Table 20: Shows the error percentage among the numerical and experimental results for several case ...109 


\section{LIST OF EQUATIONS}

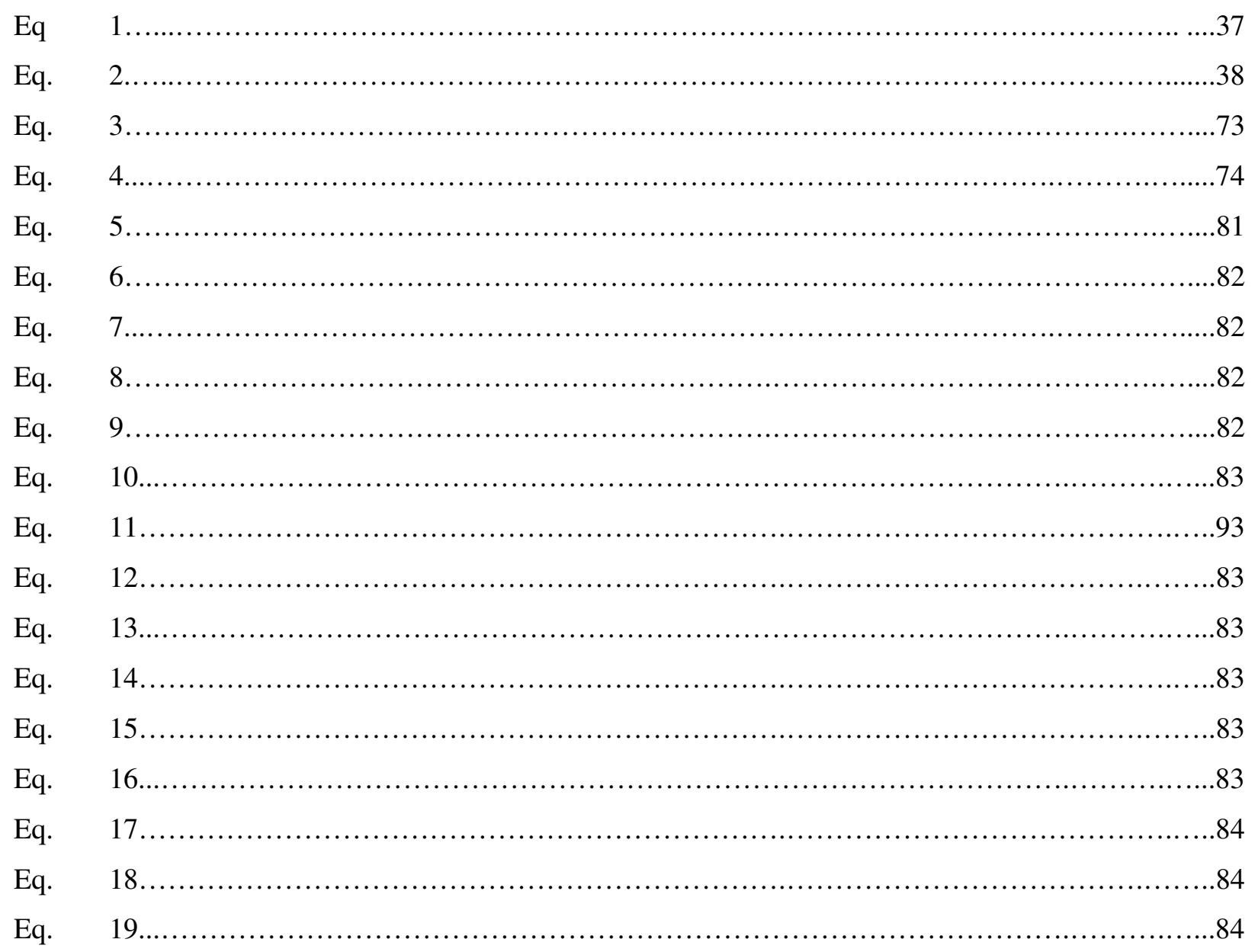




\section{INTRODUCTION}

\subsection{Literature Review}

Composites are primarily comprised of two or more materials and are a major aspect of the manufacturing engineering. Combining materials offer several advantages over a single material. Composites provide needed properties that the combined materials offer. Some designs could require high strength and high electrical conductivity. While alloys usually provide high strength and pure metals offer good conduction; composites can have both properties with the proper materials selected. Composites exhibit desirable physical and chemical properties that include lightweight properties with high stiffness and strength along the direction of the reinforcing fiber, dimensional stability, temperature and chemical resistance, and a wide range of performance. Composites are replacing metal components in many uses, particularly in the aerospace industry. A common type of composite is the fiber-matrix type. Fibers are the material that improves the stiffness of the composite, and the matrix is usually a resin that further holds the fibers together. Composites are made from embedding the fibers or particles in a continuous matrix of a polymer, metal, or ceramic. Composites have high stiffness and strength per unit weight and in some cases, such as a metal and a ceramic matrix composite, can have high temperature performance ${ }^{1}$. Fiber-reinforced composite materials can be divided into two main categories normally referred to as short fiber-reinforced materials and continuous fiber-reinforced materials. Chopped fiber structure and a particulate layout are common short fiber-reinforced composites. Continuous reinforced materials will

often constitute a layered or laminated structure. The woven and continuous fiber styles are typically available in a variety of forms, including being pre-impregnated with the given matrix, dry, unidirectional tapes of various widths, plain weave, harness satins, braided, or stitched ${ }^{2}$. Composite materials exist in nature almost everywhere and one of the best examples is wood. The grain seen in wood is strong in tension, while the supporting matrix of lignin is strong in compression. Wood tends to have different material properties with and against the grain, which is not only a characteristic of wood, but of most modern composites.

There are several methods in combining composite materials together such as a laminate, sandwich panel, unidirectional, chopped fiber, and particulates. Figure 1 shows the several types of combinations that a composite material can have. Sandwich panels provide bending stiffness at a relatively low weight. The lightweight core separates the skins without contributing directly to the bending resistance. One critical component to having a successful sandwich composite is having good quality adhesive joints between the skin and the core. Laminates offer another method of increasing the 
stiffness associated with the composite. Laminates can be used for both aerospace and civil engineering applications. The lamination process distributes the defects in the material, reduces property variability, and enables much larger plate structures to be built. However, there are disadvantages of using laminate for any application. Laminates tend to be very resin-rich and therefore are excessively heavy. Only short fibers are incorporated which severely limits the mechanical properties of the laminate. Also the resins need to be low in viscosity which generally compromises their mechanical and thermal properties.
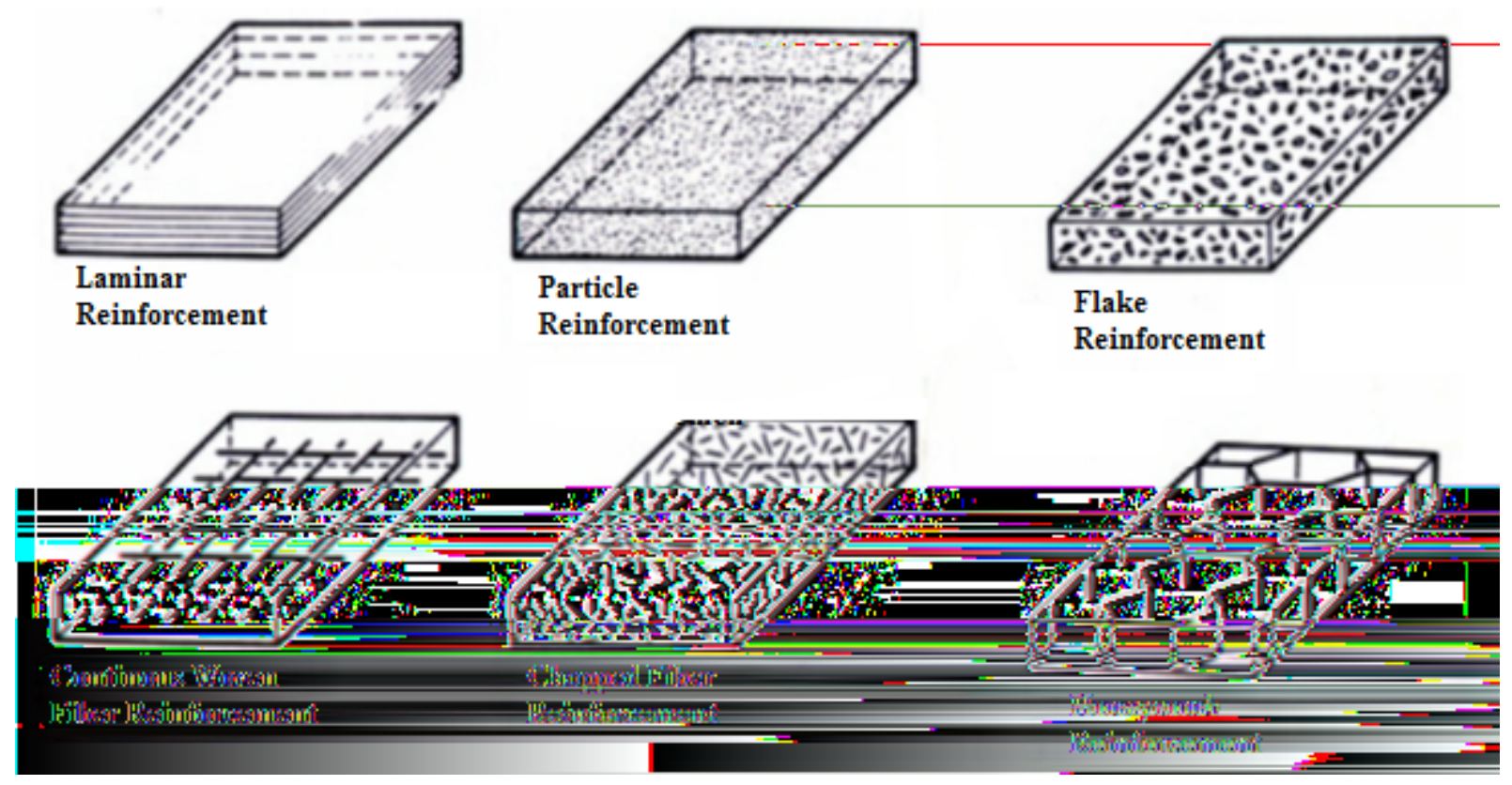

Figure 1: Various methods of creating a composite material.

Furthermore, it is pertinent to understand the concept of a composite laminate. A laminate is a bonded stack of lamina, where the fibers can be oriented symmetrically or anti-symmetrically about the middle surface of the laminate. The purpose of lamination is to tailor the directional dependence of strength and stiffness of a composite material to match the loading environment of the structural element. Laminates are suitable for this purpose because the principal material directions of each layer can be oriented according to each requirement. For example, to obtain an extensional stiffness far higher in one direction than the other, half of the layers in a laminate should be oriented in a ninety degree direction from the other half of the layers in the laminate. Symmetry about the middle surface of a laminate is often desirable to avoid coupling between bending and extension. In order to achieve particular design requirements, the use of unsymmetrical laminates is necessary. This is true especially for a form of coupling necessary to make jet turbine fan blades with pre-twist without the use of a complex mold. Antisymmetric laminates must have an even number of layers if the adjacent lamina also has altering signs of the principal material property directions with respect to the laminate axes. If the adjacent lamina does not 
have altering signs, this is not necessary and the number of layers does not have to be even. A common type of an antisymmetric laminate is a cross-ply antisymmetric laminate which consists of an even number of orthotropic lamina laid on each other with principal material directions alternating at zero degrees and ninety degrees to the laminate axes. A more complicated cross-ply laminate is one where the adjacent layers do not always have an alternating sequence of zero and ninety degrees. A regular antisymmetric angle ply laminate has all lamina with equal thicknesses and is common due to the simplicity of fabrication. As the number of layers increases, the coupling stiffness will approach zero. A less common type of an antisymmetric laminate is that of an angle-ply laminate. This type has lamina oriented at $+\alpha$ degrees to the laminate coordinate axes on one side of the middle surface and the corresponding equal-thickness lamina oriented at $-\alpha$ degrees on the other side at the same distance from the middle surface. A more complicated version will include mixed materials with mixed lamination angles. A regular antisymmetric angle-ply laminate has all the lamina of the same material and thickness for ease of fabrication. As the number of layers in the laminate increases the bending-extension coupling stiffness will approach zero. Overall, asymmetric laminates are the more general class of laminate, where lack of symmetry often occurs by design as in deliberately constructing a non-symmetric laminate. Further, often symmetric laminates subjected in service to heating from one side so that the resulting thermal gradient acting on the temperature dependent material properties renders the laminate unsymmetric. ${ }^{3}$

Laminated composite comprised of different materials that are bonded to each other is considered to be a sandwich structure. This composite utilize the properties of each separate component to the structural advantage of the whole assembly. A sandwich structure, as seen in Fig. 2, is one that is layered with two robust facesheets that are bonded to a lightweight and compliant core. Utilizing thin facesheets with high stiffness along with low density cores gives an overall specimen with a high stiffness-to-weight ratio and a high bending strength-to-weight ratio. Sandwich composites have the continuous support of the facesheet, unlike a stiffened structure, which implies that surfaces can remain flat even under quite high compressive stress without buckling. The ability of sandwich structures to remain flat under high loads is a very important quality that aircraft control surfaces must have. The use of foam cores in sandwich structures allows for them to be of great use in spacecraft because of the additional thermal insulation from the core; ensuring a low structural weight. However, foam composites do impose a possibility of outgassing during the life of the structure. 


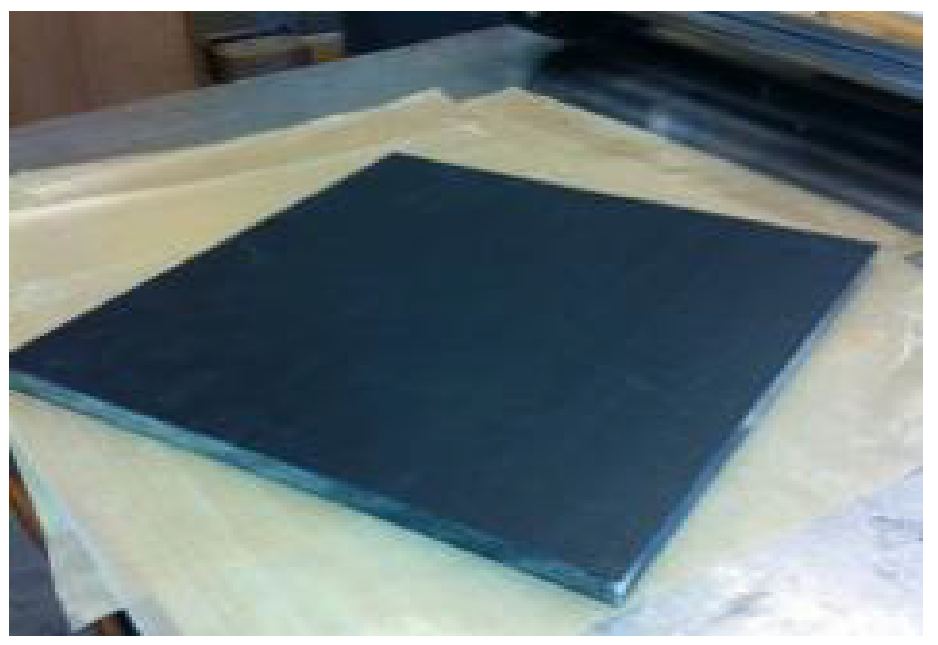

Figure 2: Carbon fiber sandwich composite used for the experiment.

Sandwich composites also are free from any nuts, bolts, or rivets ensuring a continuous design, which is a more structurally sound design method. Unlike metals, sandwich composites can be manufactured in one piece, thus reducing assembly costs and ensuring smooth and continuous load paths. As with most design methods, there are various disadvantages associated with the sandwich structure. Sandwich material composites are more prone to delamination between the core and facesheet. Being a relatively new material, compared to metals and plastic structures, there are not nearly as many standards for manufacturing methods. When there are no standards or specifications, any type of quality control is a difficult task especially when the structure being made is an aircraft or spacecraft that must pass the most strict design requirements. The limited data available on composites forces engineers to be extra cautious and conservative in their designs thus designing the structures heavier than needed, which contradicts the main goal of composites of saving weight.

\subsection{Motivation}

The mechanical characteristics of composites are a great value to some applications of civil infrastructure, and aerospace applications. Composites are extremely versatile because they can be designed and manufactured to the material properties specific to a given application. Additionally, composites are able to reduce total weight by significant amounts, which in turn allow applications such as aircraft to travel farther and faster. However, because composites require meticulous fabrication and quality control, the cost of composites is much higher than traditional materials. Furthermore, the cost of maintenance also is much higher for composites. However, many composite materials give rise to stable cracks growth before instability. Unlike metallic structures, it becomes very expensive to replace or fix a certain defective piece. For multilayered composite material, the fabrication of the layered material may 
be susceptible to delamination between layers. A delamination is a portion of the layer that is not fully bonded to the adjacent layer. The delamination can reduce the strength of the material and cause failure to occur faster. Delamination is a continual problem in the fabrication and the performance of composites and can eventually lead to catastrophic failures. An example of this is the Space Shuttle Columbia disaster, which occurred in 2003. After extensive research on the disasters, it was determined that the reinforced carbon/carbon panel was impacted by a main propellant foam piece which could have caused enough damage for the panel coating to crack and delaminate internally. This would eventually cause the reinforced carbon/carbon to burn up.

Therefore, studying the behaviors of delaminated composite sandwich specimens is important for various applications. By being able to predict the effects of a delaminated composite, engineers can better understand the consequences of having a structure delaminated. This thesis will not only study the vibrational effects of a delaminated composite, but also increase knowledge of composite applications.

\subsection{Previous Work}

There have been numerous studies on model development for analyzing composite sandwich material with debonding parameters; however, the effects of applying a damage arrestment device on a delaminated composite sandwich have been investigated by very few researchers. Reviewing previous work on similar research is of great value to aid in manufacturing methods, test procedures, and testing apparatuses. Researching previous work can also help an investigator know what has worked in the past and what has failed, which can save time and money.

In J. Jakobsen's paper ${ }^{7}$ that addresses the peel-stopper, the purpose of the peel stopper is to effectively stop the development of delamination by rerouting it to a predefined zone in the structure. The peel-stopper design, as can be seen in Fig. 3, was experimentally tested with various types of materials to make sandwich beams that were subjected to a three-point bending load. 


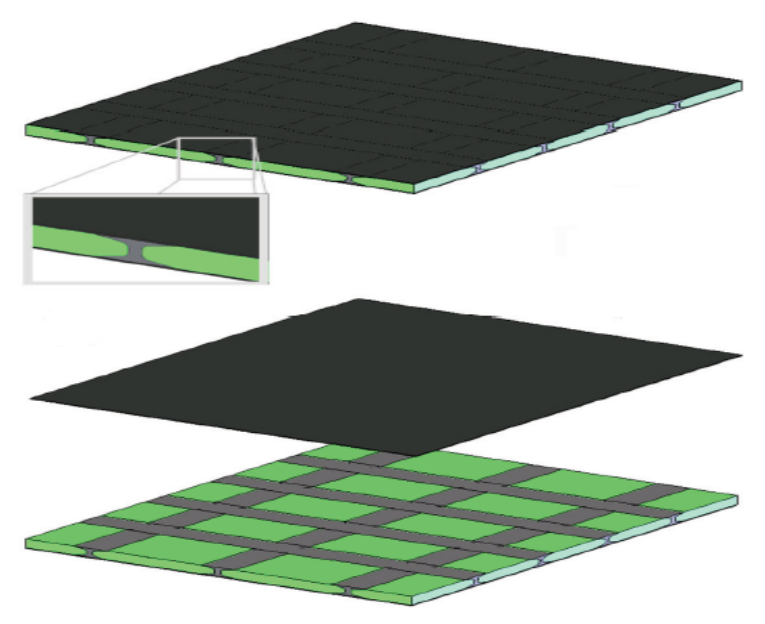

Figure 3: Jakobsen's peel-stopper design for stopping delamination in the face-core interface.

This design was successful in stopping delamination in the face-core interface. What the researchers found was that the peel-stoppers that worked the best were made with materials with large straining capabilities i.e. ductile materials. The experiment concluded that the ability of the peel-stopper to effectively stop crack propagation was highly dependent upon the stiffness of the inserted wedge. This finding of Jakobsen's is of high interest to the work discussed in this paper; the material used as the wedge makes a large impact on the end result. Jakobsen also found that the peel-stopper only worked when it was embedded throughout the entire specimen, connected to both facesheets. Joachim Grenestedt's ${ }^{8}$ research on peel-stoppers for sandwich structures found that the specimen could be fabricated with the peel-stopper inserted without affecting the overall structural strength of the part. Grenestedt's idea of coming up with a new peel-stopper design arose from the failing of boat hulls when a small crack would make the skin debond and grow like a fatigue crack. Two main tests were performed: quasi-static mechanical tests were performed to verify the function of the peel-stopper, and the strength of the structure incorporating the peel-stopper. The results concluded that the peel-stopper design was as strong as a control specimen without an insert when faced with a bending load. However, the specimen with a peel-stopper was not as strong as the control specimen when subjected to a shear load. This paper concludes that peel-stopping devices should be placed in areas where shear loads are not too high because they have an adverse effect on the overall structural integrity of the sample. Again, this method uses an anti-delamination device that connects the two facesheets.

In terms of vibration, there have been several ideas and experiments conducted on eliminating the chances of fluttering occurring on a structure or increasing the natural frequency. The uses of smart structures or piezoelectric elements have been used as sensors or actuators for the active control of structures. The design of such active systems requires accurate models of the electro-mechanical 
interaction between the structure and piezoelectric sensors or actuators. Piezoelectric elements are usually bonded to the top and bottom of the structure or are embedded in the composite beam as continues layers. Figure 4, show the set up of one set of actuators on the top and bottom on a composite beam.

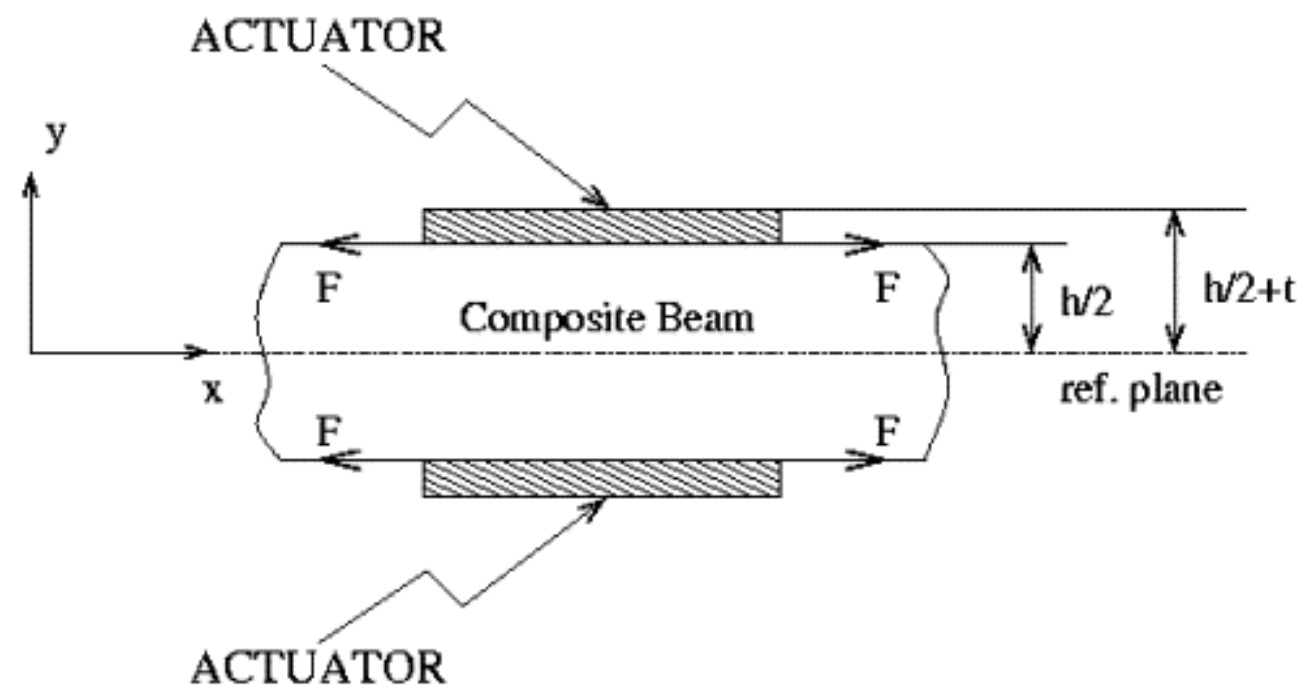

Figure 4: Illustration of how actuators are mounted on a composite structure to alter natural frequency.

Already in the 1970s, Rammerstorfer proved that it is possible to increase the first natural frequency and buckling load of plates by application of optimal fields of initial stresses ${ }^{9}$. Waisman and Abramovich investigated a wide range of cases considering the stress stiffening effect of a smart piezolaminated composite Timoshenko type beam. The model had one set of piezoelectric actuators bonded on both sides of the beam. It was demonstrated that the stiffness of the beam could be actively altered using the piezoelectric bonded actuators, yielding significant changes in the natural frequencies and mode shapes of the beam. One advance of having an active control over a passive control on a structure is the weight implemented on the structure. Typically an active control weighs less than increasing the stiffness by implementing a dampener or stiffener into the structure. However, the complexity and testing considerations are greatly increased when applying an active controller to alter or increase the natural frequency of the composite.

In the study done by W.M. Ostachowicz and S. Kaczmarczyk, the dynamics of a multi-layer composite plate with delaminations was analyzed under aerodynamic loading ${ }^{8}$. Their research suggested that the vibration response of the panel in flutter can be radically modified by means of stress-controllable shape memory alloys fibers embedded within the composite panel. Similar phenomena were investigated by Strganac and Kim. They studied vibrations of a damaged cantilevered composite beam. Aerodynamic loads were modeled using the so called "piston theory". Xue and Mei proposed a model of composite isotropic plates, and conducted studies of undamaged plate dynamics. Ostachowicz and Cartmell developed a model of a multi-layered composite plate reinforced using shape memory alloy wires, and 
Ostachowicz and Kawiecki investigated flow-induced vibrations of structures without shape memory alloys wires. Mechanical and physical properties of shape memory alloys strongly depend on temperature and initial stress. Changes in temperature and initial stress involve changes in the volume fraction of martensite, a very hard form of steel crystalline structure, in the alloys. During the martensite transformation the recovery stress appears.

Similarities can be drawn from the Ostachowicz and Kaczmarczyk experiment to this study where material was embedded into the structure to alter the natural frequency. Unlike Ostachowicz and Kaczmarczyk, heat will not be applied to the structure to change the initial stress of the composite. However, various damage arrestment devices will be implemented into the carbon fiber sandwich composite to study the effect and material properties under a dynamic loading.

In terms of damage arrestment devices, there have been various studies conducted to optimize the geometry and manufacture method for creating the damage arrestment devices. For this research, the damage arrestment device known as shear keys was considered in delaminated composites by Dr. Mitra, and Dr. Kasper ${ }^{12}$. The shear keys are a way to prevent or delay the propagation of the delamination of the structure. Since then various students at California Polytechnic San Luis Obispo have continued to develop shear keys. Various students who include Dominic Surano ${ }^{13}$, Richard Balabat ${ }^{14}$, and Tony $\operatorname{Tran}^{15}$ have researched different shapes and geometries for the shear keys. Their results concluded that a shear key geometry of a semi-circle with a radius of 0.25 inches was applicable for various structure applications.

\subsection{Objective Overview}

This project originally started with a grant from the California Central Coast Research Partnership (C3RP) under Award \# N00014-08-1-1209, which focused on the investigation of the effects of seawater degradation on a fiberglass sandwich composite under a shear loading. Once this was determined, methods of strengthening the composite panel to reduce the degradation effects would be examined. This research by Tomas Woo and Mike Jacobson ${ }^{16}$ led to the development of the damage arrestment devices called shear keys. Shear Keys were created as a method to strengthen the composite panels against shear force loading condition. The Shear Keys were made by placing strands of fiberglass and epoxy in a semi-circular mold, thus creating unidirectional fibers. The addition of the shear keys showed promise in the experimental specimens. The shear keys appeared to increase the ultimate load and shear capacity of the structure.

A similar investigation regarding damage arrestment devices led to the study of utilizing shear keys to prolong delamination in terms of bending. Richard Davis studied the effects of shear keys on 
sandwich composite beams under four-point bending. This research studies the use of a damage arrestment device that connects the carbon fiber facesheets to the foam core to determine whether the structural integrity of the sandwich beam is increased. Two control beams were utilized for comparison: one with no defects and another with a one- inch delamination introduced at the face-core interface. The shear keys were added in two different configurations to potentially stop the delamination propagation and increase the ultimate strength. In the first configuration, shear keys were placed 0.25 inches on either side of the initial delamination in the transverse direction and provided a significant increase in strength over the delaminated control beam. The second configuration had a shear key running along the longitudinal axis of the sandwich beam and resulted in a significant increase in ultimate strength over the delaminated control beam. Based on these investigations arose a need to further investigate the effects of shear keys on other structural loadings besides bending or shear loading.

The main objective of this research is to analyze the natural frequencies of carbon fiber/epoxy composite sandwich plates. These specimens will have similar dimensions and will consist of CF1803 bidirectional woven carbon fiber and LTM45EL epoxy matrix facesheets. However, a delamination will be placed between the carbon fiber laminate and the foam core for different samples. Two sets of composite specimens were analyzed during the experiment to determine the effects of various delamination locations. The two specimen sets consisted of a composite sandwich beam and the other was composite sandwich plate geometry. For each geometry set, delamination was intentionally place between the foam and facesheet in different locations. The different placements of the delamination reside horizontally on the top, bottom, and middle of the composite beam and plate. However, for the plate case there are two vertical delamination cases that were placed in the middle and on the side of the composite sandwich plate. The delaminated composite specimens consisted of one-inch delamination regions running horizontally across the beam and plate, while the control specimens were without delamination between the carbon fiber facesheets and the foam core. When incorporating the damage arrestment devices known as shear keys inside the structure, two shear keys were placed along the edge of the delamination region. For these samples, the control shear key case did not have a delamination region while the second case incorporated both the shear keys and delamination. The main focus of this experiment is to determine the effects of delamination in terms of frequency and amplitude. These results will determine whether the location of the delamination increases or decreases the natural frequency or amplitude of the composite. With the values from the vibration software, a comparison between the experimental and theoretical values from the finite element software COSMOS can be accomplished. The comparison will determine the fidelity of the finite element program to the experimental values of the composite plates. Along with determining the amplitude and frequency of each specimen, further studies will be made for each case to determine how the time domain is affected for the different composite cases. 


\section{Manufacturing Procedure and Experimental Setup}

For the experimental phase of this thesis there were three primary materials were used to manufacture all of the composite sandwich specimens. CF1803 bidirectional woven carbon fiber with LTM45EL epoxy matrix was used for the composite sandwich facesheets. The core of the sandwich comprised of a low density FR 6710 Last-A-Foam material. Finally the damage arrestment devices consisted of woven roving fiberglass with Aeropoxy matrix. It was very important to have consistencies among the lay-ups and the manufacturing of various composite specimens. Therefore, these materials were all tested to standards in order to receive accurate and reliable data. Once the material properties are obtained, the composites have consistent results, and are cured to an acceptable criterion; then the composite sandwich beams and plates can be manufactured. Along with manufacturing the sandwich specimen, the material properties from the three primary materials will assist in modeling accurate finite element models for the numerical analysis.

\subsection{Mechanical Characteristics of the Carbon Fiber Facesheets}

To have a valid experiment and composite specimens it was essential to have a consistent fabrication method for all testing samples throughout the experimental phase. The materials used for the entire experiment were CF1803 bidirectional woven carbon fiber and LTM45EL epoxy matrix. This preimpregnated carbon fiber was one of two kinds available for the aerospace composite lab. The LTM45/CF1803 carbon fiber was chosen for several reasons. This type of carbon fiber has been previously used in the past when implementing damage arrestment devices and the curing cycle was within the acceptable region for the autoclave. During this experiment, the Cal Poly Aerospace Autoclave, provided by American Autoclave Corporation, was functioning and was available for this study. However, there were temperature limitations for this autoclave which limited the maximum temperature to $250^{\circ} \mathrm{F}$. A technician from the American Autoclave Corporation instructed us not to implement a cure cycle with temperatures exceeding $250^{\circ} \mathrm{F}$ while using compressed air inside the autoclave because that would be hazardous. If a cure cycle required a higher temperature, then nitrogen gas would need to be connected to the autoclave. Therefore, to save time and cost, LTM45/CF1803 carbon fiber was used for this experiment. 


\subsubsection{Cure Cycle and Lay-up}

The LTM45/CF1803 carbon fiber is an epoxy matrix resin composite produced by Advance Composite Group which recommends a certain bagging arrangement and a curing cycle. The recommended bagging arrangement required various materials in order to have a valid part. The product description handout fully described the application of each layer and its location in the composite lay-up. Figure 5 illustrates the lay-up of the LTM45/CF1803 carbon fiber laminate.

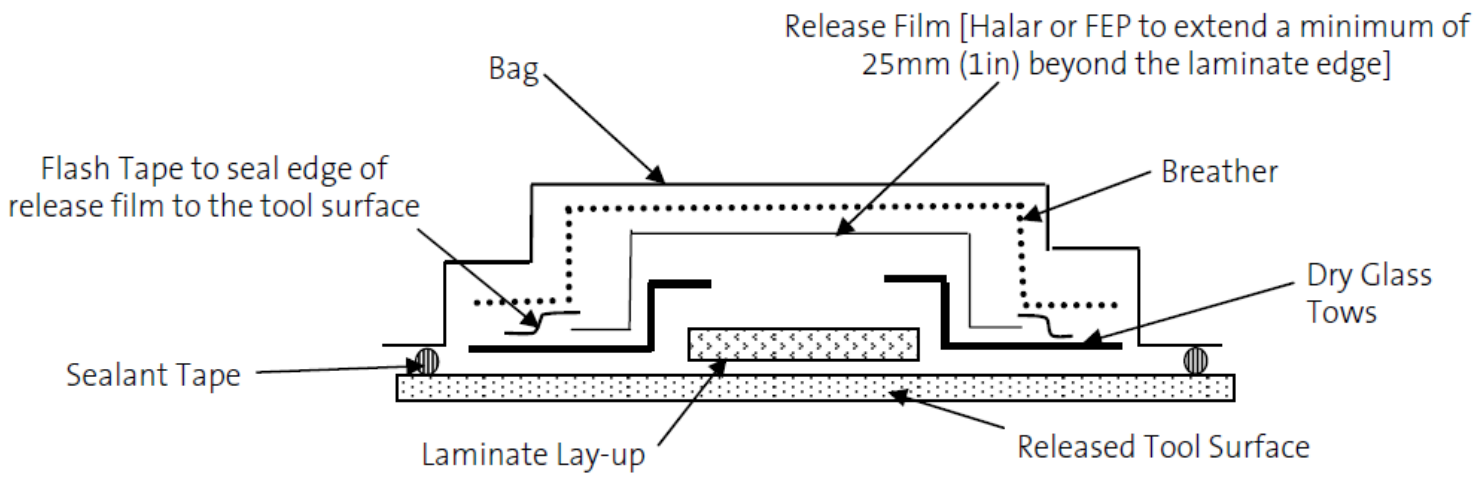

Figure 5: LTM45/CF1803 layup provided by the Advance Composite Group ${ }^{21}$.

The lay-up included a vacuum bag, a breather fabric, release film, dry glass tows, and sealant for the vacuum bag and the released tool surface. However, there was one lamination on acquiring the materials recommended for this lay-up. The only material that was not available, for this experiment, was the dry glass tows for the lay-up. Nonetheless, the composite lay-up continued without the dry glass tow for the entire experiment. The next issue was determining what type of breather was best suited for this composite in order to have the best curing cycle. In the Cal Poly aerospace lab there were various breathers that could have been used for this lay-up. Therefore, experiments were conducted to determine which breather best suited the carbon fiber material.

The curing cycle for the LTM45/CF1803 carbon fiber was also obtained from the manufacturer's website. The specifications for an autoclave cure explained that the pressure inside needs to rise to the maximum pressure at a rate of 5 psi per minute. For California Polytechnic State University's aerospace autoclave, the maximum pressure inside the chamber was limited to $70 \mathrm{psi}$. The vacuum bag, which was placed over the specimen, was required to have a vacuum pressure of at least $25 \mathrm{in} . \mathrm{Hg}$ for every specimen. The temperature for the curing cycle needed to increase at a rate of $5^{\circ} \mathrm{F}$ per minute until it reached a value of $150^{\circ} \mathrm{F}$. Once both the temperature and pressure have met the set requirements, both parameters must idle in their respective values for 16 hours. Once 16 hours have passed, the temperature must decrease to a set value of $73^{\circ} \mathrm{F}$ at a rate of $5^{\circ} \mathrm{F}$ per minute. It was recommended that the pressure in 
the autoclave be reduced to atmospheric pressure at any rate that does not exceed 10 psi per minute. An illustration of this cure cycle can be seen on Fig. 6 which shows the trend line of the curing cycle.

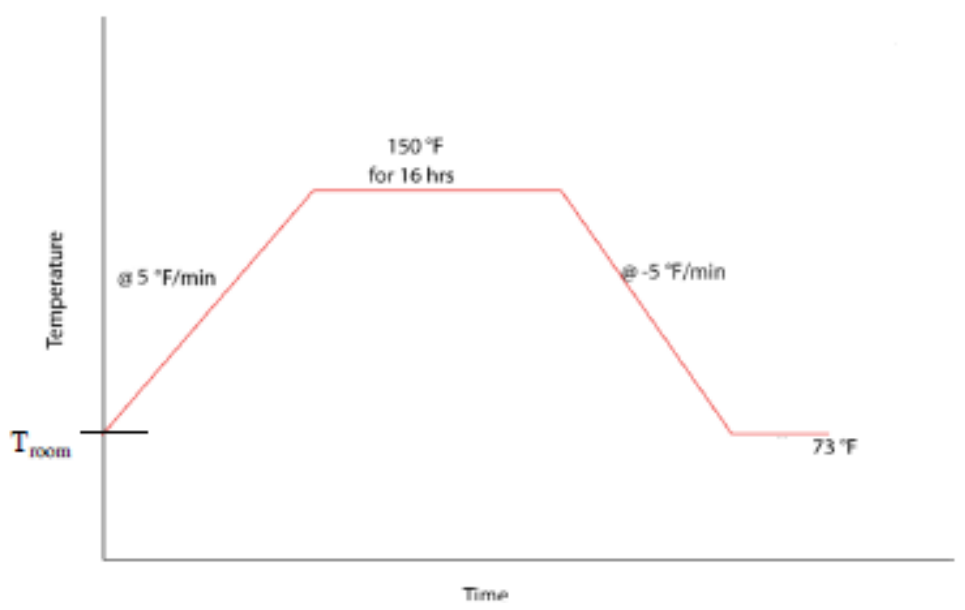

Figure 6: Curing cycle provided by the Advance Composite Group for the LTM45/CF1803 carbon fiber.

One important difference between the theoretical curing cycle and the actual curing cycle inside the autoclave it that the steady rate for 16 hours was not precise. When creating a recipe for the curing cycle, the autoclave did not allow tight tolerances in temperature. While idling for 16 hours the temperature inside the autoclave had a $\pm 5^{\circ} \mathrm{F}$ for the curing process. The temperature setting also had a problem decreasing at a rate of $5^{\circ} \mathrm{F}$ per minute even though it was programmed to the exact setting. Typically the cure cycle decreased at a rate of $1^{\circ} \mathrm{F}$ per minute.

\subsubsection{Carbon Fiber Facesheet Fabrication Method}

Several breather materials that could have been used in the lay-up of the carbon fiber laminate; therefore, a common method for all testing was established. In the aerospace composite lab there are several breathers that students have access to which include a cotton based breather, multimedia, and a perforated vacuum bag. These breather materials were all fabricated in order to determine which breather would give the best results in terms of tensile strength. Each lay-up consisted of two sheets of $13 \mathrm{x} 13$ inches of airtech porous material, a 22 x 22 inches of vacuum bag, two small 2 x 2 inch cotton sheets, two 12 x 12 inches of lamina sheets of LTM45/CF1803 carbon fiber, a single 14 x 14 inches of breather sheet, and a custom made release tool surface. Figure 7 depicts the materials used to lay-up a carbon fiber specimen with the breather being a perforated release vacuum bag. 


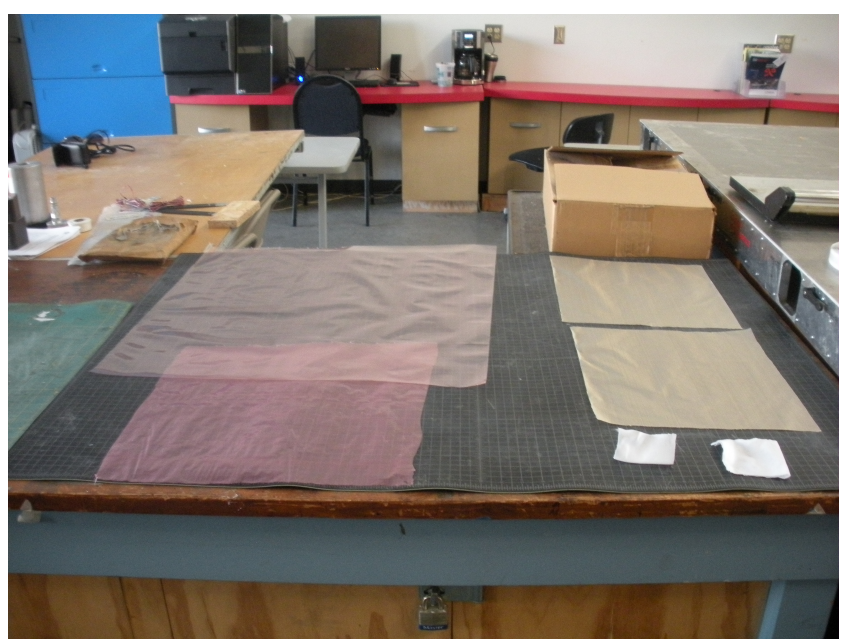

Figure 7: Material used to manufacture a carbon fiber sample.

The assembly of the fabrication was as follows: vacuum bag, perforated release vacuum bag, porous material, two layers of bidirectional CF1803 prepreg carbon fiber, and porous material made out of fiberglass. The porous material was essential because it allows for resin to flow through its layer and fully saturate the prepreg carbon fiber. The perforated release vacuum bag sheet was used to provide air initially inside the bag to be drawn off by the vacuum source, which creates a better vacuum. The two cotton patches are used at the base of each vacuum pump suctions located on the customized release tool surface. One cotton sheet is placed on the top surface of the release tool surface which leads to a built-in quick connect that is used to connect one side of the vacuum pump. The second cotton patch was placed on the opposite corner of the release tool surface under the second vacuum pump connection. These two square cotton sheets are used as a precautionary material in case any resin escapes from the carbon fiber and into the vacuum pumps. The goal was to prevent resin from clogging the pipes.

The bidirectional CF1803 prepreg carbon fiber was stored inside a freezer near the lab in order to preserve the material. The take-out time for this carbon fiber was 3 days; however it was vital not to work under high temperature conditions. In hot conditions the resin will seep out and will add complexity when combining sheets of laminas together. The carbon fiber roll was laid on top of a flat surface and weights were gently applied on the edges to prevent the roll from contracting back; an illustration of this is shown in Fig. 8. The carbon fiber was then cut by a rolling blade to form a sheet of $52 \times 12$ inches. In this procedure four pieces of carbon fiber were produced from this long piece. Once again these sheets were cut with a rolling blade with the use of a straight edge and a ruler. Even though the CF1803 prepreg carbon fiber was bidirectional woven fiber, there was a dominant fiber angle on each side of the carbon fiber. Having a different fiber orientation will change the material properties, therefore all the experimental specimen had the dominant fiber in the 0 degree orientation for the outer fibers. 


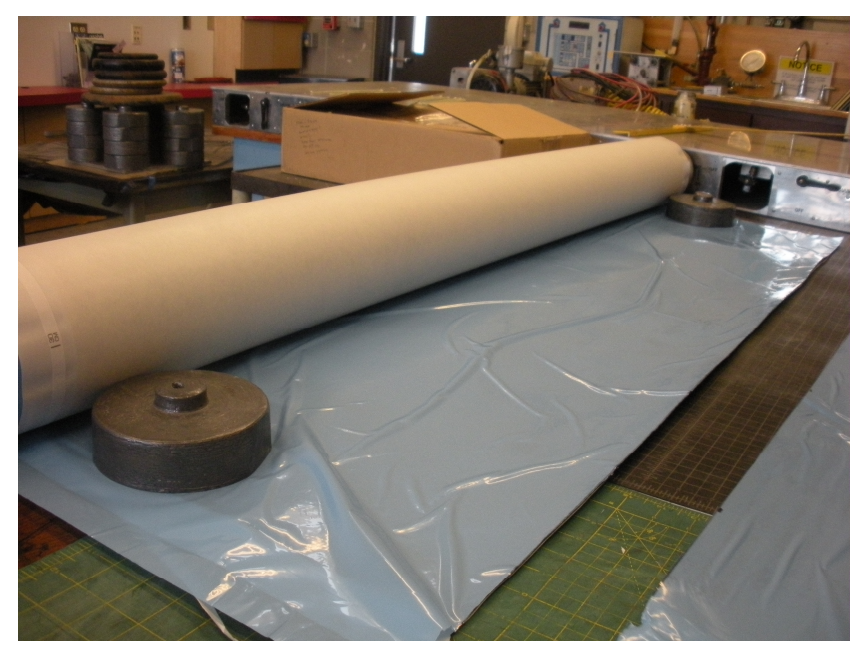

Figure 8: Prepreg carbon fiber role taken out of the Cal Poly Aerospace freezer.

The materials were arranged as described in the previous paragraphs. Once the materials were placed together, the rolling pin was used to remove the voids that might be created during the lay-up process. The vacuum bag was then applied and sealed onto the release tool surface using gum tape. It was vital that all creases were eliminated; otherwise air can escape from the vacuum bag, which will prevent the vacuum from reaching its minimum value of $25 \mathrm{in}$. $\mathrm{Hg}$. If there was an excess amount of vacuum bag, a pleat was made between the excess vacuum bag and release tool surface. Once the lay-up was complete, it was placed inside the American autoclave near the aerospace lab which is depicted in Fig 9. The hoses were then connected to the two connections on the tool surface to apply a vacuum on the specimen.
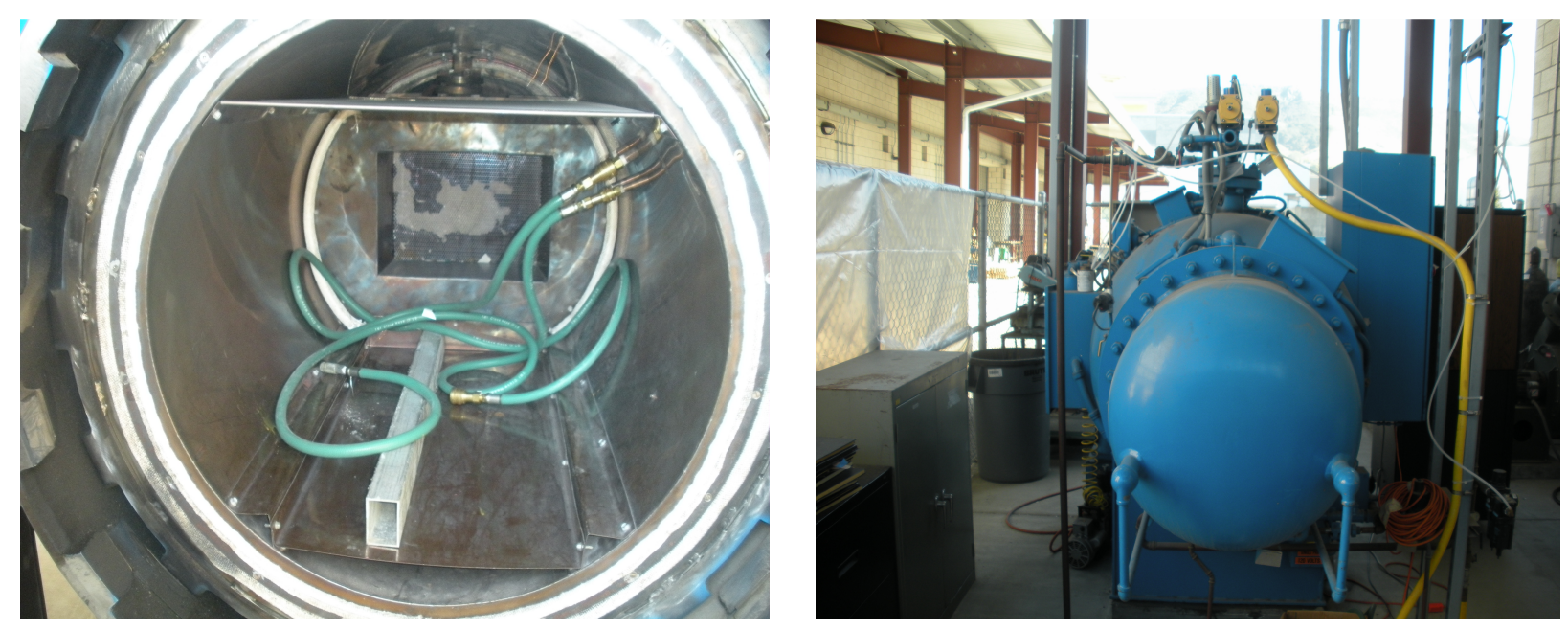

Figure 9: American Autoclave located near Cal Poly Aerospace Laboratory. 
Certain steps are required in order to activate the autoclave and select the proper curing cycle. Several steps include turning on the main power switches and computer, login as a student user, and selecting the cure control and specifying the correct curing cycle recipe. The curing cycle selected was created in the beginning of the experiment to reflect the cure that the advance composite group suggested. Templates were followed to select the correct criteria and to place correct values inside the recipe file. Once the recipe was selected, the autoclave door was opened, the composite specimen placed inside, and the vacuum hoses connected. Figure 10 shows the vacuum pressure gage and vacuum used for all the composite specimens. The vacuum needed to be at least $25 \mathrm{in}$. $\mathrm{Hg}$ before the door was closed in order to manufacture a good specimen. Before starting the autoclave, all specimens were left with the vacuum on to allow the specimen to debulk before starting the curing cycle. This process was done for 30 to 60 minutes for each part, and then the autoclave was turned on.
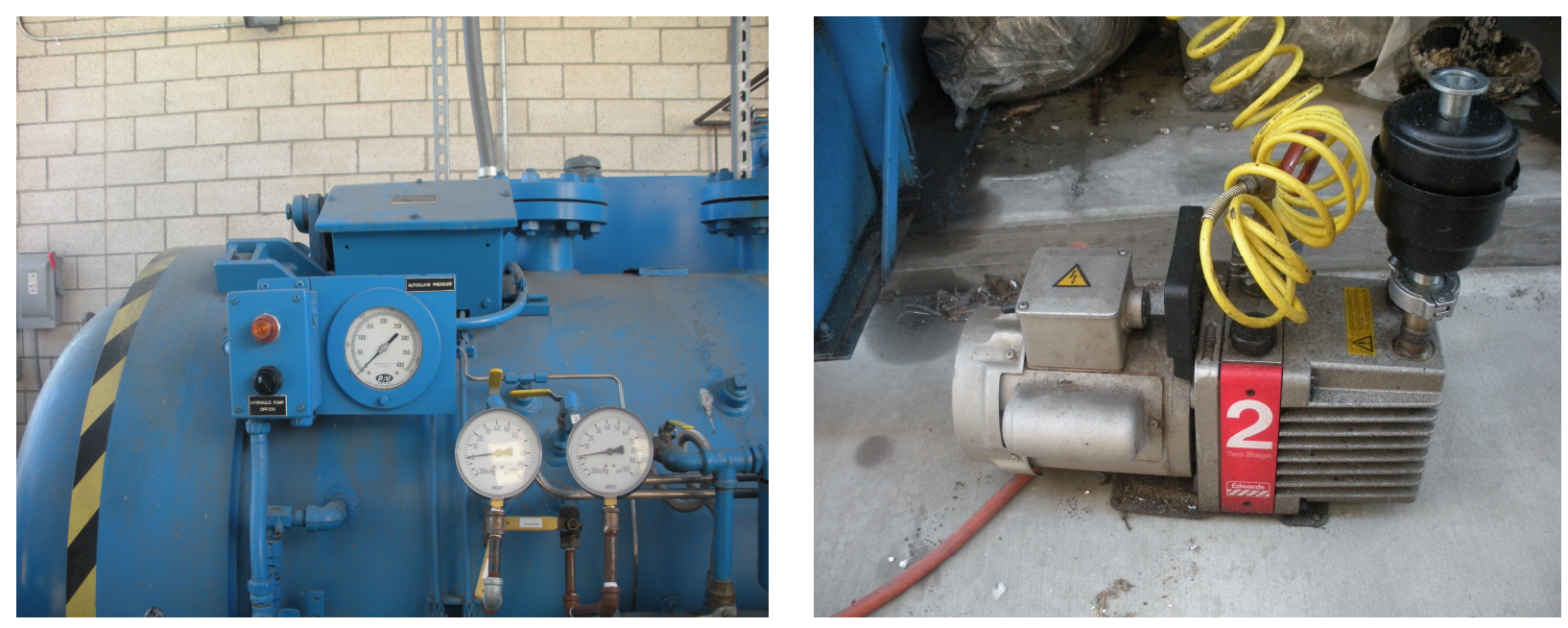

Figure 10: Pressure valves and vacuum pump located next to the American Autoclave.

The specimen was left overnight until the curing cycle was completed. At this point, the tool surface was carefully removed from the autoclave by disconnecting the vacuum connections. The carbon fiber composite was removed from the lay-up and the materials were thrown away, due to the fact that resin had saturated each material layer. If there was any resin resting on the tool surface, the surface was then cleaned with the use of a razor blade and wiped down with a towel sodden with acetone.

\subsubsection{Acquiring Elastic Modulus and Shear Modulus of the Carbon Fiber Facesheets}

Tests were implemented on the carbon fiber lay-ups with different breathers in accordance to ASTM standard D3039, which are the standards used to find mechanical characteristics of composite 
samples. This standard requires that samples be cut to $10 \mathrm{x} 1$ inch dimensions, as seen in Fig. 11. Cutting the specimens to the desired dimensions required a tile saw with an accurate measuring device to ensure repeatability between samples. When the specimens were cut, the measurements and orientations of each sample were checked so that an accurate comparison of results could be attained. A slight change in the geometry or orientation would weaken the fidelity of this experiment. Therefore, it was important that the necessary attention was placed on cutting the composite specimens.

Aluminum tabs, also seen in Fig. 11, were machined and sanded at the Cal Poly machine shop. The aluminum tabs were cut to 1 by 1 inches with a thickness of .025 inches. The edges were later sanded down and smoothened out to eliminate any chances of not having a flush surface to surface contact with the carbon fiber specimen. These tabs were then cleaned and attached to the carbon fiber samples with a resin-epoxy adhesive, 3M Scotch-Weld Epoxy Adhesive DP-460.

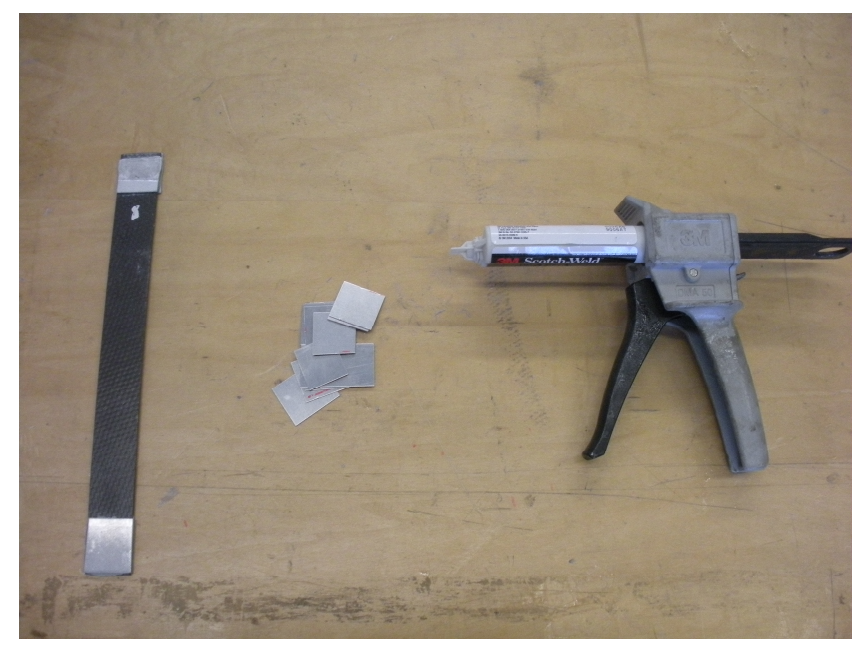

Figure 11: 3M Scotch-Weld Epoxy Adhesive DP-460 used to attach aluminum tabs to specimens.

The samples were then placed inside a tetrahedral press in a lay-up that consider of a smooth steel plate, porous sheet, seven carbon fiber specimens, porous sheet, and another smooth steel plate. The decision was made to place the carbon fiber samples inside the press to insure a uniform pressure throughout the seven samples. The tetrahedral press was then programmed to a pressure $1000 \mathrm{lbs}$. $/ \mathrm{in}^{2}$ and a temperature of $100{ }^{\circ} \mathrm{F}$ for 24 hours. Once the samples were cured, they were ready to start the tension testing in the Instron machine.

The standard tension testing method was then programmed into the Instron machine such that each sample was tested in exactly the same way. Each specimen was put into the Instron jig so that the specimen was parallel with the grips and perpendicular to the floor, as seen in Fig. 12. 


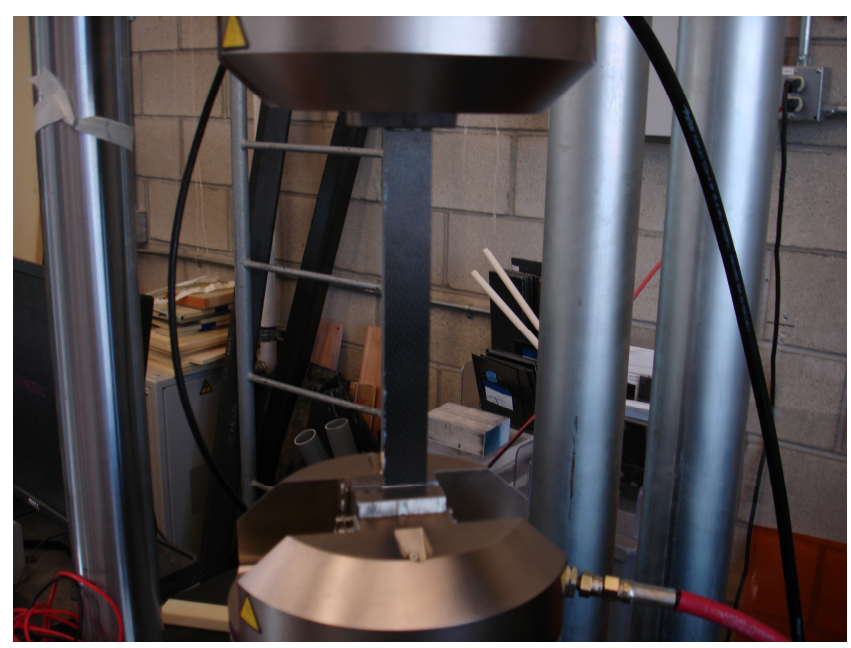

Figure 12: Tension test applied on a carbon fiber coupon using the Cal Poly Aerospace Instron machine.

The carbon fiber sample was then loaded in tension until it broke or until the load dropped by 40 percent. The Instron machine records all testing data: stress, strain, load, elongation, and ultimate stress. The data for each sample was recorded on the computer, and then analyzed to obtain the Young's modulus. A total of seven specimens were conducted to acquire a statistically good average for the Young's modulus. The Young's modulus was found by graphing the stress against the strain and finding the value of the slope using the computer program Matlab. This test was repeated for the three different types of breather lay-up with each set of composite having at least seven different samples of $1 \mathrm{x} 10 \mathrm{inch}$ specimens.

\subsubsection{Poisson's Ratio of the Carbon Fiber Facesheets}

Poisson's ratio was determined by using strain gages attached to a P-3500 Strain Indicator and an extensometer strain gage attached to the Instron machine. Strain gages were placed parallel and perpendicular to the length of the specimen. The gage aligned in the parallel direction was understood to be in the longitudinal direction. The gage aligned in the perpendicular direction was said to be in the lateral direction. Each gage was attached to two lead wires that were connected to the P-3500 Strain indicator. To acquire the Poisson's ratio, both longitudinal and transverse strains are necessary. Only one gage was in the transverse direction while in the longitudinal direction an extensometer was placed to acquire the strain. The extensometer was connected to the Instron machine in order to record the data onto the computer. The P-3500 recorded the strain in the longitudinal direction during the tension test and the extensometer strain gage was added to each sample in the lateral direction and loaded.

The strain gages used in this research are Vishay Micromeasurements $120 \Omega$ resistance gages with a model number of C2A-13-250-W-120. Strain gages were applied following the technique outlined 
in the Student Strain Gage Application Manual as written by Vishay. The area where the strain gages were going to be applied was cleaned using acetone and then the alkaline base cleaner provided by Vishay Micromeasurements. A paper towel was dampened with acetone and used to clean the surface of the specimens. Next, a few drops of cleaner were applied to cotton swabs which were then used to finish cleaning the surface of the specimen. The swabs were run in a single direction to avoid picking up debris and re-depositing it in the application area. M-Bond 200 adhesive was used to apply strain gages to the test specimens. The adhesive consists of a two-part epoxy as shown in Fig. 13.

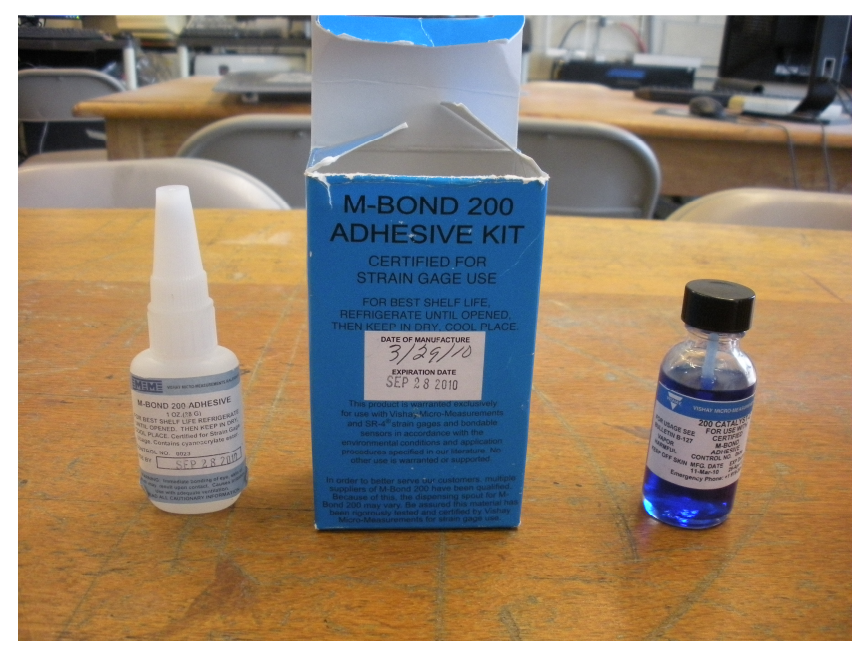

Figure 13: Chemical components used to connect strain gages to fiberglass and carbon fiber test samples.

Before the strain gage was applied to the carbon fiber specimen, the placement was drawn out on the specimen using a pencil. It was very important that the marking was exactly 0 degrees along the specimen, or perpendicular to the carbon fiber fibers. The strain gage was then placed on scotch tape in order to place the gage on the marking and to have an effective bonding between the two-part epoxy. The catalyst liquid was placed on the strain gage while the other epoxy component was placed on the location where the strain gage would reside on the composite specimen. The strain gage was carefully placed on the carbon fiber specimen and pressure was applied. The wires that connect to the strain gage were then used to connect to the P-3500 Strain Indicator. Figure 14 shows the layout of how the composite specimen, strain gage, and strain indicator were connected. 


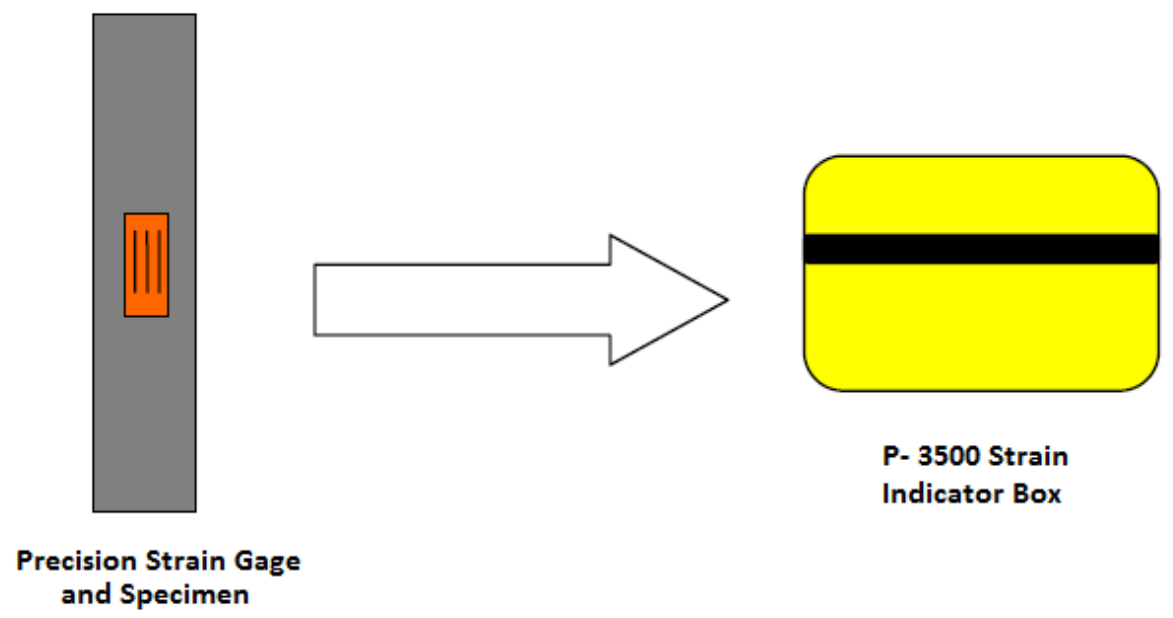

Figure 14: Illustration of the connection needed in order to collect data from a Strain Gage.

The following series of loading tests were performed on specimens with strain gages to determine the proper full-scale gage length and calibration point settings for the utilized strain gages. Bluehill 2 was used to record longitudinal strain gage data and the lateral strain gage data were recorded using the Strain Indicator box which was taken during the same tests. The method used for finding the Poisson's ratio of the facesheets follows ASTM E132 testing standard. This format follows the exact fabrication and test method as the elastic modulus of a carbon fiber sample standard. The only difference between this test and the previous test was that a strain gage was added in the transverse direction in order to capture the two-dimensional behavior of the laminate.

A series of tests were performed on an Instron 8801 servo-hydraulic testing system to determine Poisson's ratio of the carbon fiber test specimens. Tests were controlled by Bluehill 2 software on an attached controller and data collection PC. Poisson's ratio was defined as the negative of the transverse strain divided by the axial strain of a material under an applied load. These tests were done for several specimens while using the same techniques and procedures as in the elastic modulus procedure.

\subsubsection{Density and Volume Fracture of the Carbon Fiber Facesheets}

Facesheet density was relevant to this research because during the finite element analysis, the mass of each element was essential in determining the natural frequency of the model. Experimentally the values of density are found by taking a sample of the material and performing various volume and mass calculations to find the density. To find the density of the LTM45/CF1803 carbon fiber facesheets, the sample that was already cured was cut to a $1 \times 10$ inch dimension and re-measured with a micrometer to determine length, width, and thickness of the specimen. The weight was then measured by using the 
weight scale provided by the laboratory. This procedure was repeated for all the carbon fiber samples before they were tested for elastic modulus to ensure statistical accuracy. The density was then found by dividing the mass of the sample by the volumes of the sample.

A series of weight tests were done for various carbon fiber samples. This testing was done in order to ensure that the fiber and matrix content was consistent across several different layups. The oven that was used and the end result can be seen in Fig 15.
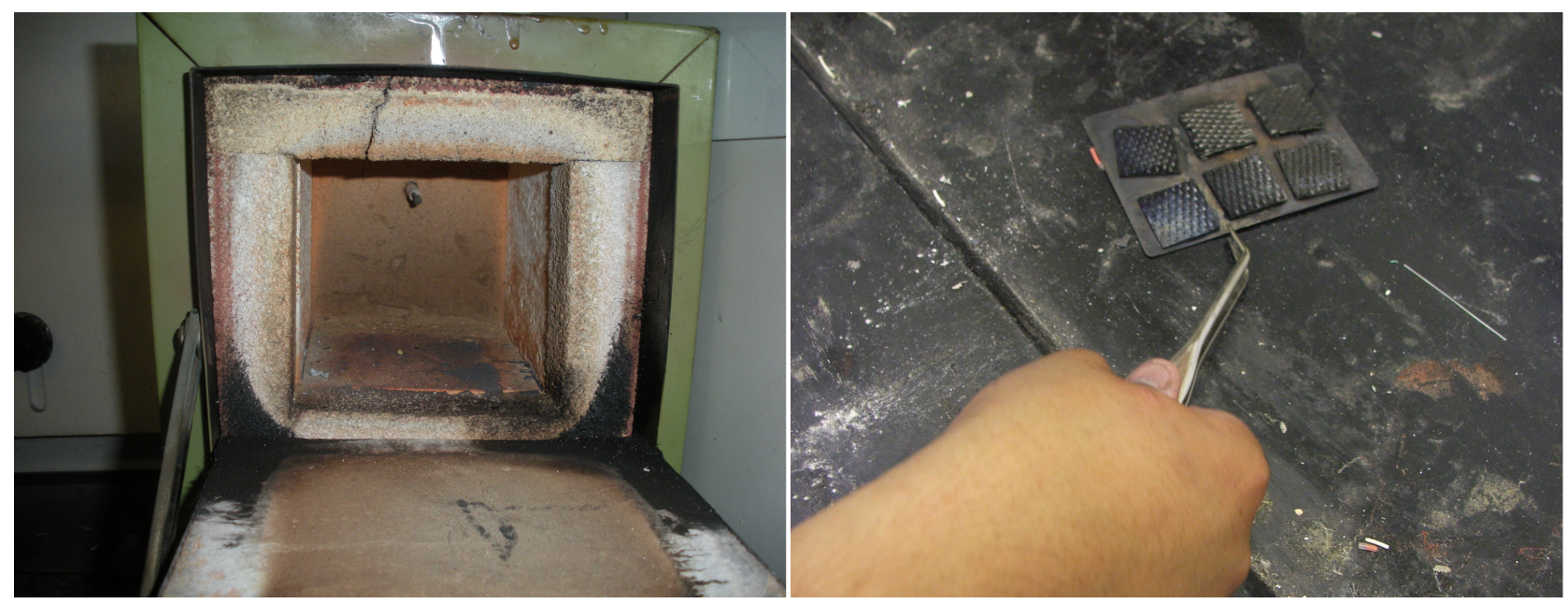

Figure 15: Cal Poly Aerospace oven which is used in conducting burn test on composite samples.

The burn test was based on a simple 3 step process. First a 1 x 1 inch specimen was weighed and then the specimen was placed in a small ceramic oven to separate the resin from the fibers. Once all of the resin was gone, all of the fibers that remained were removed from the oven and weighed. The one-inch square specimens were baked in the oven for approximately 30 minutes at $900^{\circ} \mathrm{F}$. This test was repeated for five samples in order to have a good average.

\subsection{Mechanical Characteristics of the Polyvinylchloride Foam}

The second important material used for the experiment was the FR 6710 polyvinylchloride Last-A-foam. Foam has been used for various applications in the aerospace industry and in the naval industry. Foam was used to increase the strength of the material without greatly increasing the cost or weight of the structure. FR 6710 polyvinylchloride Last-A-foam was chosen for all the composite sandwiches in the experiments to be consistent with the other experiments that have been conducted on the delaminated composite sandwich specimens at Cal Poly San Luis Obispo. In order to conduct a comparison between a numerical analysis solution and experimental data, a finite element model needed 
to be properly developed. The first step in creating this model was to gather the material properties for all materials which included the polyvinylchloride foam.

\subsubsection{Last-A-Foam Cutting Method}

With the assistance of the aerospace lab technician, the large sheets of FR 6710 polyvinylchloride Last-A-foam were cut down into $12 \mathrm{in}$. x 12in. pieces. The thickness of the foam was not altered and was kept at the standard specification of 0.5 inches. To cut the foam specimens to their desired geometry the foam was placed on a table saw and cut to specifications. Over 40 square specimens were created in order to have enough foam to conduct the entire experiment on dynamic analysis and on the material properties for the foam.

In order to acquire valid material properties, the test specimen was made from polyvinylchloride (PVC) rigid foam and was cut to a 2 in. $\mathrm{x} 2$ in. square cross-section. Extra care was taken to ensure the loaded ends of the specimen were parallel to each other and perpendicular to the sides. If samples contained any defects it was imperative that the sample be discarded and a new one made. The foam segments were only 0.5 inches thick which meant that layers had to be stacked in order to obtain the proper dimensions according to the testing standard. Figure 16 shows how the foam was cut from the larger sheet with a tile saw. Before starting the actual compressive testing, all samples are measured with a precision measuring device with a tolerance of $\pm 1 \%$ of the total dimension. All dimensions are recorded three times and averaged to obtain an average dimension.

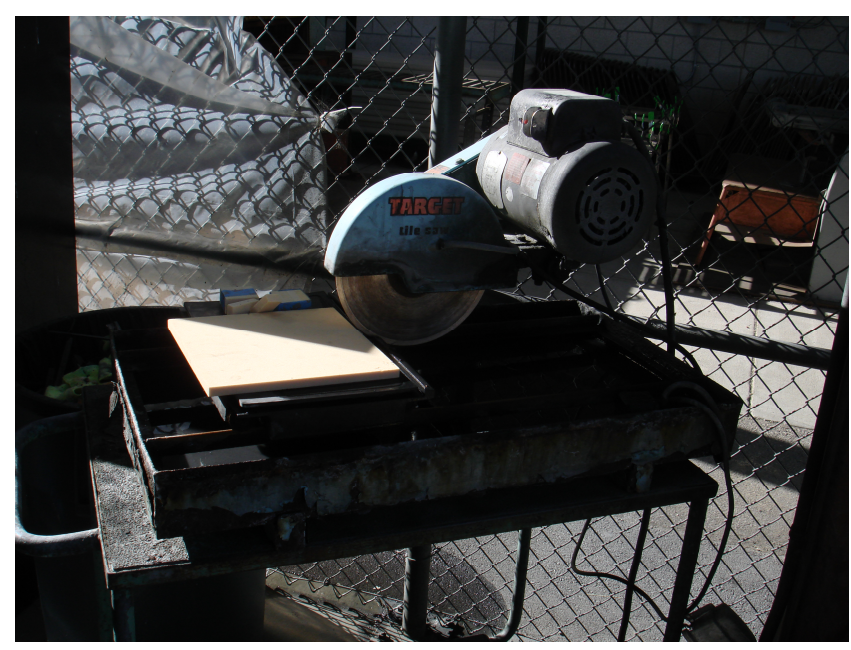

Figure 16: Tile saw used to cut smaller Last-A-Foam pieces to conduct material property tests. 


\subsubsection{Material Properties Set-up}

The test method for obtaining the PVC foam mechanical characteristics follows ASTM D1621 standard. This test standard is the test method for finding compressive properties of rigid cellular plastics. This test method describes the procedure for determining the compressive properties of rigid cellular materials, particularly expanded plastics. In this procedure, data was acquired and a complete loading deformation curve was obtained, which yields the compressive stress at any load as well as the effective modulus of elasticity.

The specimen was then positioned in the Instron machine that was equipped with two parallel plates used to compress the test specimen, ensuring that the sample was centered in the plates so that the load was uniformly distributed over the entire specimen. The PVC foam pieces were first placed between the two parallel plates perpendicular to the rise of the foam. The specimen was then compressed at a rate of 0.1 inch per minute for each inch of specimen thickness. The samples loaded in the Instron machine between the parallel plates are seen in Fig 17.

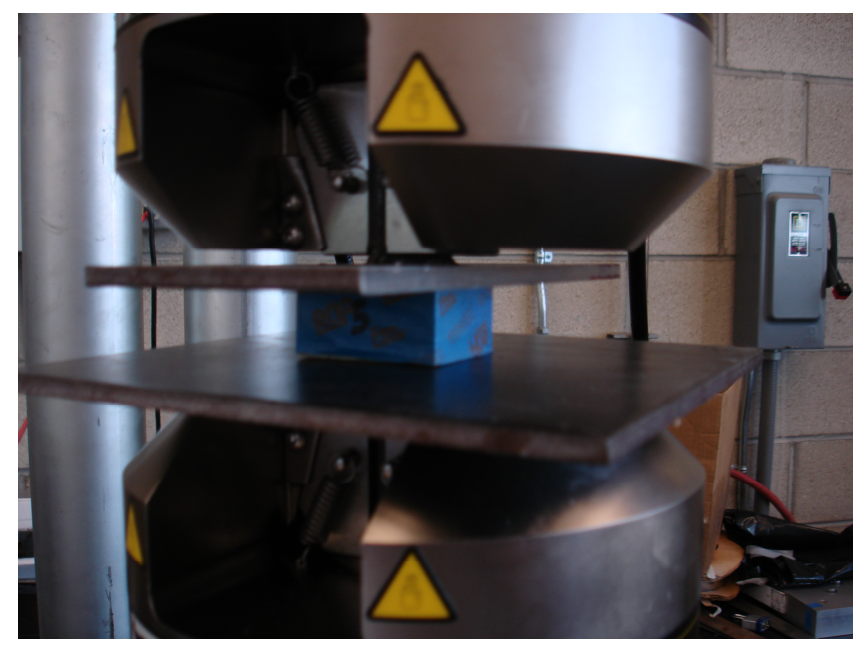

Figure 17: Compression test applied to the Last-A-Foam specimen to acquire the compressive modulus of the foam.

The load-deformation curve was then generated and the mechanical properties were obtained. The sample was compressed until the specimen had compressed by $13 \%$ of its overall thickness. After the samples were tested, the load-deformation curve was generated by the Instron machine which allowed for the elastic modulus to be determined. The elastic modulus was calculated by using the following equation from the testing standard. The test was then conducted with the foam piece being compressed parallel to the rise of the foam. By conducting the experiment parallel and perpendicular to the rise of the foam, this ensured that the elastic modulus and shear modulus were obtained for each direction of the foam. 


\subsection{Mechanical Characteristics of Fiber Glass Shear Keys}

Damage arrestment devices (DAD's) have two structural effects when placed in composite sandwiches. DAD's are meant to increase stiffness to the structure while not increasing the weight of the structure. The purpose of placing DAD's between a composite facesheet and core, is to increase the bonding area of the structure. This in turn will prevent or prolong the delamination process of the foam and facesheet. This experiment used Woven Roving 18oz Fiberglass as the material for DAD's. Fiberglass provides a high strength to weight ratio and has been used in previous studies of DAD's.

\subsubsection{Fiber Glass Shear Keys Fabrication Method}

The DAD specimens that were fabricated from Woven Roving Fiberglass strands were placed in a unidirectional orientation. These strands were removed and separated from the woven fiberglass role and cut into lengths of 15 inches, which were placed into a premade mold. A single DAD was composed of 15 strands of fiberglass wrapped together by rubber bands. The mold was made of aluminum, held 22 bundles of fiberglass at a time, and created 0.125 inch radius semicircular DAD's known as shear keys. The matrix of the shear keys is composed of a two part resin which includes Aeropoxy PH3660 hardener and Aeropoxy PR2032 resin as seen in Fig 18. The mixture ratio for this matrix resin was 100 to 27 of resin to hardener by weight. The bundles of fiberglass were laid-up by hand using Aeropoxy resin. When laying up the fiberglass by hand, it was important to ensure that the fiberglass bundles were completely saturated with resin throughout.

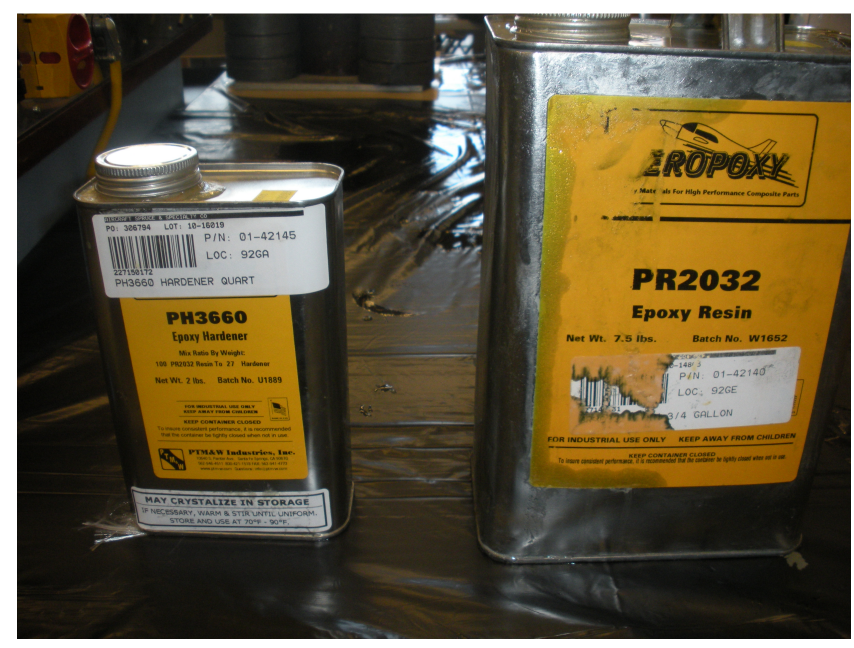

Figure 18: Aeropoxy hardener and resin used for the fiberglass shear keys.

Before placing the fiberglass bundles inside the aluminum mold, the mold was waxed by a 
releasing agent that allows the shear keys to be easily removed once they are cured. The wax was applied to the semi circular grooves by lightly coating them by hand. The fiberglass bundles that were saturated with resin were then placed inside the slots in the aluminum mold. To assist with getting the fiber glass bundles inside the grooves, the strands may be twisted into place. Once all the resin saturated fiberglass specimens were placed inside the mold, a sheet of $12 \times 12$ inch porous paper was laid on top of the shear keys. A flat aluminum surface was then carefully applied on top of the porous sheet to eliminate any chance of shifting the fiber orientation. Roughly $200 \mathrm{lbs}$ of weights were applied on top of the aluminum surface as seen in Fig 19.

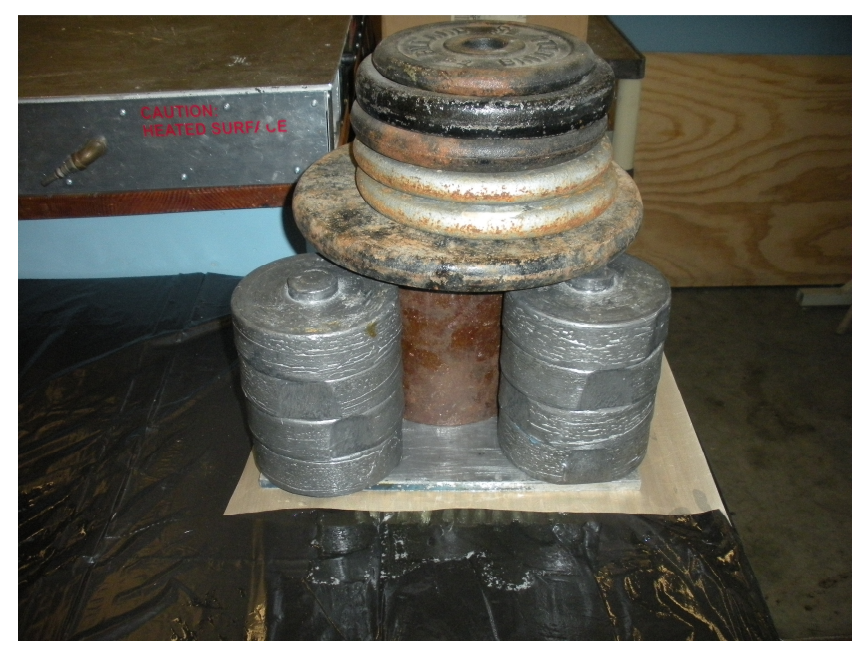

Figure 19: Distributed force applied onto the aluminum plate while the fiberglass shear keys cure.

The shear keys were left inside the mold for 24 hours in order to allow them to fully cure. Once the cure cycle had ended, the weights, aluminum plate, and the porous paper were removed from the specimens. The shear keys were then sanded down using 80 to 150 grit sandpaper. The sanding process can either be sanded by hand or a hand-held sander. Sanding was required to ensure the specimens had a radius of 0.125 inches and were embedded evenly throughout the top surface of the foam core. The orientation and the process of embedding the shear keys inside the foam will be discussed later in the composite shear keys section of the report.

\subsubsection{Acquiring Elastic Modulus and Shear Modulus of the Fiberglass Shear Keys}

The ASTM standard D3039 was once again followed to obtain the elastic modulus of the fiberglass shear key. Since the geometry of a shear key is a semicircle, these specimens could not be placed inside the Instron machine and tested. The test standard also required that the samples be cut to the 
dimension 10 x 1 inch. Therefore, a separate shear key plate was necessary. For this process the strands of fiberglass were aligned in a unidirectional fashion to simulate that direction of a shear key pattern. However, instead of bundling fiberglass together as would have been done on the shear keys, 30 parallel strands where placed side by side. The idea was to create a single lamina of unidirectional fiberglass strands. Weight was applied to the ends of the fiberglass to prevent the fibers from moving from their 0 degree orientation. Aeropoxy matrix was then applied to the fiberglass strands until each fiber was saturated with resin. Weights were carefully placed onto the composite to apply pressure during the curing cycle. After several days the cured plate was then cut to the desired dimensions with a tile saw. Strips were cut in the direction parallel and perpendicular to the fiber direction in order to obtain the material properties of each direction. Aluminum tabs were applied to the fiberglass specimens in a similar manner as the carbon fiber specimens.

Both sets of fiberglass strips were marked in order to recall the orientation of the direction of the fibers. This ensures that each set has the same characteristics when the specimens are tested on the Instron machine. The standard tension testing method was then programmed into the Instron machine to guarantee that each sample was tested the same way. The specimens were aligned vertically straight prior to clamping them onto the Instron machine. The fiberglass samples were loaded in tension until they broke or until the load dropped by 40 percent. The Instron machine recorded all the test data, such as, stress, strain, load, elongation, and ultimate stress. The data for each sample was recorded on the computer then analyzed to obtain the Young's modulus. The Young's modulus was found by graphing the stress against the strain and finding the value of the slope using the computer program Matlab.

\subsubsection{Poisson's Ratio of the Fiberglass Shear Keys}

A series of tests were performed on an Instron 8801 servo-hydraulic testing system to determine Poisson's ratio of the fiberglass test specimens. Tests were run and controlled by the Bluehill 2 computer software which was attached to the Instron machine. Similar to the carbon fiber specimens, strain gages were applied to the lateral direction of the fiberglass specimens. The same technique and materials were used on the fiberglass specimens as previously described in the Poisson's ratio of the carbon fiber composite section. However, for this case the fiber orientation will be changed for each specimen. For the first specimen, the fiberglass strains were placed longitudinally while for the second case the fiberglass strains were placed in a transverse direction. Figure 20 shows the orientation of strain gages that were placed on both types of fiberglass specimens. 


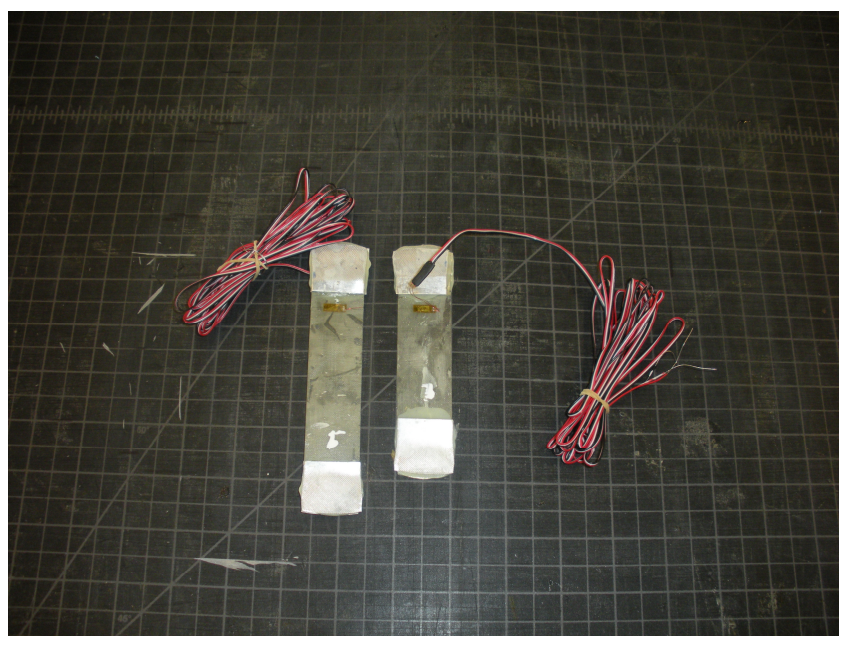

Figure 20: Illustration of strain gage attachment implement of the fiberglass coupon samples.

The Poisson's ratio test was selected in the Instron machine test setup for a tension test that does not fracture the specimen. This test allowed the specimen to experience tension until a maximum load was achieved. Readings from the P-3500 strain box were written manually at specific loadings. When obtaining the Poisson's ratio the strain in the transverse direction was compared to the longitudinal direction at certain loading values. This procedure was done for various samples of both fiberglass cases; a tension test in the direction of the fibers and perpendicular to the fibers. Several steps were taken when testing along the matrix of the composite instead of the fiber direction to prevent fracture of the specimen. Since the tensile strength of the composite was weaker in the direction of the matrix versus in the direction of the fibers, the rate of tension was decreased. The tension rate was decreased by $50 \%$ from .025 inches per minute to .0125 inches per minute to be able to record lower loading values. Along with adjusting the tension rate, the maximum load was also decreased to a lower value of $50 \mathrm{lbs}$.

\subsubsection{Density and Burn Test of the Unidirectional Fiberglass Shear Keys}

The density value of the shear keys was found by taking a sample of the material and performing various volume and mass calculations to find the average density of all the specimens. To determine the density of the Woven Roving Fiberglass, the sample that was already cured was cut to a 1 x 10 inch dimension and re-measured with a micrometer to determine length, width, and thickness of the specimen. The weight was then measured by using the weight scale that was provided by the laboratory. The density was then found by dividing the mass and volume of each sample.

A series of weight tests were done for various fiberglass samples. This testing was done in order to ensure that the fiber content was consistent across several different lay-ups, and that the specimens 
were relatively equal in strength. The burn test was based on a simple 3-step process. First, a 1 x 1 inch specimen was weighed and then placed in a small ceramic oven to separate the resin from the fibers. Once all of the resin was evaporated, all of the fibers that remained were removed from the oven and weighed. The one-inch square specimens were baked in the oven for approximately 45 minutes at $400{ }^{\circ} \mathrm{F}$. Carbon fiber specimens could withstand higher temperatures $\left(\sim 1200^{\circ} \mathrm{F}\right)$ allowing the resin to burn off faster. However, at that temperature fiberglass would burn off, which would remove any possibility of weighing the fibers. Thus, a lower temperature and longer time period was used to ensure that the fibers remained intact for the final weighing.

\subsection{Composite Sandwich Plate Fabrication}

After conducting various experiments to determine the mechanical properties of carbon fiber facesheets and foam core, the sandwich composites were then manufactured and cured inside the autoclave. In this lay-up, two laminas of LTM45/CF1803 prepreg carbon fiber were combined to make a facesheet in which two sets of facesheets were applied on each side of the sandwich plate. The goal of the primary research was to identify the dynamic response of the carbon fiber sandwich composite for different case studies. These cases include control composite plates, delamination regions, shear keys embedded into the foam, and a combination of both shear keys and delamination.

\subsubsection{Cure Cycle and Lay-up of the Composite Sandwich Plate}

The first step of the fabrication method was to cut the foam to the correct dimensions of $12 \times 12$ inches; as previously described. The reason for creating $12 \times 12$ plates was that the release tool surface did not allow for a greater plate area. Pieces were cut in order to have two plates cure during a single curing cycle, but the specimens had a great amount of voids on the surface of the facesheet. Therefore, the composite sandwich plates were manufactured to have an area of $12 \times 12$ inches. After the foam was cut, the carbon fiber was taken from the freezer and cut to $12 \times 12$ inches so that it fit flush with the foam core. The facesheets were then placed on the foam core one at a time to ensure that the orientation of the carbon fiber facesheet was square and in the correct orientation. This part of the process must be handled with care because if a sheet was not squared and with the correct orientation, results between specimens can vary drastically. This process is repeated for both sides of the foam so that there were two sheets of carbon fiber lamina for each side of the foam core. The composite lay-up was similar to the two-layer carbon fiber laminate composite, except that the ideal breather layer was a perforated vacuum bag sheet instead. The results of the breather selection and explanation will later be described in the experimental 
material properties section. The composite sandwich lay-up was then placed into the autoclave and connected to the vacuum pump hoses as seen in Fig. 21.

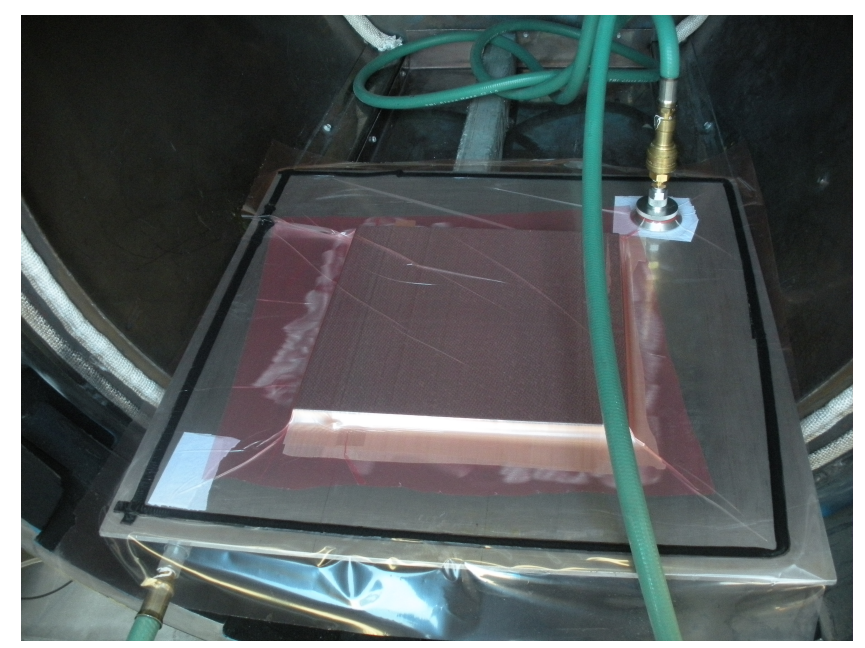

Figure 21: Vacuum pump connections to the complete sandwich composite lay-up.

Similar to the carbon fiber laminate, it was vital to have the vacuum pressure reach at least 25 in. $\mathrm{Hg}$ before the autoclave was turned on. If the vacuum does not reach the specified value of $25 \mathrm{in} . \mathrm{Hg}$, then the bag was checked for leaks or holes within the bag. If the condition of the vacuum pressure was unattainable then the vacuum bag must be removed and a new vacuum bag applied to the lay-up. Once the vacuum pressure reaches $25 \mathrm{in}$. $\mathrm{Hg}$, the composite sandwich plate was left for 30 to 60 minutes to debulk. The autoclave was then turned on and the specimen was removed after the cure cycle had finished.

Inspection is required once the composite has been cured. If there are a minimum amount of voids on the specimen and no discrepancies within the fiber orientation, then the specimen is considered good quality. If the dominant outer layer was not parallel/ perpendicular to the edge of the plate then the specimen was either re-cut or deemed not usable. If the amount of voids was significant on the surface of the carbon fiber facesheet or there were inconsistencies throughout the carbon fiber orientation, the part is put aside for possible lab experiments students can utilize. All cure composite sandwich specimens are left in storage for 10 days until it is cut, in case the composite sandwich specimen had not fully cured completely. Then the specimens are placed inside a cabinet as a precaution in order to have a fully cured plate. The tile saw was used to cut the composite sandwich to a square dimension of $10 \times 10$ inches. The tile saw used for the majority of the cutting can be seen on Fig. 22. Due to the lack of resources this was the only tile saw available for the experiment, so extra precaution was done in order to have a square composite. Since the tile saw releases water when running, the square sandwich composite pieces were left inside a cabinet to dry off before running a test. 


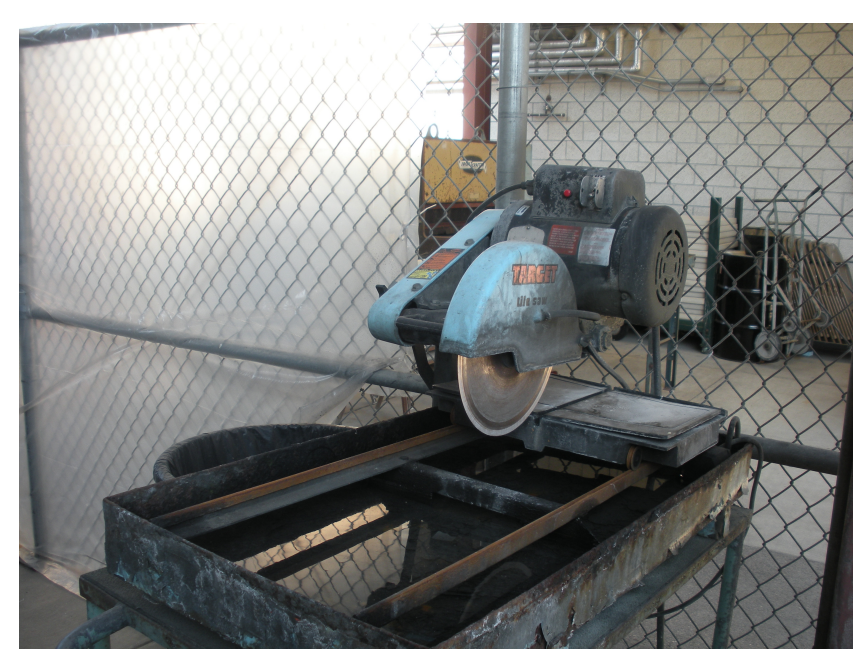

Figure 22: Tile saw used to cut all composite specimens and foam pieces.

To ensure that tight tolerances were made for all specimens, several procedures were done in order to have uniform dimensions for all plates. A straight-edge, ruler, and a white marker were used to assist in making a straight cut. The straight edge ruler was then used for the next cut in order to produce a $90^{\circ}$ angle from the previous cut. The remaining two edges were measured to the exact dimensions and cut on the tile saw. The remaining scraps were discarded due to their size and lack of constant dimension.

\subsubsection{Fabrication of the Delamination Specimens}

The delaminated plate was created the same way as the control plate with the exception that an initial delamination was introduced between the foam core and carbon fiber facesheet interface. The delamination was introduced to the specimen by adding a thin layer of non-porous material, made out of fiberglass, between the facesheets and foam core. The non-porous material does not allow any resin to flow through its boundary, which prevents the two interfaces from bonding together. The delaminated region was constantly made with a 1-inch width and a length between 15 to 18 inches. To ensure that the non-porous material was placed in the correct position, the region of the delamination location was marked on the foam with the use of a color sharpie. The position of the delamination will change in three different locations which include a delamination between 4.5 to 5.5 inches from the base of the $10 \times 10$ inch square plate, delamination occurring from 0.25 to 1.25 inches from the base, and a delamination from 8.75 to 9.75 inches from the base of the plate. Since all the foam cores have a dimension of $12 \times 12$ inches, it is important to note that an extra inch was applied to each delamination placement dimension to ensure when cutting the plate the cut would not affect the location of the delamination. The layers of carbon fiber were added to the foam the same way as the control specimen with two layers of 
LTM45/CF1803 on each side. However, when dealing with delaminated specimens it was recommended to place the carbon fiber facesheet on the foam core side without a delamination first. Doing this would eliminate any chance of altering the location of the non-porous strip if the specimen was flipped. The top surface of the composite was then covered by a porous sheet, a perforated vacuum bag breather, and a vacuum bag in this order. The same procedures were followed as in the control case when placing and connecting the delaminated specimen to the vacuum pump and autoclave.

Once the curing cycle had finished, extra care was taken when cutting the samples to the exact dimensions. For each delamination case, a set of three identical plates was required, which meant that the fiber orientation and the location of the delamination had to match. To ensure this, the carbon fiber laminate was aligned correctly during the lay-up procedure and the specimens had the same cutting process as well. As previously described, the non-porous strips had a length of 15 to 18 inches which specified the locations of the delamination. This allowed accuracy and consistent measurements when marking each set of composite and their desired distances from the delamination. The specimen was cut with the tile saw and a straight ruler and straight edge were used to cut. This process was repeated for the remaining delaminated samples.

\subsubsection{Fabrication of the Composite Sandwich Plate with Shear Keys}

The damage arrestment devices used for this experiment were shear keys, as seen in Fig. 23. These shear keys are the result of the Woven Roving Fiberglass fibers and the Aeropoxy matrix cure, and were embedded into the foam core.

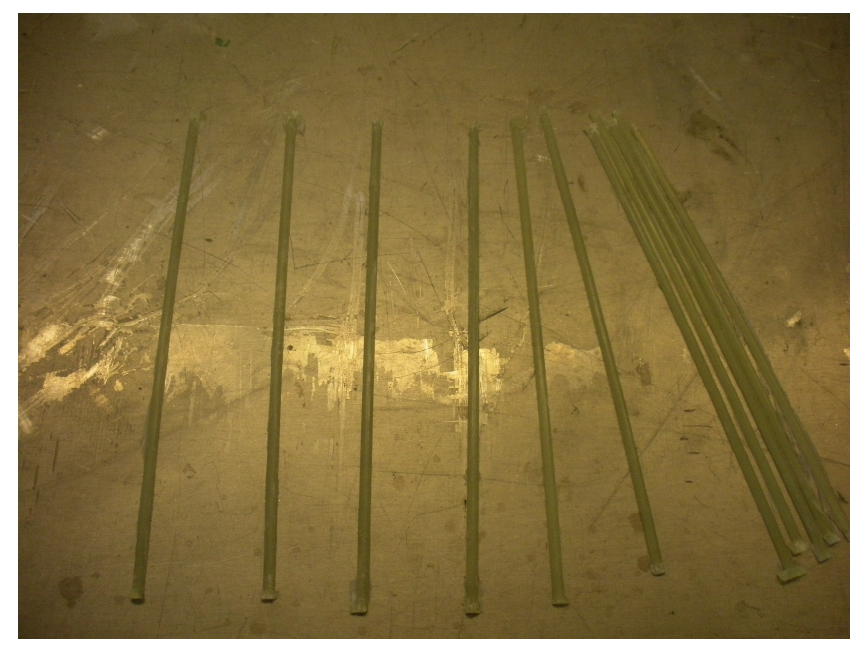

Figure 23: The geometric shape of the cured fiberglass shear keys. 
The foam must be cut precisely in order to have a flat surface when shear keys are inserted into the foam core. If the grooves are cut too deep into the foam the shear keys will not be able to bond to the facesheets; if the grooves are too shallow the shear keys will stick out of the foam and the facesheets will not have a complete bond with the foam core. To ensure accurate semicircle grooves, the foam cores were manufactured on a CNC machine. With the help of the aerospace lab technician, these grooves were cut exactly to the required dimensions.

Grooves were created for the other cases that include top, middle, bottom, and the two vertical cases. In total, 30 plates were cut by the $\mathrm{CNC}$ machine in order to have three specimens for each case. Half of the plates cut on the CNC machine were also used for the control case where shear keys were embedded into the foam, but without a delamination between the core and facesheet. The only difference between the delamination with and without shear keys was the marking on the foam core. By machining grooves into the core, the specified width of the delaminated region was already built into the foam. Therefore, marking the location of the delamination was not necessary for the case with shear keys because they were embedded into the foam core. The same process was followed for the composite layup, curing, and cutting of the specimens as previously described in the delaminated plate section.

\subsection{Composite Sandwich Beam Fabrication}

Composite beams were manufactured to further investigate the effects of shear keys on delaminated specimens under dynamic loading. The dynamic effects on cantilever beams differ from cantilever plates. As a result, to study the full effect of shear keys, beams were also considered for this experiment. To save on cost and time, the composite sandwich beams were not manufactured like the plate samples. Instead, after modal analysis was performed on a plate specimen, the sandwich plate was then re-cut into composite beams.

\subsubsection{Construction of Composite Sandwich Beams}

Unlike the composite plate samples, the amount of specimens tested for each case was increased from three to seven test specimens. When producing composite plates, it became more and more expensive to manufacture a large number of specimens in order to have a reliable average. Just to manufacture one specimen required non-reusable material and wasted water for a period of 16 hours in the autoclave. Fortunately, this was not the case for the composite sandwich beams. The composite beams were created from the composite plates that were already analyzed under dynamic loading. Therefore, acquiring a greater number of specimens was easily obtained for each case study. 
After the composite sandwich plates were vibrated for each case study, the plates were then cut on the tile saw to beam specimens. Knowing that the edges of the composite plate were straight, the beams were created by measuring one inch from the edge. The beams were cut so the longitudinal direction of the beam was parallel to the outer dominant fiber orientation. The plates were cut to ensure only a fraction of the composite beam had a delamination, shear key, or both in the perpendicular direction to the outer layer's fiber direction. Each case consisted of seven beam specimens with a dimension of $1 \times 10$ inches and an average thickness of 0.55 inches. Calipers were used to measure the specimens, which had an exact width of $1 \pm .015$ inches. If this tolerance was not met then the specimen was re-cut or discarded and considered as a non-testable specimen. Figure 24 shows several composite sandwich beams for different case studies.

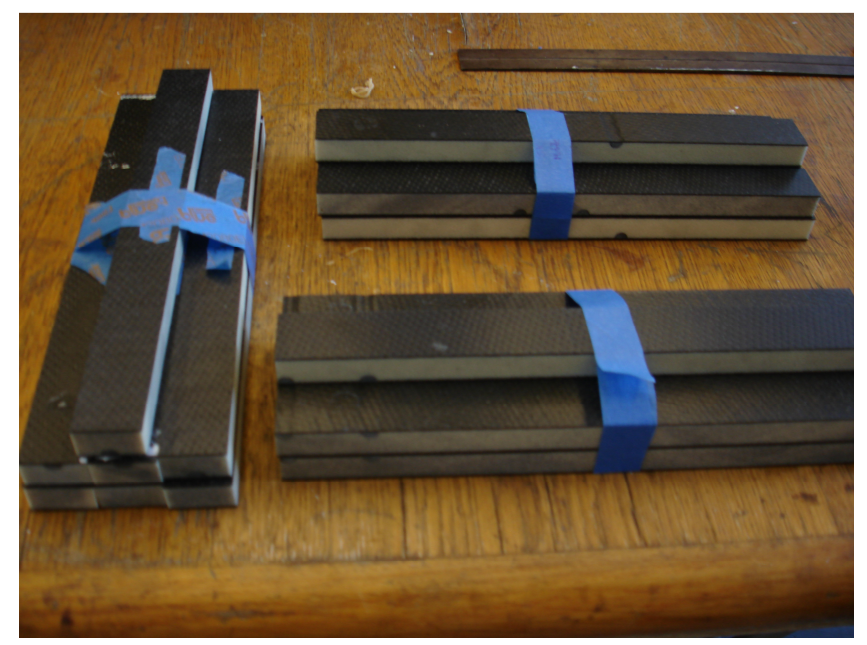

Figure 24: Different cases studies of the carbon fiber sandwich composite beams.

\subsubsection{Fabrication of the Varying Delaminated Composite Sandwich Beam}

From the previous composite beams, shear keys were only inserted in the horizontal direction of the beam. For this iteration one shear key will be placed in the middle and in the vertical direction of each composite beam. The same case studies which include a control, delamination, shear key-only, and delamination with shear key specimens were again studied to determine the effects under dynamic loading. However, only the middle case was studied for this experiment. These beams had to be manufactured unlike the previous composite sandwich beams that were produced from the composite plates. This would require the foam core to have several quarter inch semicircle slots in order to produce various samples for each case. The manufacturing process was identical to the composite sandwich plates for both the lay-up and curing cycle. Once the composite was ready to be cut into beams, the plate were 
aligned so that the shear keys are parallel to the blade. When cutting parallel to the shear keys, it was important to have the shear key in the middle of the beam specimen otherwise the experiment would not have consistent values. Each case consisted of seven beam specimens with a dimension of $1 \times 10$ inches and an average thickness of .55 inches. Calipers were used to measure each specimen, which had an exact width of $1 \pm .15$ inches. If the tolerance was not met then the specimen was discarded or re-cut.

Further experiments were performed to determine how increasing the delamination region would affect the natural frequency for each case study. The beam orientation with a vertical shear key running in the middle of the specimen was used for this experiment. Once again the composite sandwich beams were manufactured to include a delamination region. For this experiment, specimens were manufactured to include a $2 \times 1$-inch or $3 \times 1$ inch delamination region. Once the beams were manufactured they were ready to be vibrated. 


\section{Discussion of Experimental Material Properties}

A series of tests were conducted in order to obtain the material properties for all the specimens throughout the experiment. Knowing the characteristics of each material was crucial in order to conduct a valid comparison between the numerical analysis solution and experimental data for the vibrated composite sandwich specimens. The testing of the material properties includes tensile tests for the carbon fiber and fiberglass along with the compression testing done on the polyvinylchloride foam. For all three materials, testing was done in the transverse and longitudinal directions for each specimen. The volume fraction test results were also considered to determine the consistency of the specimens and their densities through various burn tests.

\subsection{Carbon Fiber Facesheet Material Properties}

The three different methods considered in the lay-up of LTM45/CF1803 prepreg carbon fiber were all tested according to the ASTM D3039 testing standard. The stress versus strain curves for each sample was plotted on a graph that pertained to a certain lay-up method. The types of breathers included

in the study were cotton based, multimedia, and a perforated vacuum bag. However, when the composite samples were cured for the cotton and the multimedia breathers, it was evident they caused a significant amount of voids in the specimens. In contrast, the perforated vacuum bag did not cause substantial voids in the specimens. Therefore, only the perforated vacuum bag and multimedia breather were considered for comparing results. If the results indicated that the multimedia specimens yielded the highest elastic modulus then further investigation would be considered to determine whether the multimedia or the cotton base breather were better.

On each plot, seven samples composed of two layers of carbon fiber were considered. It is important to note that the stress-strain curves slope of the composite is only considering the linear elastic segment when obtaining the elastic modulus of each specimen. The region where the specimen is experiencing buckling then tension at the beginning of each slope is also ignored when obtaining the elastic modulus from the linear region of the curve. The elastic modulus for each test method was then averaged which resulted in an elastic modulus that could be assigned to each method. Figure 25 illustrates the stress and strain curve for each case and depicts the similarity between each test specimen. 


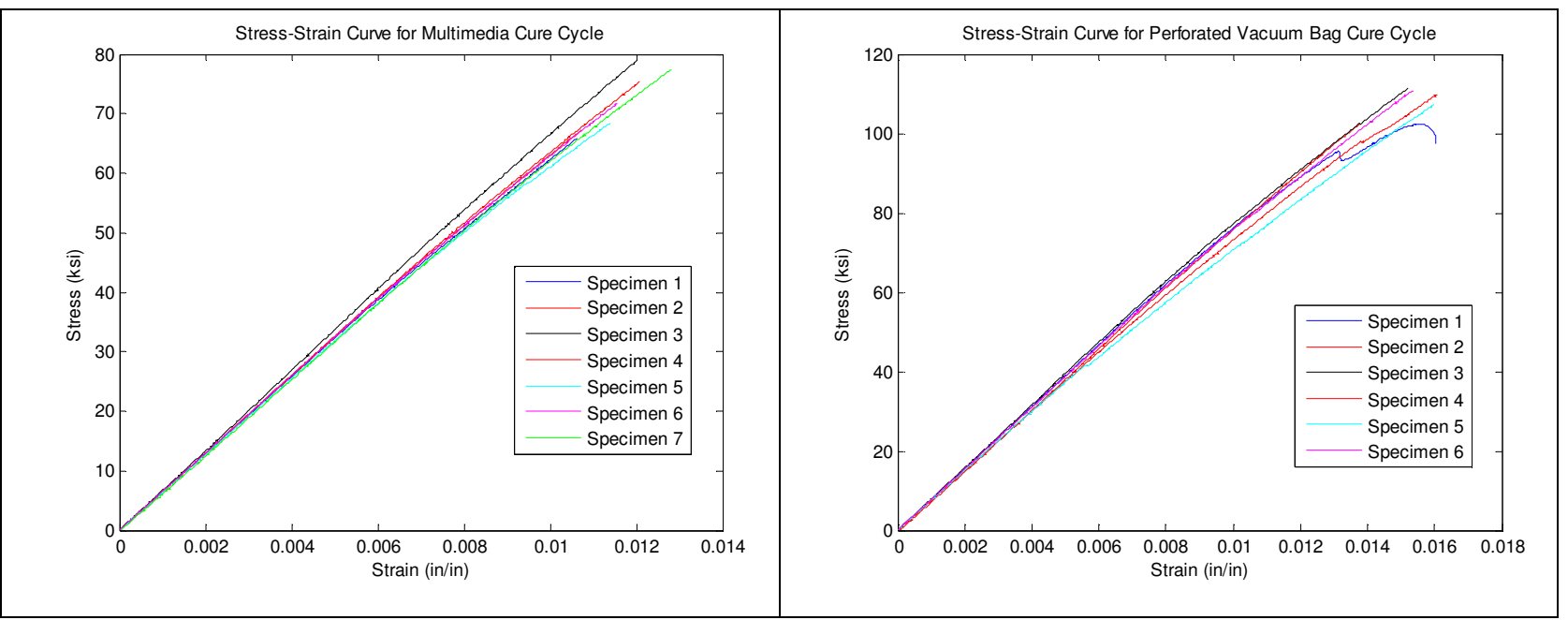

Figure 25: Normal stress and normal strain curve of the carbon fiber facesheet.

Both graphs show consistent trends with the exception of specimen 1 for the perforated vacuum bag cure. There is also a difference in the number of testing samples between the two different lay-ups. The reason for this occurrence is because of the location of where the specimens broke. In the tensile standard, if fracture occurs one inch from the aluminum tabs then the specimen is deemed unusable for the results. Only one specimen broke within the non-tolerable region when using the perforated vacuum bag. The several fractures of the lay-up with the perforated vacuum bag as the breather can be seen in Fig. 26. From the three broken samples, the one on the right was unusable based on the fact that the three fractures occurred one inch from the aluminum tabs.

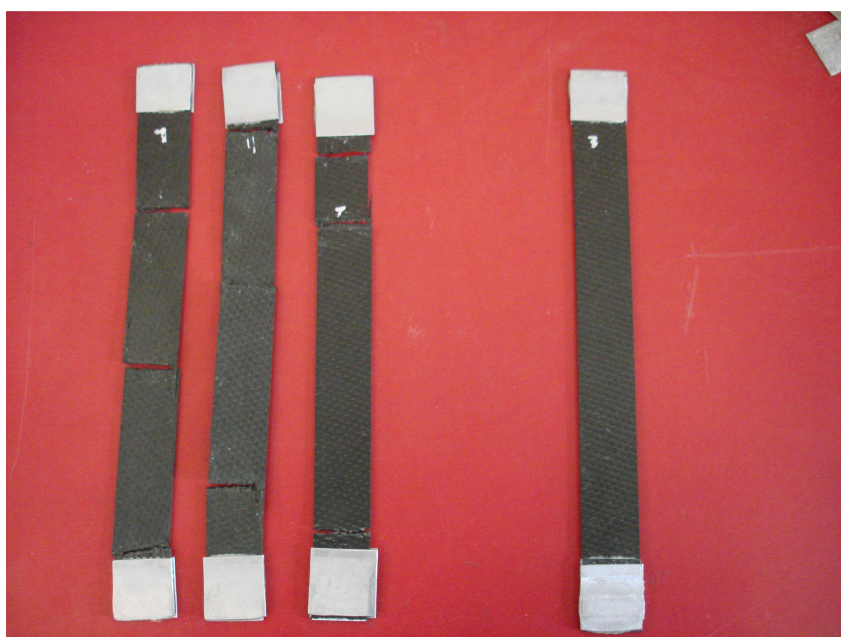

Figure 26: Fracture locations of the carbon fiber specimens after tensile testing. 
The elastic modulus and ultimate stress value for each specimen, that met the tensile standards, were recorded. The ultimate stress value is another important factor when comparing the different carbon fiber samples. When investigating the behavior of the carbon fiber, it is important to verify that the method produces consistent results. Therefore, statistics such as averages and relative standard deviations were calculated for each method. The relative standard deviation of the average ultimate stress and average elastic modulus of each specimen was calculated to verify the results. The relative standard deviation for the perforated vacuum bag case resulted in an elastic modulus of 2.34 percent, which certifies confidence in the values obtained. The ultimate stress had a relative standard deviation of 3.81 percent. The average elastic modulus was 7,772,000 psi and the average ultimate stress was 107,290 psi. The relative standard deviation for the multimedia breather elastic modulus was 2.22 percent with an ultimate stress relative standard deviation of 7.17 percent. The average elastic modulus was determined to be 6,541,000 psi and the average ultimate stress was 72,096 psi. This concluded that the lay-up with the perforated vacuum bag as the breather produced the strongest carbon fiber facesheet for this experiment.

In addition to finding the elastic modulus and ultimate stress of the facesheets, a strain gage was added in the lateral direction and an extensometer strain gage was placed onto the samples to find the Poisson's ratio of the carbon fiber. This is found by taking the negative value of the strain in the lateral direction and dividing it by the strain in the longitudinal direction. However, only the lay-up that included a perforated vacuum bag was considered for this experiment. Each strain was plotted against the force that the specimen experienced which can be seen in Fig. 27. The standard for acquiring a Poisson's ratio also requires that at least five specimens be tested during the experiment.

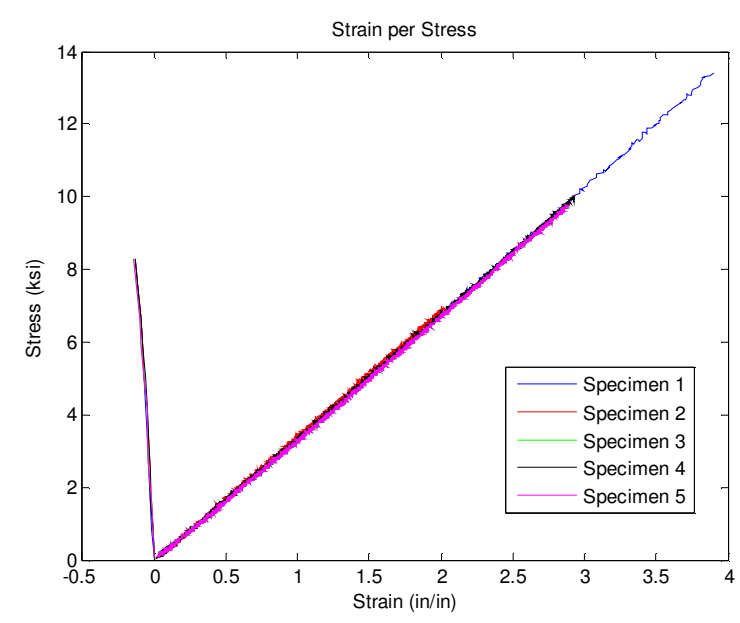

Figure 27: Transverse and longitudinal strain curve relationship of the carbon fiber specimens.

Certain strain values were obtained from the graph at various loading values. The Poisson's ratio was then found by taking the strain in the lateral direction and dividing it by the strain in the longitudinal 
direction. The average Poisson's Ratio for the LTM45/CF1803 prepreg carbon fiber was 0.044, with a relative standard deviation of 1.25 percent. With the Poisson's Ratio known, the Shear Modulus can be calculated by using Eq. 1. The Shear Modulus is a function of the Elastic Modulus E and the Poisson's ratio $v$ of the specimen. However, for the carbon fiber facesheet material, it was assumed that Ey and Ex have the same elastic modulus for both directions.

$$
G x y=\frac{E x}{\left(1-v_{12} * v_{21}\right)}
$$

Facesheet density is relevant to this research because during the finite element analysis, the mass of each element is essential in calculating various mass property values. Experimentally, the values of density are found by taking several specimens and performing various volume and mass calculations to find the density. To find the density of the LTM45/CF1803 carbon fiber facesheets, samples that were already cured were cut to a 1 x 10 inch rectangular specimen where lengths and thicknesses were measured with a micrometer, and the weight was then measured by a scale. The density was then found by dividing the masses of the samples by the volumes of the samples, which came out to be an average of 0.05968 pounds per cubic inch. The same experiment was done for the bad cure cycle that involved multimedia as a breather. The results of this experiment found that the density come 0.046 pounds per cubic inch. This exemplifies why this cure cycle was not the strongest due to the fact that most of the resin was not cured properly with the fiber, thus causing a lower density value.

To validate the experimental density, the theoretical value was calculated using the densities of each constituent along with the volume fraction of the lamina. Burn tests were performed to five specimens from two different cured plates that are shown in Table 1. It is important to note that these results were used to ensure that the layup procedure and techniques used produced consistent results.

Table 1: Burn test results of the $1 \times 1$ inch carbon fiber samples to acquire the weight fraction of the composite.

\begin{tabular}{lccc}
\hline & Weight Before lbs. & Weight After lbs. & Weight Fraction \\
Specimen 1 & 0.0060 & 0.0035 & 58.33 \\
Specimen 2 & 0.0065 & 0.0040 & 61.54 \\
Specimen 3 & 0.0060 & 0.0035 & 58.33 \\
Specimen 4 & 0.0065 & 0.0040 & 61.54 \\
Specimen 5 & 0.0060 & 0.0035 & 58.33 \\
\hline
\end{tabular}

The table shows the weight of the specimen before, the weight of the fibers without resin, and the weight fraction of the fibers. Since the weight and the volume fraction are directly proportional to one another, it was possible to gauge the results by just looking at the weight percentage. The differences 
between the weight fractions for each run showed to be 3.21 percent off from one another. The difference in weight fractions of fibers may have originated from the inaccuracy and limitation of the scale. The density of the LTM45 resin and CF1803 carbon fiber was provided by the Advanced Composites Group Company and was 0.0448 and 0.0636 pounds per cubic inch respectively. With the volume fraction of about 60 percent known, Eq. 2 can be used to find the density of the entire laminate:

$$
\rho_{t}=V_{f} \rho_{f}+V_{m} \rho_{m}
$$

With this equation, the theoretical value of .05608 pounds per cubic inch was found, which differs by only 6.42 percent when compared with the experimental value obtained. From these results of the burn test the conclusion was that the procedure and the techniques used in manufacturing the specimens were consistent and would provide accurate properties for the numerical calculations.

A summary of all the mechanical characteristics testing can be seen in Table 2. This table shows the average elastic modulus, shear modulus, Poisson's Ratio, and density of the two different carbon fiber lay-ups.

Table 2: Mechanical characteristics for the two different carbon fiber lay-ups.

\begin{tabular}{ccc}
\hline & Cotton Breather & Perforated Vacuum Bag \\
& Carbon Fiber & Carbon Fiber \\
Elastic Modulus (psi) & 6541000 & 7772000 \\
Shear Modulus (psi) & $\mathrm{NaN}$ & 3722222 \\
Poisson's Ratio & $\mathrm{NaN}$ & .044 \\
Density (lbs./in3) & 0.0464 & 0.05968 \\
\hline
\end{tabular}

\subsection{Polyvinylchloride Foam Material Properties}

The test method for obtaining the PVC (polyvinylchloride) foam mechanical characteristics follows ASTM D1621 standard. This test standard is the test method for finding compressive properties of rigid cellular plastics. The LAST-A-FOAM FR-6710 was tested perpendicular to the rise of the foam and parallel to the rise of the foam. Two sets containing five samples of $2 \times 2 \times 2$ cubic inch foam specimens were considered for the test. These sets consisted of four segments that were taped together to form the necessary size requirement. It is important to note that the stress-strain curves slope of the LAST-AFOAM FR-6710 is only considered in the linear elastic to obtain the elastic modulus of each specimen. The plastic deformation at the beginning of each slope is also ignored when considering the linear region. The moduli for each test method were then averaged resulting in an elastic modulus that could be 
assigned to each method. Figure 28 illustrates the stress and strain curves for both experiments in addition to depicting how similar each trend line was.

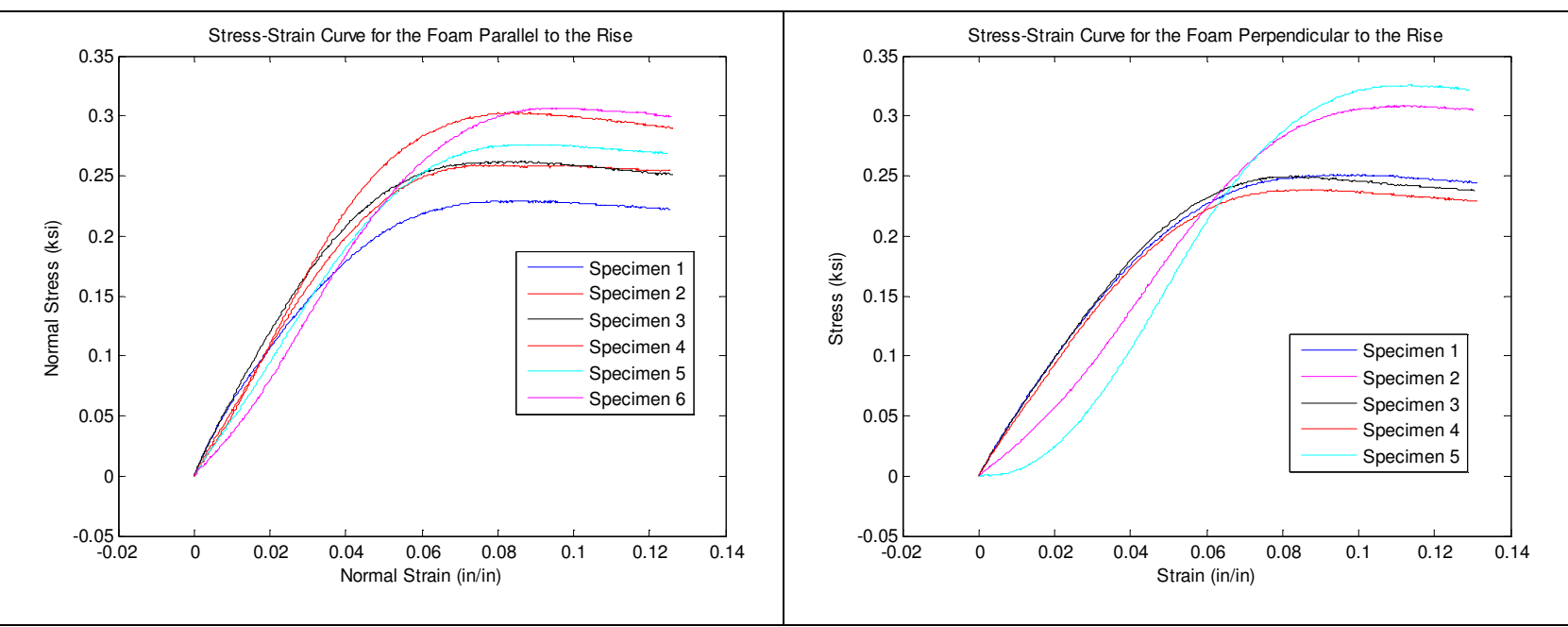

Figure 28: Stress and strain curve for both parallel and perpendicular to the rise of the Last-A-Foam.

Foam density is relevant to this research because during the finite element analysis, the mass of each element is essential in calculating various mass property values. The values of density are found by taking a sample of the material and performing various volume and mass calculations to find the density. To find the density of the LAST-A-FOAM FR-6710, several 12 × 12 inch rectangular plates were measured with a micrometer and ruler to determine the lengths, widths, and thickness for each specimen. The weight was then measured using a scale provided by the Cal Poly Composites Lab. The scale used was the aeAdam scale which had a precision of ten thousandth when measuring in lbs. The density was then found by dividing the masses of the samples by the volumes of the samples, which came out to be an average of 0.0059 pounds per cubic inch.

Having access to the manufacturer's material properties, LAST-A-FOAM FR-6710 was the only material for which the experimental and manufacturer's material properties could be compared. Table 3 shows this comparison along with the various material properties. At first glance, it is evident that there is a significant difference between the experimental values and the values that the manufacturers have given to the public for their item.

Table 3: Comparison between the manufacture and experimental mechanical characteristics for Last-AFoam FR-6710

\begin{tabular}{lcc}
\hline & Manufactured & Experimental \\
Elastic Modulus xy (psi) & 19665 & 5074 \\
Elastic Modulus xz (psi) & 10052 & 4414.6 \\
\hline
\end{tabular}




\begin{tabular}{lcc}
\hline Shear Modulus xy (psi) & 3195 & 1951.5 \\
Shear Modulus xz (psi) & 2823 & 1697.3 \\
Poisson's Ratio & 0.3 & 0.3 \\
Density (lbs./in3) & .00578 & .0059 \\
\hline
\end{tabular}

There are various reasons for this discrepancy between the two value sets. One reason is that the Cal Poly Composites Lab did not have the adequate specimen dimensions for this standard. The largest test specimens to run this experiment were $2 \times 2 \times .5$ cubic inches, which was not acceptable for this ASTM standard. When combining four specimens to make one acceptable piece, it could have easily altered the strength of the material and given an invalid result. The other reason for this disagreement is the fact that the foam material was left outside of the lab where the climate in San Luis Obispo could degrade the material strength of the foam. Therefore, the material properties were reevaluated during the vibration process of the experiment.

\subsection{Fiberglass Material Properties}

The Woven Roving Fiberglass with Aeropoxy resin composite coupons were all tested according to ASTM D3039 testing standard, and the results were all compared. The stress versus strain curves for each sample was plotted on a graph that pertained to a certain orientation of the fibers. On each plot, five samples of a single layer of fiberglass composite were considered. The moduli for each test method were then averaged resulting in an elastic modulus that could be assigned to each method. Figure 29 illustrates the stress and strain curve for each case in addition to showing how the specimens compare to each other.

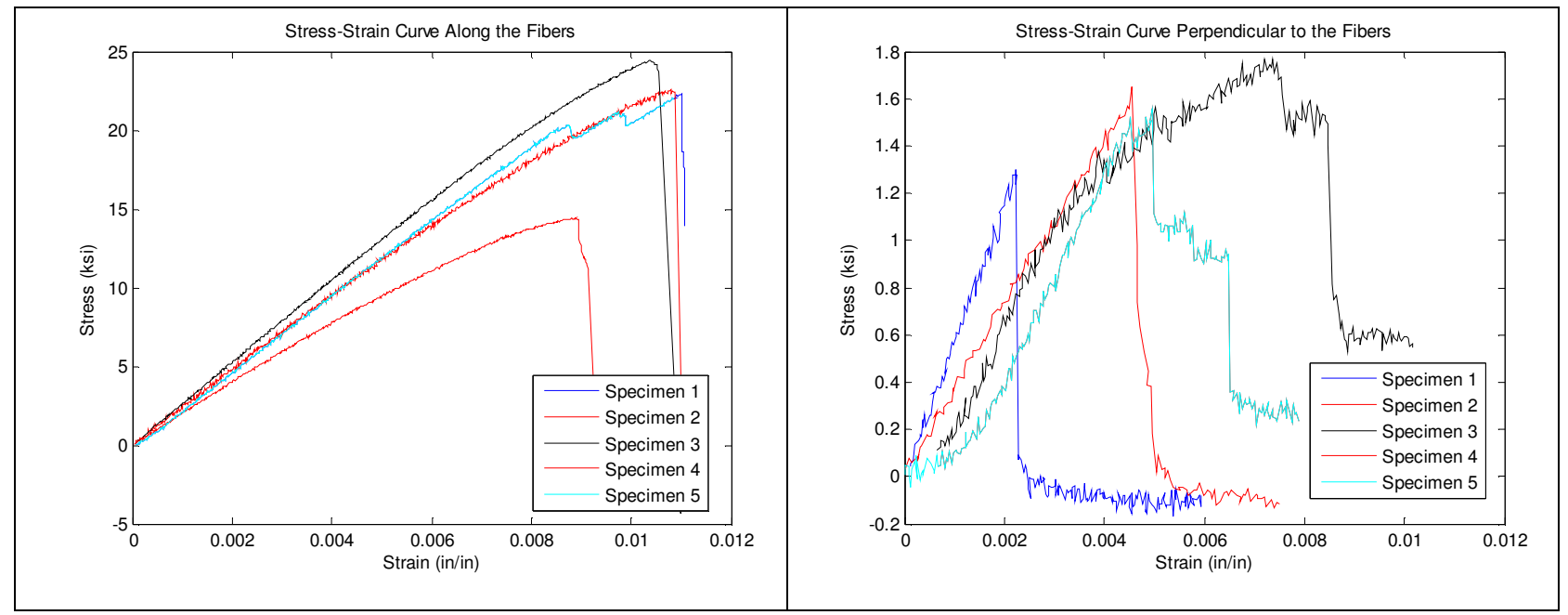

Figure 29: Normal Stress and Normal strain curves for both fiber directions of the fiberglass samples. 
Both graphs show relatively consistent slopes but have varying maximum stress and strain values. Both graphs also show different trends in terms of the appearance of the line. For the stress and strain plot when tension is applied parallel along the fibers, the lines are smooth unlike when the tension is applied perpendicular to the fibers where the lines are jagged. The reason for this occurrence is the loading condition that each case is experiencing. In the case when tension is applied parallel to the fibers, the maximum force is around $425 \mathrm{lbs}$. which is about 10 times higher than the other loading case. With the axis of the stress being compressed to lower values, the noise associated with pulling the specimen is more likely seen. Nonetheless, both stress and strain curves are valid to obtain the elastic modulus for each type of orientation.

In addition to recording all the elastic moduli for each orientation type, the ultimate stress values were also recorded for each sample. The need for a method that produces consistent results is of great importance when investigating sandwich beam behavior; for this reason, statistics such as averages and relative standard deviations were considered for each method. Relative standard deviations were calculated for both the elastic modulus and the ultimate stress to ensure that the results were valid. The relative standard deviation for the elastic modulus parallel to the fibers was 3.23 percent, which conveys statistical confidence in the values obtained. The ultimate stress had a relative standard deviation of 2.81 percent. The average elastic modulus was 2,378,463 psi and the average ultimate stress was 21,222 psi. The relative standard deviation for the elastic modulus perpendicular to the fibers was 4.21 percent with an ultimate stress relative standard deviation of 6.24 percent. The average elastic modulus was determined to be 455,862 psi and the average ultimate stress was 1,566 psi.

In addition to finding the elastic modulus and ultimate stress of the fiberglass composites, a strain gage was added in the lateral direction and an extensometer strain gage was attached to the specimen in order to find the Poisson's ratio of the material. This is found by taking the negative of the strain in the lateral direction and dividing it by the strain in the longitudinal direction. Unlike the carbon fiber coupons which are woven and bidirectional fibers, the fiberglass samples are uni-directional. This meant that both types of fiberglass orientations were tested to acquire accurate results. Each strain was plotted versus the force that the specimen experienced, which can be seen in Fig. 30. 


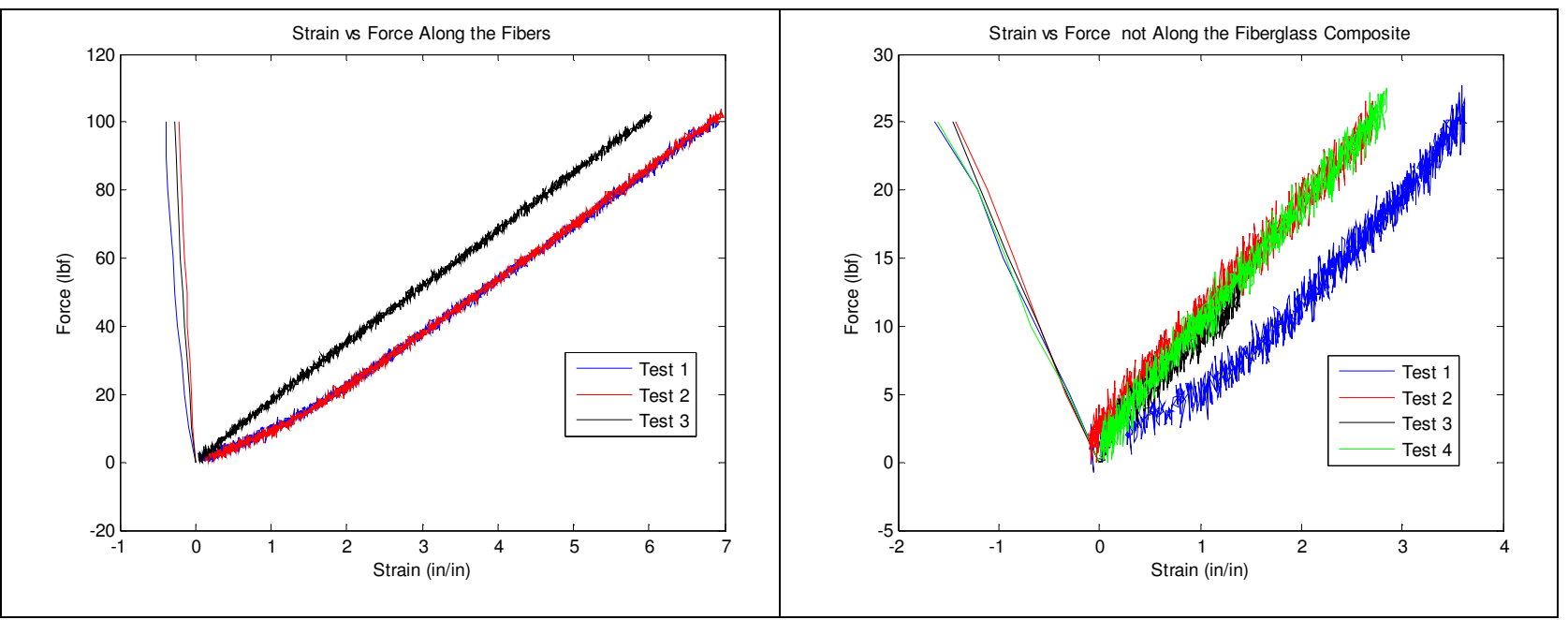

Figure 30: Transverse and longitudinal strain curve relationship for both fiber directions of the fiberglass samples.

Certain strain values were obtained from the graph at various loading values. The Poisson's Ratio was then found by taking the strain in the lateral direction and dividing it by the strain in the longitudinal direction. The average Poisson's Ratio for the Woven Roving Fiberglass with Aeropoxy resin was 0.05 and 0.41 for when tension was applied to the fibers parallel and perpendicular, respectively. Along with finding the Elastic Modulus and Poisson's ratio for the fiberglass shear keys, the density of this material is extremely important in conducting numerical nodal analysis.

Experimentally the values of density are found by taking several specimens and performing various volume and mass calculations to find the density. To find the density of the Woven Roving Fiberglass with Aeropoxy resin, samples that were already cured were cut to a 1 x 10 inch rectangular specimen where lengths and thicknesses were measured with a micrometer and the weight was then measured on a scale. The density was then found by dividing the masses of the samples by the volumes of the samples, which came out to be an average of 0.0581 pounds per cubic inch.

To validate the experimental density, the theoretical value was calculated using the densities of each constituent along with the volume fraction of the lamina. Burn tests were performed to five specimens from two different cured plates that are shown in Table 4. It is important to note that these results were used to ensure that the layup procedure and techniques used produced consistent results.

Table 4: Burn test results of the $1 \times 1$ inch fiberglass samples to acquire the weight fraction of the composite.

\begin{tabular}{cccc}
\hline & Weight Before lbs. & Weight After lbs. & Weight Fraction \\
Specimen 1 & 0.0040 & 0.0015 & 37.50 \\
Specimen 2 & 0.0040 & 0.0020 & 50.00 \\
Specimen 3 & 0.0040 & 0.0020 & 50.00 \\
Specimen 4 & 0.0040 & 0.0020 & 50.00 \\
\hline
\end{tabular}


The table shows the weight of the specimen before, the weight of the fibers without resin, and the weight fraction of the fibers. Since the weight and the volume fraction are directly proportional to one another, it was possible to gauge the results by just looking at the weight percentage. The relative standard deviation for the weight fraction for the five fiberglass specimens was 11.09 percent. The difference in weight fractions of fibers may have originated from the inaccuracy and/or the accuracy of the scale. The density of the Woven Roving Fiberglass fibers and Aeropoxy resin was 0.0533 and 0.0657 pounds per cubic inch, respectively. Unlike the LTM45/CF1803 prepreg carbon fiber, these values were not given by the provider and were found experimentally. With the volume fraction of about 46 percent known, the theoretical value of .05999 pounds per cubic inch was found, which differs by only 3.15 percent when compared with the experimental value obtained. From these results of the burn test, the conclusion was that the procedure and the techniques used in manufacturing the specimens were consistent and would provide accurate properties for the numerical calculations.

A summary of all the mechanical characteristics testing can be seen in Table 5. This table shows the average elastic modulus, shear modulus, Poisson's Ratio, and density of the two different fiberglass fiber orientations.

Table 5: Woven Roving Fiberglass with Aeropoxy resin composite material properties.

\begin{tabular}{lc}
\hline Material Property & Woven Roving Fiberglass \\
Elastic Modulus xy (psi) & 2378463 \\
Elastic Modulus xz (psi) & 455862 \\
Shear Modulus xy (psi) & 113260 \\
Shear Modulus xz (psi) & 146109 \\
Poisson's Ratio xy & 0.49 \\
Poisson's Ratio xz & 0.41 \\
Density (lbs./in3) & 0.0581 \\
\hline
\end{tabular}




\section{Dynamic Response of the Composite Sandwich Specimens}

The primary goal of this research was to determine the effect of damage arrestment devices on vibration response. Previously the damage arrestment devices, shear keys, were used to delay the delamination process in terms or shear and bending force. This study focuses on determining the effects of shear keys under dynamic loading. Two geometry cases were conducted to understand whether shear keys help increase the natural vibration frequency of a composite when delaminated and if shear keys decrease the vibration amplitude of the composite. As previously described, each geometry set contains a control, delaminate, shear keys only, and delamination with shear keys specimens. The location of the delamination and shear key also changed for each case study. For the composite sandwich plate, five different locations were examined throughout the experiment. For the composite sandwich beam case, a total of three different locations were observed. Along with finding the natural vibration frequency and the vibration amplitude of each specimen, certain cases were more close examined to find the effects of shear keys in the time domain.

\subsection{Experimental Setup and Procedures}

In this experiment, the natural frequencies were obtained using the Cal Poly shake table. The composite sandwich plates and beams were secured onto the horizontal position of the shaker table between two aluminum solid blocks. These aluminum blocks were then screwed onto the table and flushed against the composite specimen. With the use of a torque wrench, the screws were consistently tightened to the specific value of $16 \mathrm{ft}-\mathrm{lbs}$. onto the magnesium vibration table. Two accelerometers were placed to record data from the vibration table. The control accelerometer was placed on one of the aluminum block surfaces, while the other accelerometer was placed on the composite sandwich, as seen in Fig.31. Note that the accelerometers must be placed parallel to the surface of the horizontal shaker table. The two accelerometers were then connected to the main computer through two channel ports with the first port being for the control and the second for the specimen's accelerometer. An illustration of the set-up is also depicted in Fig.31 showing the connections, shake table, and computer used for the entire experiment. 


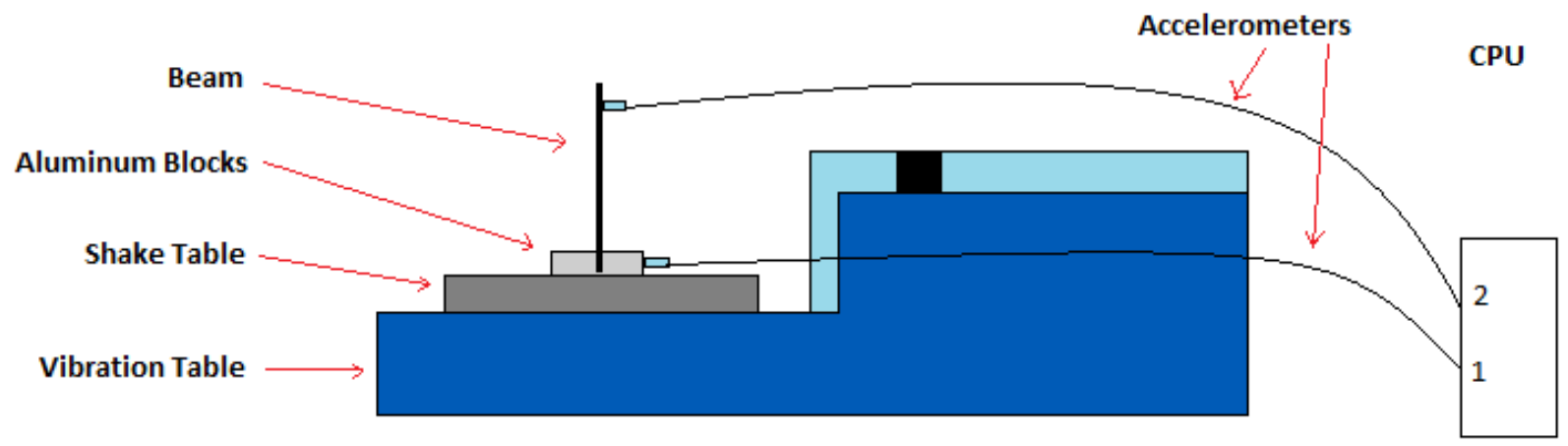

Figure 31: Set-up for the composite sandwich specimens to undergo vibration test.

In order to run the experiment, the vibration table was turned on to heat the oil that was located under the vibration table. Once all connections from the shake table to the computer are made, the frequency software was loaded and run. The program used for this experiment was WinII which allows the user to input a range of frequencies and place an initial acceleration onto the accelerometers. For this experiment, the frequency ranged from $5 \mathrm{~Hz}$ to $1500 \mathrm{~Hz}$ in a sinusoidal sweep. The control accelerometer was set at different g levels which can be seen later on during this chapter. After setting the correct criteria, the gain was turned up to its maximum, the two power buttons were pushed, and the experiment commenced. The program calculated the frequency response of each type of specimen and plotted the $g$ force experienced over the frequency range.

One important aspect that was unforeseen in the beginning of the experiment was the location of the accelerometer. Even though the weight of the accelerometer was only $0.017 \mathrm{lbs}$, it would change the natural frequency that was being recorded onto the WinII software. For this reason, the location of the accelerometer was varied on the composite plates in order to understand how the natural frequency changed depending on the location of the accelerometer. For the composite beam, the accelerometer was placed on the top ends of the specimens to not interfere with the amplitude of the specimen. Figure 32 shows an example of where the accelerometer was placed in both the plate and beam composite sandwich. 

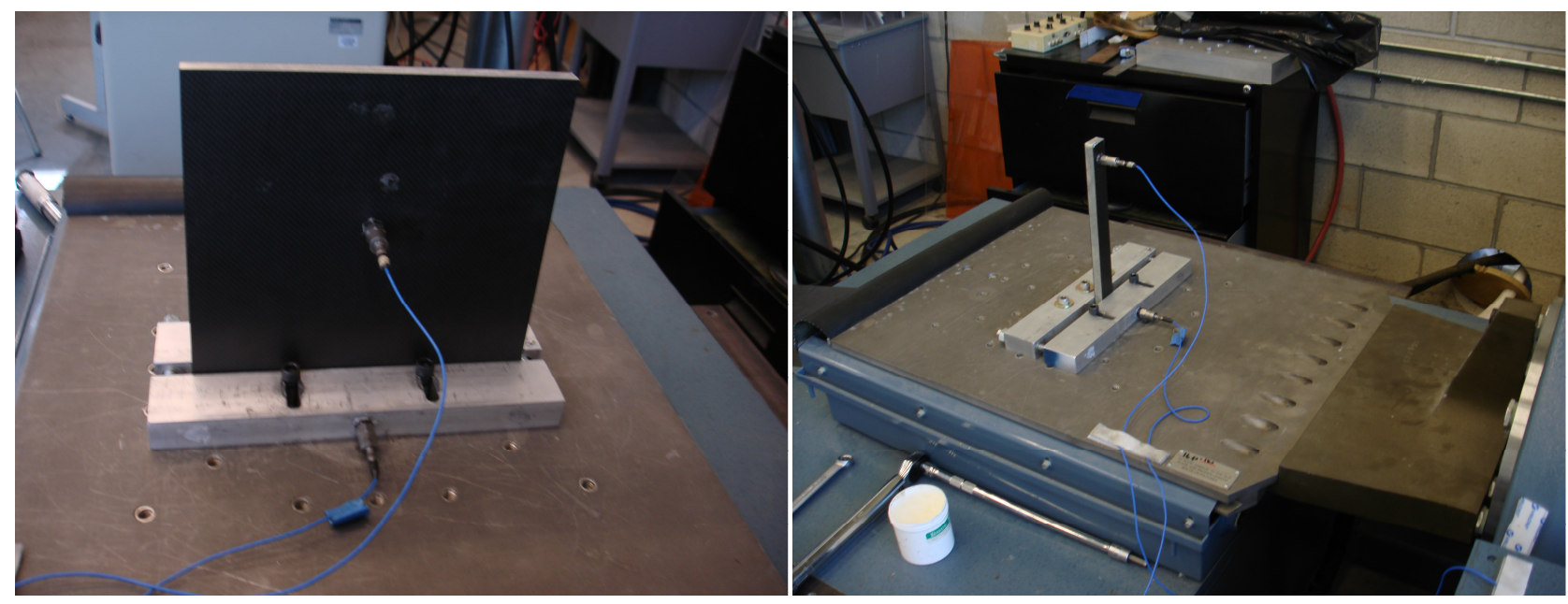

Figure 32: Placement of the accelerometer for both the plate and beam experiments.

\subsection{Dynamic Response of the Composite Sandwich Plates}

To determine the full effects of the damage arrestment devices, several configurations were needed which included: control specimens, delaminated specimens, shear keys only specimens, and a combination of shear keys and delamination. These plates were then placed onto the vibration table to undergo nodal analysis. One important aspect of constraining each specimen onto the table was how the geometry would be altered. If we revisit Fig. 32, it shows that a portion of the plates is being compressed by the two aluminum blocks. The one inch aluminum blocks would only permit $90 \%$ of the actual composite plate to be free to vibrate. Even though the plate is $10 \times 10$ inches in area, only the 9 x 10 inch section of the plate would be allowed to experience the vibration effects.

Along with having reduced plate geometry, the accelerometer location, as stated previously, would be changing throughout the entire experiment on the composite plate. Three specific locations were used for each case study to determine how the natural frequency would change. The first accelerometer position was directly in the middle of the composite sandwich plate at a location of 5 inches from the top surface of the magnesium table or 4 inches from the top of the aluminum block that compressed a portion of the specimen. The second modification was moving the accelerometer to a location off center and above the previous location. The location ended up being 2.5 inches to the right of the center of the plate and at a location of 7 inches from the top surface of the magnesium table or 6 inches from the top surface of the aluminum block. The final position of the accelerometer was an inch from the top of the specimen.

Along with having different configurations for the composite sandwich plates and beams, the location of both the shear key and delamination were also changed. For the composite sandwich plate case, five different case studies were performed to determine how the location of the delamination affected the frequency response of the composite specimen. These locations included shear keys and/or 
delamination occurring horizontally on top, bottom, and middle of the composite sandwich plate. Along with the horizontal positions, vertical locations occurring in the center and side of the composite beam were also considered in this research. An illustration of these orientations can be seen in Fig.33.

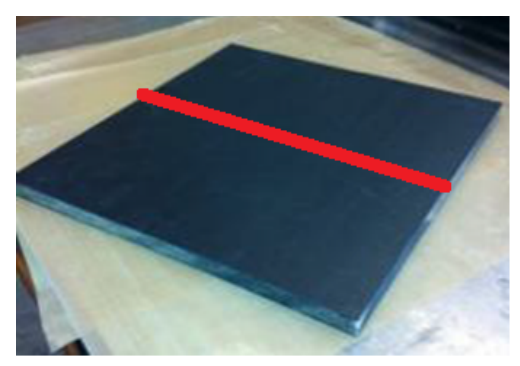

Middle Delamination

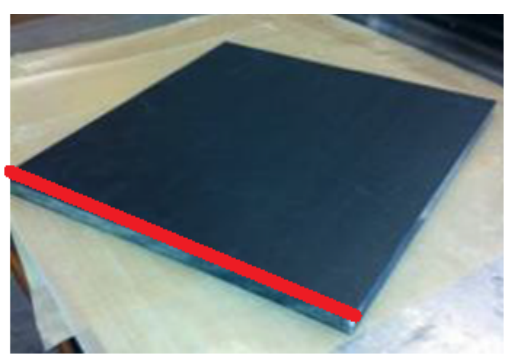

Bottom Delamination

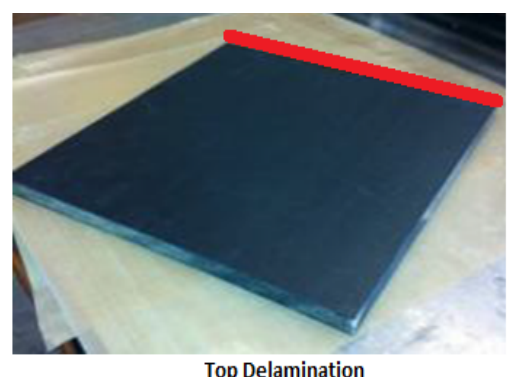

Top Delamination

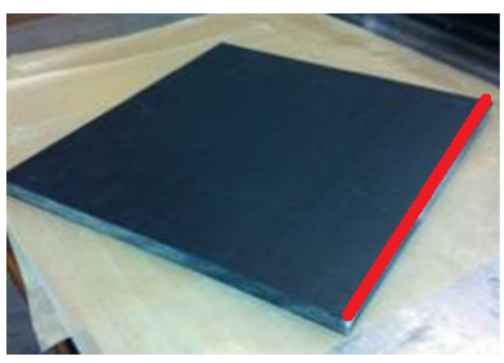

Side Delamination

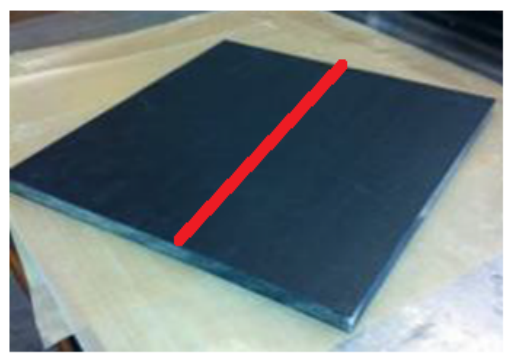

Vertical Delamination

Figure 33: Location of the shear key and/or delamination for the composite sandwich plates.

\subsubsection{Modal Response of the Control Specimens}

A total of three different types of composite sandwich plates were studied for the control case. The experimental data obtained from the Winll software were then transferred to the software program Matlab to be assimilated for analysis. Figure 34 shows the frequency response of specimen 1 for the different accelerometer locations. The location of the accelerometer was first placed 5 inches from the base of the plate and 5 inches from the left edge of the plate. The second location is 2.5 inches from the right of the center of the plate and 7 inches from the bottom of the plate. The last accelerometer location was placed in the middle of the specimen and at a height of 9 inches from the bottom of the composite plate. On the illustration below, the second accelerometer location is labeled as $(2.5,6)$, in which the value 6 denotes the distance of 6 inches from the top of the aluminum block. 


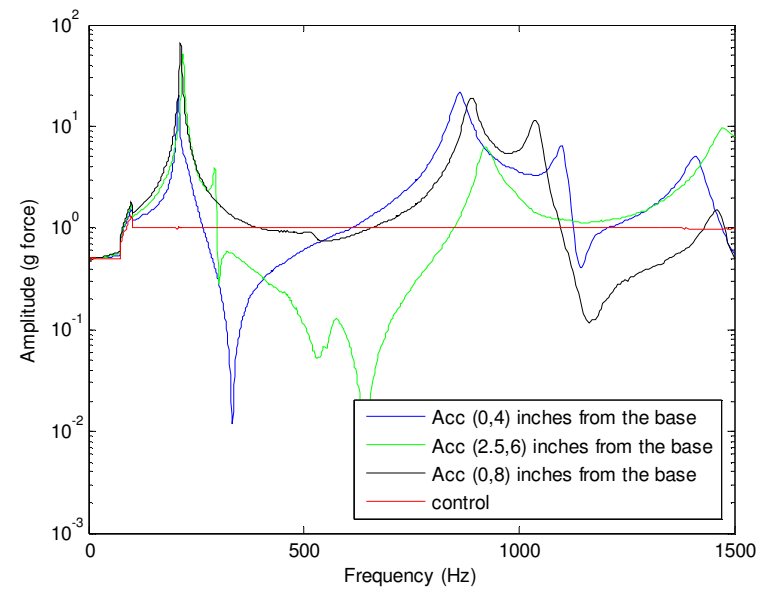

Figure 34: Frequency response for the control specimen 1 for various accelerometer locations.

Figure 35 shows that the location of the accelerometer is very important in terms of altering the natural frequency and amplitude of the specimen. Adding mass to the end of a plate structure typically increases the amplitude and resonance frequency. When the mass is shifted to one side of the structure, the natural frequency is shifted to the right as well. The amplitude is also decreased when compared to the other two accelerometer locations which reside in the center of the specimen. When comparing these results with the finite element models, it shows that when the accelerometer is closer to the middle of the structure, it will have more accurate results when comparing the numerical and experimental values. Therefore, further analysis was done and compared while the accelerometer was placed directly in the middle of the composite plate. Figure 35 shows the results of all three control sandwich plates with the accelerometer in the middle of the structure. This figure shows that the first natural frequency and the third natural frequency are very similar to one another as is the amplitude for all the modes. However, the third and fourth natural frequency has slight differences in value when comparing each specimen. The relative standard deviations for the second and fourth modes, respectively, were 2.63 and 1.39 percent, which confirms that the values were similar to each other. 


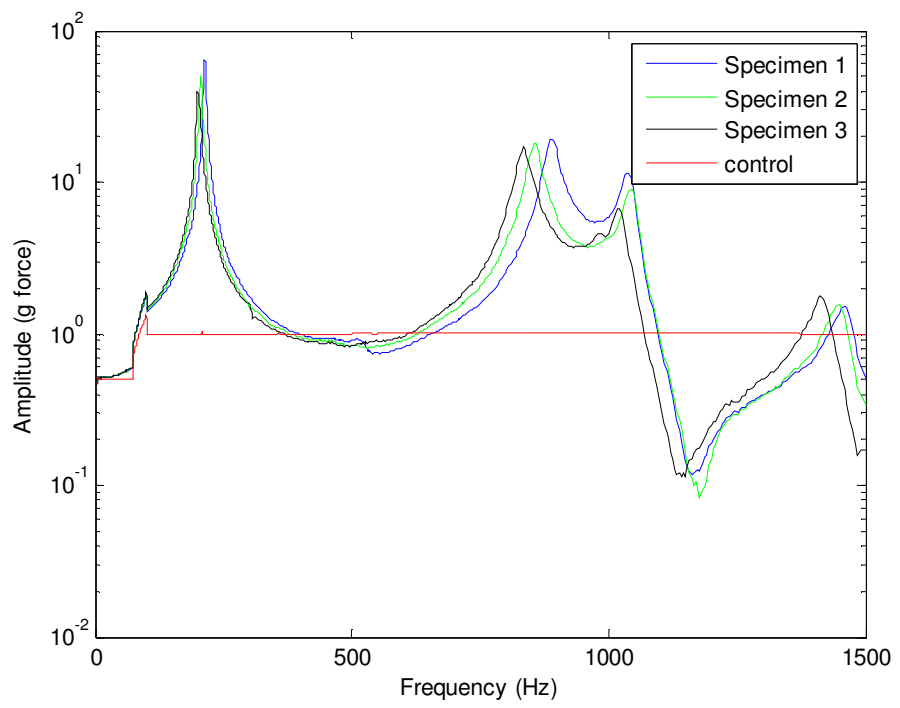

Figure 35: Frequency response for all three control plate specimens for when the accelerometer is placed in the middle of each specimen.

By taking a closer look at the data for both the frequency and amplitude for each mode of the composite plate, it is evident that the three specimens were similar in all aspects. The only exception is the amplitude of the first mode where the magnitude ranges from $40 \mathrm{~g}$ 's to $65 \mathrm{~g}$ 's for the control composite plate. However, the trends were alike with the highest amplitude occurring in the first mode and the lowest occurring in the fourth mode, which are seen in Fig.36. From this data it can be concluded that the control specimens are performing similarly to one another.
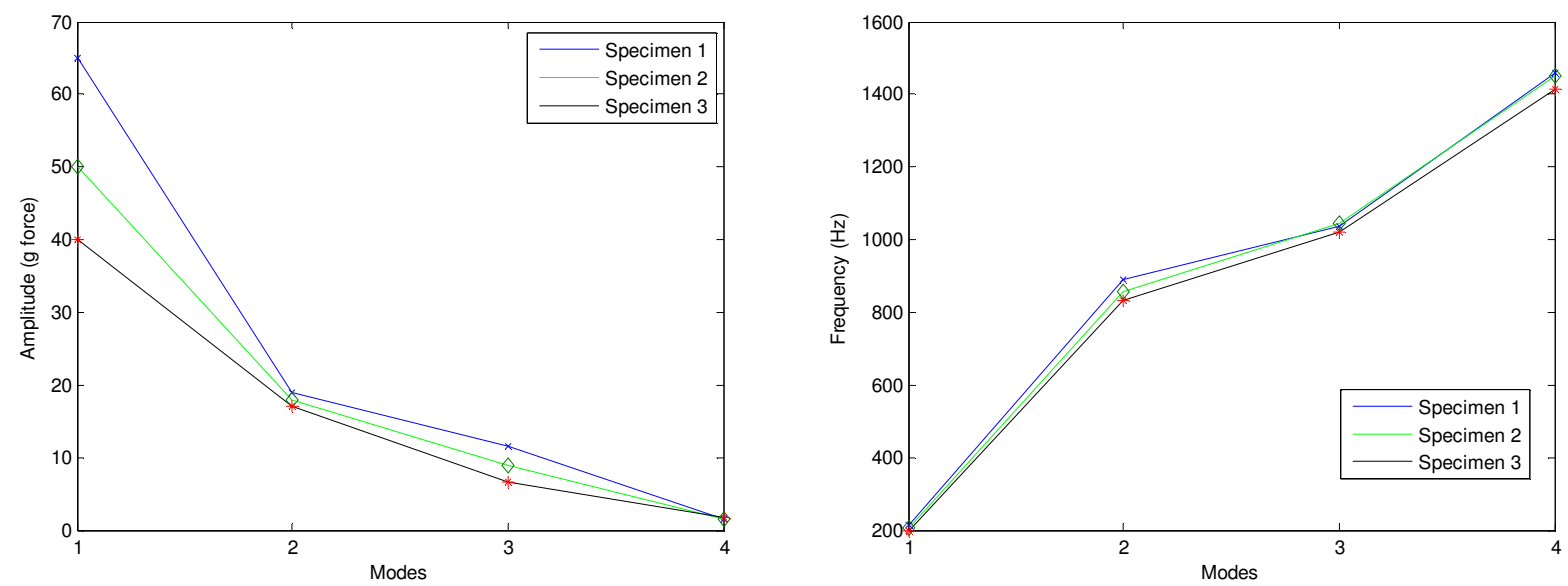

Figure 36: Amplitude and frequency response for all control specimens. 


\subsubsection{Modal Response of the Delaminated Specimens}

In order to fully understand how delamination affects the vibration properties of a composite plate, different orientations of the delamination region were tested. However, instead of depicting each graph and the frequency characteristics for each sample in each sub-case, only one set will be fully described in detail unless there was an anomaly or significant change between the sub-cases. For this case, the middle delamination will be further evaluated to understand the changes in frequency and amplitude a delamination causes to the structure. Figure 37 shows the frequency response of the middle delaminated specimen in response to the accelerometer change. This illustration once again confirms that the accelerometer located off center of the specimen will shift the frequency to the right. The accelerometer placed on the top also causes the amplitude to have the highest value in the first mode in comparison to the other two locations.

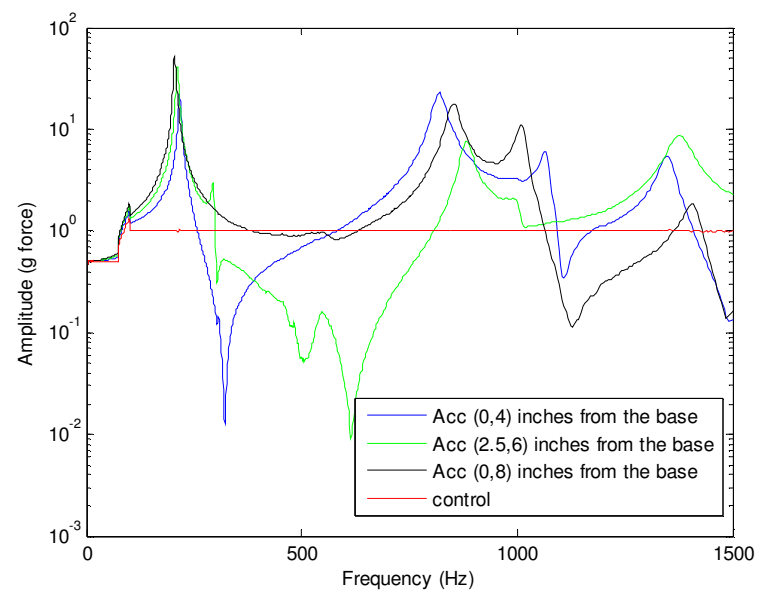

Figure 37: Frequency response for the middle delaminated specimen 1 for various accelerometer locations.

When further investigating the frequency response for each specimen when the accelerometer was placed in the center of the specimen, it is clear that there was an outlier in the results. Figure 38 shows the frequency response for all three middle delaminated specimens with the accelerometer location placed in the center of the plate. While two of the specimens had nearly identical responses, the last specimen was completely different for the first mode. To understand why this phenomenon had occurred, the test was rerun, and the results came out to be the same. It wasn't until the plates were measured once again that it was discovered why this dramatic change had occurred. The size of the plate was 0.25 inches smaller in length than the other two pieces. With a different size than the other samples, it is clear why the first natural frequency behaved differently than the other two specimens' natural frequency. 


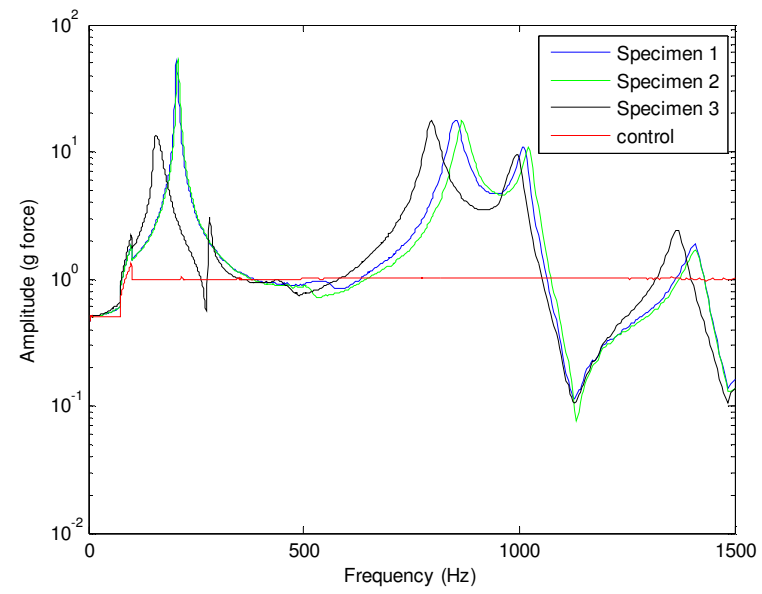

Figure 38: Frequency response for all three middle delaminated plate specimens for when the accelerometer is placed in the middle of each specimen.

Figure 39 takes a closer look at the amplitude and frequency at each mode. In both plates, the third sample behaves differently than the other specimens in both frequency and amplitude. There is a significant difference between the amplitude in the first mode and in the rest of the modes. This could easily have been because of the height difference or because of the orientation of the carbon fiber laminates. Nonetheless, when averaging the specimens to compare with other results, the third specimen was taken out in order to prevent distorting the correct response of the middle delaminated plate.
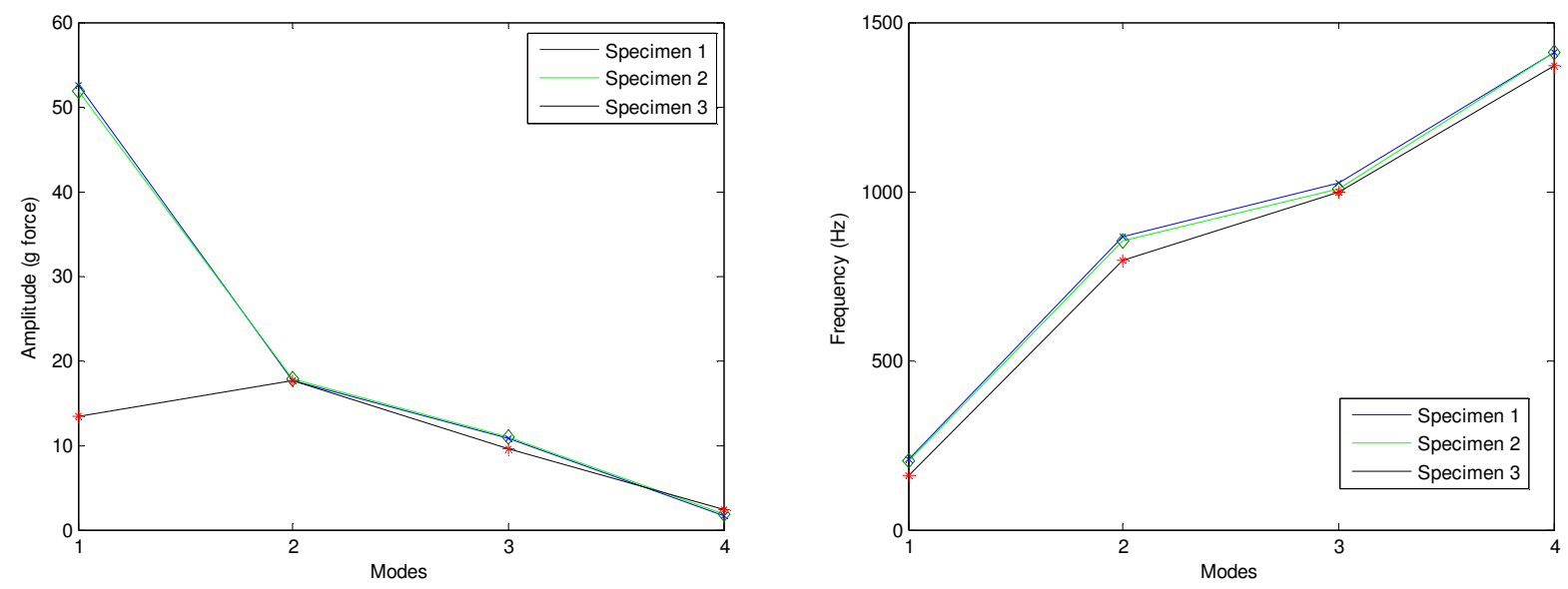

Figure 39: Amplitude and frequency response for all middle delaminated specimens.

The remaining specimens for the delaminated case study had similarities throughout each subcase. Figure 40 shows the amplitude and frequency results of the bottom delaminated case at each mode. This trend was similar in terms of having all three specimens behaving in the same fashion. Along with having similar specimens, the data results for all the sub-cases have the same trend in which the amplitude 
decreases for every natural frequency.
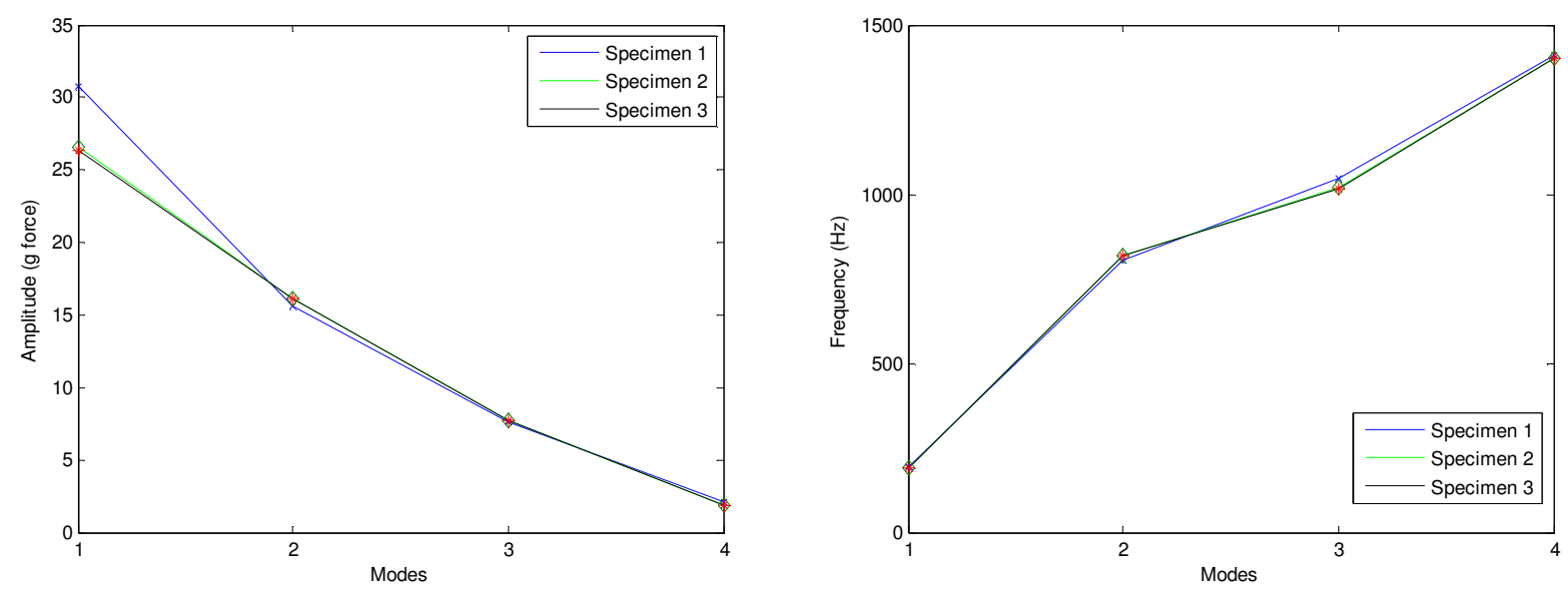

Figure 40: Amplitude and frequency response for all bottom delaminated specimens.

\subsubsection{Modal Response of the Shear Keys Specimens}

The model response of the specimen with shear keys without any delamination between the foam core and carbon fiber facesheet was important to have to compare with the control specimens. The first noticeable feature of embedding a shear key inside the foam core was the change in amplitude when the accelerometer was placed away from the center of the plate. In the previous examples, the frequency response was shifted over to the right to compensate for the accelerometer location. However, Fig. 41 shows that a plate with two shear keys in the middle of the composite plate also shifted the amplitude of the composite plate when the accelerometer was placed away from the center. There was also a missing third mode for when the accelerometer was placed 2.5 inches from the center and 7 inches from the bottom of the plate. In every case the third mode was very small in terms of amplitude, and for the middle, case the mode did not exist. This would suggest that applying shear keys into the foam could have prevented the third resonance from occurring when mass is applied to the corner edge of a composite plate. However, this could not be determined from this research, but could be further investigated in future research. 


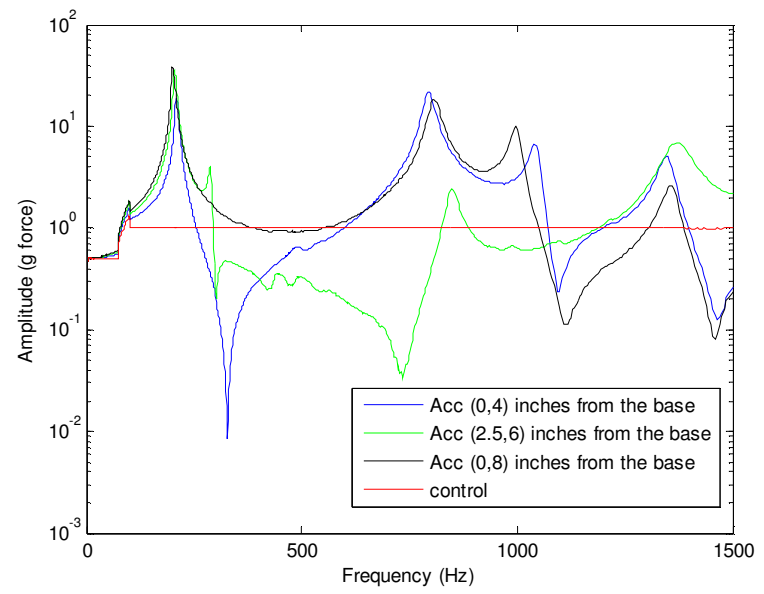

Figure 41: Frequency response for specimen 1 with only shear keys in the middle of the plate for various accelerometer locations.

The trend lines seem very consistent and alike when comparing the three specimens with the accelerometer in the middle of the composite plate. Figure 42 illustrates the frequency response for the shear key-only specimens with the accelerometer in the middle of each specimen. The only discrepancy between the values for the middle shear key-only specimens was the second specimen which showed a slight distortion from the other two trend lines. This occurrence could have been caused by not securing the specimen between the two aluminum block fixtures correctly. The screws used to tighten each aluminum block to the vibration table were screwed by a torque wrench that was consistently tightened to a value of $16 \mathrm{ft}$.-lbs. When compressing the specimen between the two aluminum blocks, the screws used to tighten the two metallic blocks together were hand-tightened. Not applying a consistent torque to these screws could have caused the second specimen to become loose in the fixture, which would have caused the slight distortion in the data when vibrating. 


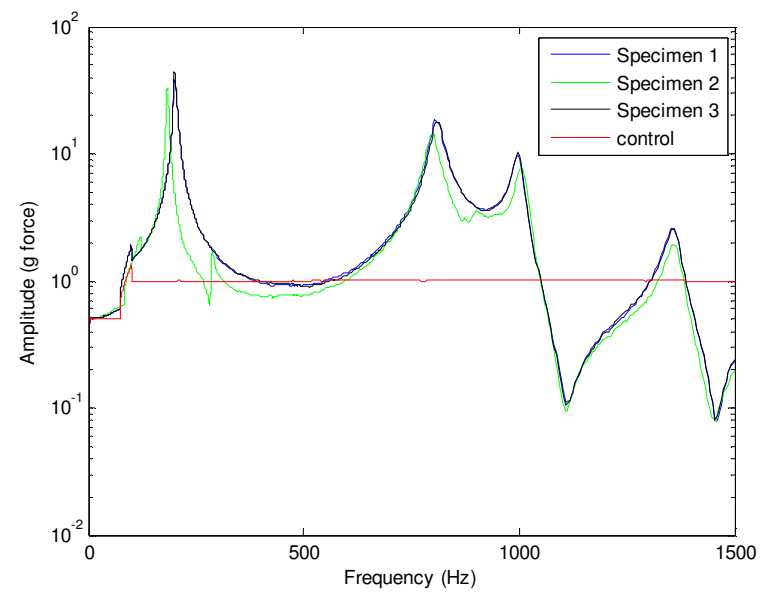

Figure 42: Frequency response for all three middle shear keys only plate specimens for when the accelerometer is placed in the middle of each specimen.

By looking more closely at the data, the slight shift in the second specimen was shown not to be detrimental to the average of the data. The relative standard deviation for the three specimens for the amplitude in the first mode had a value of $21.5 \%$. In most cases, this relative standard deviation would seem unacceptable or a verification that the data are not alike. However, when averaging three values only, it would seem to be a valid result for these circumstances. In terms of the rest of the amplitude values and the frequency values, it seems that these trends are relatively comparable to one another.
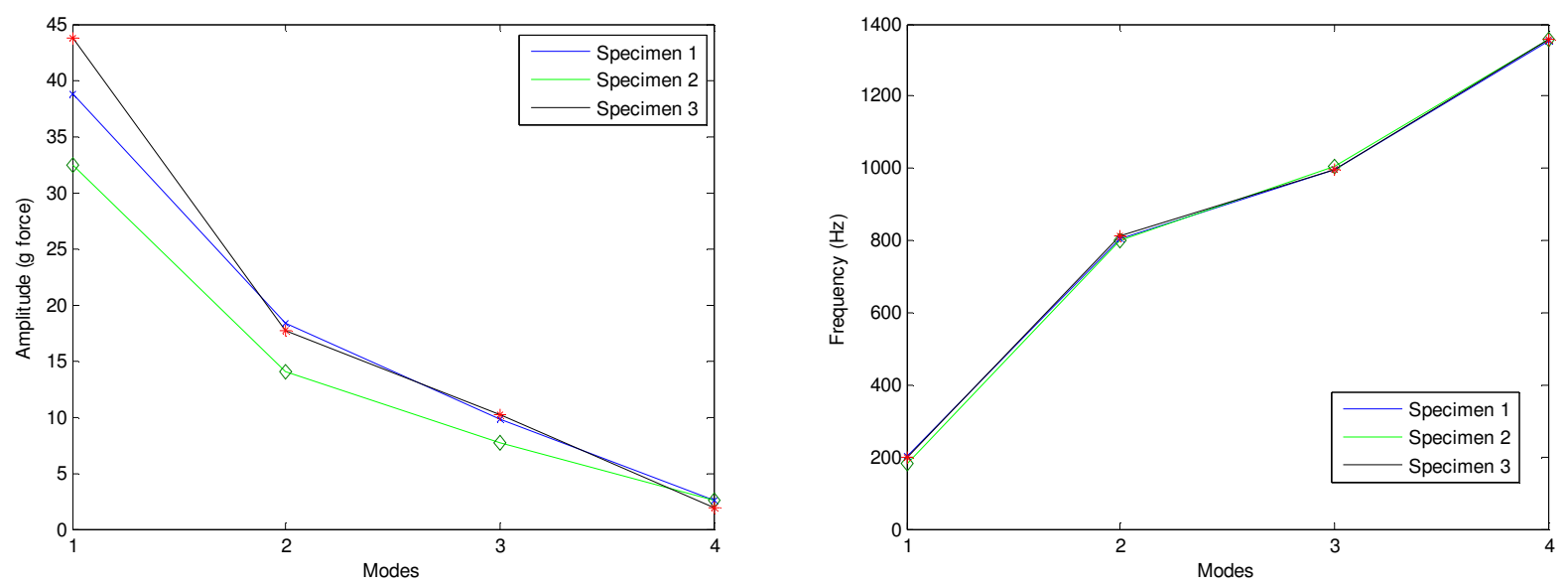

Figure 43: Amplitude and frequency response for all middle shear keys only specimens. 
Mentioned previously in the first paragraph for the shear key-only composite plate, when the accelerometer was placed off center the third mode was nonexistent. This trend is also seen in the shear only case when the shear keys were applied to the top of the specimen. Instead of having the third mode not appearing on the frequency domain, in this case it was the fourth mode. Seen on Fig. 44, only the first three modes resonated in the range of $5 \mathrm{~Hz}$ to $1500 \mathrm{~Hz}$. Once again, this would suggest that adding shear keys in the top portion of a composite plate would remove the fourth mode of a composite plate sandwich.
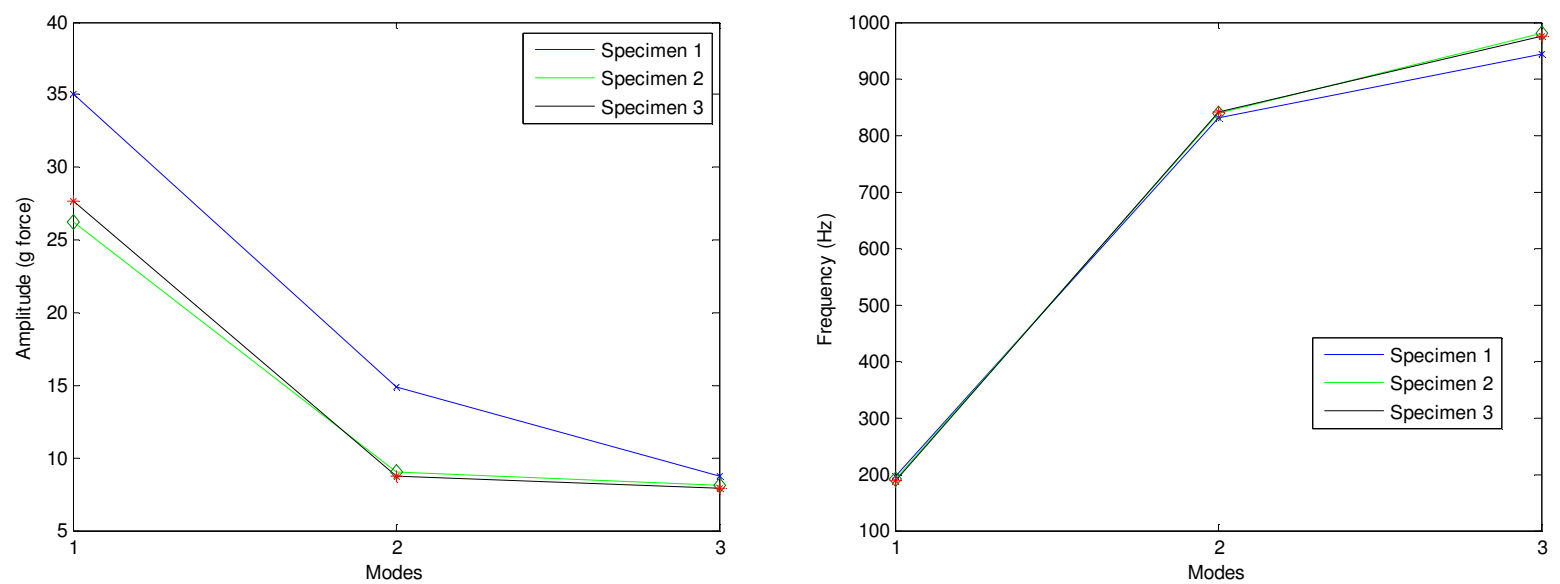

Figure 44: Amplitude and frequency response for all top shear keys only specimens.

\subsubsection{Modal Response of the Delamination Specimens with Shear Keys}

The final case that was examined for the composite plate was applying both shear keys and delamination in a specimen. Figure 45 illustrates the effects of the accelerometer placement in terms of frequency response of the delaminated composite plate with shear keys. The graph once again shows that the accelerometer placed away from the center of the plate shifted the frequency to the right. For this case study, when the accelerometer is placed one inch from the top of the specimen, the amplitude increased as it did in the previous case studies. 


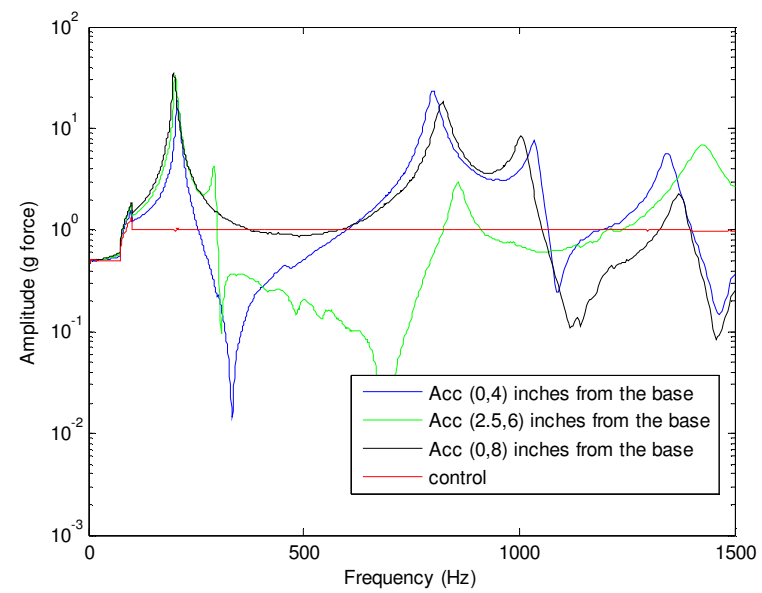

Figure 45: Frequency response for specimen 1 with the middle delamination plate with shear keys for various accelerometer locations.

When comparing each specimen with the accelerometer placed in the middle of the plate, each specimen showed a close resemblance to the other specimens. Figure 46 illustrates how the frequency response for each specimen behaves in a frequency range from 5 to $1500 \mathrm{~Hz}$. The frequency responses for all three composite plates had the most similarities in the middle case study. Both the frequency and the amplitude of all the specimens seem to lie in the same region, therefore concluding that the middle delaminated composite plate with shear keys had the least divergent values in the middle case study.

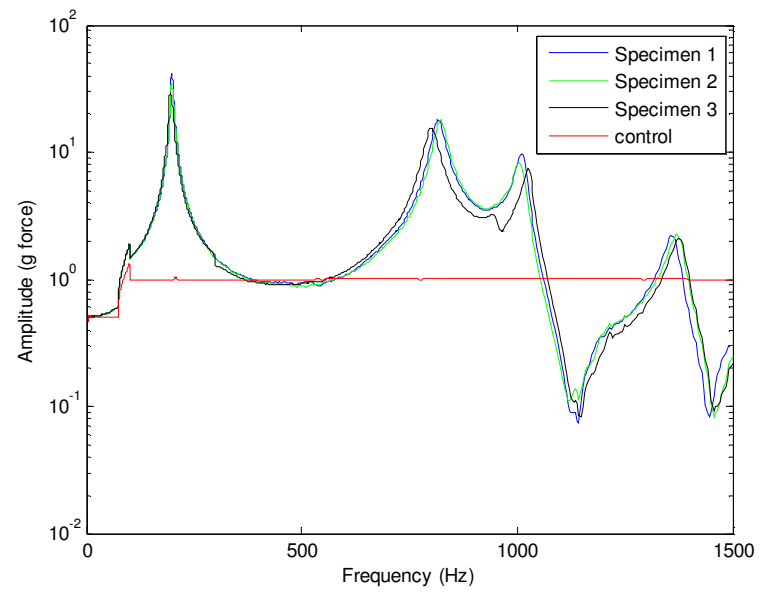

Figure 46: Frequency response for all three middle delamination with shear keys plate specimens for when the accelerometer is placed in the middle of each specimen. 
Figure 47 takes a closer look at both the amplitudes and the frequencies at each mode for the middle delaminated case with shear keys embedded into the foam. In terms of frequency, all three specimens had similar natural frequency values. However, when looking at only the amplitude of the specimens there was a minor difference in only the first natural frequency; the remaining amplitudes for the specimens were similar in value to one another. Studies of the two remaining sub-cases showed a similar trend where the highest amplitude was experienced in the first mode and continue to decrease as the composite plate experience higher frequency modes. However, the bottom and side sub-cases showed different trend characteristics.
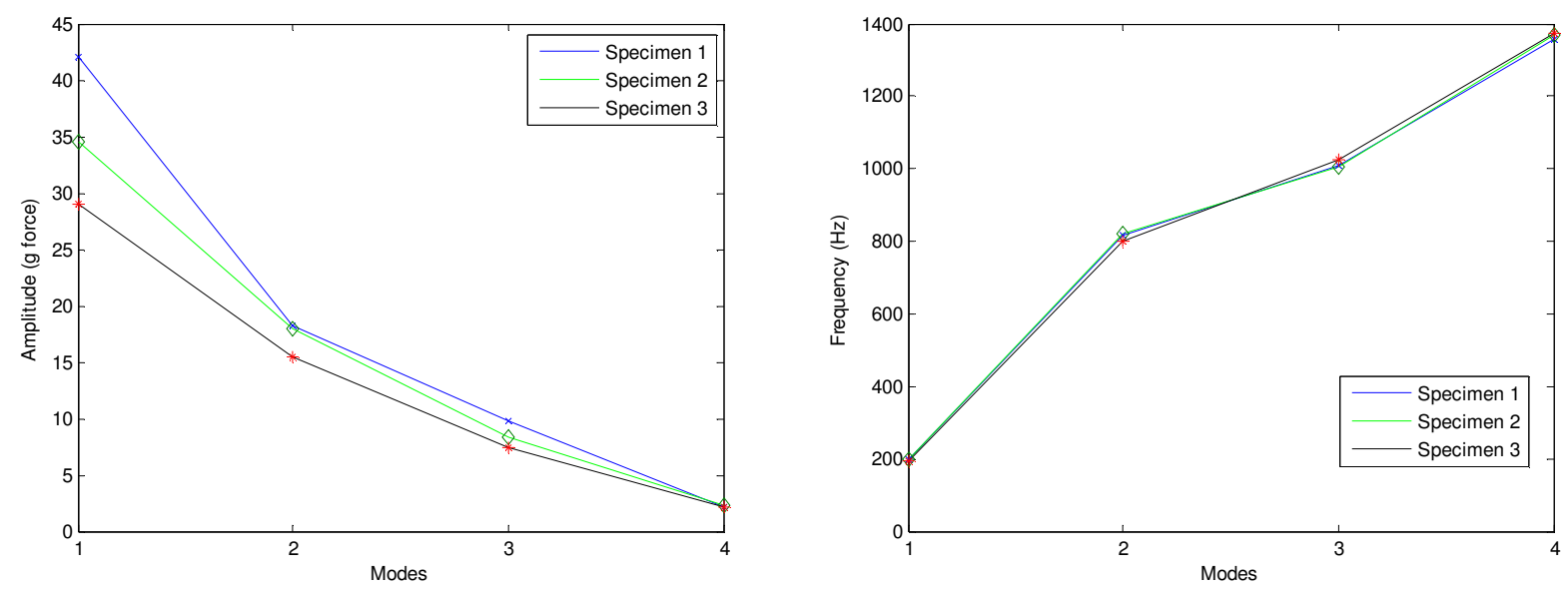

Figure 47: Amplitude and frequency response for all middle delamination specimens with shear keys.

The side and bottom sub-cases showed the same trends where the second resonance value was higher than the first resonance value in terms of amplitude. Figure 48 shows the amplitude and frequency response for the side-delaminated composite plate with shear keys in the vertical direction. Only one of the specimens had a higher amplitude in the first mode while the other two had higher amplitude values in the second mode. In the bottom case study, all the specimens had a higher amplitude value in the second mode. However, when the values of these sub-cases were compared to the values for the other sub-cases for just the first mode, there were some variances in the values. In the sub-cases where the highest mode occurred in the second resonance, the amplitude value for the first mode was around $18 \mathrm{~g}$ 's. Unlike the other sub-cases, the amplitude value for the first mode was on average around $38 \mathrm{~g}$ 's-- a difference of 20 g's. Therefore, there were some inconsistencies in the side case where specimens experienced a different amplitude trend. 

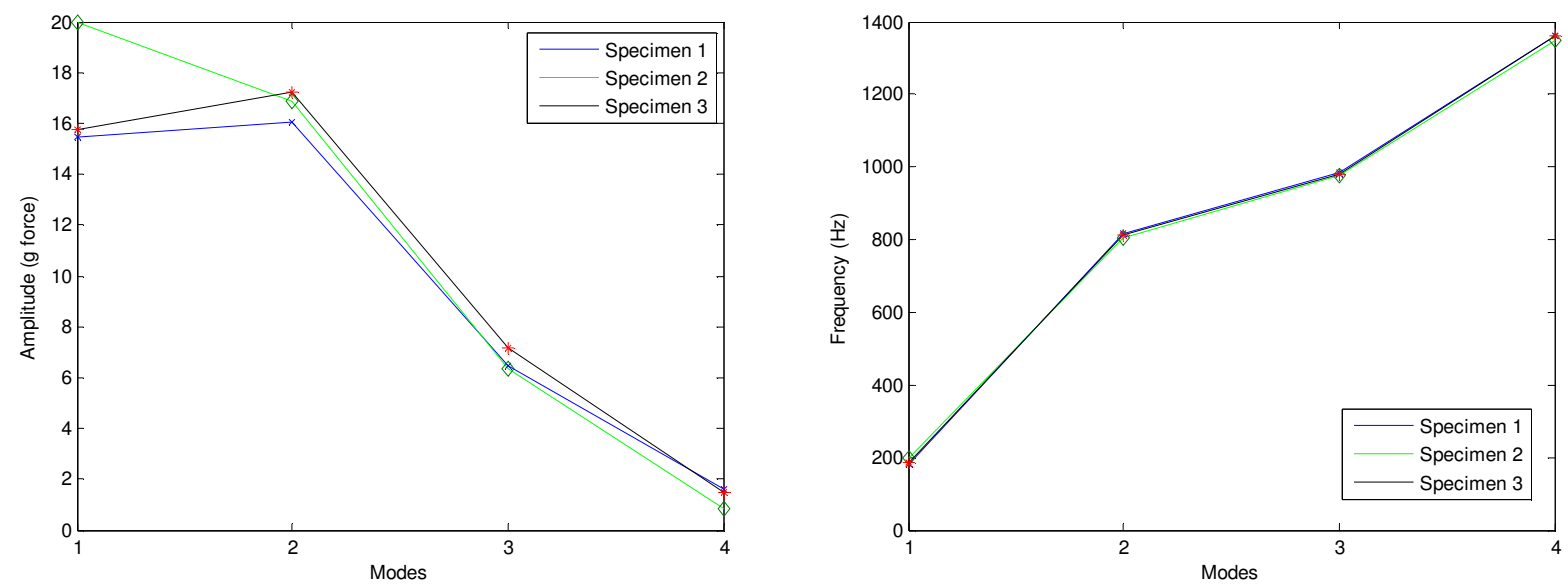

Figure 48: Amplitude and frequency response for all side delamination specimens with shear keys in the vertical direction.

\subsubsection{Comparison Between the Frequency Responses for Each Plate Configuration}

Throughout the composite plate experiment three specimens were tested for each case study. Even though having more specimens would give a better average, it would also cost more to produce more composite plates. Each plate produced would waste material that was not reusable and would also waste an abundant amount of water when curing in the autoclave. Therefore, the decision was made to acquire three plates for each case study in order to reduce the cost for this research. Having at least three composite sandwich plates per sub-case, it would greatly improve the validity of the average compared to one composite plate to base my experimental results on.

When considering the location of the accelerometer in analyzing the frequency response, it is evident that both the natural frequency and amplitude are greatly affected by the location. To further study the effect of the accelerometer location, the data were averaged for the three composite sandwich plates and then compared to the other case studies. The comparison between the composite sandwich specimens with and without shear keys and/or delamination in the middle of the plate is depicted in Table 6. These numbers once again show that the amplitude when the accelerometer is placed in the center of the specimen with a height of 8 inches from the aluminum block fixtures has the highest value when compared to the rest of the placements. The accelerometer location off center once again showed a shift in frequency and in some cases the third mode was nonexistent. 
Table 6: Comparison between the different accelerometer locations for each composite sandwich plate case study.

\begin{tabular}{|c|c|c|c|c|c|c|c|c|c|}
\hline \multirow{2}{*}{ Case Study } & \multirow{2}{*}{$\begin{array}{c}\text { Accelerometer } \\
\text { Location }\end{array}$} & \multicolumn{2}{|c|}{ Mode 1} & \multicolumn{2}{c|}{ Mode 2} & \multicolumn{2}{c|}{ Mode 3} & \multicolumn{2}{c|}{ Mode 4} \\
\cline { 3 - 10 } & Freq. & Amp. & Freq. & Amp. & Freq. & Amp. & Freq. & Amp. \\
\hline Control & Acc.4) & 211.57 & 24.43 & 822.1 & 22.31 & 1092 & 6.12 & 1379.67 & 5.66 \\
\hline Control & Acc. (2.5,6) & 211.3 & 44.21 & 882.8 & 6.613 & NaN & NaN & 1431 & 8.456 \\
\hline Control & Acc. (0,8) & 206.6 & 51.6233 & 860.3 & 18.0000 & 1034 & 9.0690 & 1440.7 & 1.6260 \\
\hline $\begin{array}{c}\text { Delamination } \\
\text { w/o Shear Keys }\end{array}$ & Acc. (0,4) & 190.2 & 39.24 & 839.53 & 17.72 & 1008.5 & 10.42 & 1395 & 1.99 \\
\hline $\begin{array}{c}\text { Delamination } \\
\text { w/o Shear Keys }\end{array}$ & Acc. (2.5,6) & 212.8 & 43.76 & 876.3 & 7.58 & 996 & 2.019 & 1387 & 8.57 \\
\hline $\begin{array}{c}\text { Delamination } \\
\text { w/o Shear Keys }\end{array}$ & Acc. (0,8) & 215.9 & 22.23 & 816.2 & 21.99 & 1059 & 6.881 & 1322 & 5.4 \\
\hline $\begin{array}{c}\text { Shear Key } \\
\text { Only }\end{array}$ & Acc. (0,4) & 194.97 & 24.88 & 806.7 & 17.86 & 999.4 & 9.81 & 1356.66 & 2.18 \\
\hline $\begin{array}{c}\text { Shear Key } \\
\text { Only }\end{array}$ & Acc. (2.5,6) & 199.3 & 37.6 & 843.8 & 1.95 & NaN & NaN & 1374 & 5.85 \\
\hline $\begin{array}{c}\text { Shear Key } \\
\text { Only }\end{array}$ & Acc. (0,8) & 206.8 & 18.1 & 792 & 19.94 & 1044 & 6.58 & 1349 & 5.25 \\
\hline $\begin{array}{c}\text { Delamination } \\
\text { w/o Shear Keys }\end{array}$ & Acc. (0,4) & 196.94 & 35.18 & 813.23 & 17.24 & 1013.33 & 8.55 & 1367.33 & 2.19 \\
\hline $\begin{array}{c}\text { Delamination } \\
\text { w/o Shear Keys }\end{array}$ & Acc. (2.5,6) & 200.8 & 31.71 & 846.5 & 3.037 & NaN & NaN & 1419 & 5.895 \\
\hline $\begin{array}{c}\text { Delamination } \\
\text { w/o Shear Keys }\end{array}$ & Acc. (0,8) & 208.5 & 17.56 & 787 & 19.95 & 1043 & 5.8 & 1345 & 5.567 \\
\hline
\end{tabular}

For each case study, four different composite orientations were needed to be compared to one another. The only trend that was identical in each case was that the control case had the highest amplitude and frequency at each mode. Figure 49 shows the comparisons between the delaminated only, shear keyonly, control, and delamination with shear key for the middle case study. The average shear key-only subcase has the lowest amplitude for the first mode and the lowest frequency values for all the modes, except for the first. By combining shear keys and delamination together, the trend is slightly higher than the shear key-only sub-case for every amplitude and frequency at each mode. The average delaminated only sub-case has the second highest amplitude for the first and second modes while having the lowest natural frequency for the first mode. These conclusions suggest that applying shear keys to a composite sandwich plate would decrease the vibration amplitude of the structure, but also decrease the natural frequency value when compared to the control specimen. 

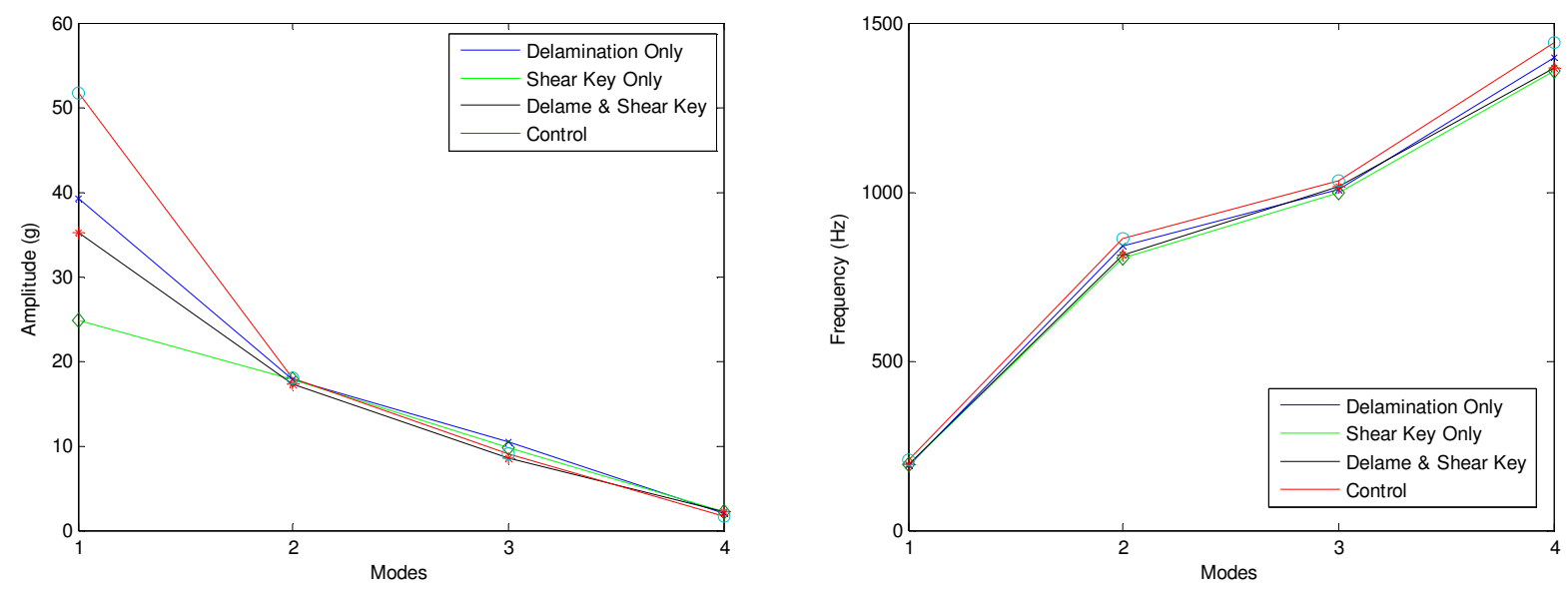

Figure 49: Average amplitude and frequency response for all cases that involve a middle delamination and/or shear keys.

Comparing the bottom case to the rest of the cases, there was a close resemblance to the top and side trends. Figure 50 shows the comparisons between the delaminated only, shear key-only, control, and delamination with shear key for the bottom case study. The average shear key-only sub-case has the second lowest amplitude for the first mode and the second highest frequency values for all the modes. As stated in the previous section, the delamination with shear keys sub-case was the only average specimen to have the highest amplitude in the second mode. This would explain why the first amplitude value was lower than the second amplitude value. The average delaminated-only sub-case had the second highest amplitude for the first mode while having the lowest natural frequency for all four modes of the experiment. These data suggest that applying shear keys to a composite sandwich plate would significantly decrease the amplitude, due to vibration, of the structure while slightly decreasing the natural frequency value when compared to the control specimen. 

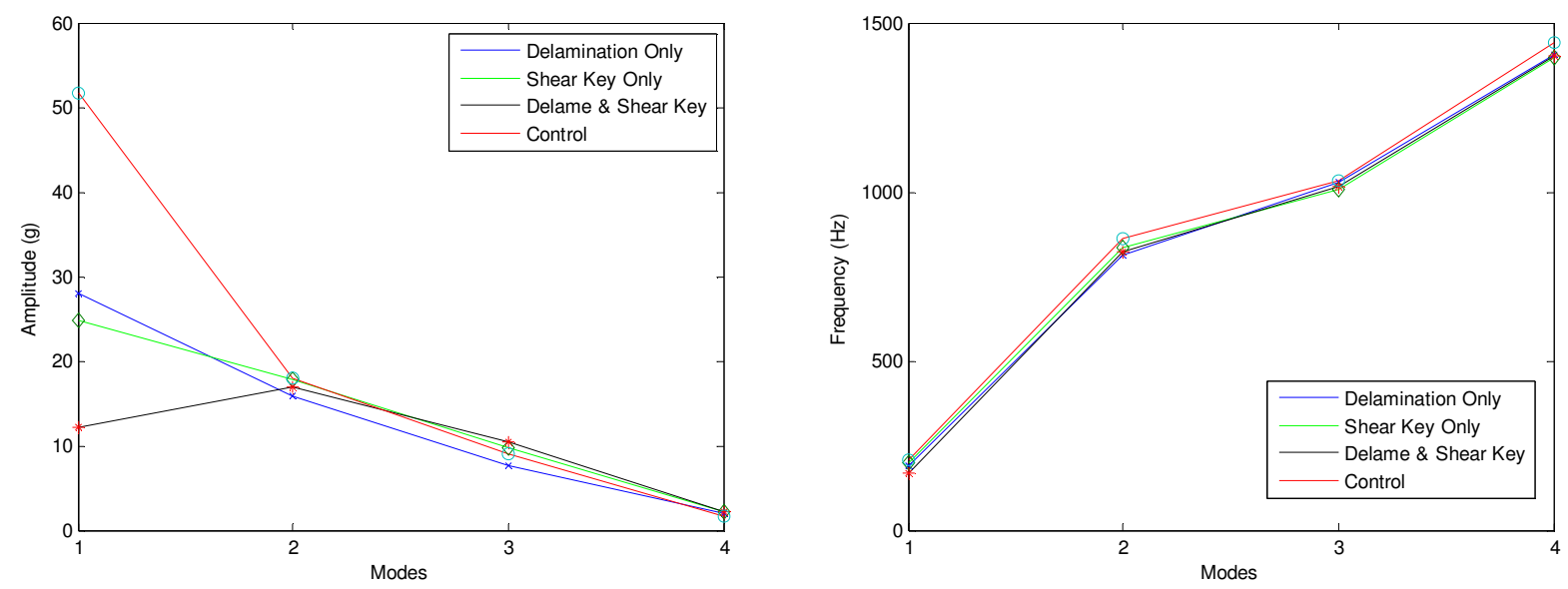

Figure 50: Average amplitude and frequency response for all cases that involve a bottom delamination and/or shear keys.

The top case closely resembled bottom and side trends as stated in the previous paragraph. Figure 51 shows the average amplitude and frequency response for all cases that involved a top delamination and/or shear keys. The average shear key-only sub-case had the second lowest amplitude and the second highest frequency values for all the modes. The average delamination with shear keys embedded into the foam sub-case had the lowest values for both amplitude and frequency for all the modes in the experiment. The average delaminated-only sub-case had the second highest amplitude for all the modes. This sub-case also had a fluctuating frequency, having one of the highest frequencies at one mode and the lowest in another mode. These trends suggest that applying shear keys to a composite sandwich plate would significantly decrease the amplitude of the structure while slightly decreasing the natural frequency value when compared to the control specimen. 

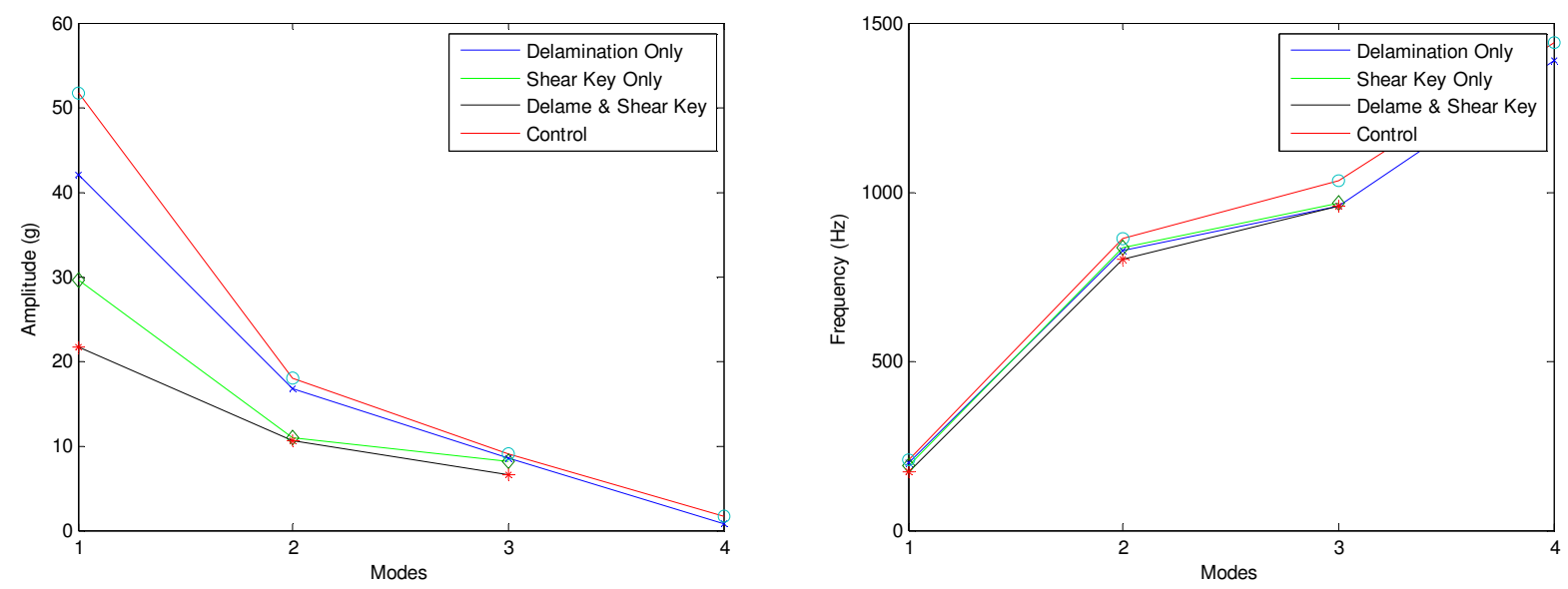

Figure 51: Average amplitude and frequency response for all cases that involve a top delamination and/or shear keys.

The only cases that had identical trend lines were that of the side case and the top case. The values between each sub-case were different from one another, but the order in which each sub-case would decrease in value was the same. Figure 52 shows the relationship between all the averaged subcases for the side vertical case. The amplitude was identical to the top case where the control specimen experienced the highest amplitude value for all the modes, which was then followed by the delaminated, shear key-only, and delamination with shear key sub-case, in that respective order. However, there was a slight change in the frequency order with the shear key-only sub-case and delaminated only sub-case altering their positions in respect to the other cases. Nonetheless, these trends show familiar results in which specimens with shear keys would decrease the amplitude significantly and slightly reduce in frequency in each mode when compared to the control specimen and the delaminated specimens.
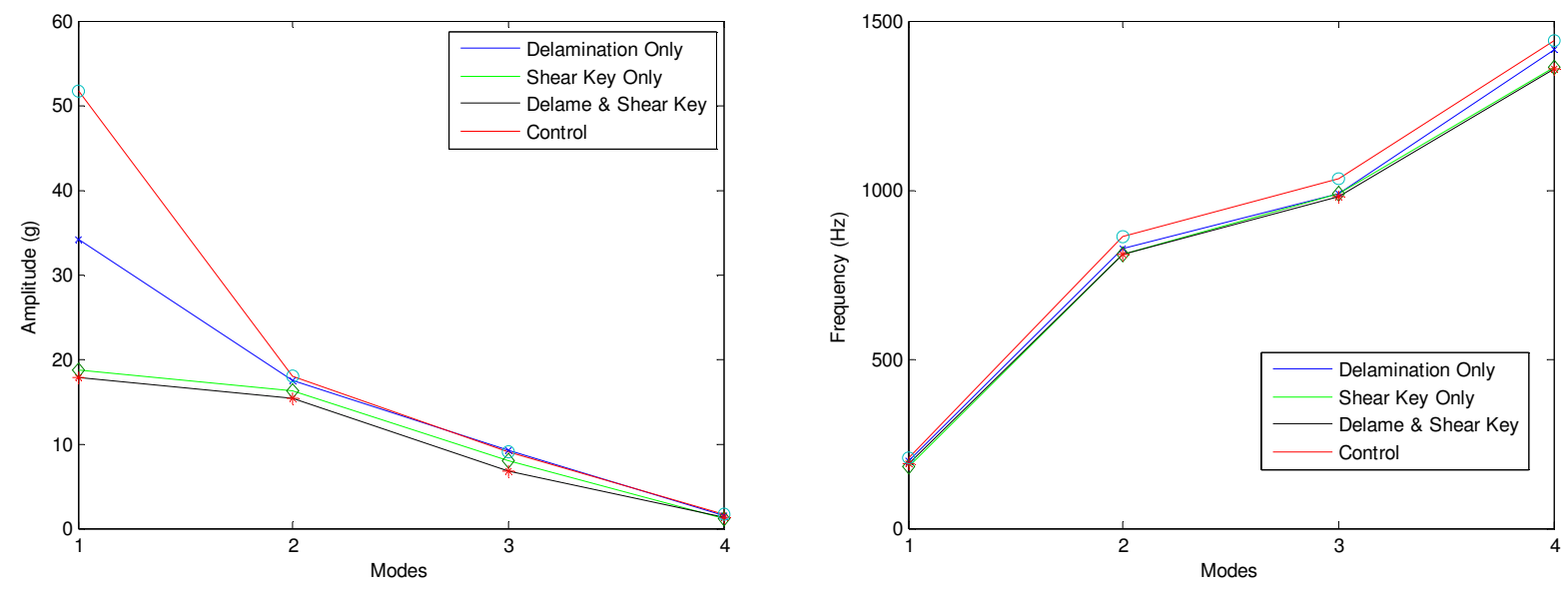
Figure 52: Average amplitude and frequency response for all cases that involve a side delamination and/or shear keys in the vertical direction of the plate.

The final case study had the greatest change in terms of amplitude when compared to the previous composite sandwich plate cases. The frequency trends are similar to the other cases where the lowest frequency values were obtained from the delaminated composite. The values for composite sandwiches with shear keys were between the control and delaminated-only sub-cases. However, when analyzing the amplitude results from all the sub-cases, there were dissimilarities when compared to the rest of the case studies. Figure 53 shows the relationship between all the averaged sub-cases for the middle vertical case in terms of frequency and amplitude. Unlike the previous examples, the average delaminated-only subcase had the lowest amplitude when compared to the rest of the sub-cases. The second highest amplitude was from the delaminated composite with shear key. From these trends, the conclusion is that composite sandwich specimens that are delaminated will have a lower amplitude, but at a cost of having a lower frequency value at each mode. Composite sandwich plates with shear key in the vertical position will acquire a higher frequency and amplitude than a delaminated specimen, but will have lower resonance frequency values when compared to a control specimen.
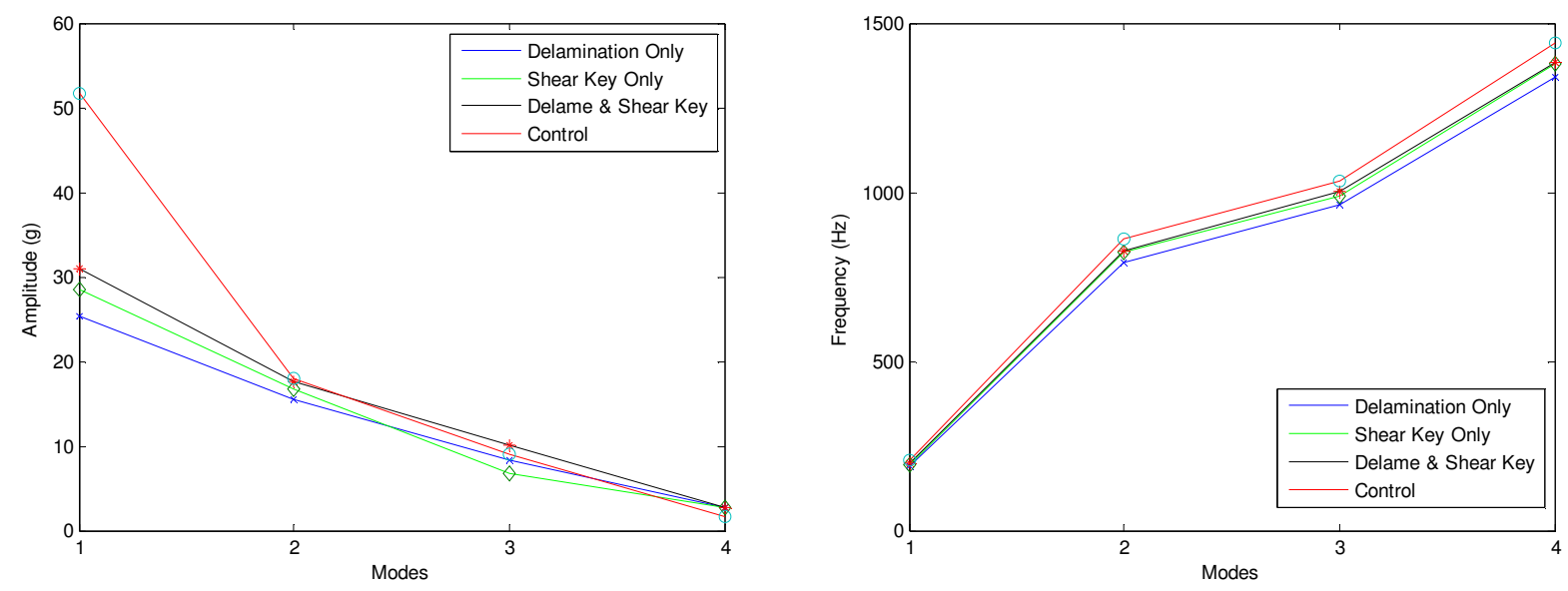

Figure 53: Average amplitude and frequency response for all cases that involve a middle delamination and/or shear keys in the vertical direction of the plate.

\subsection{Dynamic Response of the Composite Sandwich Beams}

To further investigate behaviors of shear keys under dynamic loading the composite plate samples were cut into beam specimens. Unlike the plate case where a limited number of plates were 
vibrated for each case, the number of beams that could be produced increased from 3 to 7 specimens. This was possible due to the fact that the specimens were cut from the plates that were previously vibrated. The amount of seven was chosen based on the various ASTM standards that were used when acquiring material properties. These ASTM standards recommended at least five to seven samples be taken for each test. Therefore, each case had at least 28 specimens to acquire data from.

To determine the full effect of the damage arrestment devices, several configurations were needed which included: control specimens, delaminated specimens, shear keys only specimens, and a combination of shear keys and delamination. These beams were placed onto the vibration table to undergo nodal analysis. Once again, one important aspect of screwing these beams onto the table was the alteration of the geometry. The one-inch aluminum blocks would only permit $90 \%$ of the actual composite plate to be free to vibrate. Even though each beam was $10 \times 1$ inches in area, only the $9 \times 1$ inch section of the beam would be allowed to experience the vibration effects.

\subsubsection{Modal Response of the Control Specimens}

In the previous section where the composite sandwich plates were analyzed, the accelerometer was placed in the middle of the specimen in order to limit the effects of the accelerometer's mass on the frequency response of the composite. However, knowing that the accelerometer is more likely to detect bending resonance than torsional resonance, proposed that the accelerometer location be changed. Having the accelerometer in the middle of the specimen would greatly affect the amplitude of the second mode. The reason behind this is that the middle of the beam would not experience as much movement due to the middle of the structure being essentially fixed while experiencing the second mode. In the second mode, the ends and the middle will remain in place while the other portions oscillate in a sinusoidal fashion. This mode shape has the characteristics of a typical cantilever beam and could possibly affect the results acquired from the accelerometer. Therefore, the accelerometer was placed in the middle of the specimen and at a height of 9 inches from the bottom of the composite plate.

The experimental data obtained from the Winll software were then transferred to the software program Matlab to be assimilated for analysis. Figure 54 shows the frequency response of all the specimens from the control beam case study. Using the same frequency range from the previous experiment, only two resonances occurred on the composite sandwich beam. At first glance, most of the specimens seemed to follow a similar trend, except for the second specimen. There are several explanations for this phenomenon which includes the specimen not being tightened enough between the two aluminum blocks or the specimen being over-tightened, and possibly fractured or delaminated in an 
unwanted location. Nonetheless, this sample was taken out of the averaging process of the control composite sandwich beam.

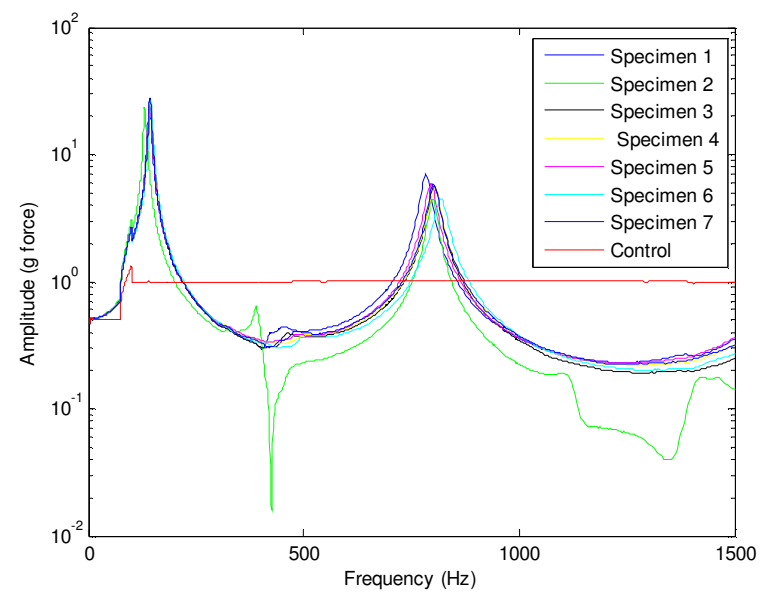

Figure 54: Frequency responses for all composite sandwich control specimens.

\subsubsection{Modal Response of the Delaminated Specimens}

The composite sandwich plate samples had a delamination placed in five different orientations to determine whether the orientation of the delamination affected the frequency response of the composite specimen. However, for the beam case, only three case studies included the top, bottom, and middle. The vertical cases could not be placed on the composite sandwich beam due to the fact that the delamination width had a finite value of $1 \mathrm{inch}$. Therefore, if a delamination was applied to the beam geometry then the entire facesheet and core sandwich would be completely delaminated from one another. For this reason only, the middle, bottom, and top sub-cases were analyzed for the composite sandwich beams.

Throughout the entire experiment for both the composite sandwich plates and beams the accelerometer was placed on the side where the delamination and/or shear keys were placed. To determine whether the accelerometer's location on either the front side or back side of the composite specimen affected the frequency response, the accelerometer was placed opposite from the delamination for the middle sub-case. Figure 55 shows the frequency response for the middle delaminated composite sandwich beam with seven specimens and one case where the accelerometer was placed on the opposite side of the delamination. From the graph, it was determined that having the accelerometer on the back side or front side of the composite structure did not affect the results of the accelerometer response. 
However, if the placement was shifted up or down from the top portion of the specimen then the accelerometer would generate a different response trend.

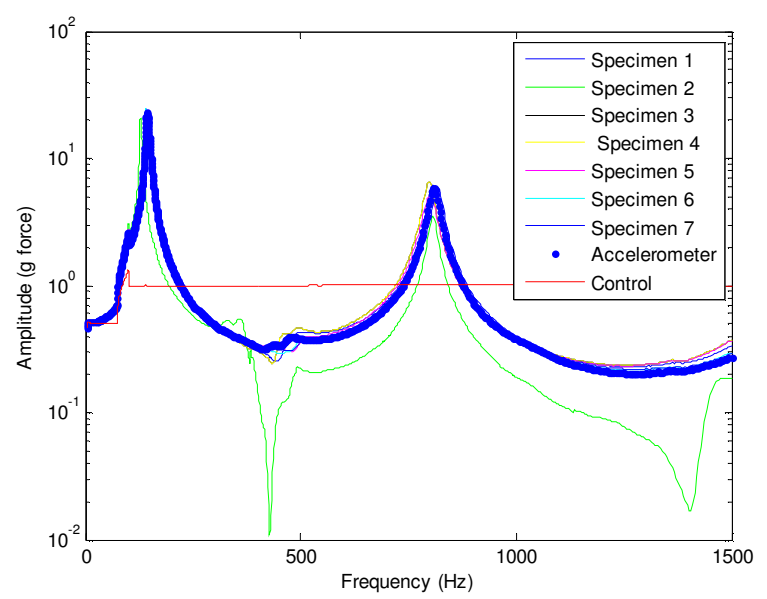

Figure 55: Frequency responses for all middle delaminated composite specimens.

\subsubsection{Modal Response of the Shear Key-only Specimens}

The shear keys that were embedded into the composite sandwich beam structure were placed horizontally in the beam. In another experiment, that will be explained in later sections, the shear keys were placed in the middle of the beam with a length of 10 inches. However, for this experiment and for the remaining composite sandwich beam cases, the shear keys had a length of 1 inch and were placed horizontal and parallel to the front end of the vibration table. It was also important to confirm that the outermost fibers were perpendicular to the surface of the vibration table. Once confirmed that the beams had the correct orientation, the experiment was begun. Figure 56 illustrates the vibration response for the middle shear key sub-case for all the specimens that were vibrated. Unlike the previous examples, all the specimens had related trends and similar values. 


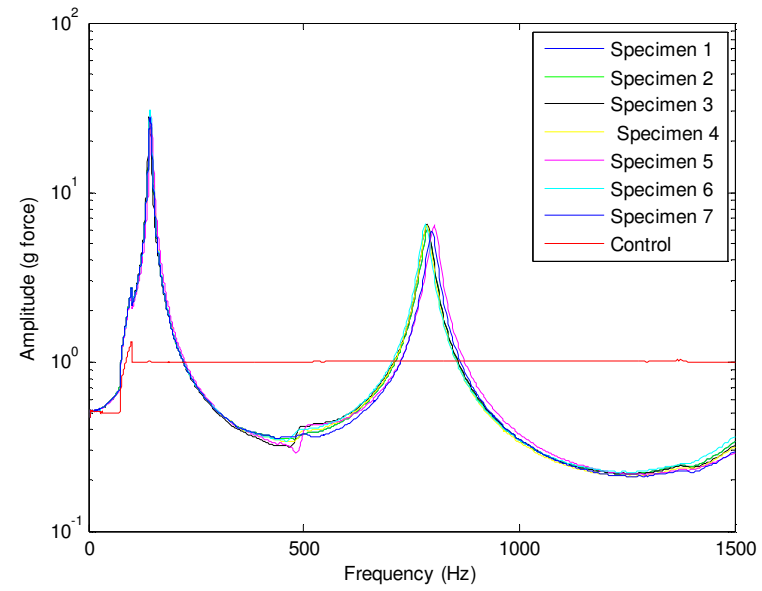

Figure 56: Frequency responses for all middle shear keys only composite specimens.

\subsubsection{Modal Response for the Shear Key with Delamination Specimens}

The final experiment for the composite sandwich beam involved having both the delamination and the shear keys between the foam core and carbon fiber facesheet. Figure 57 illustrates the frequency response of the middle sub-case with both shear keys and delamination in the lay-up. For this case study, almost all the amplitude and natural frequency values for the first mode were nearly identical to one another. The relative standard deviation for the middle sub-case at the first mode was $1.24 \%$ and $1.79 \%$ for the natural frequency and amplitude values, respectively. Therefore, the specimens for the delaminated composite beam with shear keys had the most consistent values throughout the research.

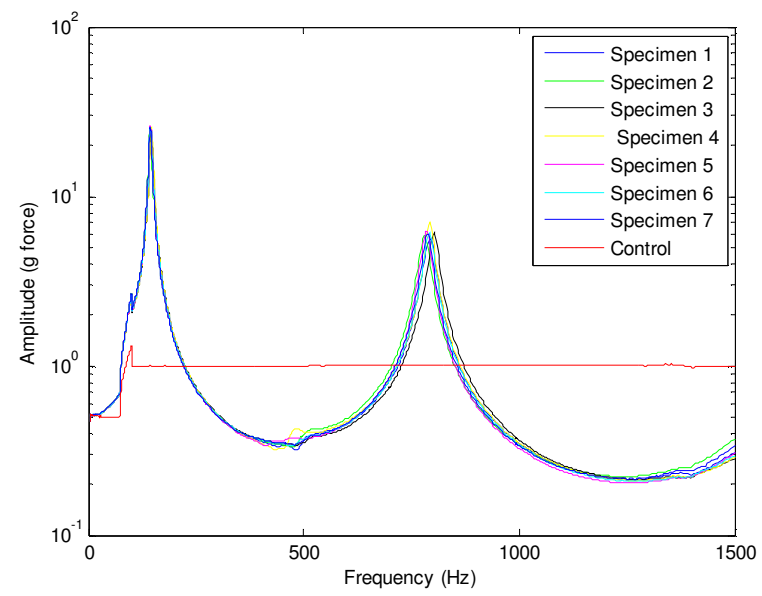

Figure 57: Frequency responses for all middle delaminated composite specimens with shear keys. 


\subsubsection{Comparison Between the Frequency Response for Each Beam Configuration}

For each case study, four different composite orientations were needed for comparison to one another. In all the case studies, the delaminated sub-case had the lowest amplitude and frequency for the first mode. Figure 58 shows the comparisons between the delaminated only, shear key-only, control, and delamination with shear key for the middle case study. The average shear key-only sub-case had the highest amplitude for the first mode and had the second highest frequency values for the first mode. In terms of amplitude, the average specimen with delamination and shear keys inside the composite behaved similar to the control case. However, in terms of natural frequency it was slightly higher than the rest of the sub-cases for the first mode. These conclusions suggest that applying shear keys to a composite sandwich plate would increase the amplitude of vibration of the structure, but would stay relatively at the same natural frequency value when compared to the average control specimen and the average delaminated specimen value without shear keys.
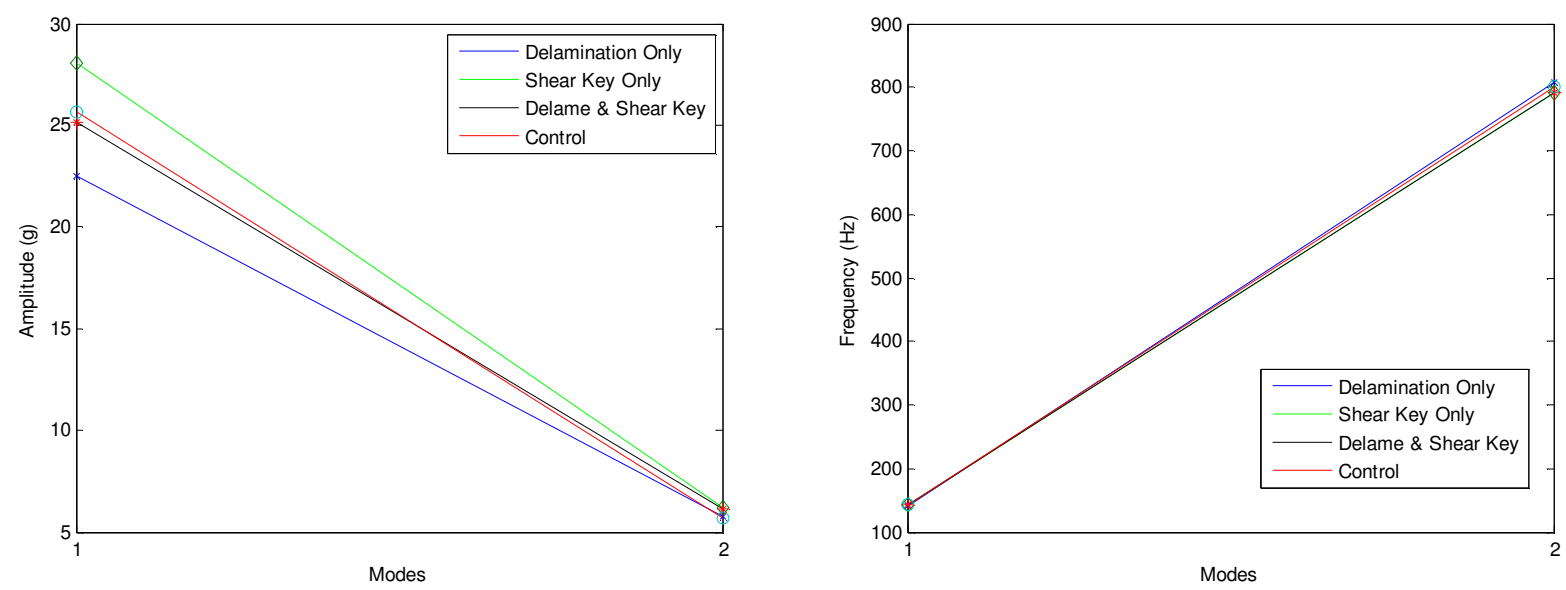

Figure 58: Comparison between the Average amplitude and frequency response for all cases that involve a middle delamination and/or shear keys.

Comparing the bottom case to the rest of the cases, there is a closer resemblance among the top case study than the middle case study. Figure 59 shows the comparisons between the delaminated only, 
shear key-only, control, and delamination with shear key for the bottom case study. The average shear key-only sub-case had the highest natural frequency value for the second mode. In terms of amplitude the average shear key-only sub-case behaved similarly to the control case for all the modes. The delaminated composite beam with shear keys embedded into the foam had the highest amplitude value for each mode. In terms of frequency, the shear key with delamination average specimen had the second highest frequency at every mode. The data suggest that applying shear keys to a composite sandwich beam would increase the amplitude and the natural frequency value when compared to the control specimen and the delaminated composite sandwich beam without shear key in the structure. However, the frequency would only be increased in the second mode and not in the first mode.
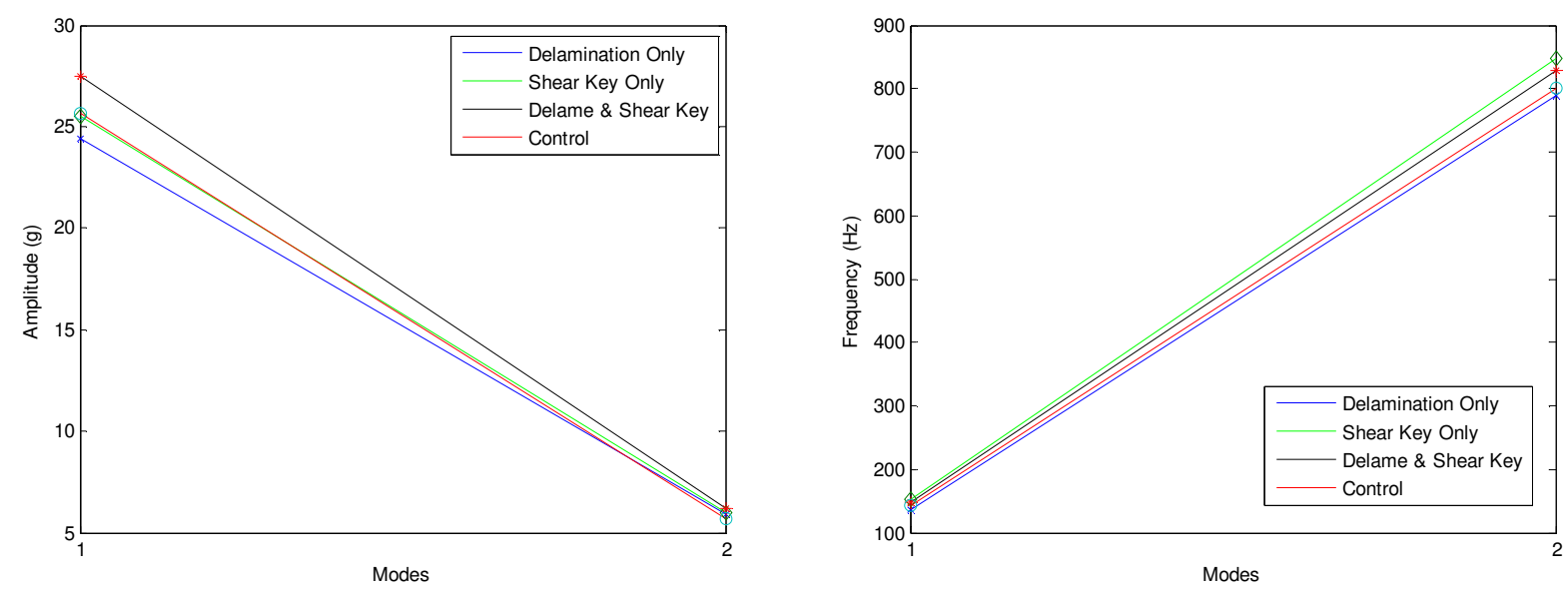

Figure 59: Comparison between the Average amplitude and frequency response for all cases that involve a bottom delamination and/or shear keys.

The top case closely resembled the bottom case as stated in the previous paragraph. Figure 60 shows the average amplitude and frequency response for all cases that involved a top delamination and/or shear keys. This figure is entirely similar to the bottom case study with the exception of the delaminatedonly sub-case in terms of amplitude. The average delaminated-only sub-case had the lowest amplitude value for the first mode and would typically reside towards the lower values in the second mode. However, for this case, the delaminated-only sub-case had the highest amplitude value for the second mode. Without this occurrence, the bottom and top cases would have identical frequency responses for each sub-case. The data suggest that applying shear keys to a composite sandwich beam would increase the amplitude and the natural frequency value when compared to the control specimen and the 
delaminated composite sandwich beam without shear key in the structure which is similar to the bottom cases where the frequency would only be increased in the second mode and not in the first mode.
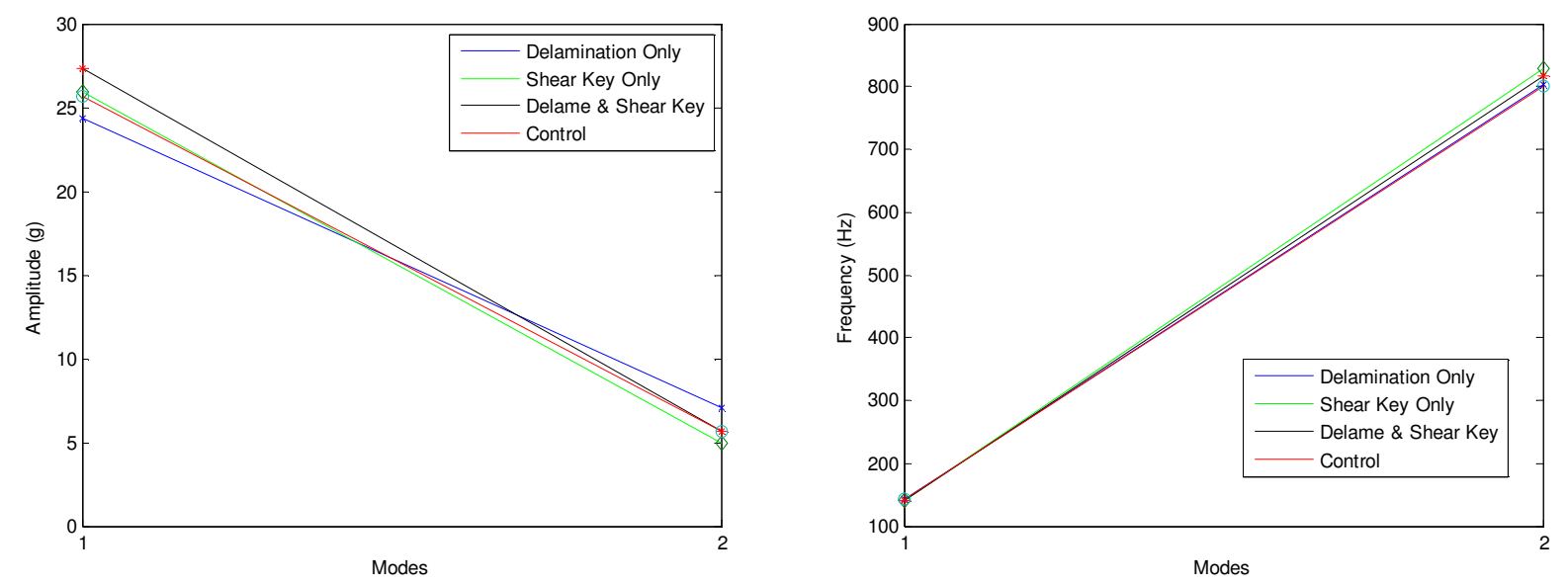

Figure 60: Comparison between the Average amplitude and frequency response for all cases that involve a top delamination and/or shear keys.

In terms of composite sandwich beams, there are similarities and differences between the three case studies. The bottom case and top case had identical trend lines when comparing the amplitude and frequency values at each mode. The middle case study led to a completely different conclusion when analyzing the data from the vibration table. The conclusion made from the middle case was that when applying shear keys to a composite sandwich beam, it would increase the amplitude only, and the natural frequency value would remain relatively the same as the control specimen. This would suggest that applying shear keys to a delaminated composite sandwich would decrease the structure's integrity instead of improving the structure's strength. However, the conclusion based on the bottom and top case would suggest that applying shear keys to a composite sandwich beam would slight help the frequency values for each mode. Nonetheless, both conclusions confirm that applying shear keys to a composite sandwich beam would increase the amplitude and could possibly cause failure due to higher amplitude.

\subsection{Altering the Delamination Region for Composite Sandwich Beams}

Throughout the entire experiment, the delamination region had remained the same with a constant width of one inch. However, in reality, when composites are delaminated, the delamination region will 
not have a consistent delamination of one inch. Therefore, another experiment was conducted to determine the effects of increasing the percentage of the delaminated region in terms of area. For beams described previously, the total area delaminated throughout the entire structure was $10 \%$. This experiment will increase the total delaminated area was increased from $10 \%$ to $30 \%$ to determine how the structure would react in terms of vibration. Similar cases will be studied with a control, delaminated only, shear key-only, and delamination with shear key cases. Along with increasing the delamination region, the orientation of the shear keys was also changed to an orientation that ran parallel to the length of the beam. The location of the delamination will be in the center of the structure and was gradually be increased by half an inch until the total delaminated region was 3 inches in length and 1 inch in width. An illustration of these three different delamination regions can be seen in Fig. 61 where the gray region represents the delamination region and the black portion represents the composite facesheet.
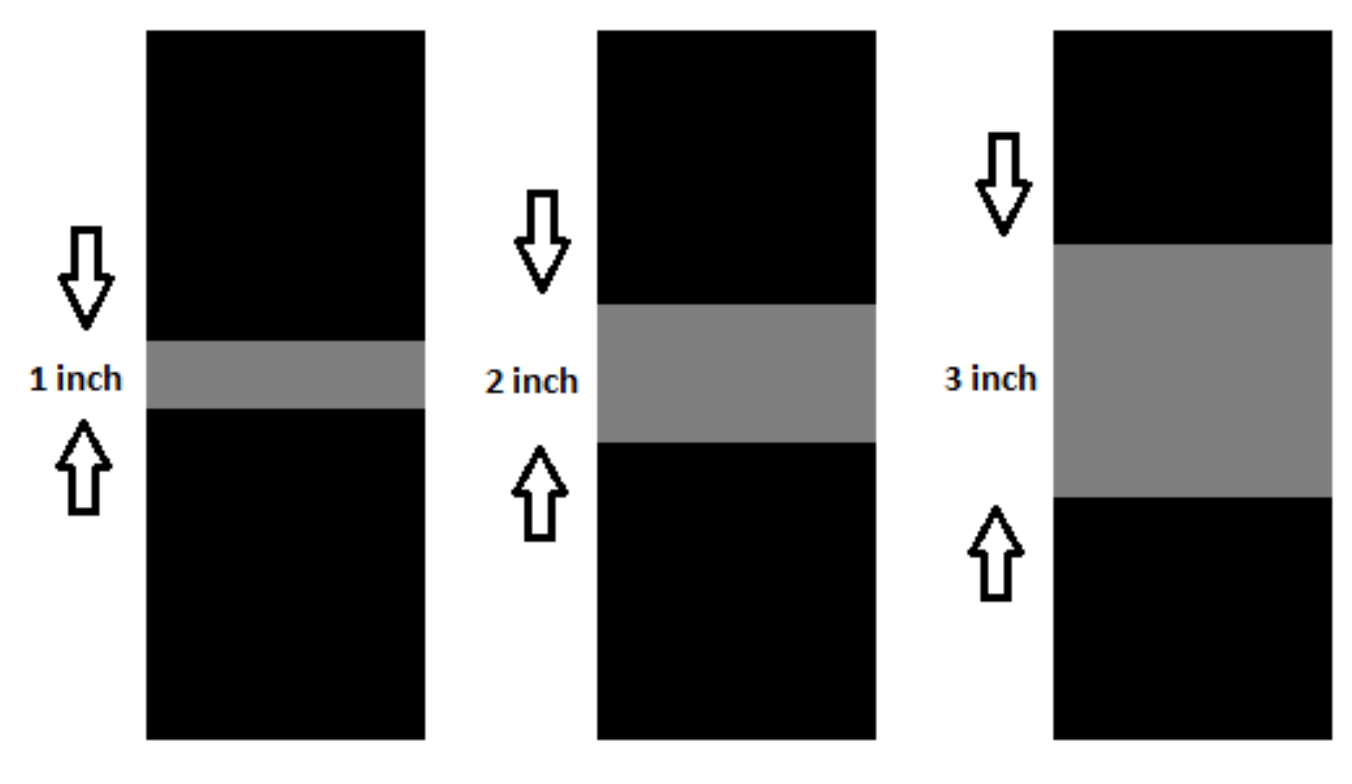

Figure 61: The various delamination regions for the vertical shear key composite sandwich beam case study.

For this iteration only, three specimens were produced for each case study. The only prior data that were used for this experiment were from the control case for the composite sandwich beam. Figure 62 illustrates the vibration response that was provided by the WinII software. The graphical representation of the frequency response shows all the cases that were examined for this experiment for the first sample of each sub-case only. The first specimen for each case was acquired and plotted to show the comparisons between the data. The results show that there were no consistencies between the data and the trends of the frequency responses for each sample. However, there was a slight relationship among the 
specimens with shear keys embedded into the foam. Even though the values were not similar, the resonance for each specimen seemed to peak at a related frequency. To fully understand how the frequency and amplitude are changing with the delamination region increasing, the specimens for each case need to be averaged and compared in a similar fashion as before.

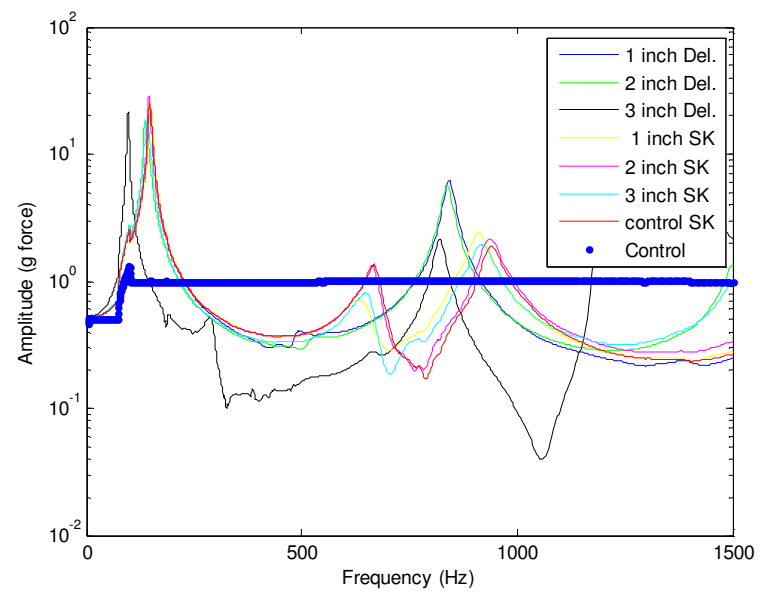

Figure 62: Frequency response for all first specimens for each case study.

By averaging the specimens at each mode, the frequency and amplitude were acquired and plotted in Matlab to more easily understand the effects of increasing the delamination region. Figure 63 is the graphical representation of the average amplitude and frequency response for all composite sandwich beam cases with their respective delamination lengths. In terms of amplitude, the specimens with shear keys continued to have higher amplitudes for the first mode, as seen in the previous beam cases. However, these specimens with shear keys running parallel with the length of the beam had lower amplitudes for the second mode when compared to the specimens without shear keys embedded into the foam. Typically in experimental analysis there are several cases that are puzzling. This is the case with the 3-inch delaminated composite beam with a shear key inside the structure. The characteristics for the amplitude are similar to those that are delaminated cases without shear keys. This would suggest that the characteristics of a delamination that is decreasing the amplitude in the first mode were incorporated in the 3-inch delaminated composite sandwich beam with a shear key.

When considering the frequency of the specimens there was a key relationship between the delaminated case and the shear key case. The graphical representation of the frequency response for each composite beam showed a separation between various trend lines. The reason is because the specimens with a shear key performed better than their respective counterpart at every mode. The 1-inch delaminated beam with shear keys had a higher frequency than the one inch delamination beam without shear key and the rest of the pairs followed a similar trend. When comparing the 3-inch delaminated specimens, a 
significant difference was seen between the specimens with and without a shear key embedded into the structure. The frequency value for the first and second resonance for the 3-inch delaminated specimen was 96.84 and 820.1, Hz respectively. The frequency value for the first and second resonance for the 3inch delaminated specimen with shear keys was $139 \mathrm{~Hz}$ and $916.3 \mathrm{~Hz}$, respectively; a significant decrease of $42.16 \mathrm{~Hz}$ for the first resonance frequency. This significant increase could possibly prevent a failure if the structure needs to have a relatively high natural frequency for the first mode.
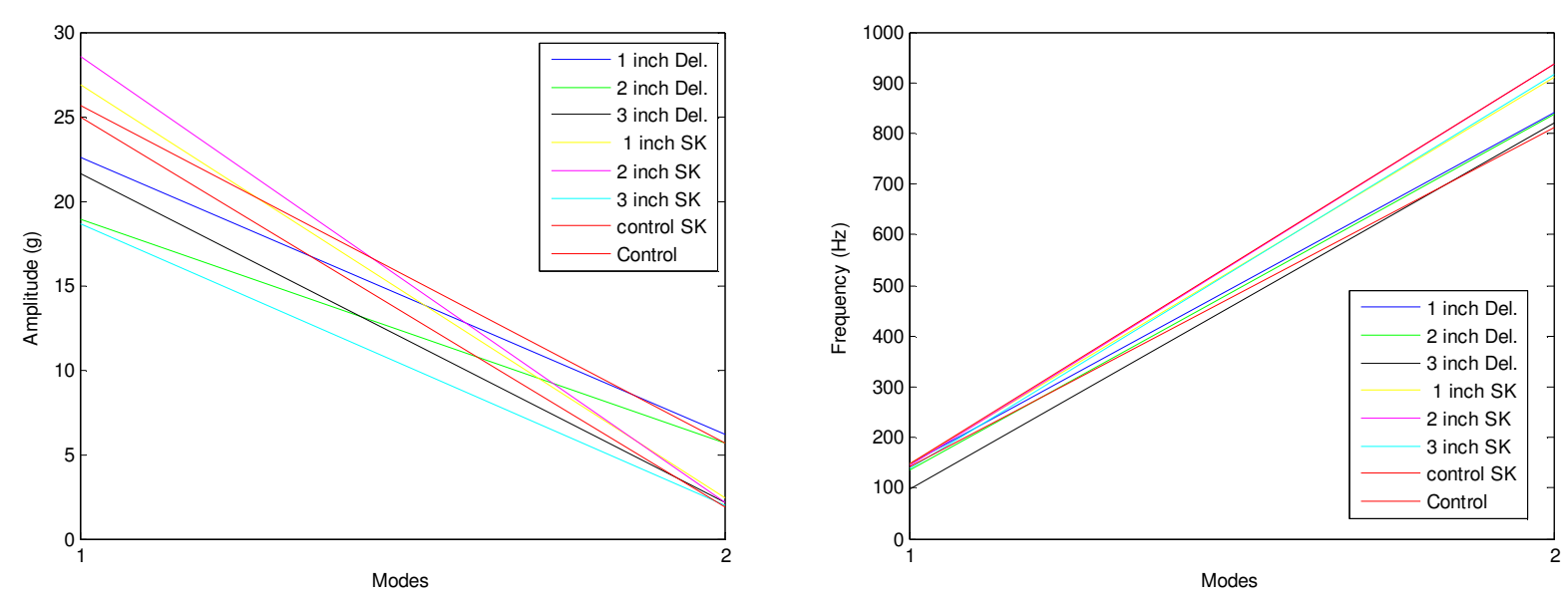

Figure 63: Average amplitude and frequency response for all composite sandwich beam cases with their respective delamination length.

\subsection{Time Response for Various Composite Specimens}

The vibration motion for various structure models decays with time. For each specimen the decay varied due to different internal and external dampening characteristics. For this experiment, the material properties did not change, but the dampening ratio and the stiffness did for each specimen. When a delamination region is introduced into a structure, the strength and frequency response are affected. Along with changing these parameters, the natural dampening ratio also varied for these specimens. Using Eq. 3, the dampening ratios for all the tested carbon fiber sandwich specimens was determined.

$$
\zeta=\frac{1}{2 \pi j} * \ln \left(\frac{\ddot{u}_{i}}{\ddot{u}_{i+j}}\right)
$$

For lightly damped systems, the dampening ratio can be determined with the above equation. The dampening ratio $\zeta$ is a function of the amplitude $\ddot{u}$ of the specimen. Every specimen will have its own 
dampening ratio because the amplitudes are not the same for each case. The average amplitude from each sub-case will be used when calculating the dampening ratio. Once the dampening ratio is calculated, the characteristic equation can now be computed. Equation 4 is used to determine the two roots for each mode of the system.

$$
s_{1,2}=\omega_{n}\left(-\zeta \pm i \sqrt{1-\zeta^{2}}\right)
$$

The characteristic equation $s_{1,2}$ is a function of the natural frequency for a particular mode and the dampening ratio of the system. For the sandwich plate, a total of four modes were detected during the experimental analysis. Therefore, four characteristic roots were calculated. For the sandwich beam cases, only two roots were obtained based on the fact that only two modes were identified during the experiment. These roots were then placed into Fourier transform function in Eq.5 to determine the time domain of each test specimen.

$$
u(t)=\sum A_{x} e^{s_{x} t}
$$

Essentially the time domain for each specimen is a function of the dampening ratio, characteristic roots, time $\mathrm{t}$, and amplitude of the mode $A_{x}$. As previously mentioned the summation is based on the number of modes determined from the experimental analysis. Therefore, a total of four summations are needed for the plate case and two summations are needed for the beam case. Once the amplitude of the system is calculated, the amplitude is plotted as a function of time. Figure 64 shows the time response for the bottom plate case and for the middle case. For this experiment all the sub-cases of the plates resembled a similar trend as the bottom case. This illustration shows that the time domain for the delamination only, shear key-only, and delamination with shear key sub-cases all dampen approximately at the same time. However, the control sub-case, for all the plate cases, dampens faster than the rest of the sub-cases. This would conclude that even with the assistance of shear keys embedded into the foam, the time response of a delaminated specimen would still falter in comparison with a specimen without any delamination. In some cases, such as the middle case, applying shear keys actually decreases the dampening ratio and as a result the system takes a very long time to dampen the amplitude exerted by the vibration. 

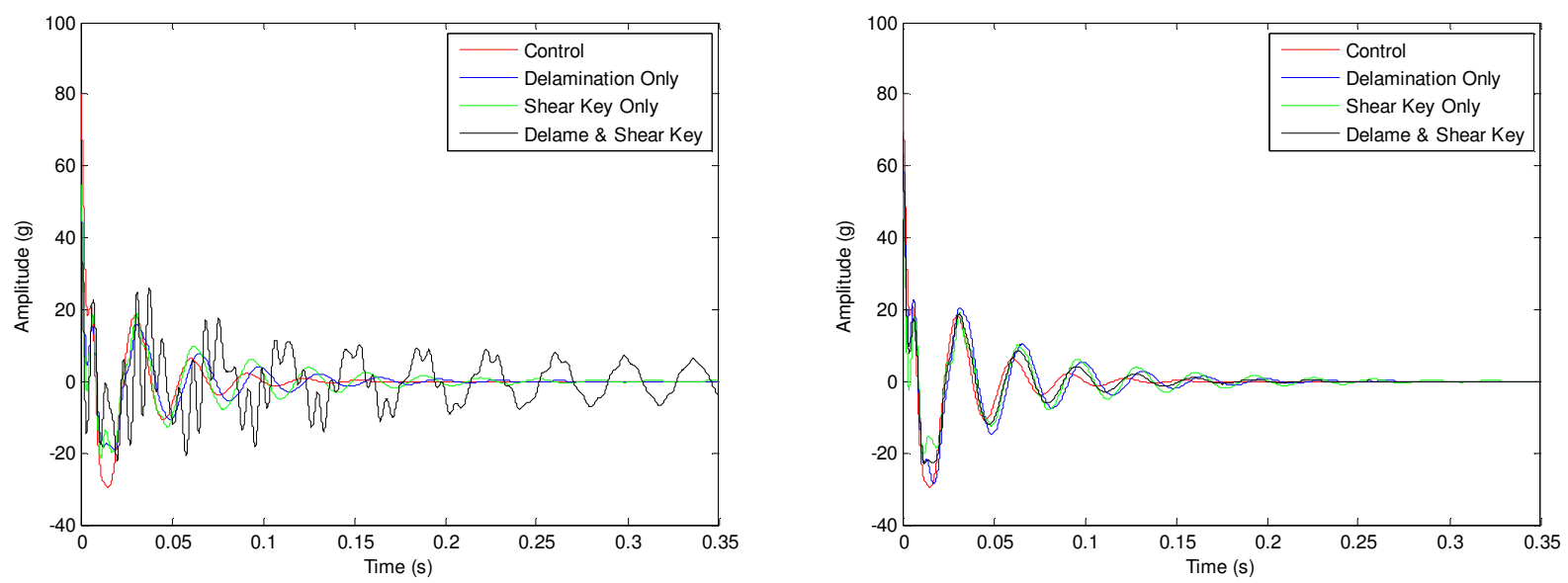

Figure 64: Time response for the carbon fiber plate specimens for the bottom (right) and middle (left) subcases.

The results from the sandwich beam composites show a slight difference when compared to the results from the plate composites. For all three beam cases the results had different values, but similar trends. The shear key specimens with and without delamination showed a faster dampening rate than that of a specimen with delamination. An illustration of this occurrence can be seen on Fig. 65, where the control, shear key-only, and delamination with shear key dampen faster than that of the delaminated specimen. In this middle case study, the delaminated specimen clearly lags behind the remaining specimens over time. From this result and from the other beam case results, applying a shear key into the foam core will assist the dampening response of a delaminated specimen.

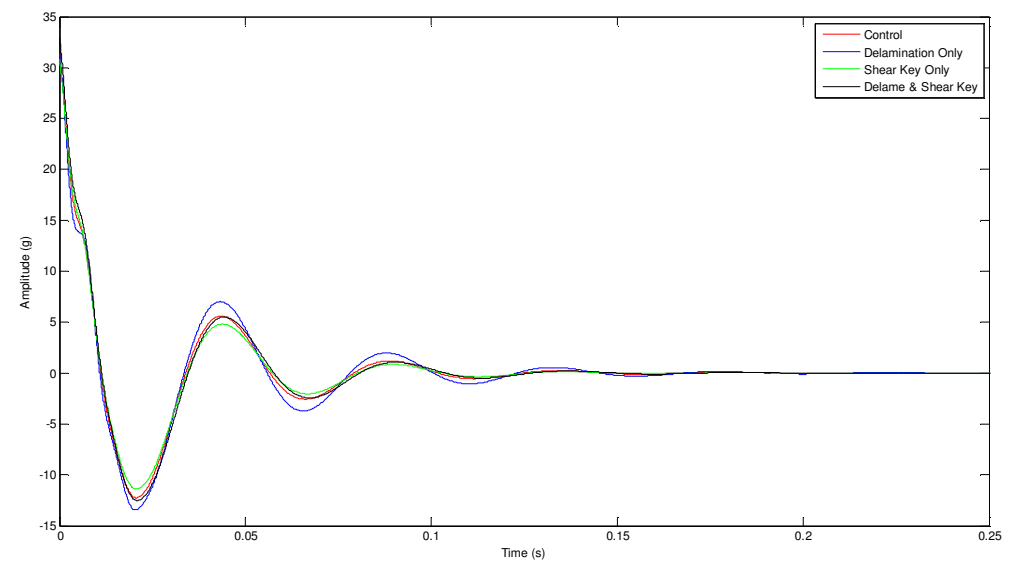

Figure 65: Time response for the middle carbon fiber sandwich beam case.

The final experiment conducted included specimens with shear keys placed vertically along the length of the beam. In this study the specimens were categorized in groups with and without shear keys 
inside the foam core. Figure 66 illustrated the time response of all the specimens conducted in this experiment. It is important to remember that each specimen had different delamination regions that range from 1 inch to 3 inches. The control cases from these graphs illustrate the specimens without any delamination associated with the sample. From the graph, it clearly shows that specimens with shear keys dampen faster than the specimens without shear keys. Along with the delamination specimens there is also a control specimen that has similar attributes as the delaminated cases. This shows that the control case without a shear key also has a slower dampening rate than a composite specimen with delamination and a shear key. The trend line different from the rest is the specimen without shear keys and a delamination of 3 inches between the facesheet and core. This exemplifies the benefit of having vertical shear keys embedded into the structure, which will help the overall dampening rate of the system.

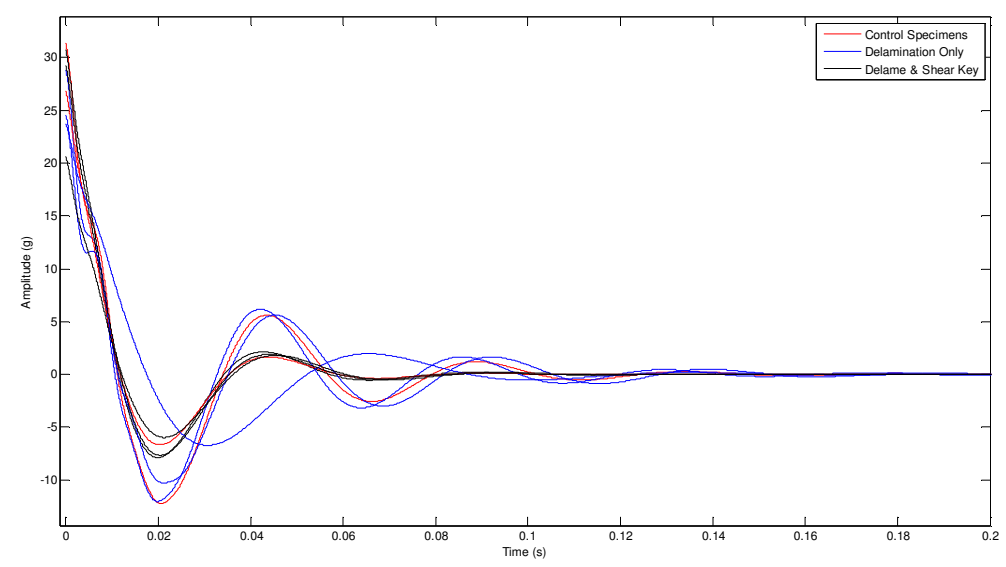

Figure 66: Time response for all the vertical shear cases with different delamination regions and sub-cases

\subsection{Resonance Frequency to Weight Ratio}

When analyzing composite structures, the strength to weight ratio is very important in deciding whether adding material onto a structure to increase the overall strength is beneficial without adding considerable weight. However, when evaluating the vibration response of the structure, it becomes irrelevant. For this reason, the resonance frequency for the first mode will be compared to the weight of the specimen. Table 7 shows the relationship between the weights of the middle case specimens versus the resonance frequency response for the different sub-cases. Even though there are differences between the values of each case study, the results of the frequency to weight ratio have relatively the same results.

Table 7: Comparison between the weight and the resonance frequency for the middle case at the first mode.

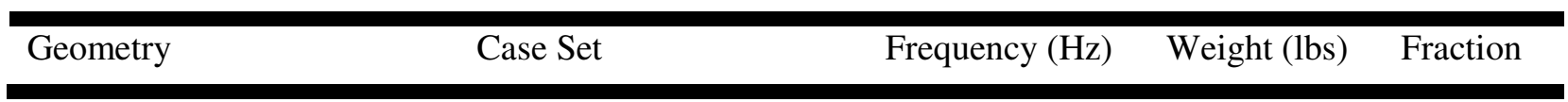




\begin{tabular}{llccc}
\hline Plate & Control & 206.57 & .635 & 325.31 \\
& Delame w/o Shear Key & 191.9 & .636 & 301.73 \\
& Shear Key Only & 200.1 & .655 & 305.49 \\
& Delame with Shear Key & 202.33 & .656 & 308.42 \\
Beam & Control & 142.8 & .0631 & 2263.07 \\
& Delame w/o Shear Key & 141.4 & .064 & 2209.38 \\
& Shear Key Only & 144.01 & .066 & 2181.96 \\
& Delame with Shear Key & 143.42 & .0655 & 2189.62 \\
2-Inch Delaminated Beam & Control & 142.8 & .0631 & 2263.07 \\
& Delame w/o Shear Key & 136.8 & .064 & 2137.5 \\
& Shear Key Only & 146.2 & .075 & 1949.33 \\
3-Inch Delaminated Beam & Delame with Shear Key & 145.8 & .076 & 1918.42 \\
& Delame w/o Shear Key & 96.84 & .0645 & 1501.39 \\
\hline
\end{tabular}

The results from the vibration analysis for the beam and plate cases suggest that the beam case had a higher frequency to weight ratio when compared to the sandwich plate. However, the weight of the shear key is much higher than the weight of the foam core, which caused the fraction to become lower than expected. For the sandwich plate case, the entire structure had a higher weight value, so introducing a fiberglass shear key into the foam would not alter the overall weight of the structure. Therefore, in terms of frequency to weight ratio, the sandwich plate specimens offered better results than a sandwich beam. The only case where there was some benefit in having a shear key inside the structure was in the 3-inch delaminated case. In this case over $30 \%$ of the surface is delaminated on one side of the sandwich facesheet. Only then, and perhaps for specimens with $25 \%$ of a delaminated surface, would embedding a shear key into the foam increase the advantage of having a shear key in terms of resonance frequency to weight ratio. 


\section{Numerical Finite Element Analysis}

Finite element models could be obtained with the assistance of the experimental data collected from the material properties experiments. It is vital to have correct material properties in order to have the numerical values validate the experimental values obtained from the vibration analysis. Cosmos finite element software was utilized to determine the modal response of the plates and the bending mode natural frequencies. Finite element models were generated and meshed using GeoStar pre-processing software. In total, 64 finite element models were analyzed with varying delamination regions and specimens with and without shear keys embedded into the structure. Along with validating the experimental results, the experimental data can also be used to assess the uncertainty of the finite element results.

\subsection{Material Properties Selection for the Finite Element Analysis}

In order to have accurate finite elements results, the material property for the carbon fiber, foam core, and the fiberglass shear keys are required. All the material properties were taken from the experimental static test, except for the Last-A-Foam. The test specimen needed to be cut to a $2 \times 2$ inch square cross-section with a height of 2 inches in order to conduct the test method for obtaining the PVC (polyvinylchloride) foam mechanical characteristics that follow the ASTM D $1621^{5}$ standard. With skewed results when comparing the experimental and manufacturer's values, further investigation was needed to acquire accurate material properties. Once obtained, numerical results were calculated by using the finite element program Cosmos with the pre-software GeoStar to validate the experimental results.

To further investigate the material properties of the LAST-A-Foam Core, a 10 x 10 inch plate was made and then placed on the vibration table to undergo dynamic analysis. The natural frequencies from the vibration table could then be compared to the finite element numerical values, which can be altered by the properties placed on the foam material. Therefore, comparisons between the manufacturer's material properties and the experimental values could be performed by comparing them to the vibration results. However, to ensure and to validate that the values from the vibration table were similar to that of the material being vibrated, a two-layer laminate of carbon fiber was studied on the vibration table.

Knowing that accurate data were obtained for the carbon fiber specimens will ensure that the natural frequencies obtained from the vibration table will be similar to the natural frequencies obtained from the finite element program. Similar procedures were followed for the two-layer $10 \times 10$ inch 
laminate plate to secure the specimen on the vibration table. The results of the data obtained from the vibration table and from the numerical program can be seen in Fig.67.

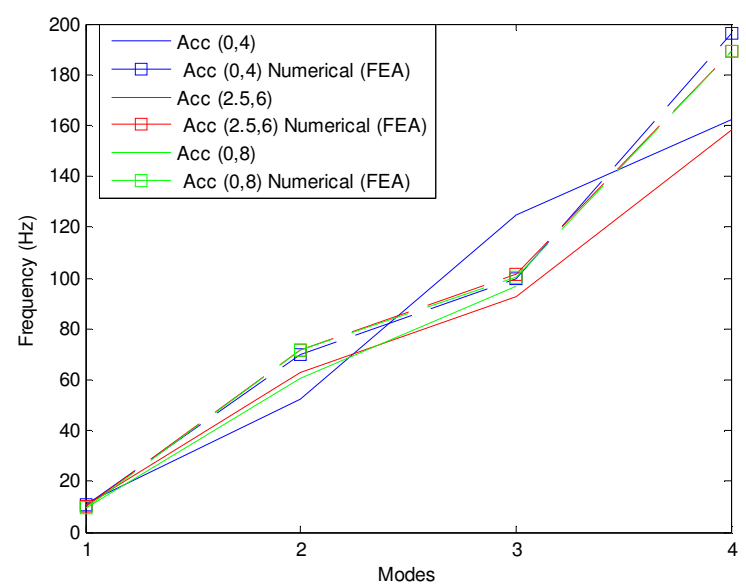

Figure 67: Comparison between the carbon fiber numerical finite element values to the vibration table results.

At first glance it may seem that the data from the experimental analysis does not match with the values from the finite element analysis. However, if only considering when the accelerometer is not placed 4 inches above the aluminum block, then most of the values are consistent up to the third resonance frequency. Unlike the previous experiments, this experiment only had one specimen to obtain data from. If further tests were conducted on the carbon fiber plate, then the frequency response for this specimen with the accelerometer placed 4 inches above the aluminum block, would have been determined as an outlier and not used when averaging the resonances of the carbon fiber. However, the main objective of this experiment was to determine if it was valid to assume that the natural frequencies of the vibration table match the natural frequencies of the finite element models. Table 8 shows the percent error from the numerical and experimental values.

Table 8: Relationship between the numerical and experimental values for the carbon fiber plate specimen.

\begin{tabular}{lllll}
\hline & First Mode & Second Mode & Third Mode & Fourth Mode \\
Experimental Acc $(0,4)$ & 11.44 & 52.19 & 124.8 & 162.3 \\
Theoretical (FEA) & 11.05 & 70.14 & 100 & 196 \\
Error Percentage (\%) & 3.41 & 34.39 & 19.87 & 20.76 \\
Experimental Acc (0,8) & 10.73 & 62.73 & 92.64 & 158.3 \\
Theoretical (FEA) & 10.46 & 71.69 & 101.24 & 189.18 \\
Error Percentage (\%) & 2.51 & 14.28 & 9.28 & 19.51 \\
\hline
\end{tabular}


The first and third modes of the two sets of specimens are very similar to the numerical analysis. However, if considering the second and fourth modes, then the comparison shows that the data from the experimental vibration is not matching with the results of the numerical analysis. When matching values for the foam material, it is important that the first and third modes are similar to each other to have a valid assumption for the material properties. The main cause for this discrepancy could have manifested from not implementing the correct accelerometer weight or criteria onto the mesh of the carbon fiber model. Therefore, the accelerometer's weight and model will be revisited later during this section.

When conducting the foam experiment, three different foam specimens were vibrated and compared to the finite element program COSMOS. In COSMOS the geometry, boundary conditions, and concentrated mass of the accelerometer were set exactly the same for each material property set. The only difference in each model was the material selection that was placed on the foam. The results of the comparisons between the experimental and numerical natural frequencies are shown in Fig. 68. The top black line indicates the natural frequency of the finite element program using the material properties specified by the manufacturer, while the bottom trend line indicates the natural frequency of the experimental material properties. The resonance values for all three specimens were averaged for the first and third modes in order to be compared to each of the finite element values. The error percentage was then calculated for the first and third modes, which were $9.57 \%$ and $14.79 \%$, respectively, for the manufacturer's material properties. The first and third modes had error percent values of $26.58 \%$ and $19.49 \%$, respectively, for the experimental material properties. These percentages were then averaged for each material property set to determine the average error percentage for the entire model.

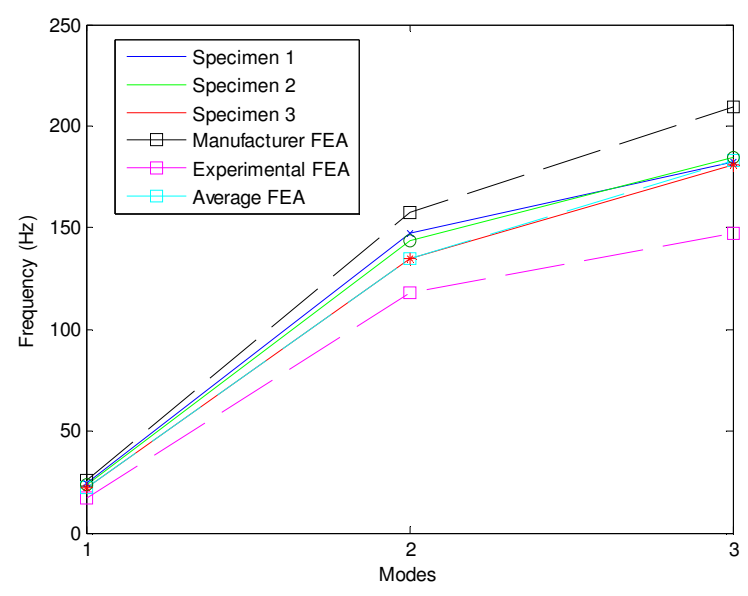

Figure 68: Evaluating the experimental and manufacturing finite element models to the vibrated foam specimens. 
Knowing that the manufacturer's frequency values were higher and the experimental frequency values were lower than the frequencies of the vibrated foam core, Eq. 6 was used to determine the average value for the model.

$$
\frac{\left(M a t_{\text {exp }}+M a t_{\text {exp }} * \text { error }_{\text {exp }}\right)+\left(M a t_{m a}-M a t_{m a} * \text { error }_{m a}\right)}{2}
$$

The expression $M a t_{\text {exp }}$ represents any of the material properties used in the finite element program. The term error $_{\text {exp }}$ is the average error percentage for the experimental property set. The terms with a subscript $\mathrm{m}$ are values used from the manufacturer's material property sets. Therefore, to acquire the necessary material properties for the finite element program the elastic modulus, shear modulus, Poisson's ratio, and density are applied separately to the equation to obtain the average values for the foam. Table 9 shows the list of the material properties for all three cases: manufacturer's, experimental, and average.

Table 9: Manufacturer's, experimental, and average material properties for Last-A-Foam FR 6710.

\begin{tabular}{|c|c|c|c|}
\hline & $\begin{array}{l}\text { Manufacturer's Values } \\
\text { for LAST-A-FOAM FR- } \\
6710\end{array}$ & $\begin{array}{l}\text { Experimental Values for } \\
\text { LAST-A-FOAM FR- } \\
6710\end{array}$ & $\begin{array}{l}\text { Averaged Values for } \\
\text { LAST-A-FOAM FR- } \\
6710\end{array}$ \\
\hline Elastic Modulus_zx(psi) & 19665 & 5074 & 12196.21 \\
\hline Elastic Modulus_xy (psi) & 10052 & 4414.6 & 7385.99 \\
\hline Shear Modulus_zx (psi) & 3195 & 1951.53 & 2767.94 \\
\hline Shear Modulus_xy (psi) & 2823 & 1697.32 & 2358.02 \\
\hline Poisson's ratio & .3 & .3 & .3 \\
\hline Density (lbs./in3) & .00578 & .0059 & .0058 \\
\hline
\end{tabular}

Using the material properties obtained from the static analysis and dynamic analysis, the following values depicted in Table 10 are used during the finite element analysis. The values acquired from the experimental and modal analysis were then changed to fit the units used in the finite element program.

Table 10: Material properties implemented onto the finite element models.

\begin{tabular}{lccc}
\hline Material Properties & LAST-A-FOAM FR-6710 & $\begin{array}{c}\text { Carbon Fiber } \\
\text { (LTM45EM/ CF1803) }\end{array}$ & $\begin{array}{c}\text { Fiberglass Woven } \\
\text { Roving 18oz. }\end{array}$ \\
Density (lbm s2/in4) & .00001508 & .0001544 & .0001503 \\
Elastic Modulus_Ex (psi) & 12196.21 & 7772000 & 2378463 \\
Elastic Modulus_Ey(psi) & 7385.99 & 7772000 & 455862 \\
Elastic Modulus_Ez (psi) & 7385.99 & 7772000 & 455862 \\
Poisson's ratio_vxy & .3 & .044 & .05 \\
\hline
\end{tabular}




\begin{tabular}{lccc}
\hline Poisson's ratio_vxz & .3 & .044 & .56 \\
Poisson's ratio_vzy & .3 & .044 & .56 \\
Shear Modulus_Gxy (psi) & 2767.94 & 372222.22 & 113260.43 \\
Shear Modulus_Gxz (psi) & 2358 & 372222.22 & 146109.6 \\
Shear Modulus_Gzy(psi) & 2358 & 372222.22 & 146109.6 \\
\hline
\end{tabular}

\subsection{Analytical Calculations}

An analytical approach can be used for modal analysis of composite structures. Reddy ${ }^{25}$ outlines the theory for calculating natural frequencies of composite plates in bending. This theory uses the EulerBernoulli formulation of the equations of motion to analyze a single plate with $x-z$ symmetry and can be applied to orthotropic beams or plates.

Important assumptions that were used in this theory are that the laminas of the plate are perfectly bonded to each other and that the plate has perfect symmetry. Additionally, this method only applies to a vibrational response that is primarily bending, which occurs when a structure is much longer than it is wide. The following relations give the equations of motion in terms of displacements. This formulation shows the grand vector matrix of the entire composite structure in equations 7 and 8 .

$$
\begin{aligned}
& \left\{\begin{array}{l}
N_{x x} \\
N_{y y} \\
N_{x y}
\end{array}\right\}=\left[\begin{array}{lll}
A_{11} & A_{12} & A_{16} \\
A_{12} & A_{22} & A_{26} \\
A_{16} & A_{26} & A_{66}
\end{array}\right]\left\{\begin{array}{c}
\frac{\partial u_{0}}{\partial x}+\frac{1}{2}\left(\frac{\partial w_{0}}{\partial x}\right)^{2} \\
\frac{\partial v_{0}}{\partial y}+\frac{1}{2}\left(\frac{\partial w_{0}}{\partial y}\right)^{2} \\
\frac{\partial u_{0}}{\partial y}+\frac{\partial v_{0}}{\partial x}+\frac{\partial w_{0}}{\partial x} \frac{\partial w_{0}}{\partial y}
\end{array}\right\}-\left[\begin{array}{lll}
B_{11} & B_{12} & B_{16} \\
B_{12} & B_{22} & B_{26} \\
B_{16} & B_{26} & B_{66}
\end{array}\right]\left\{\begin{array}{c}
\frac{\partial^{2} w_{0}}{\partial x^{2}} \\
\frac{\partial^{2} w_{0}}{\partial y^{2}} \\
2 \frac{\partial^{2} w_{0}}{\partial x \partial y}
\end{array}\right\} \\
& \left\{\begin{array}{l}
M_{x x} \\
M_{y y} \\
M_{x y}
\end{array}\right\}=\left[\begin{array}{lll}
B_{11} & B_{12} & B_{16} \\
B_{12} & B_{22} & B_{26} \\
B_{16} & B_{26} & B_{66}
\end{array}\right]\left\{\begin{array}{c}
\frac{\partial u_{0}}{\partial x}+\frac{1}{2}\left(\frac{\partial w_{0}}{\partial x}\right)^{2} \\
\frac{\partial v_{0}}{\partial y}+\frac{1}{2}\left(\frac{\partial w_{0}}{\partial y}\right)^{2} \\
\frac{\partial u_{0}}{\partial y}+\frac{\partial v_{0}}{\partial x}+\frac{\partial w_{0}}{\partial x} \frac{\partial w_{0}}{\partial y}
\end{array}\right\}-\left[\begin{array}{lll}
D_{11} & D_{12} & D_{16} \\
D_{12} & D_{22} & D_{26} \\
D_{16} & D_{26} & D_{66}
\end{array}\right]\left\{\begin{array}{c}
\frac{\partial^{2} w_{0}}{\partial x^{2}} \\
\frac{\partial^{2} w_{0}}{\partial y^{2}} \\
2 \frac{\partial^{2} w_{0}}{\partial x \partial y}
\end{array}\right\}
\end{aligned}
$$

Where the A matrix is extensional stiffness, which is

$$
A_{i j}=\sum_{k=1}^{N} \bar{Q}_{i j}^{(k)}\left(z_{k+1}-z_{k}\right)
$$

The B matrix is the bending-extensional coupling stiffness matrix, which is 


$$
B_{i j}=\frac{1}{2} \sum_{k=1}^{N} \bar{Q}_{i j}^{(k)}\left(z_{k+1}^{2}-z_{k}^{2}\right)
$$

And the D matrix is the bending stiffness matrix, which is

$$
D_{i j}=\frac{1}{3} \sum_{k=1}^{N} \bar{Q}_{i j}^{(k)}\left(z_{k+1}^{3}-z_{k}^{3}\right)
$$

Each of the above matrices is a function of the transformed lamina stiffnesses, $\bar{Q}_{i j}^{(k)}$ and the vertical lamina coordinates. The transformed lamina stiffness matrix can be found from the components of the lamina stiffness matrix, $Q_{i j}^{(k)}$, which is a function of material properties.

$$
\begin{gathered}
Q_{11}=\frac{E_{1}}{1-v_{12} v_{21}} \\
Q_{12}=\frac{v_{12} E_{2}}{1-v_{12} v_{21}} \\
Q_{22}=\frac{E_{2}}{1-v_{12} v_{21}} \\
Q_{66}=G_{12}
\end{gathered}
$$

Inverting the D matrix and solving for the differential transverse deflection tensor, this is depicted in Eq. 13. Where the elements $D_{i, j}^{*}$ denote the elements of the inverse of $D_{i, j}$.

$$
\left\{\begin{array}{c}
\frac{\partial^{2} w_{0}}{\partial x^{2}} \\
\frac{\partial^{2} w_{0}}{\partial y^{2}} \\
2 \frac{\partial^{2} w_{0}}{\partial x \partial y}
\end{array}\right\}=-\left[\begin{array}{ccc}
D_{11}^{*} & D_{12}^{*} & D_{16}^{*} \\
D_{12}^{*} & D_{22}^{*} & D_{26}^{*} \\
D_{16}^{*} & D_{26}^{*} & D_{66}^{*}
\end{array}\right]\left\{\begin{array}{l}
M_{x x} \\
M_{y y} \\
M_{x y}
\end{array}\right\}
$$


In order to find the exact solution for natural vibration, it is necessary to solve the Euler-Lagrange equation of motion in terms of displacement. This is possible by combining the transverse deflection equation that is a function of only of the $\mathrm{x}$ coordinate and time with the Euler Lagrange equation of motion for out-of-plane deflection. With various assumptions of the motion of the plate and assuming that the composite plate is symmetrical, Eq. 14 can be used to determine the natural frequency of the structure.

$$
\omega=\sqrt{\lambda^{4}\left(\frac{-b}{\hat{I}_{0} * D_{11}^{*}}\right)\left(\frac{1}{1+\left(\frac{\hat{I}_{2}}{\hat{I}_{0}} \lambda^{2}\right)}\right)}
$$

Again, equation (61) can be used to solve for natural frequencies when the eigenvalues that are appropriate to the boundary conditions. Where $I_{0}, I_{1}$, and $I_{2}$ are the rotary inertias defined as follows.

$$
\left\{\begin{array}{c}
I_{0} \\
I_{1} \\
I_{2}
\end{array}\right\}=\int_{-h / 2}^{h / 2}\left\{\begin{array}{c}
1 \\
z \\
z^{2}
\end{array}\right\} \rho_{0} d z
$$

All of these constants are dependent on $\lambda$ and the length of the beam. The value of $\lambda$, which correlates to the natural frequency of the structure, satisfies the characteristic equation for its particular boundary condition. The characteristic equations and their eigenvalues are presented below in eq. 11 . This equation is the representation of a fixed free boundary condition of the composite sandwich specimen.

$$
\lambda=\frac{1.875}{a}, \frac{4.694}{a}
$$

\subsection{Sandwich Plate Finite Element Models}

In order to validate the experimental results obtained from the experimental procedures, various finite element models were created to compare the numerical and experimental results. The finite element model used for this research was the pre-software GeoStar and the post-software COSMOS. Although COSMOS is not the best program available for creating accurate representations of composite materials, it was the only program accessible throughout the entire research. Nonetheless, all the different case studies and their respective sub-cases were all created in order to validate the experimental results. 


\subsubsection{Constructing the Control Finite Element Model}

With the measurements of the carbon fiber facesheet known, the cross-sectional area of the plate can be recreated in GeoStar. To create geometry in a three dimensional field on GeoStar, geometry drop down menu on the top screen was first selected. This menu will displace different geometries such as points, curves, surfaces, etc. The option "points" was selected and the carbon fiber facesheet geometry was defined by the $\mathrm{x}, \mathrm{y}$, and $\mathrm{z}$ coordinate system. Once the points are created, surfaces are then formed by selecting the previous point in a counterclockwise routine. By creating a surface, the program will automatically create curves for each side of the surface that was just created. To extrude the cross sectional area, volume from the geometry pull down menu was selected. After selecting volume, the value placed in the $\mathrm{x}$-direction was 0.025 inches, which generated one side of the carbon fiber facesheet. The next volume extruded was the foam core which followed the previous process. However, point or surfaces for the foam are not required for the model. By extruding the surface of the carbon fiber facesheet into a volume, GeoStar automatically created surfaces for this new volume. Therefore, to create the foam core, the adjacent surface of the carbon facesheet is selected, and then extruded to create the core volume. The value recorded during the extraction, in the x-direction, was .5 inches. Similarly, the opposing carbon fiber facesheet was created by the foam core's surface. Instead of selecting the first facesheet surface, the adjacent core surface was selected to create the volume. The value that was implemented into the model was 0.025 inches to make the model symmetrical.

The element group was then defined as a solid geometry to create the mesh for the volumetric model. The solid element was the element choice for the plate model in order to capture the semicircle geometry of the shear keys. This would not be possible for quadrilateral element or any other hyper elements that are used for 2-D analysis. However, for this experimental hybrid elements or any other type of formulation element were not considered; even thought the different element forms could decrease the error percentage between experimental and numerical. To implement material properties into the model, the scroll down icon "Propset" on the top screen was selected. Using Table 10, all the carbon fiber properties were introduced into the model. The carbon fiber mesh was constructed prior to the foam core material properties. An important aspect in creating a mesh, is to make sure all the nodes in the entire model are aligned in order to receive results. If the nodes are not properly aligned, the model will not be fully connected and will detach when analyzed by the solver. The volumetric mesh consisted of 80 elements for both the length and height of the facesheet, but the thickness consisted of two elements. Two elements were selected in order to have one per lamina of carbon fiber. The next process was to add the material properties for the foam core. Using Table 9, all the material properties were implemented into the 
program. Once again the volumetric mesh for the foam core consisted of 80 elements for the length and height, but the thickness consisted of 10 elements. The scope of this analysis concentrated on the interaction between the foam and carbon fiber facesheets when delamination occurred. Therefore, it was unnecessary to have a high fidelity in the core structure of the model. Once the mesh is created, the node option to merge all nodes in a given tolerance is selected. Merging the nodes will force any nodes that are directly on top of each other to fuse together and act as one node. This made the entire structure connected by the nodes. These nodes were also compressed to reduce the time of the analysis process.

The boundary conditions and forces that are acting on the composite plate are then selected for the model. The gravitational effects are also added onto the model in the y-direction. In GeoStar, there is an icon that could be selected in order to apply a gravitational field onto the model. This field was specified and applied on the top portion of the plate and beam composite sandwiches. In the same toolbar section, the boundary conditions of the carbon fiber sandwich are identified. In this case, the plate is fully fixed on the bottom surface of the plate. Along with the bottom surface being selected, parts of the two carbon fiber facesheets are also fully fixed. Due to the vibration fixture, the bottom one inch of the sandwich composite is secured by two aluminum blocks. The sandwich composite is compressed by these two aluminum plates in order to fix the composite plate into the vibration table. The result of this compression will cause the two facesheets that are in contact with the aluminum block to be restricted in all degrees of freedom. Therefore, restricting the $1 \times 10$ inch region of the nodes outside the surface of both carbon fiber facesheets are only taken into consideration. The inside foam or the remaining carbon fiber nodes are not confined to this boundary condition. Once the boundary conditions and loads are set, the software's analysis program can commence by selecting the frequency analysis. Figure 69 shows the results of the FEA model of the deformed shape and the deflection of the composite structure. From the results, the resonance values were obtained to compare them to the experimental values.
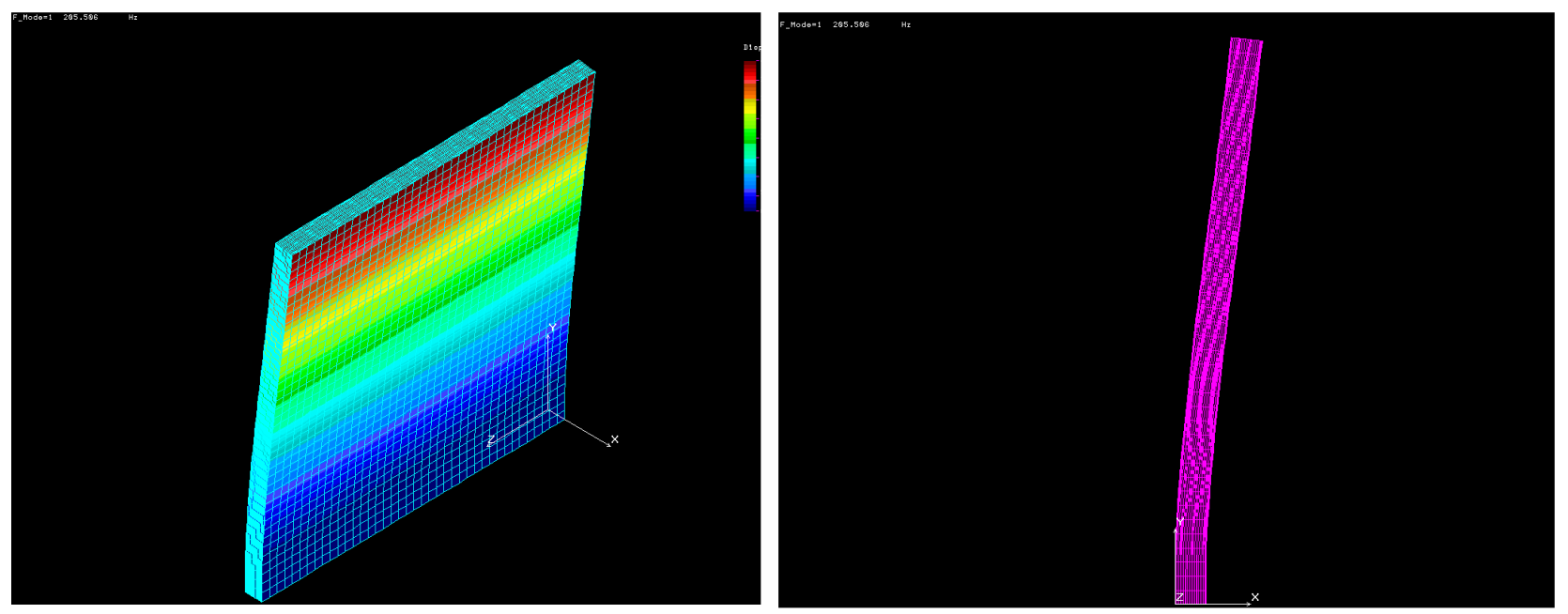
Figure 69: Finite element model of the control plate using GeoStar and COSMOS.

To fully understand whether the composite sandwich plate has fully converted to a finite value, further studies were performed on the mesh and elements. The finite element model was revaluated again, but with fewer nodes and elements. As previously described, the model had a total of 80 elements per each edge of the composite sandwich plate. For this iteration the number of elements applied to edge of the model will be increased in increments of 20 elements per each side, until the total number of elements has reached 80 elements per each side. Figure 70 shows the convergence study for the control composite sandwich plate. This graph shows that even with 20 elements per side, the value is still reasonable when compared to more element values. Nonetheless, this shows that the 80 element model for the composite sandwich finite element model will provide converged results.

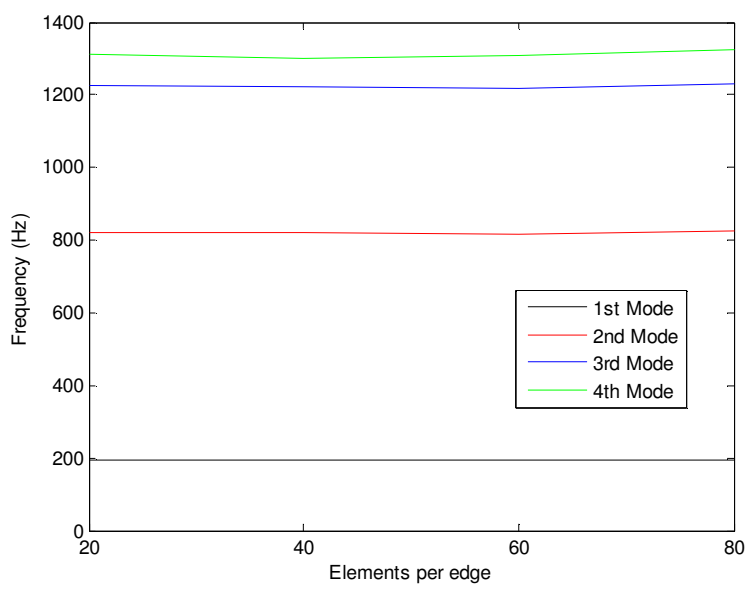

Figure 70: Illustration of the convergence study for the control composite sandwich plate.

For the experimental analysis, there were three control specimens that were vibrated during the experiment. From these three composite plates, an average was taken in order to compare the theoretical and numerical resonance values. Table 11 shows the comparison between the theoretical values obtained from the FEA results, the experimental values, and analytical values for the carbon fiber sandwich. From the table, it is easily noticeable that there were several similarities between the numerical and experimental resonance values. The percent error recorded for this experiment ranged from $0.49 \%$ to $15.51 \%$ for the resonance values. The first, second, and fourth modes have the least amount of error, but the third mode is unlike the rest of the modes. When comparing the analytical with the numerical results, there was a closer resemblance in the values. The assumption made for analytical calculation was that the frequency values obtained were for pure bending motion. Therefore, only the first and third modes were compared. The percent error recorded for among the numerical and analytical values ranged from $0.09 \%$ 
to $3.75 \%$ for the resonance values. Unlike the experimental results, the third mode for the analytical analysis is very similar to the finite element model. If comparisons were made between the experimental and analytical analysis, the error percentage for the third mode would be around $16.66 \%$. However, these results conclude that there are similar results between the numerical, experimental, and analytical resonance values for the control specimen.

\begin{tabular}{lllll} 
Table 11: Error percentage between the experimental and numerical results for the control sandwich plate \\
\hline & First Mode & Second Mode & Third Mode & Fourth Mode \\
Experimental Average & 211.5 & 828.13 & 1066.66 & 1378.66 \\
Numerical (FEA) & 205 & 824 & 1232 & 1324 \\
Error Percentage (\%) & 3.07 & .49 & 15.51 & 3.96 \\
Analytical & 205.2 & $\mathrm{NaN}$ & 1280 & $\mathrm{NaN}$ \\
Error Percentage (\%) & .09 & $\mathrm{NaN}$ & 3.75 & 3.96 \\
\hline
\end{tabular}

\subsubsection{Constructing the Top Delaminated Finite Element Model Case}

With the measurements of the carbon fiber facesheet known for the top delaminated sandwich composite, the cross-sectional area of the plate can be recreated in GeoStar. The process for creating one side of the carbon fiber facesheet and the foam core followed the exact process as when making the control composite. However, the opposing carbon fiber facesheet was created differently in order to introduce a delaminated region. Instead of selecting the adjacent core surface, extra surfaces were created in order to extrude the delaminated region separately. Therefore, to complete the area of the carbon fiber facesheet three surfaces had to be made. The first surface had a length of 8.75 inches and a height of 10 inches. The delaminated region had a length of 1 inch and a height of 10 inches. The final surface had a length of 1.25 inches and a height of 10 inches. These three surfaces were all extruded to a value of 0.025 inches to complete the plate model.

The element group was then defined as a solid geometry to create the mesh for the volumetric model. All the carbon fiber properties were introduced to the model by using Table 10. Instead of adding the foam core material properties, the carbon fiber mesh was previously constructed. The nondelaminated and delaminated carbon fiber facesheets had a volumetric mesh consisting of 80 elements for both the length and height of the facesheet, but the thickness consisted of two elements. Two elements were selected in order to have one per lamina of carbon fiber. The length value of the mesh was determined based on the length of the surface. In order to align the nodes for the entire model, the mesh had to have a uniform length to receive accurate results. From the mesh on the non-delaminated facesheet, the mesh corresponded to 80 elements per 10 inches. This implied that there were 8 elements per inch or a 
node placed every 0.125 inches for the model. Therefore, the length of each volume was divided by 0.125 to determine the value for the mesh in this direction. This was done to all three volumes of the delaminated carbon fiber facesheet. Before adding the mesh on the foam core, the nodes for the carbon fiber facesheets were merged. This ensured that the delaminated facesheet would act as a single piece instead of three separate volumes. The next process was to add the material properties for the foam core. Using Table 10, all the material properties were implemented into the program. Once again the volumetric mesh for the foam core consisted of 80 elements for the length and height, but the thickness consisted of 10 elements.

Applying the delamination into the finite element model implied that the nodes at the delaminated region must not be merged together. However, the rest of the foam core needed to be merged with the non-delaminated regions of both facesheets. For this process, the nodes of the foam core were needed for each corresponding delamination. When merging nodes together, the lower order of the nodes would merge with higher order nodes. Therefore, if the first and last node of the delaminated region is known for the foam core then nodes can be merged from one up to the value of the node before the first node on the delaminated region on the core. Once merging was complete, the rest of the nodes were merged, but attaching with the node before the last node on the delaminated region. An important aspect of this model is that the node increases in increments of one on the y-axis. This implies that the length of the delamination occurs on the $y$-axis and the width lies on the $x$-axis. This node orientation also makes it possible to do the node merging for this model.

The boundary conditions and forces that are acting on the composite plate are selected for the model. The gravitational effects are also added onto the model in the z-direction. In the same toolbar section, the boundary conditions of the carbon fiber sandwich are identified. In this case, the plate is restricted in all degrees of freedom on the bottom surface of the plate. In this configuration the bottom of the plate lies on the $x-y$ plane instead of the $y-z$ plane to match the control model. Along with the bottom surface being selected, parts of the two carbon fiber facesheets are also fully fixed. Only the nodes of the $1 \times 10$ inch region on the outside surface of both carbon fiber facesheets were restricted. The inside foam or the remaining carbon fiber nodes are not confined to this boundary condition. Once the boundary conditions and loads are set, the software's analysis program can commence by selecting frequency analysis. Figure 71 shows the results of the FEA model of the deformed shape and deflection of the composite structure. From the results, the resonance values are obtained to compare to the experimental values. 

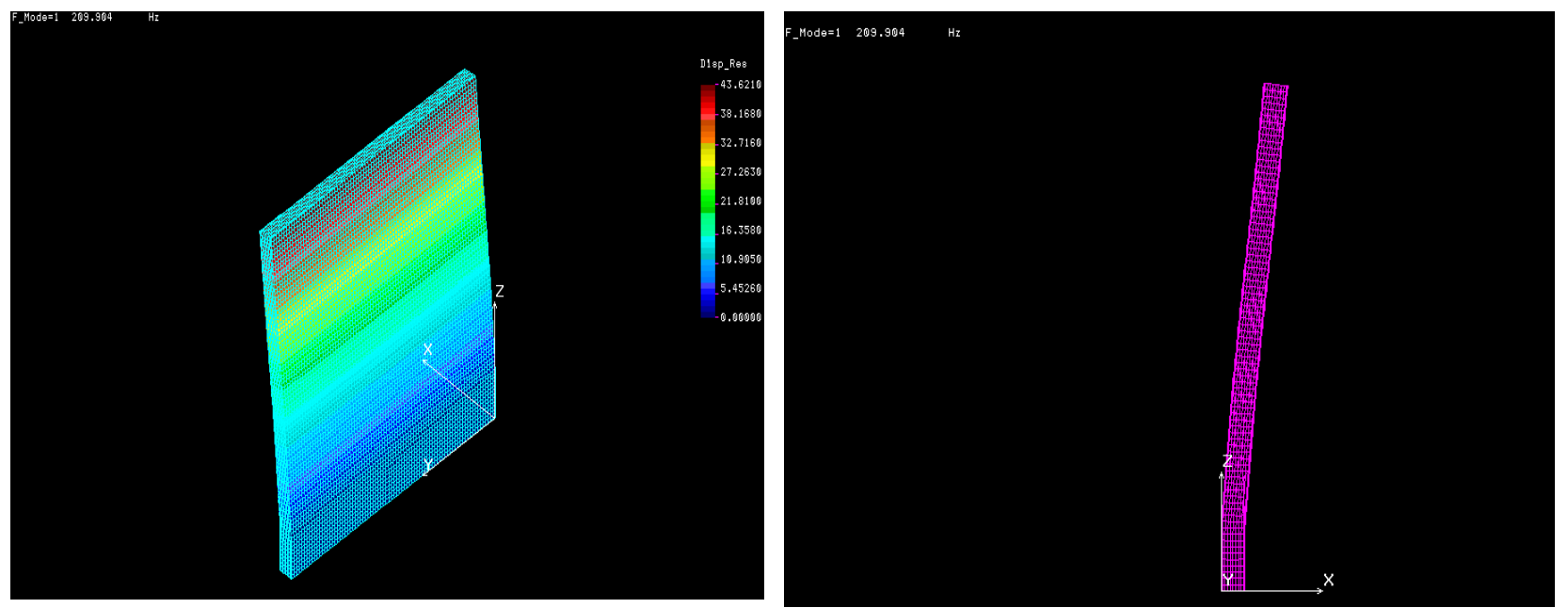

Figure 71: Finite element model of the top delaminated plate created on the pre-software GeoStar.

For the experimental analysis there were five different orientations of the delamination region that were vibrated. The same method was applied to each model, but the delamination location was shifted to fit the measurements of the manufactured specimen. Table 12 shows the comparison between all the finite element models that were produced. From the table, it is easily noticeable that there were close similarities between the different cases and their resonance values. The only specimen that was different from the rest of the modes was the top delamination case. In this case, both the first and third modes were much higher than the other cases. The rest of the models have similar results with slight differences in the values, which was expected because the experimental results were not exactly the same for each case.

Table 12: Finite element results for the first four modes that were detected by the accelerometer for each delaminated-only case study.

\begin{tabular}{lllll}
\hline & First Mode & Second Mode & Third Mode & Fourth Mode \\
Bottom & 185.04 & 774.11 & 1165.59 & 1493.53 \\
Middle & 188.199 & 827.85 & 1123.1 & 1456.63 \\
Top & 203.21 & 792.54 & 1275.79 & 1502.04 \\
Vertical Side & 189.45 & 802.34 & 1154.01 & 1538.58 \\
Vertical Middle & 187.75 & 805.35 & 1155.68 & 1306.38 \\
\hline
\end{tabular}

\subsubsection{Constructing the Bottom Shear Key Finite Element Model Case}

With the measurements of the carbon fiber facesheet known for the bottom shear key sandwich composite, the cross-sectional area of the plate can be recreated in GeoStar. Various changes were made 
to develop the geometry in order to introduce the two shear keys into the foam region at the bottom of the sandwich composite. Instead of extruding each component of the composite in the $\mathrm{z}$ direction as the previous examples, the side that shows the shear key ends, foam, and carbon fiber facesheets were extruded to 10 inches. Before each of the composite components can be extruded, the geometry needed to be made. Depending on the orientation of the shear keys, the foam core was sectioned into five pieces. Three pieces of the foam had no shear keys in the geometry, while the other composed of various sections. This is illustrated in Fig. 72 to give a better representation of the geometry for the bottom shear key-only plate specimen. In order to have the node connect throughout the model, different sections were made where the shear keys were embedded into the foam core.

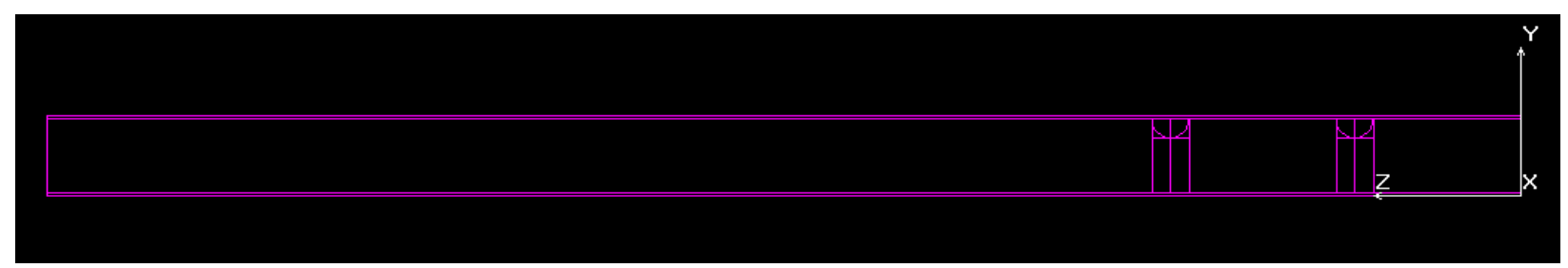

Figure 72: Representation of the finite element geometry for shear keys embedded into the foam.

Using Table 10, all the carbon fiber properties were first introduced to the model. Instead of adding the foam core material properties, the carbon fiber mesh was constructed beforehand. The carbon facesheets had a volumetric mesh consisting of 80 elements for both the length and height of the facesheet, while the thickness consisted of two elements. Two elements were selected in order to have one per lamina of carbon fiber. The length value of the mesh was determined based on the length of the surface. In order to align the nodes for the entire model, the mesh had to have a uniform length to receive accurate results. The next process was to add the material properties for the foam core. Using table 10, all the material properties were implemented into the program. The length of each foam volume was divided by 0.125 to determine the value for the mesh in this direction. This was done to all three volumes of the foam core without a shear key embedded into the core. The following rectangular foam volumes were then meshed to correspond to the following nodes on the facesheets. Under the shear keys were two triangular volumes that consisted of foam properties. These volumes would be matching the nodes that corresponded to the shear key mode.

In order to have accurate results for the models with shear keys, the fiberglass shear keys needed to be meshed correctly. The material properties of fiberglass were placed into the program before meshing the shear keys. For each quarter circle of the shear key, the edge of the circular portion contained 5 nodes, while the other edges had 1 node each. When meshed, elements would all connect towards the center of 
the semicircle shear key. Once the shear keys were meshed, the foam properties were once again activated and the remaining triangular elements were matched to the shear key models.

No delamination was required for this model. When merging nodes together, the lower order of the nodes merged with the high ordered nodes. The same boundary conditions were applied to the model and the frequency analysis was selected to acquire the resonance frequency of the model. Figure 73 shows the results of the FEA model of the deformed shape and the deflection of the composite structure. From the results, the resonance values are obtained to compare to the experimental values. The illustration shows the first and second modes on top and the third and fourth modes on the bottom. One important aspect of the chosen modes is their comparison to the experimental results. The accelerometer used in the experiment is not able to detect pure torsion, while the experiment is running. Therefore, models had to be carefully selected in order to acquire the correct model for each case study.
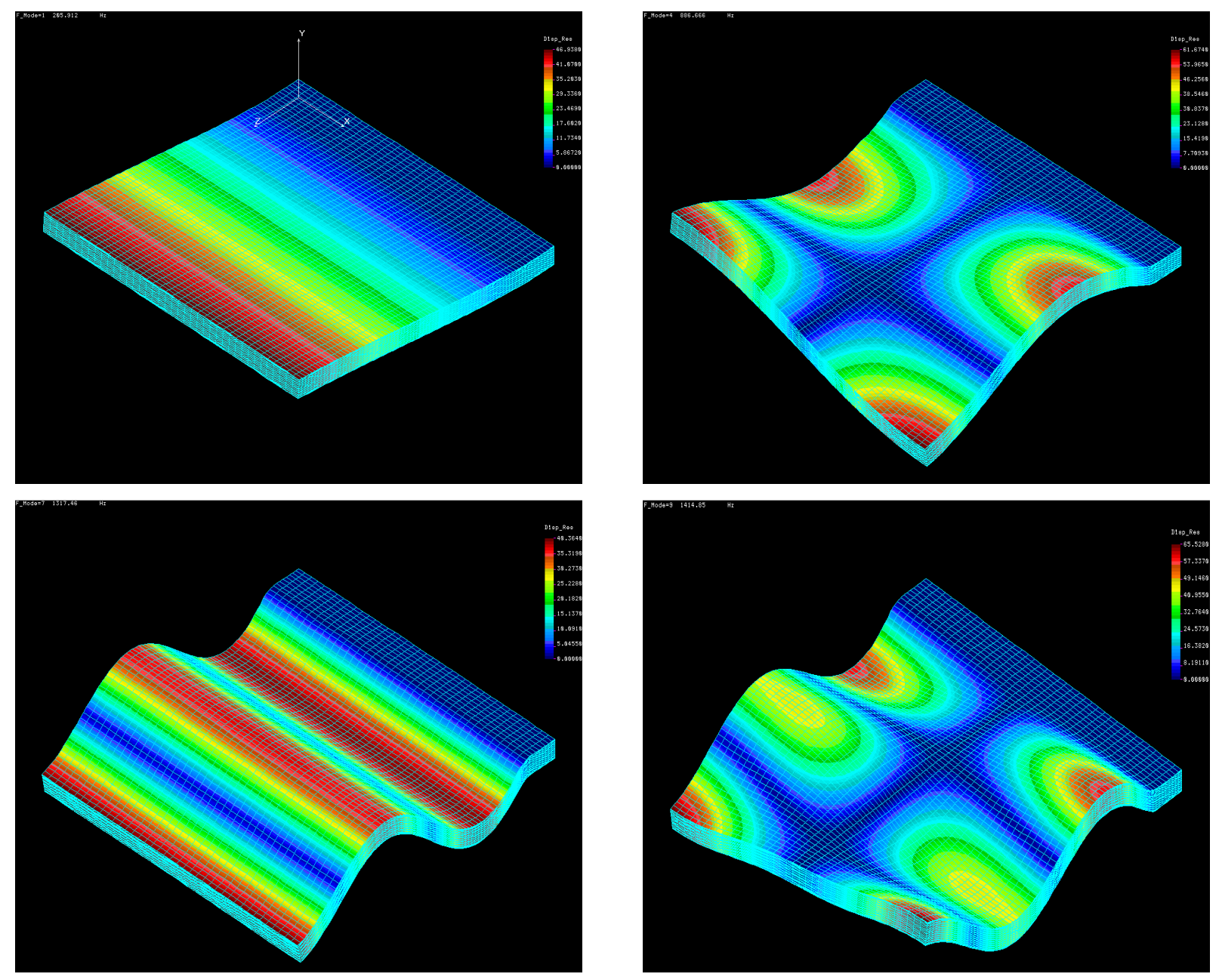

Figure 73: Finite element models of the bottom delaminated case using GeoStar and COSMOS to compare the modes detected by the accelerometer. 
For the experimental analysis there were five different orientations of the delamination region that were vibrated. The same method was applied to each model, but the delamination location was shifted to fit the measurements of the manufactured specimen. Table 13 shows the comparison between all the finite element models that were produced. From the table it is easily noticeable that there were close similarities between the different cases and their resonance values. The models have similar results with slight differences, which was expected because the experimental results were not exactly the same for each case.

Table 13: Finite element results for the first four modes that were detected by the accelerometer for each shear key-only case study.

\begin{tabular}{lllll}
\hline & First Mode & Second Mode & Third Mode & Fourth Mode \\
Bottom & 205.91 & 886.66 & 1317.46 & 1414.85 \\
Middle & 202.48 & 856.2 & 1328.41 & 1424.52 \\
Top & 192.42 & 854.42 & 1302.82 & 1400.2 \\
Vertical Side & 199.87 & 857.05 & 1300.9 & 1393.43 \\
Vertical Middle & 199.99 & 882.04 & 1305.49 & 1420.79 \\
\hline
\end{tabular}

\subsubsection{Constructing the Middle Delaminated Finite Element Model with Shear Keys Case}

Similar to the shear key-only model, the combination of both shear key and delamination had the same geometry and mesh except for the merging of the nodes. The geometry of the middle delaminated plate with shear keys was applied to the model. Meshing the model followed the same steps as in the previous example with the shear key-only model. The element group was then defined as a solid geometry to create the mesh for the volumetric model. The carbon fiber properties used to create the FEA model came from Table 10. The carbon facesheets had a volumetric mesh consisting of 80 elements for both the length and height of the facesheet. The delaminated foam core between the shear keys were applied last to ensure that a delamination occurred in the structure. The core and the shear keys were meshed the same way as aforementioned. The nodes were merged together to connect each component of the structure prior to applying the section of the foam with the delamination.

When merging nodes together, the lower order nodes would need to be merged to the high order nodes. Therefore, if the first and last node of the delaminated region is known for the foam then the nodes can be merged from one up to the value of the node before the first node on the delaminated region. The mesh of the delaminated foam was built to ensure that the other edges of the volume connected to the foam and carbon fiber facesheet. 
The boundary conditions had the same constraints as the previous models due to the fact that all the specimens were placed in the same fixture in the experimental analysis. However, it is very important to have a consistent one inch fully constrained on the sides of the carbon fiber. Failing to do this would alter the values for all the specimens by a slight percent difference of $1.34 \%$. This value was obtained by not constraining the entire one inch, but constraining only 0.8 inches of the model. Even though the sensitivity of the boundary conditions is not catastrophic, it is accurate to have the same boundary conditions throughout the entire numerical analysis. Figure 74 shows the results of the FEA model of the mode shape of the composite structure. From the results the resonance values are obtained to compare the experimental values.
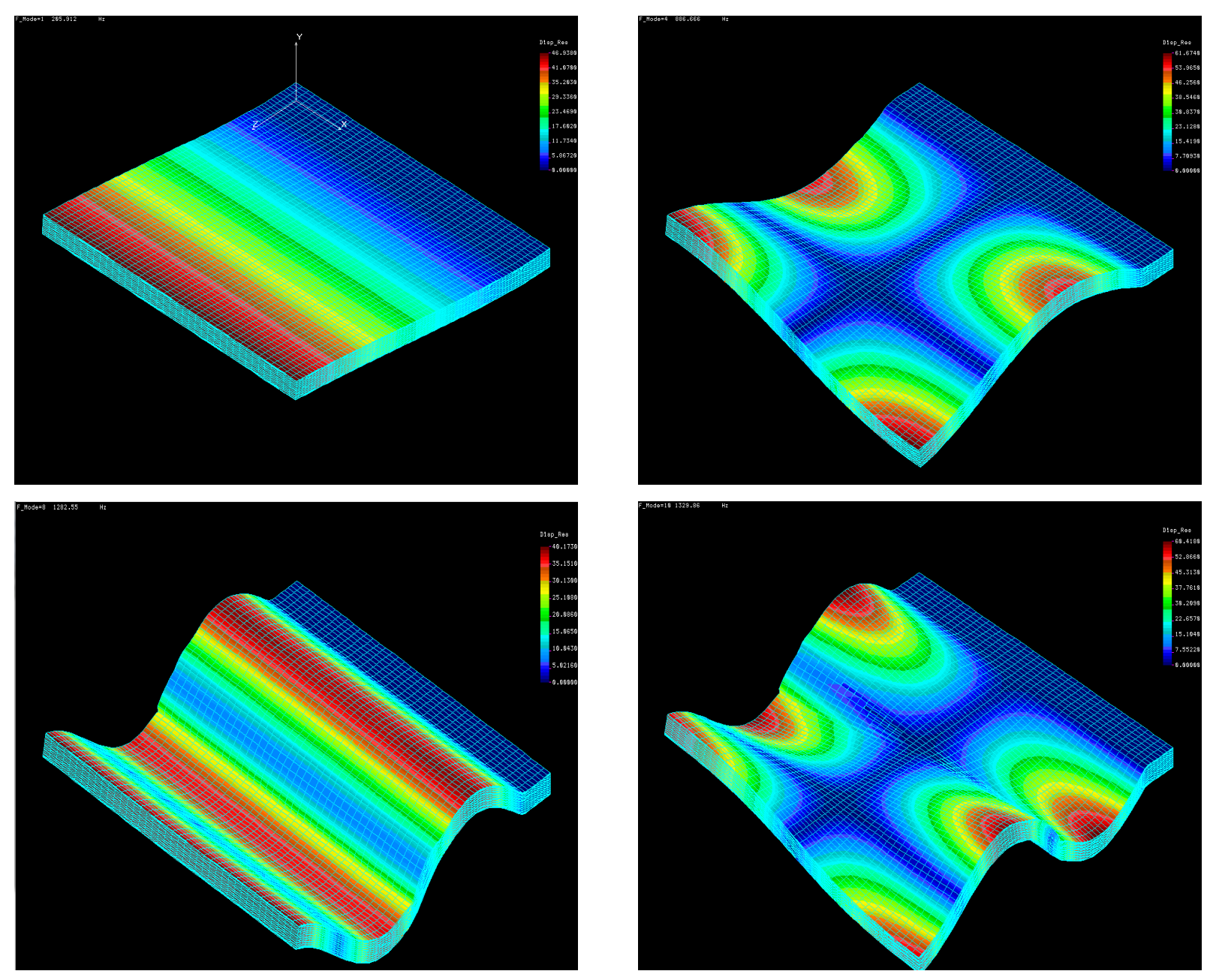

Figure 74: Finite element models of the middle delaminated with shear keys case using GeoStar and COSMOS to compare the modes detected by the accelerometer. 
For the experimental analysis, only five different orientations of the delamination region were vibrated. The same method was applied to each model, but the delamination location was shifted to fit the measurements of the manufactured specimen. Table 14 shows the comparison between all the finite element models that were produced. From the table, it is easy to notice the similarities between the different cases and their resonance values. The only specimen that was different at every mode was the top case. In this case, both the first and second modes were much lower than the other cases. The rest of the models are similar except for slight differences because the experimental results were not equivalent for each case.

Table 14: The Finite element results of the first four modes that were detected by the accelerometer for each delamination with shear key case study.

\begin{tabular}{lllll}
\hline & First Mode & Second Mode & Third Mode & Fourth Mode \\
Bottom & 202.33 & 735.13 & 1276.5 & 1323.7 \\
Middle & 200.27 & 725.259 & 1282.55 & 1329.86 \\
Top & 191.83 & 665.25 & 1284.6 & 1376.38 \\
Vertical Side & 197.417 & 836.36 & 1235.02 & 1370.9 \\
Vertical Middle & 198.13 & 751.368 & 1270.26 & 1368.31 \\
\hline
\end{tabular}

\subsection{Sandwich Beam Finite Element Models}

The second portion of the research was conducting vibration analysis on composite beam geometries. These sandwich composites consisted of a shear key embedded along the length and width of the beam specimen. The specimens that consisted of longitudinal shear key and a delamination region in the center of the composite beam, is increased by increments of 1-inch to determine the effects of a larger delamination region on the natural frequency of the structure. The specimens that had two shear keys embedded widthwise on the beam followed the orientation of the plate specimens, for the middle, bottom, and top case studies. To validate the experimental results obtained from the vibration table, various finite element models were created to compare the numerical and experimental results.

\subsubsection{Constructing the Control Finite Element Model}

The cross-sectional area of the beam can be created in GeoStar by knowing the measurements of the carbon fiber facesheet for the control sandwich composite beam. The process in creating one side of the carbon fiber facesheet and foam core followed the same procedures as aforementioned. After creating 
the carbon fiber facesheet, the value placed in the x-direction was 0.025 inches, which generated one side of the carbon fiber facesheet. The next volume extruded was the foam core, which followed the same process. However, point or surfaces for the foam were not required in the model. By extruding the surface of the carbon fiber facesheet into a volume, GeoStar automatically created surfaces for this new volume. Therefore, to create the foam core, the adjacent surface of the carbon facesheet was selected and extruded to create the core volume. The value recorded during the extraction, in the x-direction, was 0.5 inches. Similarly, the opposing carbon fiber facesheet was created by the foam core's surface. Instead of selecting the first facesheet surface, the adjacent core surface was selected to create the volume. The value implemented into the model was 0.025 inches to make it symmetric.

The element group was defined as a solid geometry in order to create the mesh for the volumetric model. The solid element was the element choice for the beam model in order to capture the semicircle geometry of the shear keys. To implement the material properties into the model, the scroll down icon Propset on the top screen was selected. Table 10 was used to implement the carbon fiber properties for the model. Instead of adding the foam core material properties, the previous carbon fiber mesh was constructed. An important aspect to consider when creating a mesh is to make sure that the nodes in the entire model are aligned; otherwise the solver will not work or the model will be detached. The volumetric mesh consisted of 80 elements for the length and 8 elements for the width of the facesheet, but the thickness consisted of two elements. Two elements were selected in order to have one per lamina of carbon fiber. The next process was to add the material properties for the foam core. The volumetric mesh for the foam core consisted of 80 elements for the length and 8 elements for the width of the foam, while the thickness consisted of 10 elements. The scope of this analysis concentrated on the interaction between the foam and carbon fiber facesheets when delamination occurs. Therefore, it was unnecessary to have a high fidelity in the core structure of the model. Once the mesh is made, the node option to merge all nodes in a given tolerance was made. By merging the nodes it will force any nodes that are directly on top of each other to be fused together and act as one node. This ensured that the entire structure was connected by the nodes. These nodes were also compressed to speed the analysis process when it was run.

The boundary conditions and forces that are acting on the composite beam are then selected for the model. The gravitational effects are also added onto the model in the y-direction. In the same toolbar section, the boundary conditions of the carbon fiber sandwich are identified. In this case, the beam is fully fixed on the bottom surface of the beam. Along with the bottom surface being selected, parts of the two carbon fiber facesheets were restricted in all six degrees of freedom. Due to the vibration fixture, the bottom one inch of the sandwich composite is secured by two aluminum blocks. The sandwich composite is compressed by these two aluminum beams in order to fix the composite beam into the vibration table. The result of this compression will cause the two facesheets that are in contact with the aluminum block to be restricted in all degrees of freedom. Therefore, when restricting the $1 \times 10$ inch region, the nodes of 
the outside surface of both carbon fiber facesheets are only taken into consideration. The inside foam, or the remaining carbon fiber nodes are not confined to this boundary condition. Once the boundary conditions and loads are set, the software's analysis program can commence by running the frequency analysis. However, the resonance values obtained from this numerical analysis were not similar to the experimental results from the vibration experiment. Therefore, further considerations were needed for the finite element beam cases.

Knowing that the weight of the accelerometer was $0.028 \mathrm{lbs}$, this could have easily altered the natural frequency of the specimens without an accelerometer. Therefore, the FEA model for this experiment included the accelerometer acting on the facesheet of the composite. This mass force was applied by replicating the accelerometer onto the beam; four inches from the base of the free plate and directly in the center of the beam. The material properties of the accelerometer consisted of metallic and piezoelectric crystal materials. Therefore the material of the accelerometer was assumed to be a steel alloy with a density of $.00016 \mathrm{lbm}$ s2/in4. Figure 75 shows the results of the FEA model of the control specimens with and without an accelerometer placed on the composite structure. The numerical results of the resonance values obtained from COSMOS are compared to the experimental values.
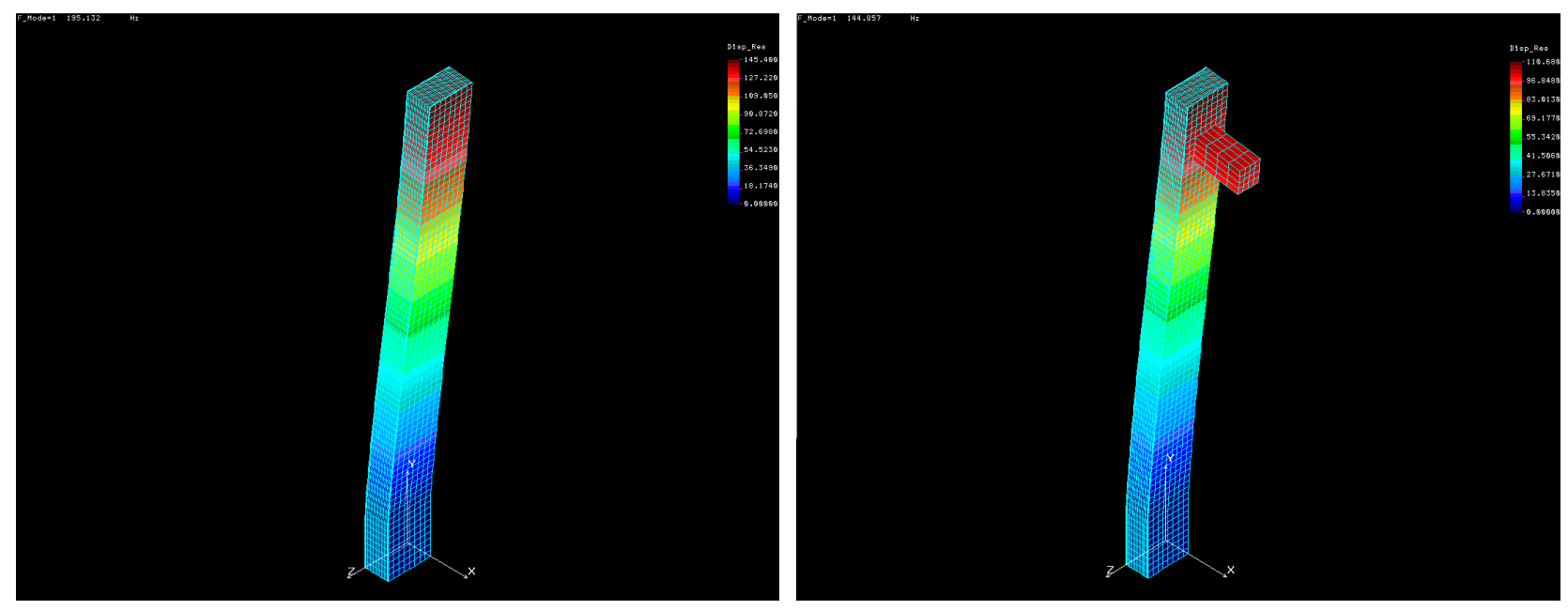

Figure 75: Finite element models of the control beam with (right) and without (left) the accelerometer using GeoStar and COSMOS.

There were seven control specimens for the experimental analysis that were vibrated. From these three composite plates, an average was taken in order to compare the theoretical and numerical resonance values. Table 15 shows the comparison between the theoretical values obtained from the FEA results and the experimental values for the carbon fiber sandwich beam. From the table, it is easily noticeable that the experimental and numerical results from the FEA model with the accelerometer were similar, while the experimental and numerical results from the FEA model without the accelerometer were different. The 
percent error recorded for this experiment ranged from $1.44 \%$ to $6.85 \%$ for the resonance values with the accelerometer, while the error percentage between the experimental and the numerical values without the accelerometer ranged from $19.07 \%$ to $36.65 \%$. These results indicate that the numerical values without the accelerometer are inconsistent with the experimental values. However, these results show similarities between the numerical resonance values modeled with the accelerometer and experimental resonance values for the composite beam specimens.

Table 15: Error percentage between the finite element results with and without the accelerometer place on the control sandwich beam.

\begin{tabular}{lll}
\hline & First Mode & Second Mode \\
Experimental Average & 142.8 & 800.88 \\
Theoretical (FEA) w/ Accelerometer & 144.86 & 855.735 \\
Error Percentage (\%) & 1.44 & 6.85 \\
Theoretical (FEA) w/o Accelerometer & 195.132 & 647.29 \\
Error Percentage (\%) & 36.65 & 19.07 \\
\hline
\end{tabular}

\subsubsection{Constructing the Delaminated Finite Element Model Cases}

The steps in creating the delaminated beam models in GeoStar were almost identical to the plate models. The only difference between the two models was the number of elements used on the model. For the beam geometry model, the volumetric mesh consisted of 80 elements for the length and 8 elements for the width of the facesheet, but the thickness consisted of two elements. Two elements were selected in order to have one per lamina of carbon fiber. The volumetric mesh for the foam core consisted of 80 elements for the length and 8 elements for the width of the foam, while the thickness consisted of 10 elements. The accelerometer mass force was applied by replicating the accelerometer onto the beam; four inches from the base of the free beam and directly in the center of the beam. Constraints were applied onto the model and the frequency analysis option was selected to perform modal analysis on the finite element model. Figure 76 shows the results of the FEA model of the first two modal shapes of the middle delaminated composite sandwich beam. From the numerical results, the resonance values are obtained to compare the experimental values. 

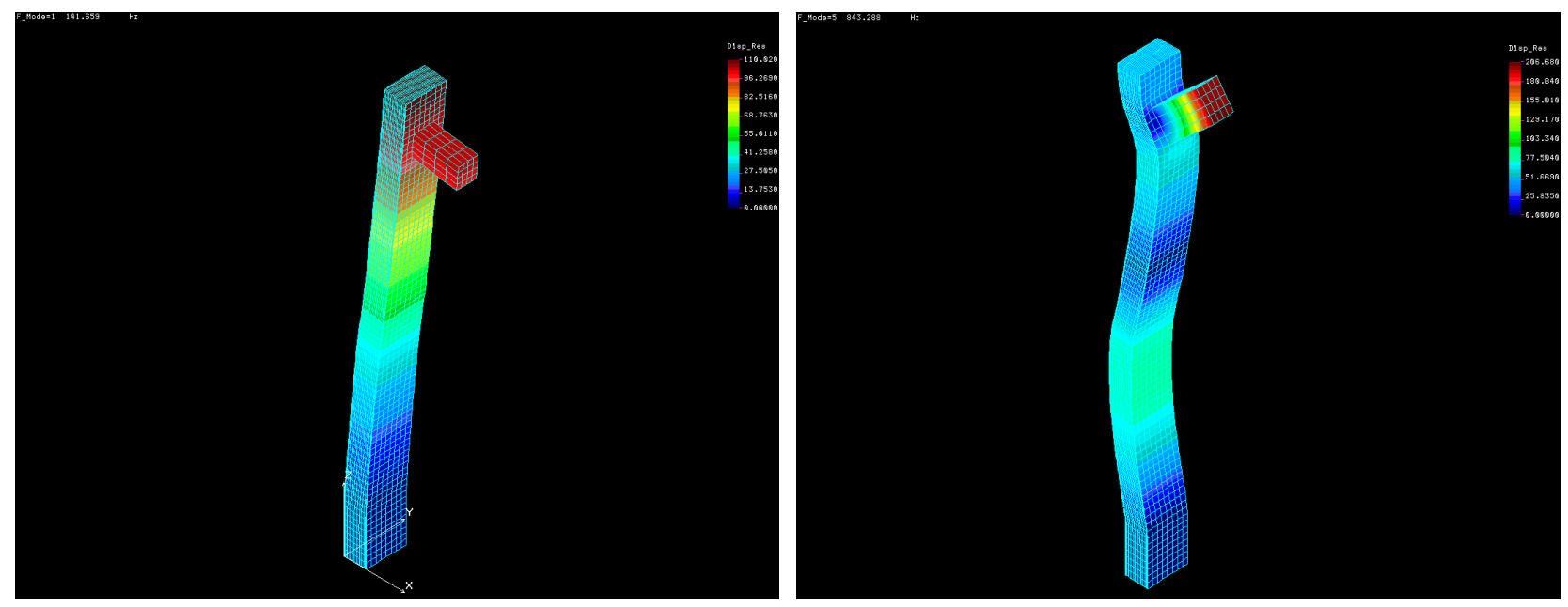

Figure 76: Finite element results for the middle delaminated case study.

For the experimental analysis, three different orientations of the delamination region were vibrated. The same method was applied to each model, but the delamination location was shifted to fit the measurements of the manufactured specimen. Table 16 shows the comparison between all the finite element models that were produced. The only case that might be different when comparing the numerical and experimental results is the second mode for the top delaminated case.

Table 16: Finite element results for the first four modes that were detected by the accelerometer for each delaminated only case study.

\begin{tabular}{lll}
\hline & First Mode $(\mathrm{Hz})$ & Second Mode $(\mathrm{Hz})$ \\
Bottom & 142.56 & 841.77 \\
Middle & 141.659 & 843.29 \\
Top & 143.83 & 677.99 \\
\hline
\end{tabular}

\subsubsection{Constructing the Shear Key Finite Element Model Cases}

The steps in creating the shear key-only beam models in GeoStar were almost identical to the plate models. The only difference between the two models was the amount of elements placed on the model. For the beam geometry model, the volumetric mesh consisted of 80 elements for the length and 8 elements for the width of the facesheet, while the thickness consisted of two elements. Two elements were selected in order to have one per lamina of carbon fiber. The volumetric mesh for the foam core consisted of separate blocks to allow the shear keys to have fully aligned nodes when merging. The mass force of the accelerometer was applied by replicating the accelerometer onto the beam four inches from the base of the free beam and directly in the center of the beam. The same boundary condition constraints 
were applied onto the model. Once the boundary conditions and loads are set, the software's analysis program can commence by selecting the frequency analysis option. Figure 77 shows the results of the FEA model of the first two modal shapes of the bottom composite sandwich beam with only shear keys. From the results the resonance values are obtained to compare the experimental values.
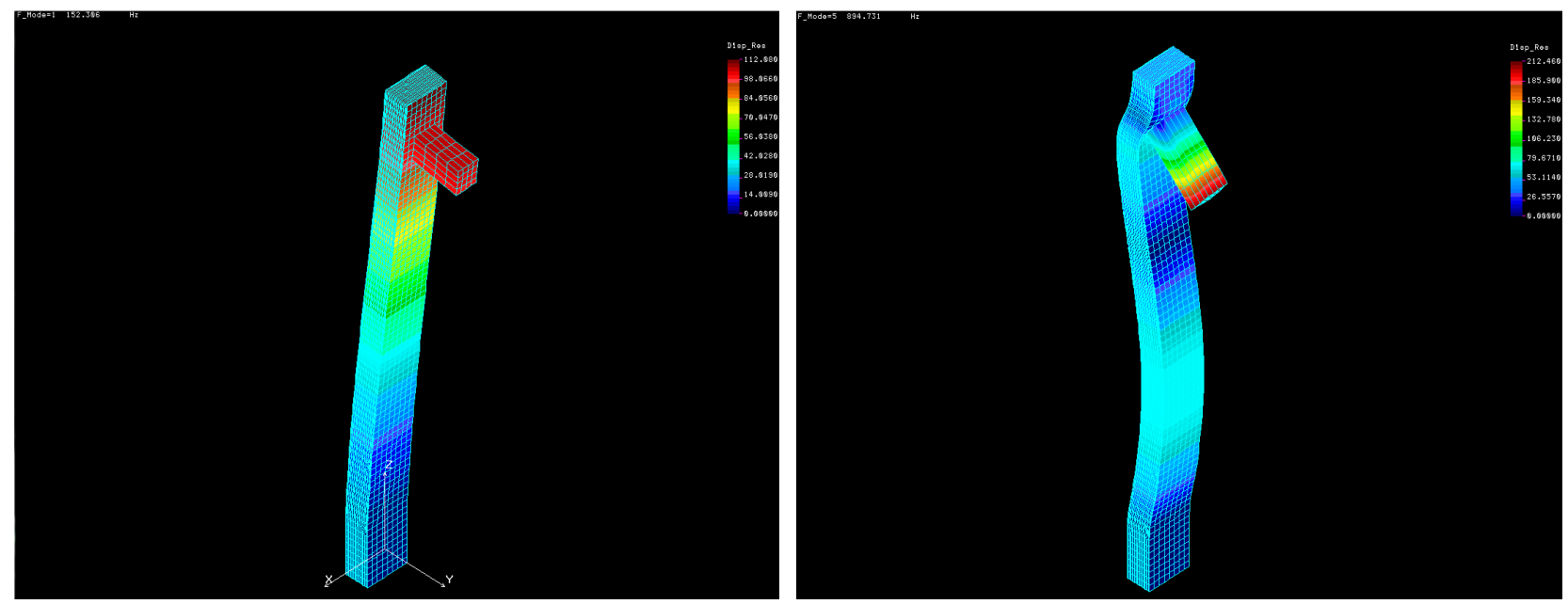

Figure 77: Finite element results for the bottom shear key-only case study.

For the experimental analysis there were three different orientations of the delamination region that were vibrated. The same method was applied to each model, but the delamination location was shifted to fit the measurements of the manufactured specimen. Table 17 shows the comparison between all the finite element models that were produced. Unlike the previous case, where one model didn't have similar values, all the models for the first two modes were similar. The models had similar results with slight differences in the values, which were expected because the experimental results were the identical for each case.

Table 17: Finite element results for the first two bending modes for each delaminated only case study.

\begin{tabular}{lll}
\hline & First Mode $(\mathrm{Hz})$ & Second Mode $(\mathrm{Hz})$ \\
Bottom & 152.31 & 894.73 \\
Middle & 150.76 & 886.64 \\
Top & 145.79 & 954.64 \\
\hline
\end{tabular}




\subsubsection{Constructing the Delaminated Finite Element Model with Shear Key Cases}

Similar to the shear key-only model, the combination of both shear key and delamination had the same geometry and mesh exception for the merging of the nodes. The steps in creating the combination of both shear key and delamination beam models in GeoStar were almost identical to the plate models. The only difference between the two models was the amount of elements used. The accelerometer mass force was applied by replicating the accelerometer onto the beam four inches from the free end and centered. Once the boundary conditions and loads were implemented, the software's analysis program was run by selecting the frequency analysis option. Figure 78 shows the results of the FEA model of the mode shapes of the composite structure for the top case study. The FEA resonance values were compared to the experimental values.
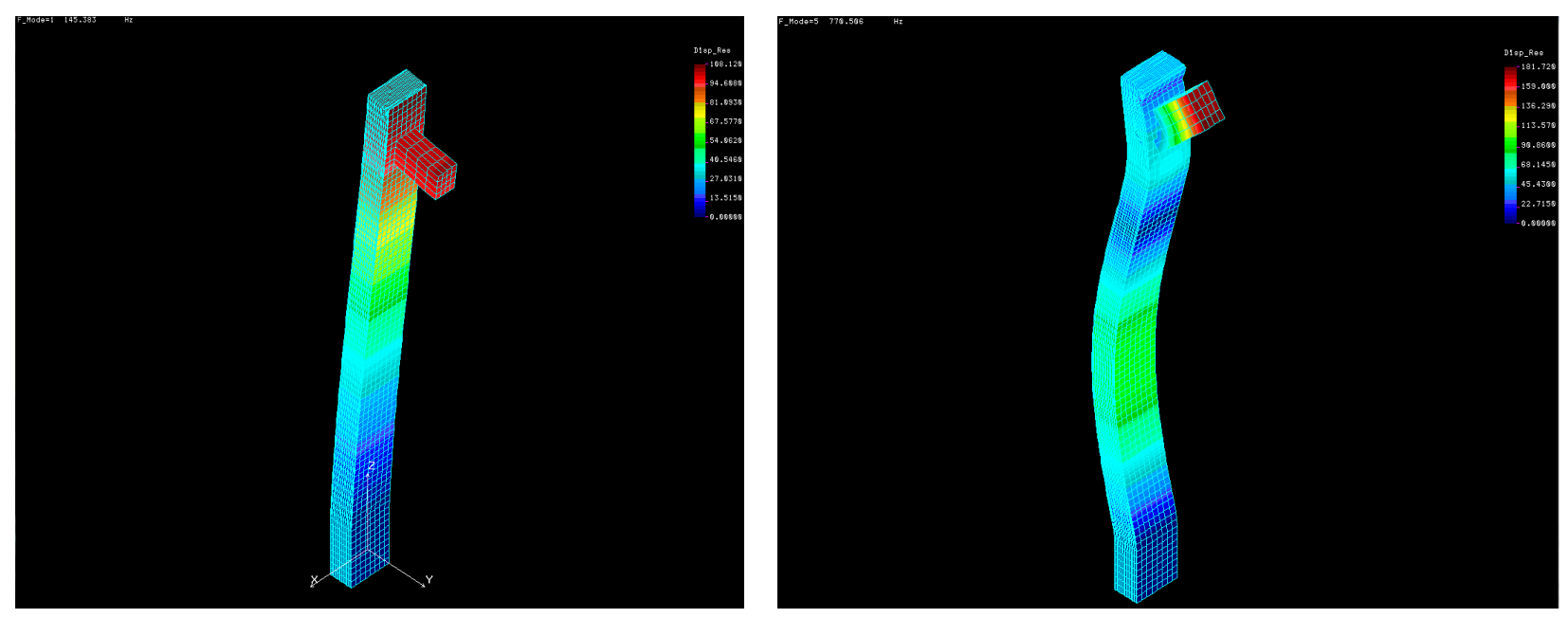

Figure 78: Finite element models of the top delaminated with shear keys case using GeoStar and COSMOS to compare the modes detected by the accelerometer.

Three different orientations of the delamination region were vibrated for the experimental analysis. The same method was applied to each model, but the delamination location was shifted to fit the measurements of the manufactured specimen. Table 18 shows the comparison between all the finite element models produced. All the models for the first two modes were similar. The models had similar results with slight differences because the experimental results varied for each case. 
Table 18: The Finite element results for the first two bending modes for each delamination with shear key case study.

\begin{tabular}{lll}
\hline & First Mode & Second Mode \\
Bottom & 150.19 & 884.19 \\
Middle & 149.26 & 882.37 \\
Top & 145.38 & 770.51 \\
\hline
\end{tabular}

\subsubsection{Constructing the Vertical Shear Key Finite Element Model Cases}

The finite element models that incorporated shear keys placed lengthwise along the beams were created differently than the other beam cases. The base of the beam was created instead of the length of the beam for this iteration. In this way, the geometry of the shear key could be captured and then extruded 10 inches from the base. Creating the shear key followed the previous methods where the model was slit into sections in order to align the nodes when merged. If the model did not have a shear key, then the foam core was a $1 \times .5$ inch block instead of three separate pieces. The carbon fiber facesheets stayed the same with 80 elements on the length of the beam and 8 elements on the width of the beam. Two elements were once again selected in order to have one per lamina of carbon fiber. The mass force of the accelerometer was applied by replicating the accelerometer onto the beam four inches from the base of the free beam and directly in the center. Once again the same boundary condition constraints were applied onto the model. Figure 79 shows the results of the FEA model of the first two bending mode shapes for the 2 inch delaminated composite sandwich beam. The two top illustrations show the results without a shear key embedded into the foam while the other two show the results with a shear key inside the composite. From the results the resonance values were obtained to compare the experimental values.
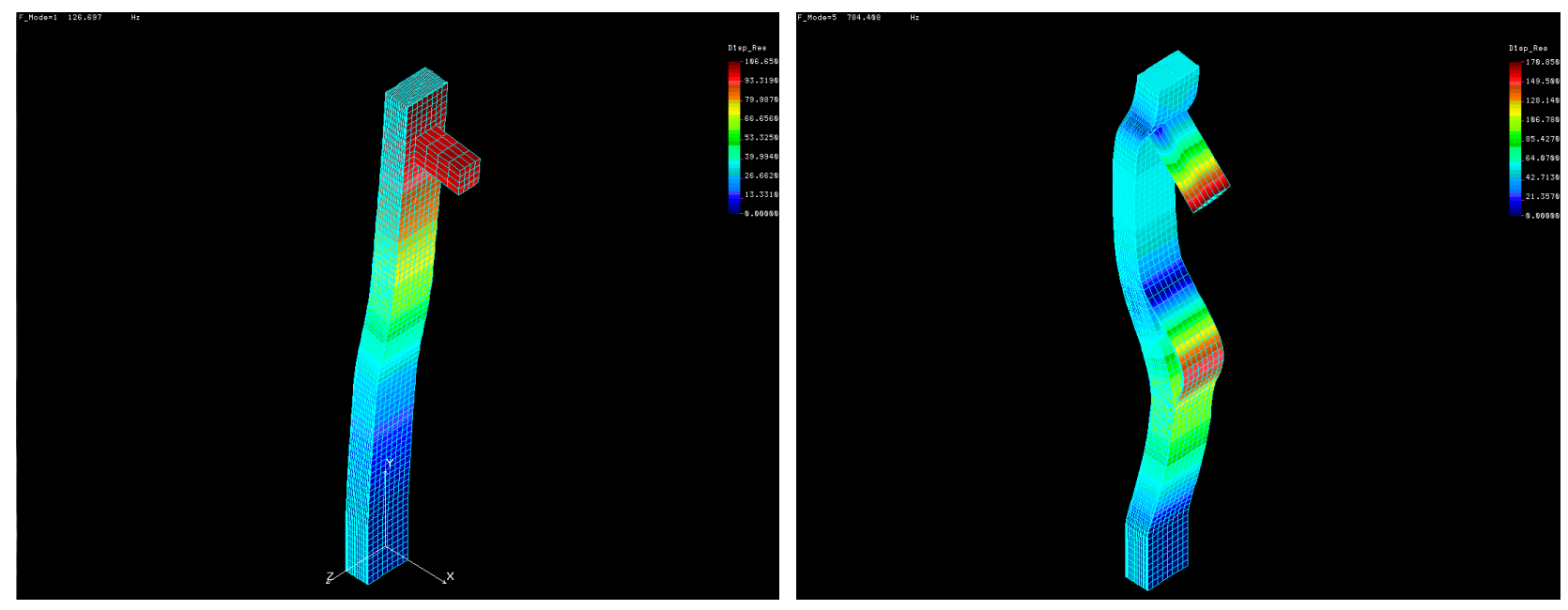

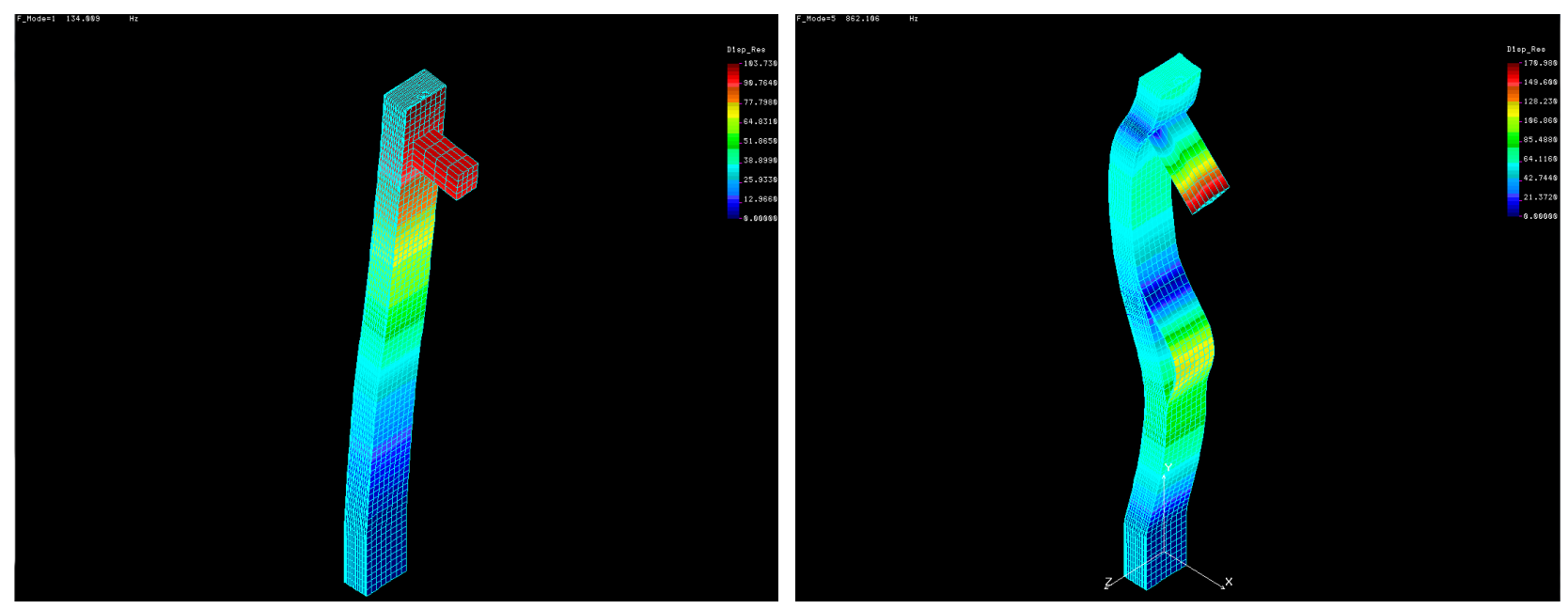

Figure 79: Finite element models of the 2-inch delamination without shear keys in the top row and with shear keys in the bottom row for the first two bending natural frequency.

Seven different case studies were performed for the shear key embedded into the foam along the length. The same method was applied to each model, but the delamination region changed for the cases with and without the shear keys inside the composite beam. Table 19 shows the comparison between all the finite element models produced. Similar to the experimental results, most of the sandwich composites with shear keys had a higher frequency than those without. Nonetheless, these models were compared to the experimental results to validate the data for this research.

Table 19: The Finite element results for the first two bending modes of the vertical shear key case study.

\begin{tabular}{lll}
\hline & First Mode & Second Mode \\
Control & 144.86 & 855.735 \\
Shear Key Control & 142.28 & 951.52 \\
1 inch delame w/o shear key & 138.74 & 824.34 \\
2 inch delame w/o shear key & 126.7 & 784.41 \\
3 inch delame w/o shear key & 105.95 & 755.94 \\
1 inch delame w/ shear key & 137.614 & 893.672 \\
2 inch delame w/ shear key & 134.01 & 862.11 \\
3 inch delame w/ shear key & 127.97 & 834.62 \\
\hline
\end{tabular}




\section{Comparison Between Numerical and Experimental Analysis}

The numerical results were validated by comparing them to the experimental values. Once the model properly represented the experimental results and trends it could be used to investigate the shear keys more thoroughly. The resonance values for both the finite element models and the experimental results were compared graphically and through the comparison of error percentage to determine how the two results deviated from one another.

\subsection{Graphical Representation of the Numerical and Experimental Results}

Data from both the finite element models and the experimental results were placed in Matlab to determine the trends of both types of analyses .To further investigate the trends of the control specimen and finite element analysis, a graphical representation of the resonance values was created, as depicted in Fig. 80. This figure shows the comparison of the finite element resonance in comparison to all three control plates. The four graphs follow a similar trend from the first, second, and fourth modes. However, on the third mode the numerical value increased dramatically when compared to the control specimens. The third mode had a pure bending motion when modeling the composite sandwich on the FEA program. Even though there were other modes on the FEA program that had a closer value to the experimental data, the bending modal shape would not be the mode selected in every case This gave a better insight as to why the finite element program only had accurate values for the first two bending modes. Another interesting observation from the figure was the similarities between the third specimen and the finite element values. 


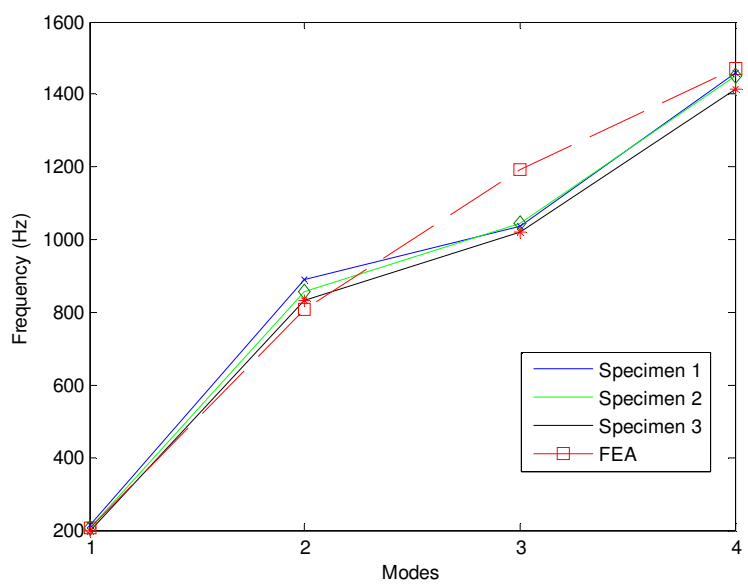

Figure 80: Graphical representation between the experimental and finite element results for the control sandwich composite plate.

To further investigate the trends of the experimental results of the middle case and finite element analysis, a graphical representation of the resonance values is depicted in Fig. 81. This figure shows the comparison of the finite element resonance in comparison to all the sub-cases that include control, delamination only, shear key-only, and a combination of both shear key and delame. The lines of the graph followed a similar trend for the first, second, and fourth modes. However, on the third modes the numerical value increased drastically for all the finite element cases. The rest of the composite sandwich cases showed a similar trend where the third mode was completely different from the rest of the experimental results. One reason for this could have been that the mode model acquired for the third mode was wrong for all the cases. Not knowing whether the accelerometer captured the resonance of a torsion mode or a bending mode was difficult to predict. The accelerometer, for the aerospace composite lab, was designed to detect bending modes based on the sensitivity of the accelerometer, which was not capable of detecting pure torsion. The accelerometer used for this research could only detect unidirectional movement. Therefore, the bending modes were selected first when making the finite element models align with the values from the accelerometer. For most of the plate cases, the first and third models for the finite element programs were bending modes, while the second and fourth were a combination of torsion and bending. It was unlikely that the accelerometer could not detect the second bending mode for all the plates. Therefore, the third mode was chosen to be the bending mode. If the third resonance value was not chosen, then all the modes for all the different cases would have varied greatly. Some modes would have a third mode similar to the second or fourth mode resonance values. Therefore, to have consistent data the modes chosen were for a bending mode. 


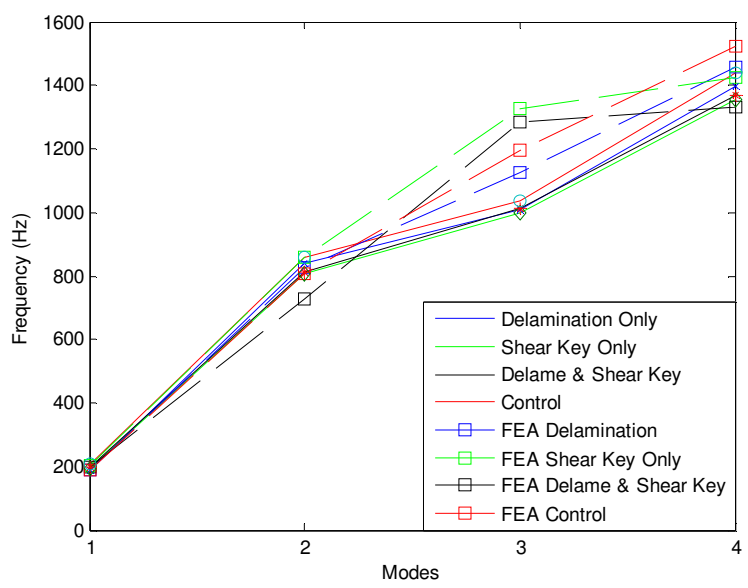

Figure 81: Middle case study comparison between the numerical and experimental results.

When comparing the beam cases, only two finite element models were needed to compare to the experimental values. Unlike the previous plate models, the beam models experienced a bending natural frequency, which was what the accelerometer detected. For this experiment there were three case studies that were modeled in GeoStar. However, the middle case and bottom case studies had similar results when comparing the finite element mode frequencies and the frequencies obtained through the experimental vibration analysis. Figure 82 graphically illustrates the trends between the numerical and experimental results for the middle beam case. For this illustration the finite element modes are relatively close to the values obtained by the vibration table in the first mode. In terms of the second mode, the values from the numerical analysis diverged from the experimental values. However, by comparing the values by the error percentage the difference were not significant.

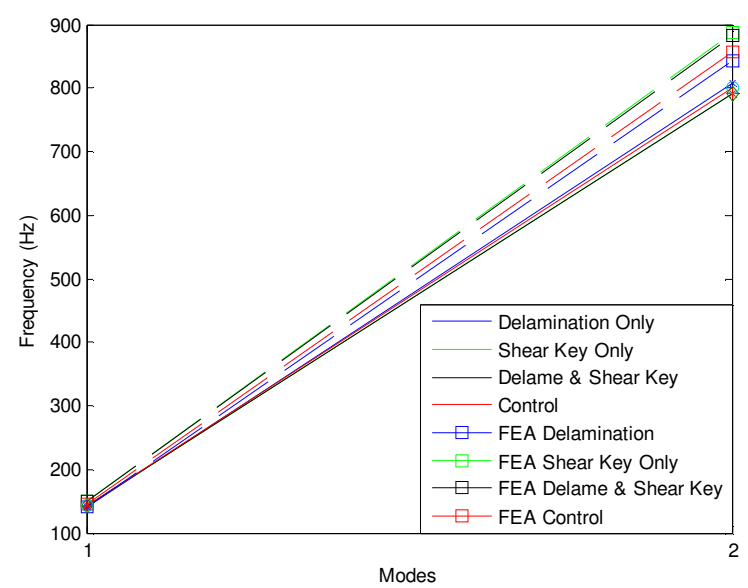

Figure 82: Graphical representation of the finite element and experimental results from the middle beam subcase. 
For the top beam case, there were slight differences when comparing the values of the finite element modes to the values acquired by the vibration analysis. Figure 83 illustrates the trend for the numerical and experimental resonance frequencies for the top beam case. In this graphical representation the numerical values did not behave the same as the experimental values in terms of frequency. In the case of the finite element modes the specimens with shear keys had a higher first natural frequency than the rest of the cases. In the experimental analysis the shear key specimens had the lowest natural frequency values when compared to the rest of the sub-cases. The second mode, the finite element model with shear keys and delamination had a lower natural frequency than the experimental values. For the middle and bottom cases, the ranking order between the numerical and experimental values was similar to each case. However, for the top case the numerical and experimental values did not correspond to one another.

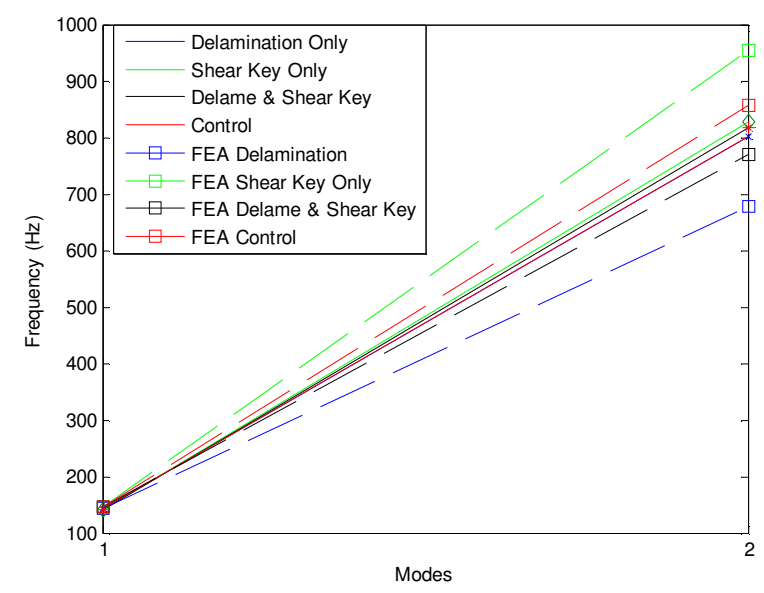

Figure 83: Graphical representation of the finite element and experimental results from the top beam subcase

The last experiment conducted for this research involved determining whether increasing the delamination region with a vertical shear key embedded into the structure would affect the natural frequency of the specimen. In this experiment the finite element modes were closer to the numerical and experimental values than in the composite sandwich beam and plate. Figure 84 compares the finite element values to the values obtained from the vibration table. In the figure, the top graph shows all the average experimental results and all the numerical results for each sub-case. The dashed lines indicate finite element values while the solid lines indicate the average experimental values. The bottom graph shows the trends for the 3 inch delaminated case only. In this graph the control, shear key-only, delamination only, and delamination with shear key are depicted in the figure. By closely examining the 
graph it is noticeable that some of the trends were nearly identical when comparing the experimental and numerical analysis. However, in the 3 inch delaminated case and as well as the other cases there were several trends that did not relate to each other. In this case study the finite element shear key with delame model is lower for both the first and second modes. While in the experimental analysis the shear key with delamination had a higher frequency for both modes. Nonetheless, there were various similarities between the experimental analysis and numerical analysis.
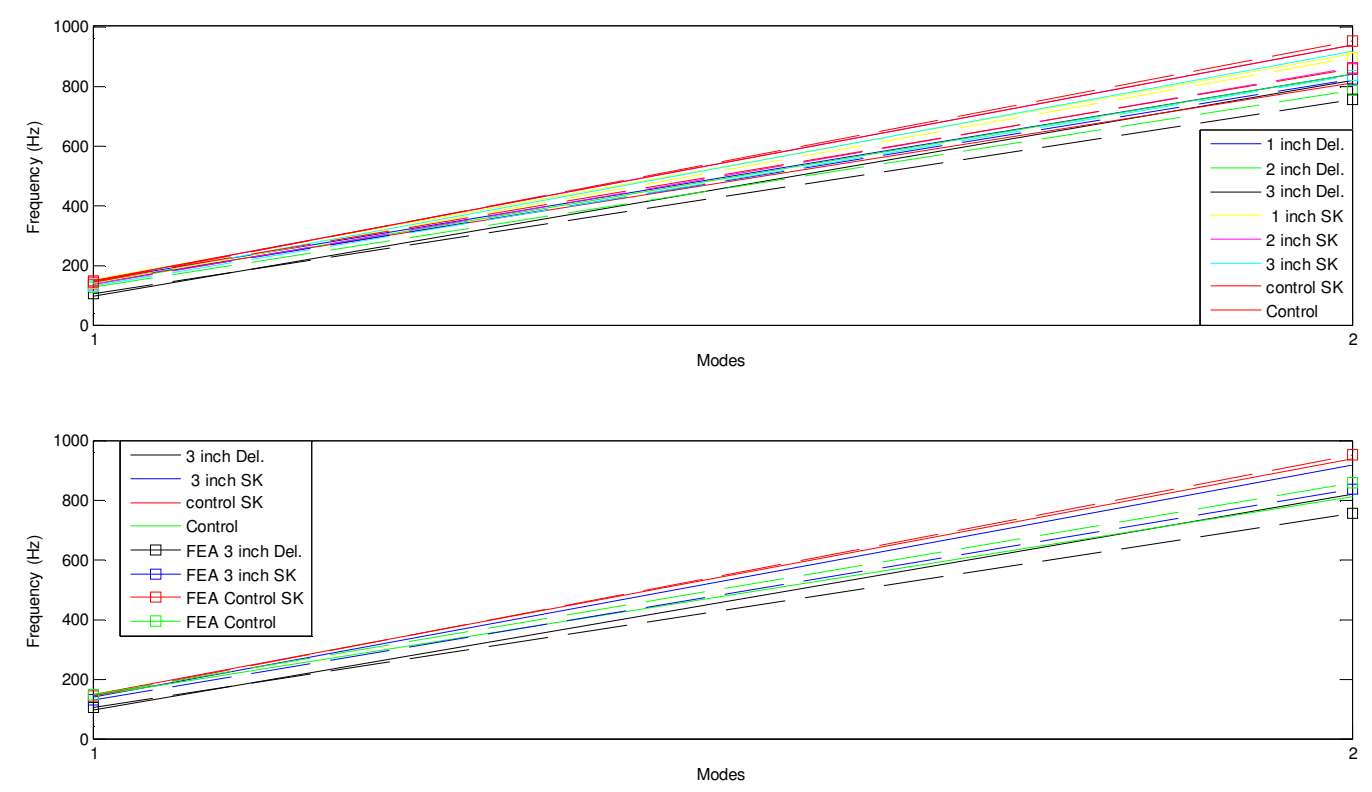

Figure 84: Depiction of the numerical and experimental results for the vertical shear key case for different sub-cases and delamination regions.

\subsection{Error Percentage between the Numerical and Experimental Results}

In order to fully understand whether the experimental values compare to the numerical values, the error percentages between the two analyses was done. In total there were 64 finite element models created in order to analysis all the results obtained from the experimental analysis. Table 20 shows the results for the middle and top cases for all the specimens. There are wide varieties of error percentages for the middle and top cases with values range from $.18 \%$ to $16.88 \%$. The majority of the large error percentage originates from the specimens with shear keys embedded into the structure. These large values could have been caused by certain factors from the manufacture methods or the material properties. 
Table 20: Shows the error percentage among the numerical and experimental results for several case studies.

\begin{tabular}{|c|c|c|c|c|c|c|c|c|c|c|}
\hline \multirow[t]{2}{*}{$\begin{array}{l}\text { Composite } \\
\text { Orientation }\end{array}$} & \multicolumn{2}{|c|}{ Plates } & \multicolumn{2}{|c|}{ Beams } & \multicolumn{2}{|c|}{$\begin{array}{c}\text { 1-inch } \\
\text { Delamination }\end{array}$} & \multicolumn{2}{|c|}{$\begin{array}{c}\text { 2-inch } \\
\text { Delamination }\end{array}$} & \multicolumn{2}{|c|}{$\begin{array}{c}\text { 3-inch } \\
\text { Delamination }\end{array}$} \\
\hline & $1^{\text {st }}$ & $2^{\text {nd }}$ & $1^{\text {st }}$ & $2^{\text {nd }}$ & $1^{\text {st }}$ & $2^{\text {nd }}$ & $1^{\mathrm{st}}$ & $2^{\text {nd }}$ & $1^{\text {st }}$ & $2^{\text {nd }}$ \\
\hline Control & $9.19 \%$ & $6.38 \%$ & $1.44 \%$ & $6.85 \%$ & $1.44 \%$ & $6.85 \%$ & $1.44 \%$ & $6.85 \%$ & $1.44 \%$ & $6.85 \%$ \\
\hline Middle Delame & $1.05 \%$ & $1.39 \%$ & $.18 \%$ & $4.42 \%$ & $1.44 \%$ & $6.85 \%$ & $1.44 \%$ & $6.85 \%$ & $1.44 \%$ & $6.85 \%$ \\
\hline $\begin{array}{l}\text { Middle Shear } \\
\text { Keys Only }\end{array}$ & $3.85 \%$ & $6.14 \%$ & $5.12 \%$ & $12.19 \%$ & $5.74 \%$ & $1.99 \%$ & $7.38 \%$ & $6.48 \%$ & $9.41 \%$ & $7.82 \%$ \\
\hline $\begin{array}{l}\text { Middle Delame } \\
\text { w/ Shear Keys }\end{array}$ & $1.69 \%$ & $10.08 \%$ & $3.65 \%$ & $11.61 \%$ & 7.64 & $1.18 \%$ & $2.68 \%$ & $1.57 \%$ & $2.68 \%$ & $1.57 \%$ \\
\hline Top Delame & $2.18 \%$ & $1.39 \%$ & $.36 \%$ & $4.42 \%$ & $\mathrm{NaN}$ & $\mathrm{NaN}$ & $\mathrm{NaN}$ & $\mathrm{NaN}$ & $\mathrm{NaN}$ & $\mathrm{NaN}$ \\
\hline $\begin{array}{l}\text { Top Shear } \\
\text { Keys }\end{array}$ & $.67 \%$ & $2.14 \%$ & $2.65 \%$ & $15.23 \%$ & $\mathrm{NaN}$ & $\mathrm{NaN}$ & $\mathrm{NaN}$ & $\mathrm{NaN}$ & $\mathrm{NaN}$ & $\mathrm{NaN}$ \\
\hline $\begin{array}{l}\text { Top Delame w/ } \\
\text { Shear Keys }\end{array}$ & $10.05 \%$ & $16.88 \%$ & $3.47 \%$ & $5.58 \%$ & $\mathrm{NaN}$ & $\mathrm{NaN}$ & $\mathrm{NaN}$ & $\mathrm{NaN}$ & $\mathrm{NaN}$ & $\mathrm{NaN}$ \\
\hline
\end{tabular}

While manufacturing the composite sandwich specimens with shear keys embedded into the structure, the shear keys could have not fully connected to the foam while curing. Even though a thin layer of aeropoxy resin was place between the foam and shear key, the high temperature in the autoclave could have cause the resin to evaporate while curing. Another explanation for this data could have been that the material properties of the fiberglass strands were not the properties of the shear keys. When conducting the material property tests of the fiberglass shear keys, strands of fiberglass were laid flat in a unidirectional orientation and aeropoxy resin was applied onto the fibers. However, the geometry of the shear keys was a semi circle, which might have altered the material properties. The shear keys could not have been placed inside the Instron machine to determine the material properties because the clamps on the Instron machine would have fractured the specimen. Therefore, laying up a flat plate with unidirectional fiberglass was the best option.

The rest of the results from the error percentage showed a smaller range of values. Further analysis of all the specimens shows that the total percentage of the specimens with an error percentage of over $10 \%$ is only $12.25 \%$ of all the models. This percentage is decreased to $4.68 \%$ when examining the results that had an error percentage of over $15 \%$. Therefore, when determining the error percentages of all 64 models, the results are conclusive that the numerical and experimental values are equivalent. 


\section{Conclusion}

Previous studies on shear keys embedded into a foam sandwich core have shown improved overall material strength in terms of bending and shear force. However, from this research it is evident that shear keys inside a beam sandwich composite were unsuccessful in terms of improving the vibration response of the system. While having shear keys embedded into a sandwich beam increases the first and second natural frequency values, the total weight and amplitude values were also increased at the resonance frequencies. When comparing the resonance frequency to weight ratio at the first mode for specimens with shear keys and specimens without shear keys; it becomes evident that shear keys are not beneficial. The only case where shear keys would help the vibration response without affecting the weight of the structure would be when the delamination region has expanded over $25 \%$ of the surface between the facesheet and core.

The results from the composite sandwich plate indicated a different result from the beam composites. The composite plates had a slight increase in the resonance frequency when comparing delaminated specimens with and without shear keys. However, the resonance frequency to weight ratio of the specimens was more promising when comparing to the delaminated-only cases. The weight of the two shear keys embedded into the foam did not dramatically increase the overall weight of the structure like the beam cases. Therefore, the resonance frequency to weight ratio was larger for the delaminated with shear key specimens than specimens without shear keys. The amplitude of the shear key specimens also had a lower value when compared to specimens without shear keys. The results in the time domain indicated specimens with shear keys would dampen out more slowly than specimens without shear keys. Using the dampening ratio for each case showed that specimens with shear keys would harm the response of the system. The results of this research concluded that applying shear keys into a sandwich composite plate would assist only in frequency response of the structure.

Orientation of the delamination had some effect when analyzing the frequency response of the composite structure. In some cases the delamination orientation would behave similar to other orientation locations. An example of this was for the bottom and top cases of the beam structure, where both the amplitude and frequencies at each mode behaved the same. The middle beam cases had a familiar frequency trend, but the amplitude graph showed that the delaminated specimen with shear keys had the highest amplitude for the first and second modes, where in the other two cases it was the specimen with only shear keys inside the structure. Along with similarities for the different cases, there were also some differences among the specimens. One case that was completely different from the rest was when the delamination and shear keys were applied vertically onto the plate's side. The amplitude for the second mode had the highest value for all the different sub-cases. The other orientations concluded that the first 
mode experienced the highest amplitude instead of the second mode. These results would suggest that the orientation of the shear keys and delamination might affect the frequency response of the structure. However, there is strong evidence suggesting that certain orientations will also perform the same as other orientations of the delamination and shear key placement.

Validation of the experimental results was done by comparing the values from the finite element analysis to the value acquired from the experimental vibration analysis. The error percentage between the two values was taken in order to determine whether the experimental values and numerical values were comparable. The results of this comparison showed that $83.07 \%$ of the data had an error percentage less than $10 \%$. The rest of the results with a higher error percentage could have originated from the fact that the fiberglass material properties were not entirely the same values from the shear keys. This error between the material properties could have altered the results from the finite element models and essentially cause the difference between the numerical and experimental results. Nonetheless, a majority of the experimental specimens, analytical results, and the finite element models are related to one another, thus validating the results.

The data collected from these results, can also be used to further analyze and compared to a dynamic application. For this iteration, the composite sandwich structures were vibrated using forced sinusoid motion. If results were collected from a real application scenario, like a rocket fin or aircraft wing experiencing flutter effects, the two results can be compared to determine whether the results from this research accurately represent the real life model. These results can also be used for a health monitoring system to determine if there is a delamination within a composite structure. Further analysis can be implemented to acquire a vibration spectrum to when a composite sandwich specimen is delaminated. Nonetheless, even thought for this research there wasn't a substantial amount of evidence suggesting that shear keys are beneficial to dynamic application, there are still other forms of dynamic motion that can still be studied to determine if there are benefits of using shear keys.

\subsection{Lessons Learned Throughout the Research}

There are two main lessons learned from conducting this research that should be addressed in the future. If comparisons between finite element models and experimental models are required, conducting both experiments simultaneously is recommended. In some cases, the data acquired from the experimental results could not resemble the results from the finite element models. In this research the foam material properties were not accurately acquired; therefore, rendering the finite element models 
inaccurate. The mistake was caught by performing the analysis methods simultaneously. However, there was a mistake with the fiberglass properties, which was not caught until the end. Repeating the procedures for acquiring the material properties for the fiberglass strands would improve the overall error percentage in the experimental and numerical data. However, over 95\% of the FEA models had less than $15 \%$ error between the numerical and theoretical results. If the shear key properties were re-evaluated, it would have not significantly increased the validation of the experimental and numerical results.

The last lesson learned from this research was that the type of element shape and formulation is important for the finite element analysis. Depending on the element shape and type could easily change the finite element results. However, for this research the sensitivity analysis of the different element shapes was not considered; even thought it would have increase the fidelity of this research.

\subsection{Future Work}

There are two experiments that resonate when discussing shear keys regarding the vibration results. During the timeline of this thesis the impact machine located inside the aerospace composites lab was not operating. However, for future research this machine will be running with correct specifications for testing. The impact machine is used to delaminate a sandwich composite instead of placing a delamination during the lay-up and curing cycle. Specimens could be manufactured without a delamination and implemented onto the machine. After the specimen has been delaminated, the composite plate or beam can be vibrated in order to compare results from this thesis. However, if the researcher wanted to test another vibration aspect, then the specimen could be vibrated horizontally instead of vertically.

By placing the specimen inside the horizontal position of the vibration table, the specimen would react differently than in the vertical position. The weight of the specimen would have a different orientation when vibrating, due to the fact that the specimen has a larger gravity load on the body. This larger gravity load would have altered the natural frequency when comparing results from the vertical position of the vibration table. Further fixtures would not be necessary to machine, because fixtures for both the vertical and horizontal positions were made during this research. Nonetheless, there are countless ideas and experiments that can be conducted for composite manufacturing and finite element analysis. I anticipate that future work, along with my research will benefit future researchers when investigating sandwich composites. 


\section{References}

${ }^{1}$ Cooperative Research Centre for Advanced Composite Structures Ltd., Putting it together the science and technology of composite materials. , Australian Academy of Science., November 2000

${ }^{2}$ Doyle, E.J., Suggested Strategies in Screening for Health Effects in Personnel who Work with Composites. Applied Industrial Hygiene Special Issue: December 1989. Pp 64-67.

${ }^{3}$ Jones, R.M., Mechanics of Composite Materials, McGraw Hill, 1975. Print.

${ }^{4}$ Zenkert, D., The Handbook of Sandwich Construction, 1st ed., Engineering Materials Advisory Services, United Kingdom, 1997, Chaps. Intro, Testing.

${ }^{5}$ ASTM Standard D1621, —Compressive Properties of Rigid Cellular Plastics. $\square$ ASTM International. West Conshohocken, PA., 2010.

${ }^{6}$ Megson, T. H.G., Aircraft Structures for Engineering Students, John Wiley \& Sons, New York, 1990.

${ }^{7}$ Jakobsen, J., Bozhevolnaya, E., and Thomsen, O. T., New Peel Stopper Concept for Sandwich Structures,. Aalborg University, Denmark., Science Direct 3378-3385, April. 2007.

${ }^{8}$ Grenestedt, J., Development of a New Peel-Stopper for Sandwich Structures,. Lehigh University, P.A., Science Direct 1555-1559, April. 2001.

${ }^{9}$ Wisnom, M. R., and Jones, M. I., Delamination Due to Interaction Between Overall Interlaminar Shear and Stresses at Terminating Plies,. University of Bristol, United Kingdom., Science Direct 39-47, 1995.

${ }^{10}$ Burman, M., and Zenkert, D., Fatigue of Foam Core Sandwich Beams, Part II: Effect of Initial Damages", International Journal of Fatigue, Vol, 19, Nov. 1997.

${ }^{11}$ Kim, J. H., Lee, Y. S., Park, B. J., and Kim, D. H., Evaluation of Durability and Strength of Stitched Foam-Cored Sandwich Structures,. Chungnam National University, Taejon, South Korea., Elsevier Science, Aug. 2000. 
${ }^{12}$ Mitra, Nilanjan and Kasper, Eric,. " A Novel Technique for Preventing Delamination of the Composite Skin from the Core: Sandwich Panelswith Shear Keys",. California Polytechnic State University, San Luis Obispo : California Central Cost Research Partnership, 2005

${ }^{13}$ Balabat, Richard,. "In Plane Compression Response of Composite Sandwich Plate with Center Hole”,. San Luis Obispo,. California Polytechnic State University, 2009.

${ }^{14}$ Surano, Dominic,. "The Effectiveness of Damage Arrestment Devices in Delaying Fasten-hole Interaction Failure in Carbon Fiber Polyurethane Foam Composites Sandwich Panel Subjected to Static and Dynamic Loading Under Increased Temperatures"., San Luis Obipso., California Polytechnic State University, 2012.

${ }^{15}$ Tran, Tony,. "An Investigation of Initially Delaminated Composite Sandwich with Delamination Arrest Mechanism under Buckling Loading”,. San Luis Obispo,. California Polytechnic State University, 2009.

${ }^{16}$ Woo, Tomas., "Effects of Seawater on the Mechanical Behavior of Sandwich Composite Panels under Monotonic Shear Loading"., San Luis Obipso., California Polytechnic State University, 2012.

${ }^{17}$ Tsai, S. W., Gay, D., and Hoa, S. V., Composite Materials: Design and Application, 4th ed., CRC Press LLC., Boca Raton, FL, 2003.

${ }^{18}$ Vhopra, K. Anil., Dynamic of Structure Theory and Application to Earthquake Engineering, Simon \& Schuster Company., Upper Saddle River, New Jersey 1995

${ }^{19}$ ASTM Standard D3039, - Tensile Properties of Polymer MatrixComposite Materials. $\square$ ASTM International. West Conshohocken, PA., 2008.

${ }^{20}$ ASTM Standard E132, - Poisson's Ratio at Room Temperature. $\square$ ASTM Inernational. West Conshohocken, PA., 2010.

${ }^{21}$ Advanced Composites Group, —LTM 45-1 Component Prepreg $\square$. Umeco Composites. Debrishire, UK. 2009.

${ }^{22}$ ASTM Standard D1621, - Compressive Properties of Rigid Cellular Plastics. $\square$ ASTM International. West Conshohocken, PA., 2010.

${ }^{23}$ General Plastics Company, —Last-A-Foam 6700 Series Property Graphs $\square$. General Plastics Manufacturing Company. Tacoma, WA. 2008. 
${ }^{24}$ Structural Research and Analysis Corporation (Multiple Authors), COSMOS/M Basic FEA System, 2nd ed., Structural Research and Analysis Corporation, United States, October 1998, Chap. 5.

${ }^{25}$ J. N. Reddy., Mechanics of Laminated Composite Plates and Shells: Theory and Analysis., 2nd ed. London., CRC Press(2003) 
fnsfea_skd=[ $\left[\begin{array}{llll}197.417 & 836.36 & 1235.02\end{array}\right.$ 1370.9];

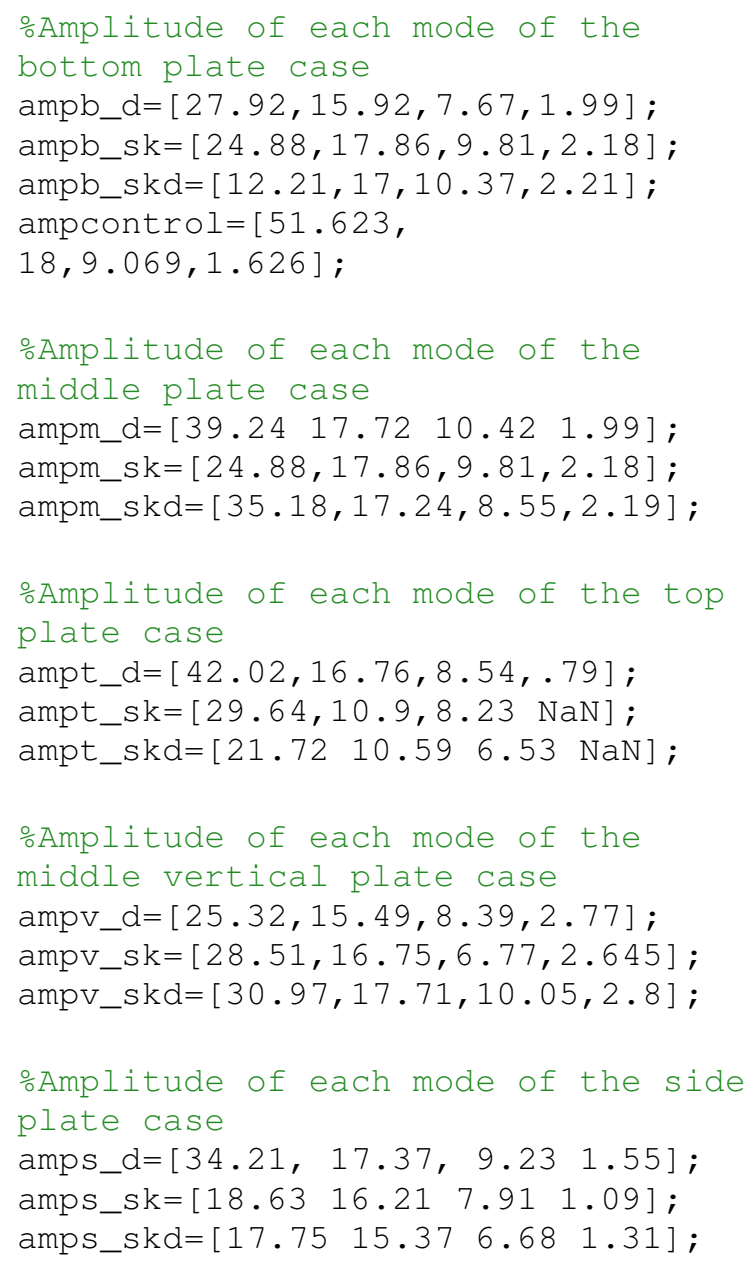

\section{$\because \mathrm{Plots}$}

\section{figure (1)}

plot (modes, fnb_d, 'b', modes, fnb_sk, ' $g^{\prime}$, modes, fnb_skd, 'k', modes, fn_contr ol, 'r')

hold on

plot (modes, fnbfea_d, '--

bs ', modes, fnbfea_sk, '--

gs ' , modes, fnbfea_skd, '--

ks', modes, fnfea_control, '--rs' )

hold on

plot (modes, fnb_d, 'x', modes, fnb_sk, '

$d^{\prime}$ ', modes, fnb_skd, '*', modes, fn_contr ol, '०')

otitle('Natural Frequencies of the bottom plate case')

xlabel ('Modes')

ylabel ('Frequency (Hz)')

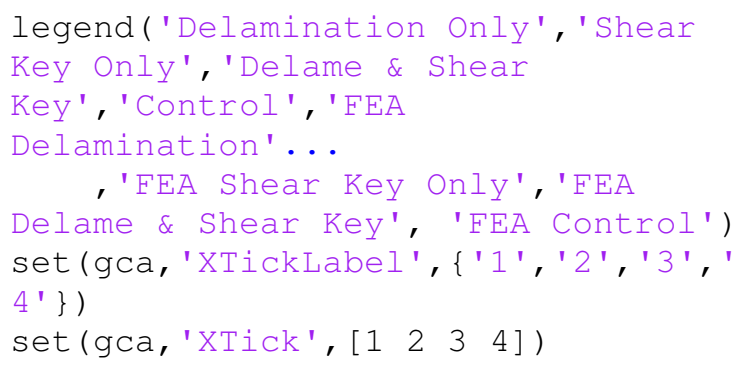


legend('Delamination Only', 'Shear Key Only', 'Delame \& Shear

Key', 'Control')

set (gca, 'XTickLabel', \{'1', '2', '3', ' 4 ' $)$

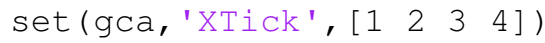

\section{figure (4)}

plot (modes, fnv_d, ' b ', modes, fnv_sk, ' $g^{\prime}$, modes, fnv_skd, 'k', modes, fn_contr ol, 'r')

hold on

plot (modes, fnvfea_d, 'c', modes, fnvfe a_sk, 'm', modes, fnvfea_skd, 'y' )

hold on

plot (modes, fnv_d, 'x', modes, fnv_sk, ' d', modes, fnv_skd, '*', modes, fn_contr ol, 'o')

hold on

plot (modes, fnvfea_d, 'x', modes, fnvfe a_sk, 'd', modes, fnvfea_skd, '*' )

otitle('Natural Frequencies of the

middle vertical plate case')

xlabel ('Modes')

ylabel ('Frequency (Hz)')

legend('Delamination Only', 'Shear

Key Only', 'Delame \& Shear

Key', 'Control', 'FEA

Delamination'...

$$
\text { ,' 'FEA Shear Key Only', 'FEA }
$$

Delame \& Shear Key', 'FEA Control') set (gca, 'XTickLabel', \{'1', '2', '3', ' 4 ' \})

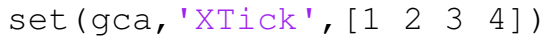

\section{figure (5)}

plot (modes, fns_d, 'b', modes, fns_sk, ' g' $^{\prime}$,modes, fns_skd, 'k', modes, fn_contr ol, 'r')

hold on

plot (modes, fnsfea_d, 'c', modes, fnsfe a_sk, 'm', modes, fnsfea_skd, 'y' )

hold on

plot (modes, fns_d, 'x', modes, fns_sk, ' $d^{\prime}$, modes, fns_skd, '*', modes, fn_contr ol, 'o')

hold on

plot (modes, fnsfea_d, 'x', modes, fnsfe a_sk, 'd', modes, fnsfea_skd, '* ')

otitle('Natural Frequencies of the side plate case')

xlabel ('Modes')

ylabel ('Frequency ( $\mathrm{Hz}$ ) ')

legend('Delamination Only', 'Shear

Key Only', 'Delame \& Shear

Key', 'Control', 'FEA

Delamination'...

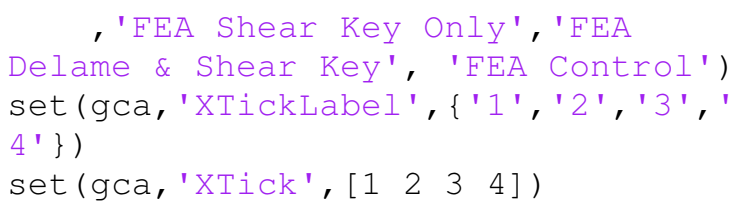

figure (6)

plot (modes, ampb_d, ' b ' , modes, ampb_sk , ' g' ', modes, ampb_skd, 'k ', modes, ampco ntrol, 'r')

hold on

plot (modes, ampb_d, 'x' ', modes, ampb_sk , 'd' ', modes, amplo_skd, ' * ', modes, ampco ntrol, 'o')

otitle('Amplitude of the modes for the bottom plate case')

xlabel ('Modes')

ylabel ('Amplitude (g) ')

legend('Delamination Only', 'Shear

Key Only', 'Delame \& Shear

Key', 'Control')

set (gca, 'XTickLabel', \{'1', '2', '3', ' 4 ' \})

set (gca,' 'XTick', [ [ llll 123 )

figure (7)

plot (modes, ampm_d, ' b ' , modes, ampm_sk ' ' g' ', modes, ampm_skd, 'k ', modes, ampco ntrol, 'r')

hold on

plot (modes, ampm_d, 'x', modes, ampm_sk

, 'd ', modes, ampm_skd, '* ', modes, ampco ntrol, '०')

otitle('Amplitude of the modes for the middle plate case')

xlabel ( 'Modes')

ylabel ('Amplitude (g) ')

legend('Delamination Only', 'Shear

Key Only', 'Delame \& Shear

Key', 'Control')

set (gca, 'XTickLabel', \{'1', '2', '3', ' 4 ' \})

set (gca,' 'XTick', [ [ llll 123 )

figure (8)

plot (modes, ampt_d, ' b' ', modes, ampt_sk ' ' g' ', modes, ampt_skd, 'k' ', modes, ampco ntrol, 'r')

hold on

plot (modes, ampt_d, 'x', modes, ampt_sk , 'd ', modes, ampt_skd, '* ', modes, ampco ntrol, 'o')

otitle('Amplitude of the modes for the top plate case')

xlabel ('Modes')

ylabel ('Amplitude (g) ') 
legend('Delamination Only', 'Shear

Key Only', 'Delame \& Shear

Key', 'Control')

set (gca, 'XTickLabel', \{'1', '2', '3', ' $\left.\left.4^{\prime}\right\}\right)$

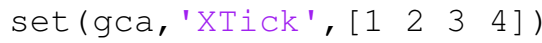

figure (9)

plot (modes, amps_d, 'b' , modes, amps_sk ' ' g' ', modes, amps_skd, 'k' , modes, ampco ntrol, 'r')

hold on

plot (modes, amps_d, 'x', modes, amps_sk ,' 'd' ',modes, amps_skd, '* ' , modes, ampco ntrol, 'o')

otitle('Amplitude of the modes for the side plate case')

xlabel ('Modes')

ylabel ('Amplitude (g) ')

legend('Delamination Only', 'Shear

Key Only', 'Delame \& Shear

Key', 'Control')

set (gca, 'XTickLabel' , \{'1', '2', '3', ' 4 ' \})

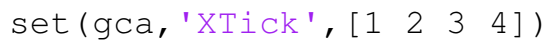

figure (10)

plot (modes, ampv_d, 'b' , modes, ampv_sk , 'g ', modes, ampv_skd, 'k ', modes, ampco ntrol, 'r')

hold on

plot (modes, ampv_d, 'x', modes, ampv_sk , 'd ', modes, ampv_skd, ' * ', modes, ampco ntrol, 'o')

otitle('Amplitude of the modes for

the vertical plate case')

xlabel ('Modes')

ylabel ('Amplitude (g)')

legend('Delamination Only', 'Shear

Key Only', 'Delame \& Shear

Key', 'Control')

set (gca, 'XTickLabel', \{'1', '2', '3', ' $\left.\left.4^{\prime}\right\}\right)$

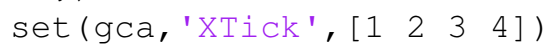

응 Time Domain

Control

fn_control $=[206.57,860.33,1034,1440$

$.67]$;

ampcontrol $=[51.623$,

$18,9.069,1.626]$;

zeta_c $=(1 /(2 * \mathrm{pi})) * \log (51 / 18)$;

$\mathrm{t}=0: \overline{1} / 20 / 1500: .35$;

$\mathrm{s} 1=206.57 *(-$ zeta_c $+i * \operatorname{sqrt}(1-$

zeta_c^2));

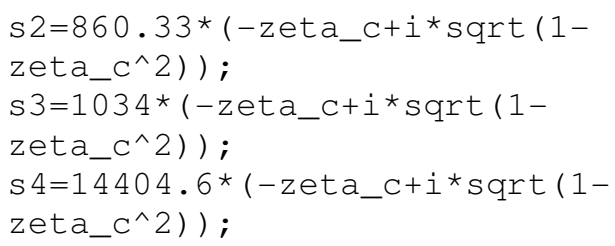


pb_skd $(1,3) * \exp ($ s $3 \mathrm{skd}$ *t $(j))+$ ampb_sk $d(1,4) * \exp \left(s 4 s k d^{*} t(j)\right)$;

end

\section{○Middle}

zeta_md $=(1 /(4 * p i)) * \log \left(\operatorname{ampm\_ d}(1,1) /\right.$ ampm_d $(1,3))$;

sld=fnm_d $(1,1)$ * $(-$ zeta_md $+i * \operatorname{sqrt}(1-$ zeta_md^2));

s2d=fnm_d $(1,2) *\left(-z e t a \_m d+i * \operatorname{sqrt}(1-\right.$ zeta_md^2));

s3d=fnm_d $(1,3) *(-$ zeta_md $+i *$ sqrt $(1-$ zeta_md^2));

s $4 d=f n m \_d(1,4) *\left(-z e t a \_m d+i * \operatorname{sqrt}(1-\right.$ zeta_md^2));

zeta_msk=(1/(4*pi) $) * \log ($ ampm_sk $(1,1$ ) / ampm_sk $(1,3))$;

$\mathrm{s} 1 \mathrm{sk}=\mathrm{fnm} \_\mathrm{sk}(1,1) *(-$

zeta_msk+i*sqrt $(1-$ zeta_msk^2));

s2sk=fnm_sk $(1,2) *(-$

zeta_msk+i*sqrt $(1-$ zeta_msk^2));

s3sk=fnm_sk $(1,3) *(-$

zeta_msk+i*sqrt $(1-$ zeta_msk^2));

$\mathrm{s} 4 \mathrm{sk}=\mathrm{fnm} \_\mathrm{sk}(1,4) *(-$

zeta_msk+i*sqrt $(1-$ zeta_msk^2) );

zeta_mskd=(1/(4*pi ) )*log (ampm_skd (1 , 1 ) / ampm_skd $(1,3))$; slskd=fnm_skd $(1,1) *(-$

zeta_mskd+i*sqrt $(1-$ zeta_mskd^2)); s2skd=fnm_skd $(1,2) *(-$

zeta_mskd+i*sqrt $(1-$ zeta_mskd^2)) ;

s3skd=fnm_skd $(1,3) *(-$

zeta_mskd+i*sqrt $(1-$ zeta_mskd^2) ) ;

s 4 skd $=$ fnm_skd $(1,4) *(-$

zeta_mskd+i*sqrt $(1-$ zeta_mskd^2)) ;

for $j=1:$ length $(t)$

y2_m $(j)=\operatorname{ampm\_ d}(1,1) * \exp (\mathrm{s} 1 \mathrm{~d} * t(j))+\mathrm{a}$ mpm_d $(1,2) * \exp (s 2 d * t(j))+a m p m \_d(1,3$ )$* \exp (\mathrm{s} 3 \mathrm{~d} * t(j))+$ ampm_d $(1,4) * \exp (\mathrm{s} 4 \mathrm{~d}$ $\star t(j))$;

y3_m $(j)=\operatorname{ampm\_ sk}(1,1) * \exp (\operatorname{sisk} * t(j))$ + ampm_sk $(1,2) * \exp (\mathrm{s} 2 \mathrm{sk} * \mathrm{t}(j))+$ ampm_s $\mathrm{k}(1,3){ }^{\star} \exp (\mathrm{s} 3 \mathrm{sk} \star t(j))+$ ampm_sk $(1,4)$ * $\exp (s 4 s k * t(j))$;

y4_m $(j)=$ ampm_skd $(1,1) * \exp (\operatorname{siskd*t~}(j$ )) +ampm_skd $(1,2) * \exp (\operatorname{s} 2 \mathrm{skd} \star t(j))+a m$ pm_skd $(1,3) * \exp (s 3 s k d * t(j))+a m p m \_s k$ $d(1,4) * \exp (s 4 s k d * t(j))$; end

\section{$\because \mathrm{Top}$}

zeta_td $=(1 /(4 * p i)) * \log \left(\operatorname{ampt} \_d(1,1) /\right.$ ampt_d $(1,3))$;

sld=fnt_d $(1,1) *\left(-z e t a \_t d+i * \operatorname{sqrt}(1-\right.$ zeta_td^2));

s $2 d=f n t \_d(1,2) *\left(-z e t a \_t d+i * \operatorname{sqrt}(1-\right.$ zeta_td^2));

s3d=fnt_d $(1,3) *\left(-z e t a \_t d+i * \operatorname{sqrt}(1-\right.$ zeta_td^2));

zeta_tsk $=(1 /(4 * \mathrm{pi})) * \log \left(\operatorname{ampt} \_s k(1,1\right.$ ) / ampt_sk $(1,3))$;

sisk=fnt_sk $(1,1) *(-$

zeta_tsk+i*sqrt $(1-$ zeta_tsk^2) );

$\mathrm{s} 2 \mathrm{sk}=\mathrm{fnt}$ _sk $(1,2) *(-$

zeta_tsk+i*sqrt $(1-$ zeta_tsk^2));

$\mathrm{s} 3 \mathrm{sk}=\mathrm{fnt}$ _sk $(1,3) *(-$

zeta_tsk+i*sqrt $(1-$ zeta_tsk^2));

zeta_tskd=(1/(4*pi) )*log (ampt_skd (1 , 1 ) / ampt_skd $(1,3))$;

slskd=fnt_skd $(1,1) *(-$

zeta_tskd+i*sqrt $(1-$ zeta_tskd^2) );

s2skd=fnt_skd $(1,2) *(-$

zeta_tskd+i*sqrt $(1-$ zeta_tskd^2));

s3skd=fnt_skd $(1,3) *(-$ zeta_tskd-

$i{ }^{\star} \operatorname{sqrt}(1-$ zeta_tskd^2));

for $j=1:$ length $(t)$

y2_t $(j)=a m p t \_d(1,1) * \exp (s 1 d * t(j))+a$ mpt_d $(1,2){ }^{*} \exp \left(\mathrm{s} 2 \mathrm{~d}^{*} t(j)\right)+$ ampt_d $(1,3$ )$* \exp (s 3 d * t(j)) ;$

$y^{3} \_t(j)=\operatorname{ampt} \_s k(1,1) * \exp (\operatorname{sisk} * t(j))$ + ampt_sk $(1,2) * \exp (s 2 s k * t(j))+$ ampt_s $\mathrm{k}(1,3) * \exp (\mathrm{s} 3 \mathrm{sk} * \mathrm{t}(\mathrm{j}))$;

$y^{4} \_t(j)=a m p t \_s k d(1,1) * \exp (\operatorname{siskd*t}(j$ )) +ampt_skd $(1,2){ }^{\star} \exp \left(\operatorname{s} 2 \operatorname{skd} d^{\star} t(j)\right)+a m$ pt_skd $(1,3) * \exp (\operatorname{s3skd*t(j));~}$ end

․ Side

zeta_sd $=(1 /(4 * p i)) * \log \left(\operatorname{amps} \_d(1,1) /\right.$ amps_d $(1,3))$;

sld=fns_d $(1,1) *\left(-z e t a \_s d+i * s q r t(1-\right.$ zeta_sd^2));

s $2 d=f n s \_d(1,2) *\left(-z e t a \_s d+i * \operatorname{sqrt}(1-\right.$ zeta_sd^2));

s3d=fns_d $(1,3) *(-$ zeta_sd+i*sqrt $(1-$ zeta_sd^2));

s4d=fns_d $(1,4) *\left(-z e t a \_s d+i * \operatorname{sqrt}(1-\right.$ zeta_sd^2)); 


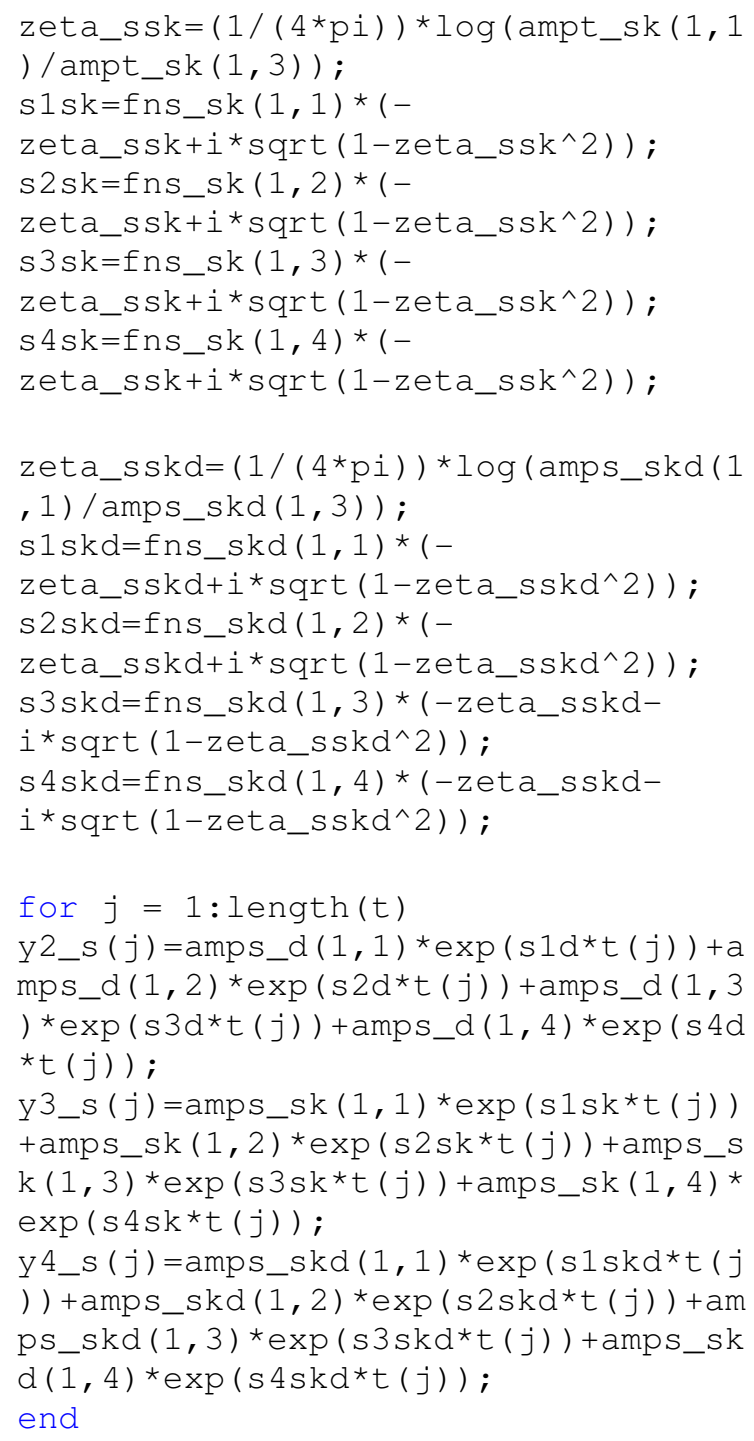

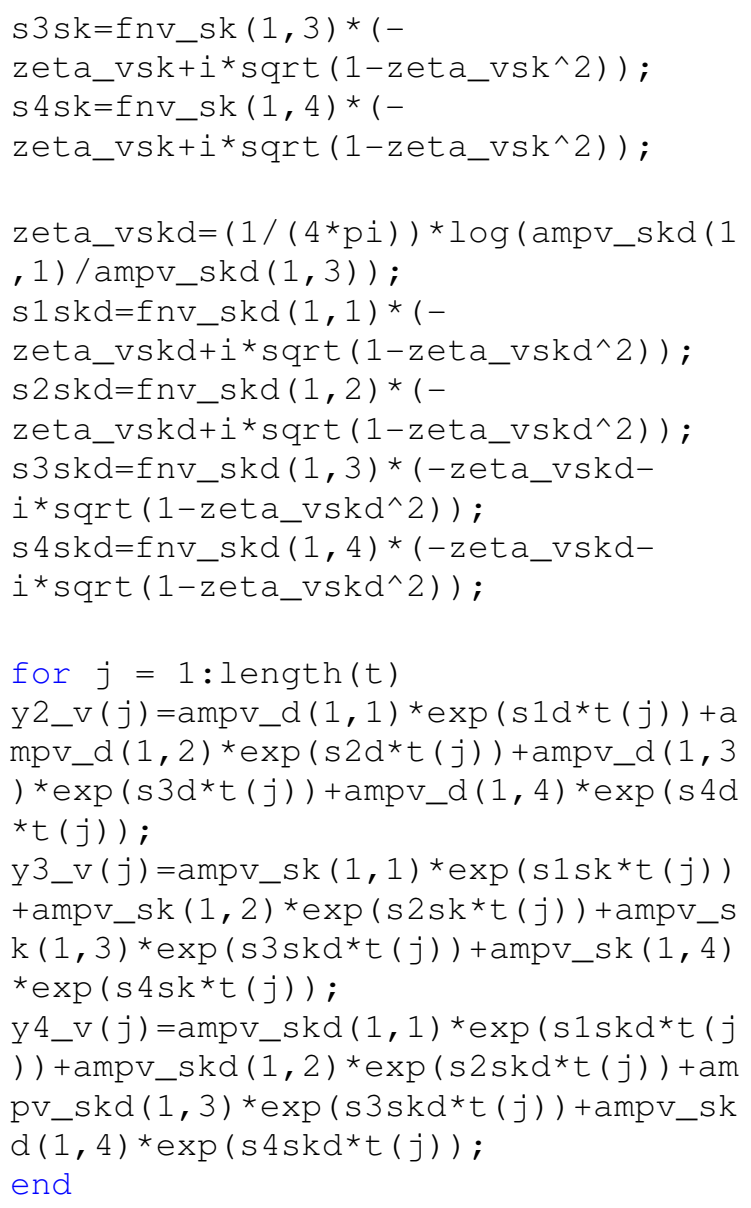

figure (12)

plot $\left(t, y, r^{\prime}, t, y^{2} \_m, ' b^{\prime}, t, y^{3} \_m,{ }^{\prime} g^{\prime}\right.$, t, y4_m, 'k')

otitle('Amplitude of the modes for the middle case')

xlabel ('Time (s)')

ylabel ('Amplitude (g) ')

legend ('Control', 'Delamination

Only', 'Shear Key Only', 'Delame \&

Shear Key')

hold on 
figure (14)

plot ( t, $y,{ }^{\prime} \mathrm{r}^{\prime}, t, \mathrm{y}^{2} \_s, \mathrm{~b}^{\prime}, t, \mathrm{y}^{3} \_s, \mathrm{~g}^{\prime}$ ' $\left.t, y^{4} \_s, k^{\prime}\right)$

otitle('Amplitude of the modes for the vertical side case')

xlabel ('Time (s)')

ylabel ('Amplitude (g) ')

legend ('Control', 'Delamination

Only', 'Shear Key Only', 'Delame \&

Shear Key')

figure (13)

plot ( t, y, 'r', t, y2_t, ' b ' , t, y3 $\mathrm{y}^{3} t, \mathrm{~g}^{\prime}$, $\left.t, y^{4} \_t,{ }^{\prime} \mathrm{k}^{\prime}\right)$

otitle('Amplitude of the modes for the top case')

xlabel ('Time (s)') ylabel ('Amplitude (g)')

legend ('Control', 'Delamination Only',' 'Shear Key Only',' 'Delame \& Shear Key')

figure (15)

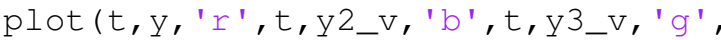
t, $\mathrm{y}^{4} \_\mathrm{v}, \mathrm{k}^{\prime}$ )

otitle('Amplitude of the modes for the vertical middle case')

xlabel('Time (s)')

ylabel ('Amplitude (g) ')

legend ('Control', 'Delamination

Only', 'Shear Key Only', 'Delame \&

Shear Key') 